\title{
MeAsurements of THE $\chi_{c}$ And $\chi_{b}$ QUARKONIUM STATES IN $p p$ COLLISIONS WITH THE ATLAS EXPERIMENT
}

\section{Andrew Stephen Chisholm}

\author{
A thesis submitted to the University of Birmingham \\ for the degree of Doctor of Philosophy
}

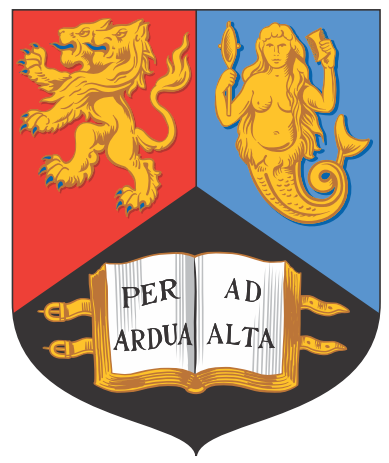

Particle Physics Group,

School of Physics and Astronomy, University of Birmingham

April 2014 



\begin{abstract}
The $\chi_{b}$ bottomonium states are observed through the reconstruction of the radiative decays $\chi_{b}(n P) \rightarrow \Upsilon(1 S) \gamma$ using $4.4 \mathrm{fb}^{-1}$ of $p p$ collision data collected at $\sqrt{s}=7 \mathrm{TeV}$ with the ATLAS experiment. The production of the $\chi_{b}(1 P)$ and $\chi_{b}(2 P)$ bottomonium states is observed in addition to a candidate for a new bottomonium state, consistent with theoretical expectations for the $\chi_{b}(3 P)$ states. The production-averaged mass barycentre for the $\chi_{b}(3 P)$ candidate is measured to be $10541 \pm 11$ (stat.) \pm 30 (syst.) $\mathrm{MeV}$. The consequences of this discovery for our understanding of bottomonium production phenomenology in hadronic collisions is reviewed.

The production of the $\chi_{c 1}$ and $\chi_{c 2}$ charmonium states has been measured in $\sqrt{s}=$ $7 \mathrm{TeV} p p$ collisions with the ATLAS experiment using a data sample representing an integrated luminosity of $4.5 \mathrm{fb}^{-1}$. The prompt and non-prompt production cross sections for the $\chi_{c 1}$ and $\chi_{c 2}$ states are measured within the region $\left|y^{J / \psi}\right|<0.75$. These measurements suggest that $24-28 \%$ of prompt $J / \psi$ are produced in feeddown from radiative $\chi_{c 1}$ and $\chi_{c 2}$ decays. The production of the $\chi_{c 2}$ state, relative to the $\chi_{c 1}$ state, is measured for both prompt and non-prompt production processes. This collection of measurements is compared to a number of theoretical predictions for $\chi_{c J}$ production at the LHC. The branching fraction $\mathcal{B}\left(B^{ \pm} \rightarrow \chi_{c 1} K^{ \pm}\right)=(4.9 \pm$ 0.9 (stat.) \pm 0.6 (syst.) $) \times 10^{-4}$ is also measured with the same dataset and $\chi_{c}$ event selection.
\end{abstract}




\section{DECLARATION OF AUTHOR'S CONTRIBUTION}

The design, construction and successful operation of the ATLAS experiment and the Large Hadron Collider are the result of a great deal of devoted work by many people. The measurements described in this thesis could not have been made without this tremendous effort. Over the course of my studies I have contributed to the operation of the ATLAS experiment by performing detector control room shifts during the summer of 2012 and conducting a number of technical studies on the ATLAS semiconductor tracker (SCT).

The measurements presented in this thesis are the result of my own work, except where explicitly stated. I was not involved in the design and construction of the ATLAS experiment and descriptions of the experiment and the relevant reconstruction algorithms in Chapters 4 and 5 are present to provide the context for the description of the measurements in Chapters 6 and 7. The analysis of the $\chi_{b}$ bottomonium states using photon conversions, described briefly in Section 6.8, is not my work and is only included to support the work described in the rest of Chapter 6. The estimates of the trigger and muon identification efficiencies described in Sections 7.5.2 and 7.5.3 are not my work but are discussed to ensure the procedure used to perform the measurements in Chapter 7 is adequately described. 


\section{ACKNOWLEDGEMENTS}

I would like to thank a number of people who have helped me, in one way or another, to prepare this thesis and the work within it. First, I thank my supervisors Chris Hawkes and Miriam Watson for the immense help and advice they have provided over the past few years and for guiding me throughout my time in Birmingham. I will always be grateful for all that you have both done for me. I would like to thank Vato Kartvelishvili, James Walder, Darren Price, Sandro Palestini and James Catmore for their considerable help and advice during the development of the measurements described in this thesis, I have learnt a great deal from all of you. I thank Pete Watkins and Paul Newman for giving me the opportunity to study in Birmingham, without your faith in me this thesis would never have been written! Thanks to the inhabitants of W316 and Bat. 104 1-B04 for providing a "stimulating" working environment; Neil Collins, Dave Hadley, Tim Martin, Jody Palmer, Tom McLaughlan, Hardeep Bansil, Benedict Allbrooke, Richard Mudd, Javier Murillo, Andrew Daniells, Mark Levy, Rhys Owen and Matt Baca. I am very grateful to the British taxpayer, through the Science \& Technology Facilities Council (STFC), for the generous financial support I have received throughout my studies. Finally, I thank my family for their love and support and thank Sarah for her love and companionship and for not complaining (too much) about sharing my attention with physics for the past few years! 


\section{Contents}

List of Figures vi vi vis

List of Tables $\quad$ xi

1 Introduction 1

2 Theoretical Foundations $\quad 3$

2.1 The Standard Model . . . . . . . . . . . . . . . . . . . . . 3

2.2 The Strong Interaction and Quantum Chromodynamics . . . . . . . . 6

3 Quarkonium 12

3.1 Quarkonium Spectroscopy . . . . . . . . . . . . . . . . 13

3.2 Quarkonium Production ................... 21

4 The ATLAS Experiment $\quad 49$

4.1 The Large Hadron Collider . . . . . . . . . . . . . . . . . . . . . . . . 49

4.2 Introduction to the ATLAS Detector . . . . . . . . . . . 52

4.3 Inner Detector . . . . . . . . . . . . . . . . . . . . 53

4.4 Calorimeter Systems _ . . . . . . . . . . . . . . . . 57

4.5 Muon System . . . . . . . . . . . . . . . . . . 6 61

4.6 Data Acquisition and Trigger System . . . . . . . . . . . . . . . . . 64

4.7 Simulation . . . . . . . . . . . . . . . . . . 67

4.8 Operation during LHC Run I . . . . . . . . . . . . . . . . . . 69 
5.1 Charged Particle (Track) Reconstruction . . . . . . . . . . . . 71

5.2 Muon Reconstruction . . . . . . . . . . . . . . . . . . 78

5.3 Photon Conversions . . . . . . . . . . . . . . . . . . . 83

5.4 Unconverted Photon Reconstruction . . . . . . . . . . . . . . . 90

5.5 Triggering of Quarkonium Decays $\mathcal{Q} \rightarrow \mu^{+} \mu^{-} \ldots \ldots$. . . . . . 91

5.6 Quarkonium Decay Reconstruction Techniques . . . . . . . . . . . . 94

5.7 Luminosity Measurement . . . . . . . . . . . . . . . . . 101

6 Observation of the $\chi_{b}$ bottomonium states 103

6.1 Introduction to the $\chi_{b}$ system . . . . . . . . . . . . 103

6.2 Reconstruction of $\chi_{b J}(n P) \rightarrow \Upsilon(m S) \gamma$ decays . . . . . . . . . 106

6.3 Observation of the $\chi_{b}$ states . . . . . . . . . . . . . . 110

6.4 Cross Checks . . . . . . . . . . . . . . . . . 114

6.5 Fitting Procedure . . . . . . . . . . . . . . . . . . . 117

6.6 Systematic Uncertainties . . . . . . . . . . . . . . . . . . . . . 121

6.7 Results . . . . . . . . . . . . . . . . . 127

6.8 Converted Photon Analysis _ . . . . . . . . . . . . . . . . . . . . . . 129

6.9 Confirmation by Other Experiments . . . . . . . . . . . . . 134

6.10 Discussion . . . . . . . . . . . . . . . . . . . . 138

6.11 Conclusion . . . . . . . . . . . . . . . . . . . . . 140

7 Measurement of the production of the $\chi_{c 1}$ and $\chi_{c 2}$ charmonium states

7.1 Introduction to the $\chi_{c}$ system . . . . . . . . . . . . . . 142

7.2 Measurement Overview . . . . . . . . . . . . . . . . . . . 146

7.3 Data and MC Simulation Samples . . . . . . . . . . . . . 147

7.4 Reconstruction of $\chi_{c J} \rightarrow J / \psi \gamma$ decays . . . . . . . . . . . 148

7.5 Cross Section Measurement Procedure . . . . . . . . . . . . . . . . . 154 
7.6 Measurement of corrected $\chi_{c J}$ yields . . . . . . . . . . . . . . . . 178

7.7 Bin Migration Corrections . . . . . . . . . . . . . . . . 188

7.8 Systematic Uncertainties . . . . . . . . . . . . . . . . . . . 193

7.9 Results and Discussion . . . . . . . . . . . . . . . . 200

7.10 Supporting Measurements . . . . . . . . . . . . . . . . . . . . 212

7.11 Conclusion . . . . . . . . . . . . . . . . . . . . 235

8 Conclusion 236

$\begin{array}{ll}\text { References } & 239\end{array}$

A Tabulated Results $\quad 252$

B Validation of $\chi_{c}$ Fitting Procedure 257 


\section{List of Figures}

3.1 The charmonium states . . . . . . . . . . . . . . . . 15

3.2 The bottomonium states . . . . . . . . . . . . . 16

3.3 Diagram of hard parton-parton scattering in a hadron-hadron collision 23

3.4 An example of a proton patron distribution function for all parton

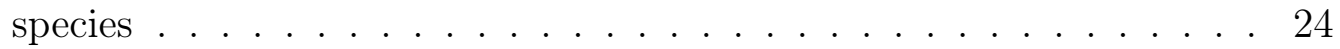

3.5 The angles relevant to the measurement of quarkonium polarisation in $V \rightarrow \ell^{+} \ell^{-}$decays. . . . . . . . . . . . . . . . . 40

3.6 CDF measurements of charmonium production in $p \bar{p}$ collisions at $\sqrt{s}=1.8 \mathrm{TeV} \ldots \ldots \ldots \ldots \ldots \ldots \ldots$

3.7 ATLAS measurements of the prompt and non-prompt $J / \psi$ production cross sections in $p p$ collisions at $\sqrt{s}=7 \mathrm{TeV} \ldots \ldots \ldots 46$

3.8 CMS measurements of the prompt $J / \psi$ and $\psi(2 S)$ production cross sections in $p p$ collisions at $\sqrt{s}=7 \mathrm{TeV} \ldots \ldots . \ldots 47$

3.9 LHCb measurement of the prompt $J / \psi$ production cross section in pp collisions at $\sqrt{s}=7 \mathrm{TeV} \ldots \ldots \ldots \ldots$. . . . . . . . 48

4.1 The layout of the $\mathrm{LHC}$ accelerator . . . . . . . . . . . . . . . 50

4.2 The layout of the CERN accelerator complex . . . . . . . . . . . 51

4.3 The ATLAS Detector . . . . . . . . . . . . . . 53

4.4 The ATLAS Inner Detector . . . . . . . . . . . . . . . . . 54

4.5 A slice through the ATLAS Inner Detector . . . . . . . . . . . . 55

4.6 The ATLAS Calorimeter Systems . . . . . . . . . . . . . . 57 
4.7 A slice through a barrel module of the ATLAS LAr EM calorimeter . 59

4.8 Segmentation of the ATLAS Hadronic Endcap calorimeter . . . . . . 60

4.9 The segmentation of the ATLAS Tile calorimeter . . . . . . . . . 61

4.10 A slice through the ATLAS muon system . . . . . . . . . . . . 63

4.11 The integrated luminosity recorded by ATLAS during 2011 and 201270

4.12 The distribution of average number of $p p$ interactions per bunch crossing measured during the 2011 and 2012 LHC runs . . . . . . . . . . 70

5.1 Definition of the ATLAS track parameters . . . . . . . . . . . . 74

5.2 Resolutions of track parameters as a function of $|\eta| \ldots . . . . .75$

5.3 Track reconstruction efficiency as a function of $p_{T}$ and $|\eta| \ldots 77$

5.4 Schematic diagram of the ATLAS muon system . . . . . . . . . 79

5.5 Efficiency of the combined muon reconstruction algorithm . . . . . 82

5.6 The total cross section for photon-atom interactions . . . . . . . . . 84

5.7 Differential cross section for photon conversion as a function of the energy sharing parameter $x \ldots \ldots \ldots$. . . . . . . . . . 85

5.8 The material budget of the ATLAS inner detector measured in radiation lengths . . . . . . . . . . . . . . 86

5.9 The photon conversion probability as a function of traversed inner detector material . . . . . . . . . . . . . . . . 87

5.10 A schematic diagram of the ATLAS muon trigger detectors . . . . . . 91

5.11 Comparison of trigger rates for ATLAS quarkonium triggers . . . . . 93

5.12 Demonstration of the resolution improvement gained using the mass difference technique in simulated $\chi_{c} \rightarrow J / \psi \gamma \rightarrow \mu^{+} \mu^{-} \gamma$ events . . . . 97

5.13 Demonstration of a simultaneous mass and decay time fit . . . . . . . 99

5.14 Definition of the impact parameter $a_{0} \ldots \ldots \ldots 10 \ldots$

6.1 Invariant mass distribution of $\Upsilon(m S) \rightarrow \mu^{+} \mu^{-}$candidates . . . . . 108 
$6.2 m\left(\mu^{+} \mu^{-} \gamma\right)-m\left(\mu^{+} \mu^{-}\right)+m_{\Upsilon(1 S)}$ distributions for $\chi_{b}(n P) \rightarrow \Upsilon(1 S) \gamma$ candidates reconstructed with unconverted photons . . . . . . . 112

$6.3 m\left(\mu^{+} \mu^{-} \gamma\right)-m\left(\mu^{+} \mu^{-}\right)+m_{\Upsilon(2 S)}$ distribution for $\chi_{b}(n P) \rightarrow \Upsilon(2 S) \gamma$ candidates reconstructed with unconverted photons . . . . . . . . 113

6.4 The mass difference $m\left(\mu^{+} \mu^{-} \gamma\right)-m\left(\mu^{+} \mu^{-}\right)+m_{\Upsilon(1 S)}$ distribution for a sample of mixed data events . . . . . . . . . . . . . . . 116

$6.5 \Delta R(\mu, \gamma)$ as a function of $m\left(\mu^{+} \mu^{-} \gamma\right)-m\left(\mu^{+} \mu^{-}\right)+m_{\Upsilon(1 S)}$ distribution for selected $\chi_{b}(n P) \rightarrow \Upsilon(1 S) \gamma$ candidates reconstructed with unconverted photons . . . . . . . . . . . . . 117

6.6 The mass difference $m\left(\mu^{+} \mu^{-} \gamma\right)-m\left(\mu^{+} \mu^{-}\right)+m_{\Upsilon(1 S)}$ distribution for a sample of simulated $\chi_{b}(1 P) \rightarrow \Upsilon(1 S) \gamma$ events . . . . . . . . . 118

6.7 Fits to $m\left(\mu^{+} \mu^{-} \gamma\right)-m\left(\mu^{+} \mu^{-}\right)+m_{\Upsilon(1 S)}$ distributions for $\chi_{b}(n P) \rightarrow$ $\Upsilon(1 S) \gamma$ candidates reconstructed with unconverted photons . . . . . 120

6.8 Fit to $m\left(\mu^{+} \mu^{-} \gamma\right)-m\left(\mu^{+} \mu^{-}\right)+m_{\Upsilon(1 S)}$ distribution for $\chi_{b}(n P) \rightarrow$ $\Upsilon(1 S) \gamma$ candidates with an alternative background PDF . . . . . . 125

6.9 Fit to $m\left(\mu^{+} \mu^{-} \gamma\right)-m\left(\mu^{+} \mu^{-}\right)+m_{\Upsilon(1 S)}$ distribution for $\chi_{b}(n P) \rightarrow$ $\Upsilon(1 S) \gamma$ candidates with no "loose" photon ID requirement imposed . 125

6.10 Simulation of the shape of contributions to the mass difference distribution from partially reconstructed bottomonium decays . . . . . . 126

6.11 Fit to $m\left(\mu^{+} \mu^{-} \gamma\right)-m\left(\mu^{+} \mu^{-}\right)+m_{\Upsilon(1 S)}$ and $m\left(\mu^{+} \mu^{-} \gamma\right)-m\left(\mu^{+} \mu^{-}\right)+$ $m_{\Upsilon(2 S)}$ distributions for $\chi_{b}(n P) \rightarrow \Upsilon(m S) \gamma$ candidates reconstructed with converted photons . . . . . . . . . . . . . 132

6.12 Kinematic acceptance for $\chi_{b}(n P) \rightarrow \Upsilon(m S) \gamma$ decays reconstructed with converted and unconverted photons . . . . . . . . . . 133

6.13 Confirmation of the new structure in $\Upsilon(1 S) \gamma$ decays by the D0 experiment.

6.14 Confirmation of the new structure in $\Upsilon(1 S) \gamma$ decays by the $\mathrm{LHCb}$ experiment 
7.1 Radidative decays in the charmonium system

7.2 Demonstration of the effect of an $a_{0}$ cut on simulated $\chi_{c J} \rightarrow J / \psi \gamma$

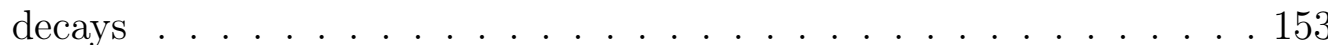

7.3 The allowed values of the $\lambda_{\theta}$ and $\lambda_{\phi}$ coefficients for $\chi_{c 1}$ and $\chi_{c 2}$ radiative decays . . . . . . . . . . . . . . . . . . . . . . 158

7.4 Acceptance maps binned in $p_{T}^{\chi_{c}}$ and $\left|y^{\chi_{c}}\right|$ for $\chi_{c 1} \rightarrow J / \psi \gamma \rightarrow \mu^{+} \mu^{-} \gamma \quad .160$

7.5 Acceptance maps binned in $p_{T}^{\chi_{c}}$ and $\left|y^{\chi_{c}}\right|$ for $\chi_{c 2} \rightarrow J / \psi \gamma \rightarrow \mu^{+} \mu^{-} \gamma \cdot 161$

7.6 Acceptance corrections binned in $p_{T}^{J / \psi}$ for $\chi_{c 1}$ and $\chi_{c 2} \ldots \ldots 2$

7.7 The single muon RoI efficiency component of the EF_2mu4 trigger efficiency, $\epsilon_{\mathrm{RoI}} \ldots \ldots \ldots \ldots \ldots$

7.8 The di-muon correction to the EF_2mu4 trigger efficiency $c_{\mu^{+} \mu^{-}} \ldots 166$

7.9 Muon identification efficiency as a function of transverse momentum and charge-signed pseudorapidity . . . . . . . . . . . . 168

7.10 The conversion probability for photons with $p_{T}^{\gamma}>1.5 \mathrm{GeV}$ as a function of $\eta^{\gamma} \ldots \ldots \ldots$. . . . . . . . . . . . . . . . . . . . . . 170

7.11 The experimental resolution in $p_{T}^{\gamma} \ldots \ldots$. . . . . . . . . 171

7.12 The effective experimental resolution in $p_{T}^{\gamma}$ including a fiducial cut on

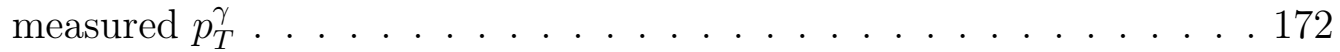

7.13 The conversion reconstruction efficiency as a function of $p_{T}^{\gamma}$ and $\left|\eta^{\gamma}\right| .173$

7.14 The conversion reconstruction efficiency as a function of $p_{T}^{\gamma}$ and $\left|\eta^{\gamma}\right| .174$

7.15 Conversion reconstruction efficiency closure tests in $p_{T}^{\chi_{c}}$ bins . . . . 176

7.16 Conversion reconstruction efficiency closure tests in $p_{T}^{J / \psi}$ bins . . . . . 177

$7.17 \Delta m$ distributions for simulated $\chi_{c J}$ decays . . . . . . . . . . . . 182

7.18 Fitted $\Delta m$ and $\tau$ distributions for the full $\chi_{c J}$ data sample . . . . . 185

7.19 Fitted $\Delta m$ and $\tau$ distributions for five $p_{T}^{J / \psi}$ bins $\ldots \ldots$. . . . . 186

7.20 Fitted $\Delta m$ and $\tau$ distributions for five $p_{T}^{\chi_{c}}$ bins $\ldots \ldots . \ldots 187$

7.21 Experimental resolution in $p_{T}^{\chi_{c}}$ and $p_{T}^{J / \psi} \ldots \ldots$. . . . . . . . . 190

7.22 Migration corrections for $p_{T}^{\chi_{c}}$ measurements . . . . . . . . . . . . . 191 
7.23 Migration corrections for $p_{T}^{J / \psi}$ measurements . . . . . . . . . . . . 192

7.24 Systematic uncertainties on the measurements of $\chi_{c 1}$ and $\chi_{c 2}$ differential cross sections . . . . . . . . . . . . . . . . . 199

7.25 Differential cross sections for prompt $\chi_{c 1}$ and $\chi_{c 2}$ measured as a func-

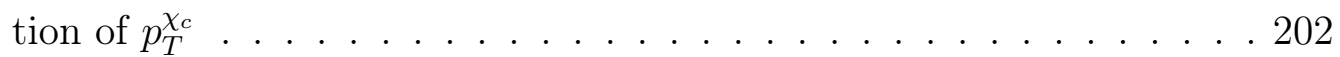

7.26 Differential cross sections for prompt $\chi_{c 1}$ and $\chi_{c 2}$ measured as a function of $p_{T}^{J / \psi} \ldots \ldots \ldots \ldots$. . . . . . . . . . . . . . . . . . . . . . . . .

7.27 Differential cross sections for non-prompt $\chi_{c 1}$ and $\chi_{c 2}$ measured as a function of $p_{T}^{\chi_{c}} \ldots \ldots \ldots \ldots \ldots$. . . . . . . . . . . . . . . . . . . . . . . .

7.28 Differential cross sections for non-prompt $\chi_{c 1}$ and $\chi_{c 2}$ measured as a function of $p_{T}^{J / \psi} \ldots \ldots \ldots$. . . . . . . . . . . . . . . . . . . . . . . . . .

7.29 The fraction of prompt $J / \psi$ produced in $\chi_{c J}$ decays, measured as a

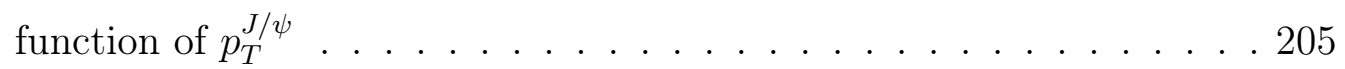

7.30 The cross section of prompt $\chi_{c 2}$ relative to prompt $\chi_{c 1}$, measured as a function of $p_{T}^{J / \psi} \ldots \ldots \ldots \ldots$. . . . . . . . . . . . . . . . . . . . . . .

7.31 The cross section of non-prompt $\chi_{c 2}$ relative to non-prompt $\chi_{c 1}$, measured as a function of $p_{T}^{J / \psi} \ldots \ldots$. . . . . . . . . . . 206

7.32 The fraction of $\chi_{c 1}$ and $\chi_{c 2}$ produced in the decays of $b$-hadrons measured as a function of $p_{T}^{\chi_{c}} \ldots \ldots \ldots$. . . . . . . . . . . 206

7.33 Comparison of $R \chi_{c}$ measurements from hadro-production experiments 209

7.34 Acceptance map for $J / \psi \rightarrow \mu^{+} \mu^{-}$decays binned in $p_{T}^{J / \psi}$ and $\left|y^{J / \psi}\right|$. . 214

7.35 Simultaneous fits to the di-muon invariant mass, $m\left(\mu^{+} \mu^{-}\right)$, and pseudoproper decay time, $\tau$, distributions of $J / \psi \rightarrow \mu^{+} \mu^{-}$candidates . . . 217

7.36 Simultaneous fits to the di-muon invariant mass, $m\left(\mu^{+} \mu^{-}\right)$, and pseudoproper decay time, $\tau$, distributions of $J / \psi \rightarrow \mu^{+} \mu^{-}$candidates . . . 218

7.37 Simultaneous fits to the di-muon invariant mass, $m\left(\mu^{+} \mu^{-}\right)$, and pseudoproper decay time, $\tau$, distributions of $J / \psi \rightarrow \mu^{+} \mu^{-}$candidates . . . 219 
7.38 The differential cross section for inclusive (prompt and non-prompt) $J / \psi$ production . . . . . . . . . . . . . . . . . 221

7.39 The differential cross sections for prompt and non-prompt $J / \psi$ pro-

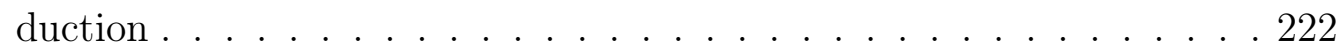

7.40 The non-prompt fraction of inclusive $J / \psi$ production . . . . . . . . 223

7.41 The $m\left(\mu^{+} \mu^{-} \gamma\right)-m\left(\mu^{+} \mu^{-}\right)$distribution of selected $B^{ \pm} \rightarrow \chi_{c 1} K^{ \pm}$ candidates . . . . . . . . . . . . . . . . 226

7.42 Fitted $B^{ \pm}$production differential cross sections . . . . . . . . . 228

7.43 Fits to the $\tilde{m}_{\chi_{c 1}}$ and $\tilde{m}_{J / \psi}$ distributions of reconstructed $B^{ \pm} \rightarrow$ $\chi_{c 1} K^{ \pm}$and $B^{ \pm} \rightarrow J / \psi K^{ \pm}$decays . . . . . . . . . . . . 231

7.44 Comparison of the measurement of $\mathcal{B}\left(B^{ \pm} \rightarrow \chi_{c 1} K^{ \pm}\right)$with existing data234

B.1 Simultaneous fits to toy MC $m\left(\mu^{+} \mu^{-} \gamma\right)-m\left(\mu^{+} \mu^{-}\right)$and $\tau$ distributions in three characteristic $p_{T}$ bins . . . . . . . . . . . . . 259

B.2 Pull distributions for prompt and non-prompt $\chi_{c 1}$ and $\chi_{c 2}$ yields in three characteristic $p_{T}$ bins . . . . . . . . . . . . . . 260 


\section{List of Tables}

2.1 Properties of the leptons . . . . . . . . . . . . . . . . . 4

2.2 Properties of the quarks . . . . . . . . . . . . . 5

2.3 Properties of the Standard Model bosons . . . . . . . . . . . . . . 5

3.1 Comparison between theoretical predictions for masses of the below threshold charmonium states and experimental values . . . . . . . . 19

3.2 Comparison between theoretical predictions for masses of the $S$ and $P$-wave bottomonium states and experimental values . . . . . . . 20

$3.3 F_{\mathcal{Q}}$ parameters for the Colour Evapouration Model . . . . . . . . 26

3.4 NRQCD matrix elements for charmonium production extracted from CDF data . . . . . . . . . . . . . . . . . . . . . 33

3.5 The mixture of $b$-hadrons measured in $Z \rightarrow b \bar{b}$ decays at LEP and $p \bar{p} \rightarrow b \bar{b}+X$ production at the Tevatron . . . . . . . . . 35

3.6 Branching fractions for the inclusive decays of $b$-hadron mixtures to final states including charmonium . . . . . . . . . . . . 36

6.1 Selected properties of the $\chi_{b J}(1 P)$ and $\chi_{b J}(2 P)$ states . . . . . . . 104

6.2 Theoretical predictions for $\chi_{b J}(3 P)$ masses . . . . . . . . . . . 105

6.3 Measured $\chi_{b}$ experimental mass barycentres . . . . . . . . . . . 128

6.4 New theoretical predictions for $\chi_{b J}(3 P)$ masses . . . . . . . . . . . . 140

7.1 Selected properties of the $\chi_{c J}(1 P)$ states $\ldots \ldots$. . . . . . . . 143

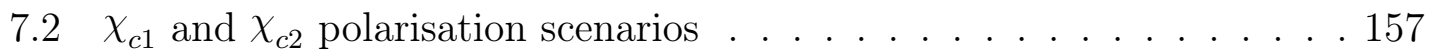


7.3 Systematic uncertainties on the cross-section measurements binned

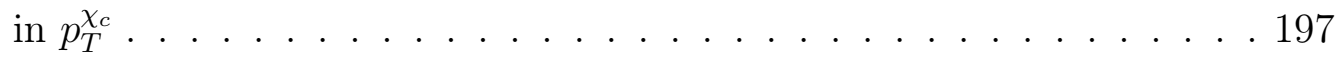

7.4 Systematic uncertainties on the cross-section measurements binned in $p_{T}^{J / \psi} \ldots \ldots \ldots \ldots \ldots$

7.5 Sources of systematic uncertainty on the measurement of $\mathcal{B}\left(B^{ \pm} \rightarrow \chi_{c 1} K^{ \pm}\right) 233$

A.1 Differential cross section for prompt $\chi_{c 1}$ and $\chi_{c 2}$ production, measured in bins of $p_{T}^{J / \psi} \ldots \ldots \ldots \ldots \ldots \ldots \ldots$. . . . . . . . . . . . . . . . . . . . . . . . . . . .

A.2 Differential cross section for non-prompt $\chi_{c 1}$ and $\chi_{c 2}$ production, measured in bins of $p_{T}^{J / \psi} \ldots \ldots \ldots \ldots$. . . . . . . . . . . . . . . . . . . . . . . . .

A.3 Differential cross section for prompt $\chi_{c 1}$ and $\chi_{c 2}$ production, measured

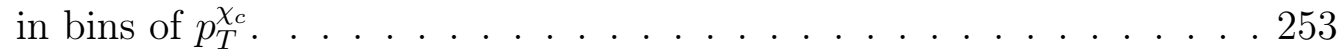

A.4 Differential cross section for non-prompt $\chi_{c 1}$ and $\chi_{c 2}$ production, measured in bins of $p_{T}^{\chi_{c}} \ldots \ldots \ldots \ldots \ldots \ldots$. . . . . . . . . . . . . . . . . . .

A.5 Fraction of prompt $J / \psi$ produced in feed-down from $\chi_{c}$ decays as a function of $p_{T}^{J / \psi} \ldots \ldots \ldots \ldots \ldots$. . . . . . . . . . . . . . . . . . . . . . . . . . . . .

A.6 Production rate of prompt $\chi_{c 2}$ relative to prompt $\chi_{c 1}$, measured in bins of $p_{T}^{J / \psi} \ldots \ldots \ldots \ldots$. . . . . . . . . . . . . . . . . . 254

A.7 Production rate of non-prompt $\chi_{c 2}$ relative to non-prompt $\chi_{c 1}$, measured in bins of $p_{T}^{J / \psi} \ldots \ldots \ldots$. . . . . . . . . . . . . . 255

A.8 Fraction of $\chi_{c 1}$ and $\chi_{c 2}$ produced in $b$-hadron decays as a function of

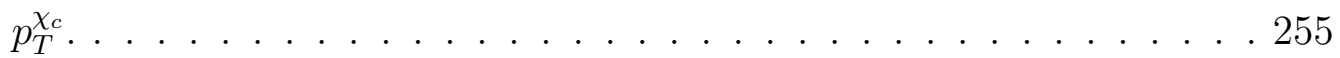

A.9 Polarisation scale factors . . . . . . . . . . . . . . . . . 256 


\section{Chapter 1}

\section{INTRODUCTION}

Nearly forty years since the discovery of the first quarkonium state, the $J / \psi[1,2]$, we cannot claim to fully understand how this quarkonium state (and the many others that were subsequently identified) is produced in hadronic interactions. Furthermore, as the experiments studying these states have become ever more sophisticated, providing increasingly more precise and diverse measurements, the situation has become even less clear and no single theoretical approach can claim to adequately describe the abundance of data that now exists. The arrival of the Large Hadron Collider (LHC), capable of delivering unprecedentedly high energy collisions and high luminosities to its cutting edge experiments, provides a unique opportunity to significantly advance our understanding of quarkonium production. This thesis describes several measurements of the production and spectroscopy of the $\chi_{c}$ and $\chi_{b}$ quarkonium states with the ATLAS experiment that can contribute to this advancement in our understanding.

Chapter 2 will briefly describe the theoretical foundations of modern particle physics that will be built upon in Chapter 3, where a theoretical and phenomenological description of quarkonium physics will be presented. The ATLAS experiment at the LHC will be discussed in Chapter 4 and a review of the experimental analysis techniques relevant to the studies presented in this thesis will be presented in Chapter 5. Chapter 6 will describe a study into the reconstruction of the $\chi_{b}$ quarkonium states with the ATLAS experiment and will describe the observation 
of a new candidate quarkonium state. Several measurements of the production of the $\chi_{c}$ quarkonium states at the ATLAS experiment will be described in Chapter 7 . Finally, the results and conclusions of this thesis will be summarised in Chapter 8 . 


\section{Chapter 2}

\section{THEORETICAL FOUNDATIONS}

\subsection{The Standard Model}

The Standard Model (SM), a modern fundamental theory of elementary particles and their interactions, represents our current understanding of the subatomic universe. The SM has enjoyed much success in describing the wealth of experimental measurements of the properties of the subatomic particles.

The SM describes all matter in the universe in terms of two classes of fundamental particles, the quarks and the leptons, with the interactions of these elementary matter particles being mediated by several gauge bosons. The SM contains six leptons and six quarks, arranged into three generations, as shown in Tables 2.1 and 2.2. The quarks and leptons are spin $\frac{1}{2}$ particles (fermions), while the particles which mediate their interactions have integer spin (bosons). The SM describes three of the four fundamental forces: electromagnetism, the strong and weak nuclear forces. In addition to the these gauge bosons, the mechanism through which the particle masses are described in the SM (the Brout, Englert, Higgs mechanism) also requires the existence of a spin zero Higgs boson. The existence of all of the standard model fermions and gauge bosons has been experimentally verified [3] and an experimental candidate for the Higgs boson has also been recently observed by the ATLAS and CMS experiments [4,5]. The properties of these bosons are summarised in Table 2.3. Gravity is not described within the SM; this is of little practical 
consequence from a phenomenological point of view but nonetheless represents an unsatisfactory situation from a theoretical perspective. The unification of all four forces within one Grand Unified Theory (GUT) is an area of active research within modern theoretical physics.

\begin{tabular}{|c|c|c|}
\hline Generation I & Generation II & Generation III \\
\hline electron & muon & tau \\
$e$ & $\mu$ & $\tau$ \\
$Q=-1$ & $Q=-1$ & $Q=-1$ \\
$m_{e}=0.51100 \mathrm{MeV}$ & $m_{\mu}=105.66 \mathrm{MeV}$ & $m_{\tau}=1776.8 \mathrm{MeV}$ \\
\hline electron neutrino & muon neutrino & tau neutrino \\
$\nu_{e}$ & $\nu_{\mu}$ & $\mu_{\tau}$ \\
$Q=0$ & $Q=0$ & $Q=0$ \\
massless $^{\dagger}$ & massless $^{\dagger}$ & massless $^{\dagger}$ \\
\hline
\end{tabular}

Table 2.1: Selected properties of the leptons. Electric charges $Q$ are presented in units of the absolute value of the electron charge. The masses of the charged leptons are taken from Ref. [3]. † In the SM, all neutrinos are described as massless particles, though recent experimental observations suggest that neutrinos have a very small mass $\ll 1 \mathrm{MeV}[3]$.

A technical discussion of the historical development of the SM, and its subsequent experimental confirmation, is beyond the scope of this thesis, though a comprehensive review can be found in Ref. [3] and the references therein. The remainder of this Chapter provides a brief description of strong interactions within the SM. 


\begin{tabular}{|c|c|c|}
\hline Generation I & Generation II & Generation III \\
\hline up & charm & top \\
$u$ & $c$ & $t$ \\
$Q=+\frac{2}{3}$ & $Q=+\frac{2}{3}$ & $Q=+\frac{2}{3}$ \\
$m_{u}=2.3_{-0.5}^{+0.7} \mathrm{MeV}$ & $m_{c}=1.275 \pm 0.025 \mathrm{GeV}$ & $m_{t}=173.07 \pm 0.52 \pm 0.72 \mathrm{GeV}$ \\
\hline down & strange & bottom \\
$d$ & $s$ & $b$ \\
$Q=-\frac{1}{3}$ & $Q=-\frac{1}{3}$ & $Q=-\frac{1}{3}$ \\
$m_{d}=4.8_{-0.3}^{+0.5} \mathrm{MeV}$ & $m_{s}=95 \pm 5 \mathrm{MeV}$ & $m_{b}=4.18 \pm 0.03 \mathrm{GeV}$ \\
\hline
\end{tabular}

Table 2.2: Selected properties of the quarks. Electric charges $Q$ are presented in units of the absolute value of the electron charge. Quark masses are taken from Ref. [3].

\begin{tabular}{|c|c|c|c|c|}
\hline Name & Mass $[\mathrm{GeV}]$ & Decay Width $[\mathrm{GeV}]$ & Spin & Charge \\
\hline$\gamma$ (photon) & 0 & - & 1 & 0 \\
$W^{ \pm}$ & $80.385 \pm 0.015$ & $2.085 \pm 0.042$ & 1 & \pm 1 \\
$Z^{0}$ & $91.1876 \pm 0.0021$ & $2.4952 \pm 0.0023$ & 1 & 0 \\
$g$ (gluon) & 0 & - & 1 & 0 \\
$H$ (Higgs) & $125.9 \pm 0.4$ & $4.15 \times 10^{-3}$ (th.) $[6]$ & 0 & 0 \\
\hline
\end{tabular}

Table 2.3: Selected properties of the SM bosons. Electric charges are presented in units of the absolute value of the electron charge. All data are taken from Ref. [3]. 


\subsection{The Strong Interaction and Quantum Chromo- dynamics}

Our modern understanding of the strong interaction began in 1963, when Gell-Mann and Zweig postulated that the strongly interacting particles observed by experiments (hadrons such as the proton, neutron and pion) are not fundamental particles, but instead bound states composed of more basic constituents [7]. These constituents, which are now thought to be fundamental particles, subsequently became known as the quarks. Even at the time of the proposal, the properties of the known spectrum of hadrons put tight constraints on the properties of the quarks. The $\pi$ and $\rho$ mesons were known to have $J^{P}=0^{-}$and $1^{-}$respectively (where $J$ and $P$ denote the total angular momentum and parity quantum numbers, respectively). These mesons could naturally be interpreted as bound states of a quark and an anti-quark with zero orbital angular momentum. The known hadrons could be classified within an $S U(3)$ group of three spin $1 / 2$ particles, each with a unique flavour; the up $(u)$, down $(d)$ and strange $(s)$ quarks. For the model to also predict the baryon spectrum, the quarks were constrained to be fractionally charged $(+2 / 3 e$ for $u$ and $-1 / 3 e$ for $d$ and $s$ quarks). At first glance, this model naturally explained the known spectra of mesons and baryons, but one important inconsistency suggested that the model was not yet complete.

This model could not explain the existence of baryons consisting of three quarks of the same flavour with total angular momentum $J=3 / 2\left(\right.$ e.g. $\Delta^{++}$), despite the firm experimental evidence. Quarks were thought to be spin 1/2 particles and so Fermi-Dirac statistics required that the overall baryon wavefunction be totally anti-symmetric under the exchange of any pair of quarks. To reconcile this issue, a further quantum number was introduced, intrinsic only to quarks; colour. If the baryon wavefunction were totally symmetric under the exchange of spin and flavour then requiring that the wavefunction be anti-symmetric under the exchange of colour 
quantum numbers would allow the overall wavefunction to be totally anti-symmetric, consistent with the expectation for fermions [7]. While the quark model was phenomenologically successful, and the two most important concepts in our modern understanding of the strong interaction, quarks and colour, had been introduced, the model raised just as many questions as it answered. For example, the new colour degrees of freedom could lead to a spectrum of hadronic states far richer than that observed at the time. This led to the ad-hoc requirement that only colour singlet states can exist in nature. However, it would not be until the development of Quantum Chromodynamics (QCD) that a satisfactory explanation of this feature could be provided, along with answers to many of the other remaining questions.

Quantum Chromodynamics (QCD) is a non-abelian gauge theory based upon an $S U(3)$ gauge group [8]. To some extent QCD is an analogue of QED, in that it is a theory of fundamental fermions (quarks) that possess a "charge", known as colour. The gauge boson of QCD, the particle that mediates all interactions, is known as the gluon, which is the analogue of the photon of QED. However, QED and QCD differ in one important aspect that leads to some very different qualitative properties. The gluon itself has a colour "charge" (unlike the electrically neutral photon), a feature inherited from the non-abelian nature of QCD (the elements of the $S U(3)$ gauge group do not commute). It is this feature of the theory that leads to most of the important phenomenological aspects of QCD and provides the bridge that enables QCD to be a self-consistent theory of both quarks and hadrons.

The Lagrangian density for QCD can be written as

$$
\mathcal{L}_{Q C D}=-\frac{1}{4} F_{a}^{\mu \nu} F_{\mu \nu}^{a}+\sum_{j=1}^{N_{f}} \bar{\psi}_{j}^{\alpha}\left(i \gamma_{\mu} D^{\mu}-m_{j}\right)_{\alpha \beta} \psi_{j}^{\beta}-\frac{1}{2 \lambda}\left(\partial^{\mu} A_{\mu}^{a}\right)^{2}-\partial_{\mu} \bar{\phi}_{a} D^{\mu} \phi^{a}
$$

The first two terms describe the interaction of $N_{f}$ flavours of spin $1 / 2$ quarks $\psi_{j}$ (Dirac spinors with a colour field) of masses $m_{j}$ with massless spin 1 gluons [8]. 
The $\gamma^{\mu}$ represent the Dirac gamma matrices. The quantity $\left(D^{\mu}\right)_{\alpha \beta}$ is the covariant derivative and is given by

$$
\left(D^{\mu}\right)_{\alpha \beta}=\delta_{\alpha \beta} \partial^{\mu}-\frac{i}{2} g \sum_{a} \lambda_{\alpha \beta}^{a} A^{a \mu}
$$

where $g$ is the coupling constant and the indices $\alpha$ and $\beta$ run over the three quark colours. The $\lambda_{\alpha \beta}^{a}$ are the eight $3 \times 3$ Gell-Mann colour matrices (Hermitian and traceless) [9]. $F_{\mu \nu}^{a}$ is the QCD field strength tensor and is given by

$$
F_{\mu \nu}^{a}=\partial_{\mu} A_{\nu}^{a}-\partial_{\nu} A_{\mu}^{a}-g f^{a b c} A_{\mu}^{b} A_{\nu}^{c}
$$

where $A_{\mu}^{a}$ is the gluon field and the indices $a, b$ and $c$ run over the octet of 8 gluon colours [8]. The third term in Equation 2.3 is absent in the analogous QED field strength tensor and gives rise to gluon self-interactions. The $f^{a b c}$ are the structure constants of the QCD $S U(3)$ colour group. The generators of the $S U(3)$ group satisfy

$$
\left[T^{a}, T^{b}\right]=i f^{a b c} T^{c}
$$

where $\left(T^{a}\right)_{\alpha \beta}=\frac{1}{2} \lambda_{\alpha \beta}^{a}$ in the colour triplet fundamental representation and $\left(T^{a}\right)_{b c}=$ $-i f^{a b c}$ in the adjoint colour octet gluon representation [9]. The third term in the QCD Lagrangian density shown in Equation 2.1 is the gauge fixing term, which allows perturbative calculations to be made. It allow a allows a gluon propagator to be defined by making a choice of gauge through setting the parameter $\lambda$ (typical choices include the Feynman gauge $\lambda=1$ and Landau gauge $\lambda=0$ ) [8]. The final term describes the Faddeev-Popov ghost fields $\phi$, which cancel degrees of freedom that lead to unphysical particles [8].

The QCD Lagrangian possesses several important symmetry properties, which lead to some interesting and necessary characteristics of the theory. The physical properties of the QCD Lagrangian density are unchanged if the quark and gluon 
fields are redefined, and it is thus locally gauge invariant. It is this property (common also to the other components of the SM) that allows QCD to be a renormalisable quantum field theory that can be used to perform calculations [8]. The strong interaction has been experimentally verified also to be invariant under several discrete transformations, including charge conjugation $C$, parity $P$ and time reversal $T$. However, some terms can be added to the QCD Lagrangian which would violate the symmetry under both $P$ and $T$ transformations, despite the stringent experimental constraints. This is a still unresolved phenomenon known as the strong CP problem. In addition to these exact symmetries, QCD also possesses several approximate symmetries that lead to some other interesting properties. In the limit that the masses of the light $(u, d, s)$ quarks are degenerate, QCD naturally leads to isospin $I$ symmetry (i.e. symmetry under the interchange of $u$ and $d$ quarks) and gives rise to the $S U(3)$ baryon and meson multiplets of Gell-Mann's eightfold way [8]. In the limit that the light quarks are massless, a further chiral symmetry emerges which can be exploited to perform perturbative calculations at energy scales close to the hadronic scale [8].

The calculation of physical observables in QCD usually results in a perturbative series in powers of the strong coupling $\alpha_{\mathrm{S}}=g^{2} / 4 \pi$. This calculational process involves a renormalisation procedure, which removes the divergences that are often present in the perturbative series to allow a finite physical result to be obtained. However, this renormalisation procedure has to be performed at an arbitrary mass scale $\mu$. This leads to a dependence of the calculated physical observable on the arbitrary renormalisation scale $\mu$ in addition to any intrinsic energy scale $Q$. However, physically observable quantities cannot have a dependence on arbitrary scales. This problem is solved by requiring that any dependence on $\mu$ is contained only within $\alpha_{\mathrm{S}}$ (since dimensionless physical observables can depend only on the ratio $Q / \mu$ ). If one further requires that the calculation of a physical observable is independent of $\mu$, then a running strong coupling, with an explicit dependence on $Q$, can be used 
to absorb this dependence on $\mu$;

$$
\alpha_{\mathrm{S}}(Q)=\frac{\alpha_{\mathrm{S}}}{1+\left(b_{0} \alpha_{\mathrm{S}} / 2 \pi\right) \ln (Q / \mu)}
$$

The coefficient $b_{0}$ is the leading order coefficient in the QCD $\beta$-function, $b_{0}=11-$ $2 N_{f} / 3$ for QCD with three colours [7]. Now that all the dependence on $\mu$ has been absorbed into $\alpha_{\mathrm{S}}$, it can be removed entirely by defining a mass-scale cut-off known as the QCD scale, $\Lambda_{\mathrm{QCD}}$, which satisfies $\left(\alpha_{\mathrm{S}} b_{0} / 2 \pi\right) \ln \left(\mu / \Lambda_{\mathrm{QCD}}\right)=1$ (the scale at which the coupling diverges). This can be substituted into Equation 2.5 to give

$$
\alpha_{\mathrm{S}}(Q)=\frac{2 \pi}{b_{0} \ln \left(Q / \Lambda_{\mathrm{QCD}}\right)}
$$

This equation shows that as $Q$ increases, the coupling decreases. The reduction of $\alpha_{\mathrm{S}}$ as $Q$ increases is known as asymptotic freedom and provides the basis upon which perturbative calculations can be performed within QCD. Experimental measurements suggest that $\Lambda_{\mathrm{QCD}} \approx 200 \mathrm{MeV}$. Thus for $\alpha_{\mathrm{S}}$ to be small enough to remain a good perturbative expansion parameter (e.g. less than around 0.4), the energy scale should be sufficiently high, $Q>1 \mathrm{GeV}$. Thus, $\Lambda_{\mathrm{QCD}}$ sets the scale at which QCD moves from being a perturbative theory of asymptotically free quarks to a non-pertubative theory.

At energy scales near $\Lambda_{\mathrm{QCD}}$, where the coupling is strong, quarks are always confined within hadrons. The apparently patternless hierarchy of the quark masses leads to a rich spectrum of hadrons that exhibit many interesting properties. The theoretical description of the structure of hadrons is typically semi-empirical though non-perturbative calculation methods such as Lattice QCD can be used to describe the behaviour of hadrons directly in terms of the QCD degrees of freedom. Despite the theoretical limitations, hadronic physics can provide a unique window on many aspects of the strong interaction. In particular, the theoretical description of the bound states of heavy quarks is often less complicated by non-perturbative dynam- 
ics. The theoretical and experimental study of such bound states has led to many advances in our understanding of the strong interaction and remains an important area of research, as described in the following Chapter. 


\section{Chapter 3}

\section{QUARKONIUM}

Quarkonium is the name given to a sub-atomic system composed of a heavy quark $Q$ and anti-quark $\bar{Q}$, bound by the strong interaction. Quarkonia belong to the more general meson (hadrons composed of a quark anti-quark pair) family of subatomic particles but they deserve their own sub-classification due to the many unique properties that distinguish them from the other hadrons. The large masses of the charm $(c)$ and bottom $(b)$ quarks compared to the light $(u, d, s)$ quarks, endow heavy quarkonia with properties that differ significantly from those of the light mesons. A quarkonium system containing a charm and anti-charm $(c \bar{c})$ quark pair is known as charmonium, while the system containing a bottom and anti-bottom $(b \bar{b})$ quark pair is known as bottomonium. Charmonium and bottomonium have both been observed in experiments [3]. The large mass of the top quark affords it with a width so large that it decays via the weak interaction on a timescale below that associated with quarkonium formation. To date, quarkonia composed of top antitop $(t \bar{t})$ quark pairs (an hypothesised system known as toponium) have not been observed experimentally [3].

Charmonium was first observed experimentally in November 1974 with the famous simultaneous discovery of a narrow state decaying to $e^{+} e^{-}$and $\mu^{+} \mu^{-}$by groups at BNL and SLAC $[1,2]$. The new state had a mass of around $3.1 \mathrm{GeV}$ and was quickly interpreted as a bound state of a previously unseen heavy quark and its anti-quark, much heavier than the strange quark. The new quark was named the 
charm (c) quark and the new bound state came to be known as the $J / \psi$. Only a few years later in 1977, a further narrow state was observed in decays to $\mu^{+} \mu^{-}$by a group at Fermilab, this time at a mass of around 9.5 GeV [10]. This new state was named $\Upsilon$ (now known as $\Upsilon(1 S)$ ) and provided the first evidence for a further heavy quark, the bottom $(b)$ quark. Following the discovery of the $J / \psi$ and $\Upsilon(1 S)$ states, many other quarkonium states were discovered, the masses and quantum numbers of which fitted well with theoretical expectations for $Q \bar{Q}$ bound states.

\subsection{Quarkonium Spectroscopy}

Quarkonium states are typically categorised according to the total spin of the $Q \bar{Q}$ system $S$, the orbital angular momentum between the $Q \bar{Q}$ pair, $L$, and the total angular momentum $(\vec{J}=\vec{L}+\vec{S})$ of the system, $J$. The spectroscpic notation $n^{2 S+1} L_{J}$, where $n$ is the principal quantum number, is often used to label the quarkonium states. The parity $P$ of a $Q \bar{Q}$ system is given by $P=(-1)^{L+1}$ and the charge conjugation parity $C$ is given by $C=(-1)^{L+S}$. Parity and charge conjugation parity are both conserved quantities in the strong and electromagnetic decays of quarkonium states.

The quarkonium states with masses below the threshold for decays to openflavour hadrons (i.e. containing non-zero flavour quantum numbers) to be kinematically allowed $\left(m_{D \bar{D}} \approx 3.74 \mathrm{GeV}\right.$ for charmonium and $m_{B \bar{B}} \approx 10.56 \mathrm{GeV}$ for bottomonium) are typically narrow states which decay via the electromagnetic and strong interactions to lower mass quarkonium states, light hadrons or charged leptons. Quarkonium states above the relevant open-flavour threshold generally have much larger total decay widths that are dominated by strong decays to open-flavour hadrons. All of the charmonium states expected to exist below the $D \bar{D}$ threshold have been discovered experimentally. The majority of the bottomonium states expected below the $B \bar{B}$ threshold have also been observed with the exception of the 
$\eta_{b}(3 S), h_{b}(3 P)$ and most of the $D$-wave bottomonium states [3]. Figures 3.1 and 3.2 show the experimentally observed (and predicted) charmonium and bottomonium states respectively.

The properties of the experimentally observed quarkonium states are known very well in some cases while very little is known about others. In general, the $S$-wave $(L=0)$ vector $(S=1) J^{P C}=1^{--}$states, such as the $J / \psi$ and $\Upsilon$, are very well studied since they can be produced directly in $e^{+} e^{-}$annihilation and can thus decay to the experimentally clean $e^{+} e^{-}$and $\mu^{+} \mu^{-}$final states. The $P$-wave $(L=1)$ spin triplet $(S=1) \chi$ states are also reasonably well studied as they are readily produced in the radiative decays of the vector states and can decay to lower lying vector states with the emission of a photon. However, much less is known about the spin singlet $(S=0) S$-wave states, $\eta_{c}$ and $\eta_{b}$, the latter of which $\left(\eta_{b}(1 S)\right)$ was only recently discovered in 2008 [11]. The di-lepton decays of these states are heavily suppressed, with decays to light hadrons dominating. These states are typically studied through radiative decays of the type ${ }^{3} S_{1} \rightarrow{ }^{1} S_{0} \gamma$, although this radiative transition is dominated by a magnetic dipole amplitude and is strongly suppressed relative to the analogous electric dipole transition. Less still is known about the spin singlet $P$-wave states, $h_{c}$ and $h_{b}$, the decays of which are also dominantly hadronic.

The quarkonium spectrum can be understood from a theoretical perspective through two distinct approaches. The first approach is largely phenomenological and involves the use of potential models to describe the bound state, in analogy with the quantum mechanical description of the energy levels of an atom. The potential model approach is attractive because of its simplicity, but becomes steadily more complicated and inaccurate for the heavier quarkonium states (particularly those above the open flavour thresholds). The alternative approach is to perform a direct calculation using QCD. Such calculations can be performed with lattice QCD but are complex and (very) computationally expensive; few lattice calculations exist relative to the many potential model calculations in the literature. However, lattice 


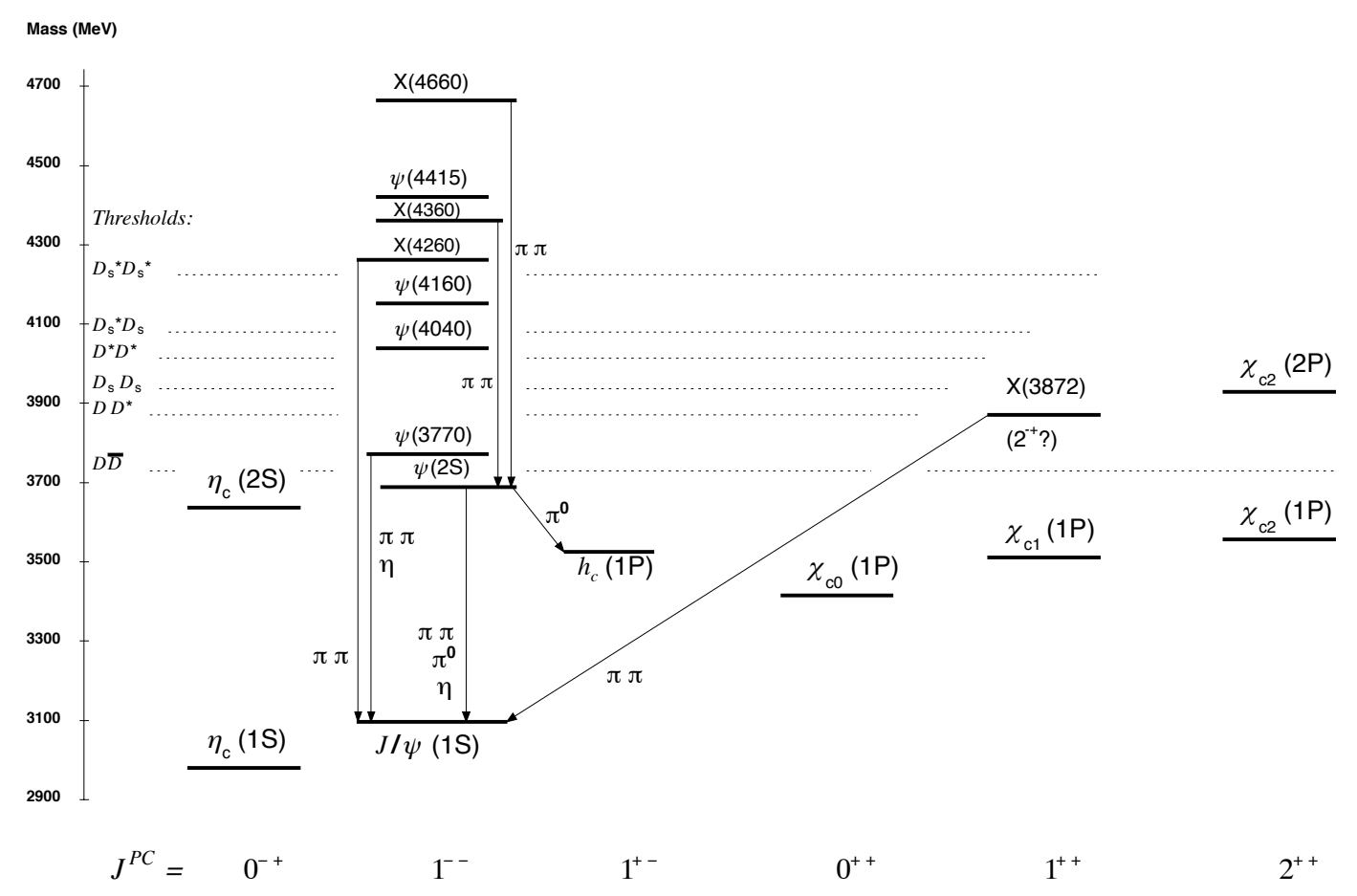

Figure 3.1: The experimentally observed charmonium states. The states labelled $X$, the nature of which is unknown, are not thought to be conventional charmonium states. Figure from Ref. [3].

calculations that use the effective theory Non-Relativistic QCD (NRQCD) have successfully described much of the charmonium and bottomonium spectrum [13-15].

The main features of the experimentally observed spectrum of quarkonium states can be reproduced with a simple non-relativistic potential model. The use of an interaction potential and "ordinary" non-relativistic quantum mechanics to model the system is well motivated given the large masses of the charm and bottom quarks relative to the QCD scale, $\Lambda_{\mathrm{QCD}}$. The potential $V(\vec{r})$ can be parameterised as a function of the relative separation, $\vec{r}$, between the quark $Q$ and the anti-quark $\bar{Q}$. The problem is simplified mathematically if one models the system as an anti-quark orbited by a quark with mass $\mu$ bound by an attractive relative central potential $V(r)$ (a common technique used in the textbook [16] solution of the Schrödinger equation for the hydrogen atom). The parameter $\mu$ is known as the reduced mass. As the mass of the heavy quark and anti-quark are equal, the reduced mass is simply $\mu=m_{Q} / 2$, where $m_{Q}$ is the mass of the heavy quark. If the spin of the quarks 


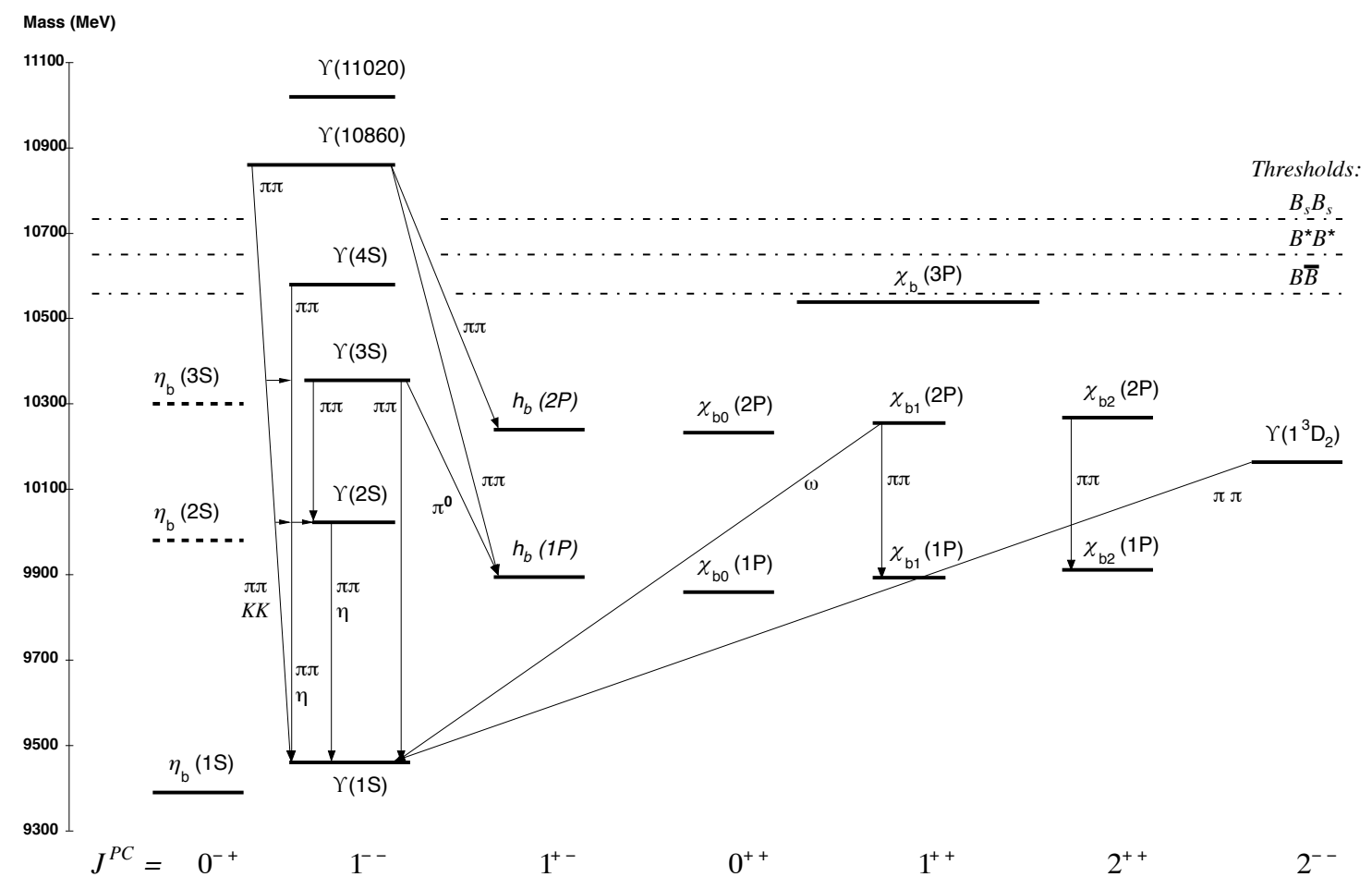

Figure 3.2: The experimentally observed and theoretically expected bottomonium states. Dashed lines denote unobserved or unconfirmed states (an unconfirmed experimental candidate for the $\eta_{b}(2 S)$ state has been observed by the Belle experiment [12]). Figure from Ref. [3].

is neglected, the binding energy $E_{n l}$ of the bound state can be found by solving the time-independent Schrödinger equation (in natural units with $\hbar=1$ ) for the potential,

$$
\left[-\frac{\nabla^{2}}{2 \mu}+V(r)\right] \psi_{n l m}(r, \theta, \phi)=E_{n l} \psi_{n l m}(r, \theta, \phi)
$$

where $\theta$ and $\phi$ are the polar and azimuthal co-ordinates of the heavy quark [16]. The quantum numbers $n, l$ and $m$ are the principal quantum number, orbital angular momentum quantum number and its projection onto the $z$-axis respectively. The mass of the quarkonium state is then given by $M_{n l}=E_{n l}+2 m_{Q}$. The parametrisation of the problem with a spherically symmetric central potential in polar coordinates allows the wavefunction $\psi_{n l m}(r, \theta, \phi)$ to be factorised into a product of three functions, $\psi_{n l m}(r, \theta, \phi)=R_{n l}(r) \Theta_{l m}(\theta) \Phi_{m}(\phi)$. The product $\Theta_{l m}(\theta) \Phi_{m}(\phi)$ is described by the 
spherical harmonic functions $Y_{l m}(\theta, \phi)$ [16]. Thus the total wavefunction is given by $\psi_{n l m}(r, \theta, \phi)=R_{n l}(r) Y_{l m}(\theta, \phi)$. The parametrisation of the solution can be simplified further with the introduction of the reduced radial wavefunction $u_{n l}(r)=r R_{n l}(r)$. The Schrödinger equation can then be written as,

$$
\frac{d^{2} u_{n l}(r)}{d r^{2}}=\frac{1}{2 \mu}\left[E_{n l}-V(r)-\frac{l(l+1)}{2 \mu r^{2}}\right] u_{n l}(r)
$$

The boundary conditions $u_{n l}(0)=0$ and $u_{n l}^{\prime}(0)=R_{n l}(0)$ must also be imposed to remove unphysical solutions [9]. Finally, the normalisation condition,

$$
\int|\psi|^{2} r^{2} \sin \theta d \theta d \phi d r=\int_{0}^{\infty}\left|u_{n l}(r)\right|^{2} d r=1
$$

must also be imposed to preserve unitarity [9].

The form of the potential can be predicted from the qualitative features of QCD. At short distances, less than $1 \mathrm{fm}$, corresponding to energies greater than the QCD scale, $\Lambda_{\mathrm{QCD}}$, the potential can be calculated with perturbative QCD. The QCD static potential can be derived from the leading order contribution (single gluon exchange) to the $Q \bar{Q} \rightarrow Q \bar{Q}$ scattering amplitude. This leads to a potential with a form analogous to the Coulomb potential of QED,

$$
V(r)=-\mathcal{C} \frac{\alpha_{\mathrm{S}}}{r}
$$

where $\alpha_{\mathrm{S}}$ is the strong coupling constant and $\mathcal{C}$ is a constant factor related to the colour configuration of the $Q \bar{Q}$ state. For a $Q \bar{Q}$ pair in a colour singlet configuration $\mathcal{C}=4 / 3$, while for a colour octet configuration $\mathcal{C}=-1 / 6$. It is important to note that the overall potential is attractive $(V<0)$ only for the colour singlet configuration, and so $Q \bar{Q}$ pairs in a colour octet configuration can not form bound states, a feature consistent with experimental observations.

At distances beyond around $1 \mathrm{fm}$, corresponding to energies similar to or less than $\Lambda_{\mathrm{QCD}}$, the coupling becomes strong, leading to a confinement regime. In this 
regime, perturbative QCD is no longer valid as $\alpha_{s}$ is no longer small. Instead, a phenomenologically motivated potential must be used. The most common model is still inspired by the qualitative features of QCD, where a colour field flux tube forms between colour charges separated by large distances, leading to a distance independent confining force [17]. This can be interpreted as a long range linear confining potential,

$$
V(r)=K r
$$

where $K$ is a parameter often called the QCD string tension [9]. The parameter $K$ is chosen with input from the experimentally measured spectrum and typically takes values of around $0.18 \mathrm{GeV}^{2}$ [17]. This "Coulomb + Linear" potential (alternative parametrisations also exist, such as the Cornell [18] and Richardson [19] potentials) can be used to provide a reasonably good description of the spin-independent features of the experimentally measured charmonium and bottomonium spectra. The QCD static potential can also be calculated to higher perturbative orders to provide a more accurate description which includes an explicit running of the strong coupling. This naturally leads to a confining behaviour at large separations, negating the need for the phenomenologically motivated additional terms [9]. In addition to higher order QCD corrections, corrections to the non-relativistic approximation can also be added to improve the accuracy of the model. Further spin-dependent terms must be included to reproduce the hyperfine splitting between the ${ }^{1} S_{0}\left(\eta_{c}, \eta_{b}\right)$ and ${ }^{3} S_{1}(J / \psi, \Upsilon)$ states and the fine structure of the $P$-wave $\chi$ states. The addition of terms that model the spin-orbit and hyperfine interactions leads to much more precise predictions that can be directly compared with experimental measurements.

Tables 3.1 and 3.2 show a collection of potential model and lattice QCD predictions for the masses of selected charmonium and bottomonium states. The agreement of modern potential model predictions with the experimental values is very good [20]. Recent lattice QCD results are also in reasonable agreement with data 
but tend to have larger theoretical uncertainties [13-15].

\begin{tabular}{|c|c|c|c|c|c|}
\hline Expt. State & $n^{2 S+1} L_{J}$ & Expt. Mass & Pot. [21] & Pot. [20] & Latt. [13] \\
\hline$\eta_{c}(1 S)$ & $1^{1} S_{0}$ & $2983.7(7)$ & 2975 & 2979 & $3000(2)$ \\
$J / \psi$ & $1^{3} S_{1}$ & $3096.92(1)$ & 3098 & 3096 & Fixed to expt. \\
\hline$h_{c}$ & $1^{1} P_{1}$ & $3525.4(1)$ & 3517 & 3526 & - \\
$\chi_{c 0}$ & $1^{3} P_{0}$ & $3414.8(3)$ & 3445 & 3424 & - \\
$\chi_{c 1}$ & $1^{3} P_{1}$ & $3510.66(7)$ & 3510 & 3510 & - \\
$\chi_{c 2}$ & $1^{3} P_{2}$ & $3556.20(9)$ & 3550 & 3556 & - \\
\hline$\eta_{c}(2 S)$ & $2^{1} S_{0}$ & $3639.4(1)$ & 3623 & 3588 & $3680(6)$ \\
$\psi(2 S)$ & $2^{3} S_{1}$ & $3686.109(1)$ & 3676 & 3686 & $3717(8)$ \\
\hline
\end{tabular}

Table 3.1: Theoretical predictions for the masses of the charmonium states below the $D \bar{D}$ threshold. Predictions from potential models (Pot.) [20,21] and lattice QCD (Latt.) [13] are compared to the world average experimental masses [3]. All masses are quoted in units of $\mathrm{MeV}$.

Within the past ten years, many new states have been observed which decay to quarkonium. These new states, typically denoted by $X, Y$ or $Z$, have properties that do not obviously fit into the conventional quarkonium model. These states have become known as quarkonium-like states and their nature is still far from well understood. The first such state to be discovered was the $X(3872)$, observed by the Belle experiment in $B^{ \pm}$meson decays to $J / \psi \pi^{+} \pi^{-} K^{ \pm}$final states (with $\left.X(3872) \rightarrow J / \psi \pi^{+} \pi^{-}\right)[22]$. The discovery was promptly confirmed by CDF [23] and D0 [24] (in $p \bar{p}$ collisions, through predominantly prompt production) with several other experiments (including the LHC experiments) subsequently confirming the state's existence. The $X(3872)$ is observed to be a narrow state (with an experimental width consistent with detector resolution) but has a mass above the $D \bar{D}$ threshold. The observation of the $X(3872)$ came as a shock since all known charmonium states with masses above the $D \bar{D}$ threshold are broad states that readily decay to open-charm hadrons. Many theoretical interpretations of the $X(3872)$ have been proposed, including hadronic molecules, tetra-quark states and hybrid charmonium, though its nature is still not firmly established [25]. Since the discovery of the $X(3872)$, many other charmonium-like and bottomonium-like states have also been 


\begin{tabular}{|c|c|c|c|c|c|}
\hline Expt. State & $n^{2 S+1} L_{J}$ & Expt. Mass & Pot. [21] & Pot. [20] & Latt. [14] \\
\hline$\eta_{b}(1 S)$ & $1^{1} S_{0}$ & $9398(3)$ & 9402 & 9400 & $9390(9)$ \\
$\Upsilon(1 S)$ & $1^{3} S_{1}$ & $9460.3(3)$ & 9465 & 9460 & - \\
\hline$h_{b}(1 P)$ & $1^{1} P_{1}$ & $9899(1)$ & 9882 & 9901 & $9905(7)$ \\
$\chi_{b 0}(1 P)$ & $1^{3} P_{0}$ & $9859.4(4)$ & 9847 & 9865 & - \\
$\chi_{b 1}(1 P)$ & $1^{3} P_{1}$ & $9892.8(3)$ & 9876 & 9892 & - \\
$\chi_{b 2}(1 P)$ & $1^{3} P_{2}$ & $9912.2(3)$ & 9897 & 9913 & - \\
\hline$\eta_{b}(2 S)$ & $2^{1} S_{0}$ & $9999(4)$ & 9976 & 9993 & $9988(3)$ \\
$\Upsilon(2 S)$ & $2^{3} S_{1}$ & $10023.3(3)$ & 10003 & 10023 & - \\
\hline$h_{b}(2 P)$ & $2^{1} P_{1}$ & $10259.8(1)$ & 10250 & 10261 & - \\
$\chi_{b 0}(2 P)$ & $2^{3} P_{0}$ & $10232.5(5)$ & 10226 & 10234 & - \\
$\chi_{b 1}(2 P)$ & $2^{3} P_{1}$ & $10255.5(5)$ & 10246 & 10255 & - \\
$\chi_{b 2}(2 P)$ & $2^{3} P_{2}$ & $10268.7(5)$ & 10261 & 10268 & - \\
\hline$\eta_{b}(3 S)$ & $3^{1} S_{0}$ & - & 10336 & 10328 & - \\
$\Upsilon(3 S)$ & $3^{3} S_{1}$ & $10355.2(5)$ & 10354 & 10355 & $10375(22)$ \\
\hline
\end{tabular}

Table 3.2: Theoretical predictions for the masses of the $S$ and $P$-wave bottomonium states. Predictions from potential models (Pot.) [20,21] and lattice QCD (Latt.) [14] are compared to the world average experimental masses [3]. All masses are quoted in units of $\mathrm{MeV}$.

observed (including manifestly exotic charged states), in multiple final states with varying levels of confirmation $[3,25]$. These observations have prompted a significant amount of recent theoretical work. However, to date, no single theoretical interpretation can claim to describe the full spectrum of new states adequately, suggesting that multiple mechanisms may be at play. The LHC experiments and $B$ factories are actively studying these states and new measurements of production cross sections and quantum numbers are beginning to shed more light on these mysterious states $[26,27]$. 


\subsection{Quarkonium Production}

Quarkonium states can be produced in a variety of different interactions at modern experiments. Common initial states include hadron-hadron collisions (typically $p p$ or $p \bar{p}$ at collider experiments), hadron-nucleon (typically $p$ or $\bar{p}$ on a fixed nuclear target), $e^{+} e^{-}$collisions, $\gamma \gamma$ collisions and ep collisions. The following discussion will focus on the production of quarkonium states in hadronic collisions. Various models describing the production of quarkonium in hadronic collisions exist. The following discussion will attempt to summarise the main models with more emphasis given to the more recent and successful ones.

\subsubsection{The QCD factorisation method}

Particle production in hadronic collisions can be described within QCD in terms of the interactions between the constituent quarks and gluons (also known as partons) within the colliding hadrons. The cross section for the process $H_{1}\left(P_{1}\right)+H_{2}\left(P_{2}\right) \rightarrow X$, where $H_{1,2}$ are initial state hadrons with four-momentum $P_{1,2}$ and $X$ is an arbitrary system of final state particles, can be described by,

$$
\sigma\left(P_{1}, P_{2}\right)=\sum_{i, j} \int d x_{1} d x_{2} f_{i}\left(x_{1}, \mu^{2}\right) \cdot f_{j}\left(x_{2}, \mu^{2}\right) \cdot \hat{\sigma}_{i j}\left(p_{1}, p_{2}, \alpha_{\mathrm{S}}\left(\mu^{2}\right), Q^{2} / \mu^{2}\right)
$$

where the indices $i, j$ run over the different parton species within the hadron [8]. This process is shown in diagrammatic form in Figure 3.3. The quantities $x_{1,2}$ denote the fractions of the initial hadron momenta carried by the partons participating in the hard interaction. Only the longitudinal component of the initial state hadron momentum is considered and the small transverse component is neglected. The momentum of an interacting parton $p$ is then given by $p=x P$. The functions $f_{i}\left(x, \mu^{2}\right)$ are known as parton distribution functions (PDF) and represent the probability 
density of finding a parton of species $i$ with momentum fraction $x$ within the parent hadron at a given factorisation scale $\mu$. The partonic cross section $\hat{\sigma}_{i j}$ is the cross section for the scattering of the two partons $i$ and $j$ to produce the system $X$. The quantity $Q$ is the characteristic scale of the hard scattering, for example the mass of the heavy quark in quarkonium production. The factorisation scale $\mu$ is the typical scale below which long distance (low energy) effects dominate and above which short distance (high energy) effects dominate. The factorisation scale is often chosen to be the same as the hard scattering energy scale, $\mu=Q$ [8]. This definition of $\mu$ allows the calculation of the total cross section to be factorised into a convolution between PDFs and a partonic cross section $\hat{\sigma}$. Parton behaviour at energy scales below $\mu$, such as the emission of soft gluons, is absorbed into the description of the PDF, negating the need for it to be considered in $\hat{\sigma}$. Through this approach, $\hat{\sigma}$ needs only to describe hard scattering processes for a reliable calculation of inelastic cross sections to be made. The partonic cross section describing the hard parton scattering can be reliably calculated perturbatively, since the strong coupling at the hard scattering energy scale $Q$ is weak due to asymptotic freedom. It should be noted that the majority of the total inelastic cross section for hadron-hadron interactions represents only soft parton interactions leading to low energy final states. These events must be described in an alternative manner. However, the production of a heavy quark pair (leading to quarkonium production) is a process that is typically well described by the factorisation model.

Examples of proton PDFs for the various parton species are shown in Figure 3.4, calculated at two different scales. As expected from the quark model of the proton, much of the momentum of the proton is carried by the $u$ and $d$ valence quarks, but this does not account for all of the proton's momentum. Fluctuations in the QCD vacuum can lead to the production of $q \bar{q}$ pairs of any flavour within the proton at sufficiently high $Q^{2}$. These sea quarks do not carry much of the proton momentum but populate a significant fraction of the low $x$ phase space as $Q^{2}$ increases. The 


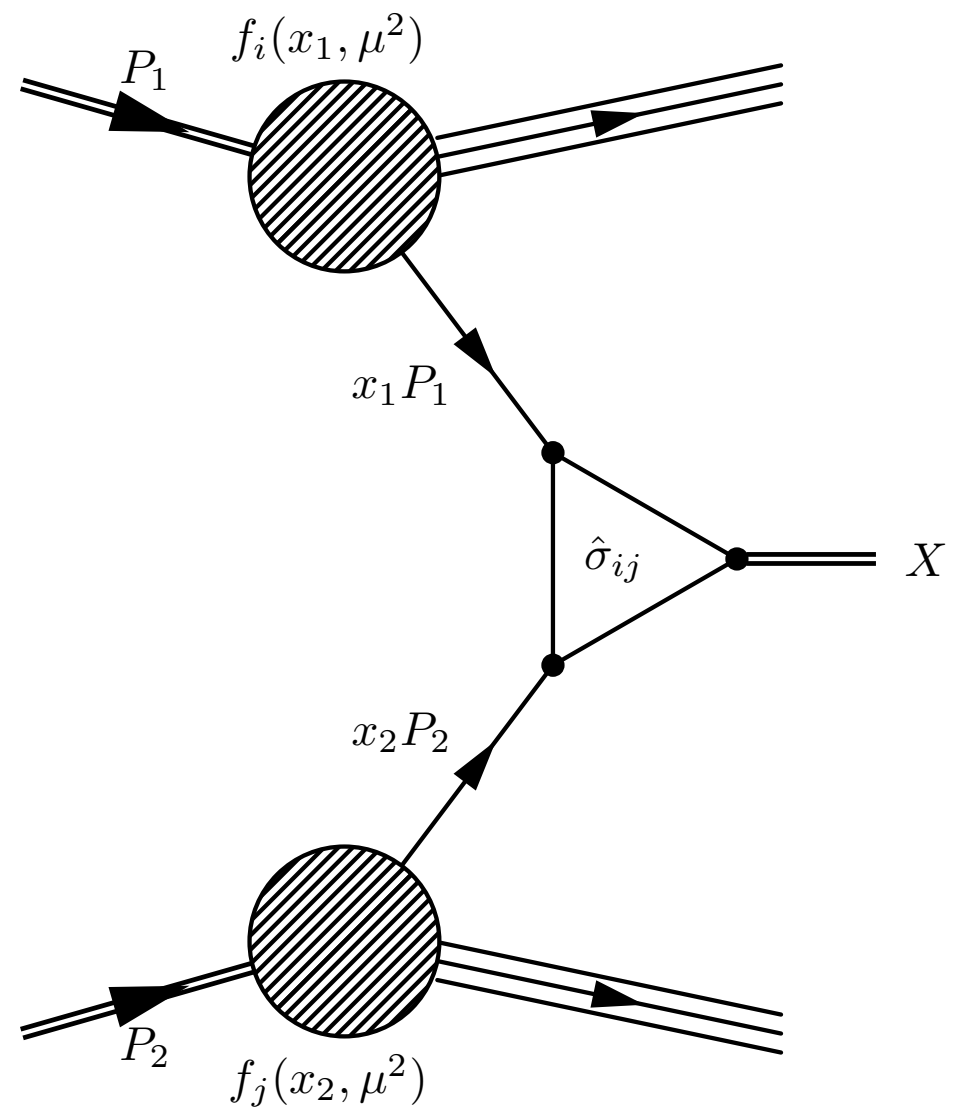

Figure 3.3: A diagram of hard parton-parton scattering within a hadron-hadron collision.

remaining significant fraction of the proton's momentum is carried by low $x$ gluons. In fact, gluons constitute the dominant contribution in the lowest $x$ region. This is an important feature of the PDF for quarkonium production at a hadron collider. Quarkonium production at central rapidity is typically a low $x$ and low $Q^{2}$ process. The momentum transfers $x_{1,2}$ required for a system of mass $M$ at a given centreof-mass (CM) energy $\sqrt{s}$ are given by $M=\sqrt{x_{1} x_{2} s}$. For example, at the LHC at $\sqrt{s}=7 \mathrm{TeV}$, the production of a $J / \psi$ with a mass around $3.1 \mathrm{GeV}$ imposes the constraint $x_{1} x_{2} \approx 2 \times 10^{-7}$. As a consequence of this, gluon initiated quarkonium production forms the dominant contribution to the total production cross section at high energy hadron colliders.

PDFs are fitted using experimental data. Data collected in a particular process (e.g. jet production at the HERA ep collider) will probe only a particular region of 
the $Q^{2}$ vs. $x$ phase space. PDFs can only be useful tools to calculate cross sections if they are known over a wide range of the $Q^{2}$ vs. $x$ phase space, not necessarily the regions where they are measured experimentally. However, the evolution of the PDFs in $Q^{2}$ is known from the Dokshitzer-Gribov-Lipatov-Altarelli-Parisi (DGLAP) equations [28-30]. Thus, a PDF measured as a function of $x$ at a particular value of $Q^{2}$ can be evolved to an arbitrary $Q^{2}$ (within the perturbative regime and at the expense of some theoretical uncertainty) with the DGLAP equations, allowing cross section predictions to be made in regions of phase space that have not yet been studied experimentally.
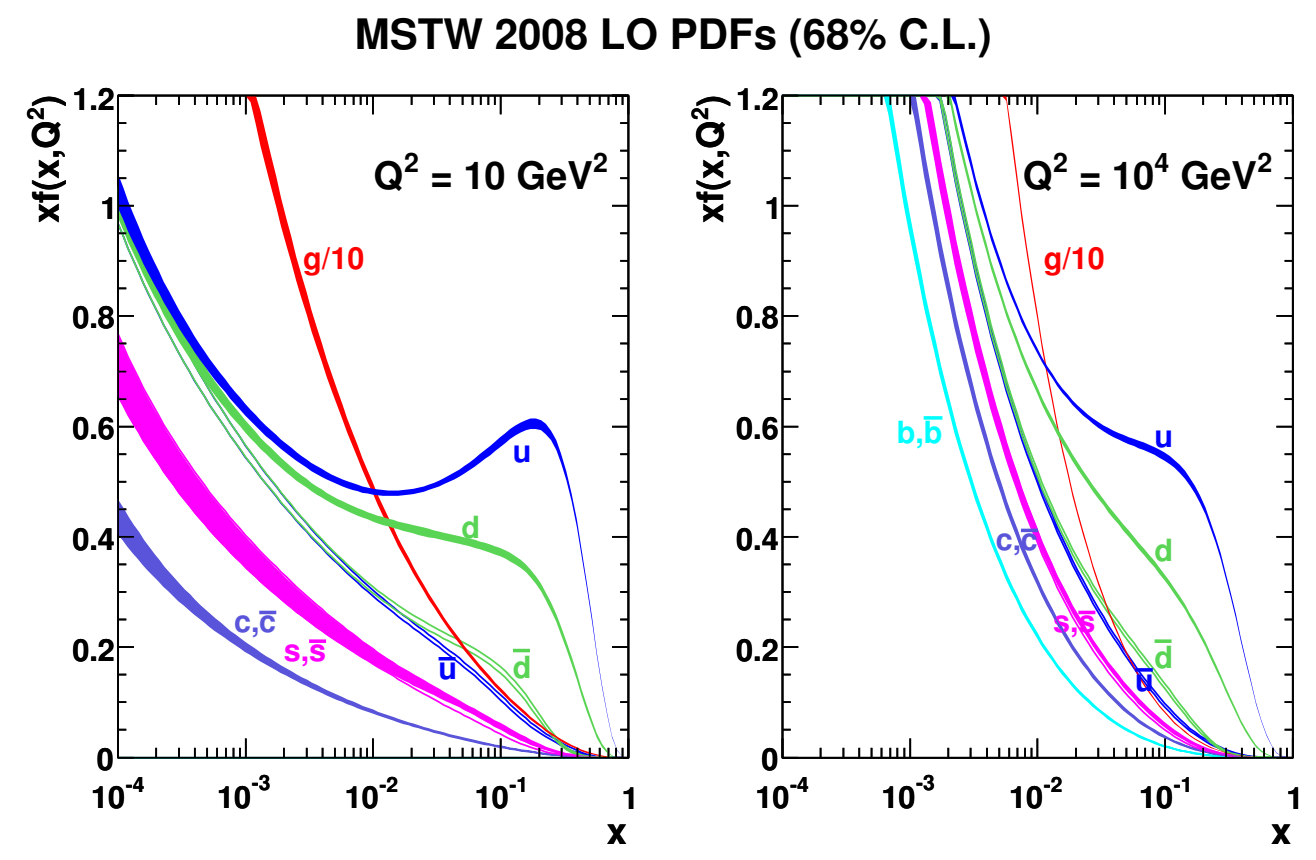

Figure 3.4: Proton parton distribution functions (PDF), calculated at leading order (LO) in perturbative QCD, for the various parton species calculated by the MSTW group [31]. The PDFs are evaluated at the scales of $Q^{2}=10 \mathrm{GeV}^{2}$ and $Q^{2}=$ $10^{4} \mathrm{GeV}^{2}$. The gluon PDF has been scaled down by a factor of 10 . Figure from [32].

Several different approaches have been developed to calculate the partonic cross section, $\hat{\sigma}(i j \rightarrow \mathcal{Q}+X)$, which describes the formation of a quarkonium state $\mathcal{Q}$ through the interaction of the partons $i$ and $j$. The most phenomenologically successful models will be described in Sections 3.2.2 to 3.2.5. 


\subsubsection{Colour Evaporation Model}

The Colour Evaporation Model (CEM) is an early model of quarkonium production (proposed only a few years after the discovery of the $J / \psi$ ) but has proved to be rather successful in describing the main features of quarkonium production in hadronic collisions [33-35]. The main ansatz of the CEM is that any $Q \bar{Q}$ pair produced in an hadronic collision will evolve into a quarkonium state if the $Q \bar{Q}$ invariant mass is below the relevant open-flavour threshold $(D \bar{D}$ for charmonium and $B \bar{B}$ for bottomonium). Further to this, it is assumed that the $Q \bar{Q}$ will evolve into a quarkonium state regardless of its spin and colour configuration. Soft gluon interactions (inconsequential to the bulk kinematics of the $Q \bar{Q}$ pair) are assumed to provide the mechanism by which a $Q \bar{Q}$ pair in an arbitrary spin and colour configuration can become arranged into a state with the same quantum numbers as a quarkonium state. For $Q \bar{Q}$ pairs with an invariant mass below the open-flavour threshold, the subsequent probability of the pair to evolve into a particular quarkonium state $\mathcal{Q}$ is given by the fraction $F_{\mathcal{Q}}$ [17], which is a phenomenologically determined constant, with no dependence on kinematic variables, the quantum numbers of the $Q \bar{Q}$ pair or indeed the $Q \bar{Q}$ production mechanism. While the fractions $F_{\mathcal{Q}}$ must be extracted from experimental data, they are assumed to be universal (i.e. process independent). Table 3.3 shows the experimentally determined $F_{\mathcal{Q}}$ parameters for various charmonium and bottomonium states. The resulting model contains no free parameters and is thus very predictive. The leading order cross section for the inclusive production of a quarkonium state $\mathcal{Q}$ in a $p p$ collision is given by,

$\sigma_{\mathrm{CEM}}(p p \rightarrow \mathcal{Q}+X)=F_{\mathcal{Q}} \sum_{i, j} \int_{4 m_{Q}^{2}}^{4 M_{Q}^{2}} d \hat{s} \int d x_{1} d x_{2} f_{i}\left(x_{1}, \mu\right) f_{j}\left(x_{2}, \mu\right) \hat{\sigma}_{i j}^{Q \bar{Q}}(\hat{s}) \delta\left(\hat{s}-x_{1} x_{2} s\right)$

where $m_{Q}$ is the mass of the heavy quark $Q, M_{Q}$ is the mass of the lightest meson containing the heavy quark $Q$ [17], $\hat{\sigma}_{i j}^{Q \bar{Q}}$ is the partonic cross section for $i j \rightarrow Q \bar{Q}$ 
production (where the indices $i, j$ run over the parton species) and $\sqrt{\hat{s}}$ is the partonic centre-of-mass energy. The partonic cross section for $i j \rightarrow Q \bar{Q}$ production is typically calculated in perturbative QCD.

\begin{tabular}{|c|c||c|c|}
\hline Charmonium State $\mathcal{Q}$ & $F_{\mathcal{Q}}^{\text {dir. }} / F_{J / \psi}^{\text {inc. }}$ & Bottomonium State $\mathcal{Q}$ & $F_{\mathcal{Q}}^{\text {dir. }} / F_{\Upsilon(1 S)}^{\text {inc. }}$ \\
\hline$J / \psi$ & 0.62 & $\Upsilon(1 S)$ & 0.52 \\
$\psi(2 S)$ & 0.14 & $\Upsilon(2 S)$ & 0.33 \\
$\chi_{c 1}$ & 0.60 & $\Upsilon(3 S)$ & 0.20 \\
$\chi_{c 2}$ & 0.99 & $\chi_{b}(1 P)$ & 1.08 \\
- & - & $\chi_{b}(2 P)$ & 0.84 \\
\hline
\end{tabular}

Table 3.3: The CEM parameters $F_{\mathcal{Q}}^{\text {dir. }}$ for the direct production of several charmonium and bottomonium states relative to the inclusive parameters $F_{J / \psi}^{\text {inc. }}$ and $F_{\Upsilon(i S)}^{\text {inc. }}$ respectively. The inclusive parameters take values of $F_{J / \psi}^{\text {inc. }}=0.0144-0.0248$ and $F_{\Upsilon(1 S)}^{\text {inc. }}=0.0201-0.0508$ where uncertainties in quark masses and PDFs are responsible for the ranges [17]. Parameter values are taken from Refs. [17,36].

More recent implementations of the CEM have been successful in describing the general features of charmonium production at the Tevatron [37]. However, in other respects the CEM stands in stark contrast with experimental observations. For example, the cross section for $\chi_{c}$ production relative to $J / \psi$ production is considered to be a universal constant, irrespective of the $Q \bar{Q}$ production mechanism. Contrary to this expectation, the available hadroproduction and photoproduction data on this ratio are not in good agreement [17]. The soft interactions that modify the quantum numbers of the initial $Q \bar{Q}$ system are assumed to lead to a uniform distribution of quarkonium spin states, with no particular configuration being preferred. This in turn predicts that the relative direct production rates for quarkonium states with the same orbital angular momentum quantum number, $L$, are determined by a simple counting of the allowed spin states [17]. For example, this suggests that the direct production rates of the $\chi_{c J}$ states should satisfy the ratios $1: 3: 5$ for the $J=0,1,2$ states, respectively. This feature is not observed experimentally; in fact more $\chi_{c 1}$ is observed to be produced relative to $\chi_{c 2}$ in hadronic collisions, 
even after feed-down from $\psi(2 S)$ decays is considered [38-40]. Overall, while the CEM successfully reproduces the general characteristics of quarkonium production in hadronic collisions, it consistently fails to describe the majority of the experimental data adequately on a quantitative level [41].

\subsubsection{Colour Singlet Model}

The colour-singlet model (CSM) is one of the earliest models of quarkonium production and centres around the idea that a $Q \bar{Q}$ pair is produced with the same quantum numbers as the quarkonium state into which it subsequently evolves [42-45]. Any physical hadronic state is required to be a colour singlet, and thus in the CSM, the $Q \bar{Q}$ pair must be directly produced in a colour singlet state and possessing the spin and angular momentum quantum numbers of the quarkonium state that it will eventually form. The probability for a $Q \bar{Q}$ pair to evolve into a quarkonium state is determined from the values of the colour singlet $Q \bar{Q}$ wavefunction (and its spatial derivatives) evaluated at the origin. These quantities are determined from potential models of the $Q \bar{Q}$ system and are constrained with experimental data on quarkonium decay widths (also related to the wavefunction at the origin). Other than the input on the $Q \bar{Q}$ wavefunction, the CSM contains no free parameters and is thus very predictive [17]. The partonic cross section for the production of a quarkonium state $\mathcal{Q}$ with quantum numbers ${ }^{2 S+1} L_{J}$ is given in the CSM by

$$
\hat{\sigma}_{\mathrm{CSM}}\left(i j \rightarrow \mathcal{Q}\left[{ }^{2 S+1} L_{J}\right]+X\right)=\left|\frac{d^{L} \Psi_{n l}(0)}{d r^{L}}\right|^{2} \tilde{\sigma}_{i j}\left(i j \rightarrow Q \bar{Q}\left[{ }^{2 S+1} L_{J}\right]\right),
$$

where $\Psi_{n l}$ is the $Q \bar{Q}$ wavefunction and $\tilde{\sigma}_{i j}$ is the partonic cross section for the interaction of the partons $i$ and $j$ to produce a colour singlet $Q \bar{Q}$ pair with the quantum numbers ${ }^{2 S+1} L_{J}[46]$.

The CSM enjoyed some success in predicting quarkonium production until ex- 
perimental data from the Tevatron suggested that the CSM significantly underestimated the prompt charmonium cross section in $p \bar{p}$ collisions at $\sqrt{s}=1.8$ [47] (see Figure 3.6). The CSM encounters further difficulties in predicting the production and decay of quarkonium states with non-zero orbital angular momentum, such as the $L=1 \chi$ states. Such calculations lead to infrared divergences that can only be cancelled through the inclusion of colour-octet contributions [17]. However, several modern proponents of the model exist, with recent calculations, including nextto-leading (NLO) and next-to-next-to-leading (NNLO) corrections in $\alpha_{\mathrm{S}}$, enjoying improved agreement with experimental data [48]. However, the sizes of these higher order corrections are found to be large, leading to concerns that the perturbative series may not be convergent [25]. As a result of these inconsistencies and its lack of experimental support, many consider the CSM no longer to be a theoretically robust model of quarkonium production.

\subsection{4 $k_{\mathrm{T}}$ Factorisation}

The $k_{\mathrm{T}}$ factorisation method is based upon an alternative procedure used to calculate the inclusive hadron-hadron scattering cross sections, distinct from that discussed in Section 3.2.1. The usual approach considers only the longitudinal momentum of the initial state partons and assumes that they possess zero initial transverse momentum; this is known as the collinear factorisation approach. The $k_{\mathrm{T}}$ factorisation approach uses alternative PDFs that include an explicit transverse momentum dependence, known as unintegrated parton distribution functions ( $\mathrm{UPDF}$ ). These $k_{\mathrm{T}}$ dependent uPDFs are often coupled with a partonic cross section calculated with the CSM. The proponents of this method argue that some of the shortcomings of the CSM are related to the approximations of collinear factorisation and that the use of $k_{\mathrm{T}}$ dependent uPDFs can remedy this [49]. Several predictions exist that significantly improve the agreement of the leading order (LO) CSM with experimental data [50, 51]. However, $k_{\mathrm{T}}$ dependent uPDFs (particularly the gluon distributions) suffer from 
much larger uncertainties than conventional PDFs and are not well constrained by experimental data.

\subsubsection{NRQCD Factorisation}

NRQCD factorisation represents the most successful approach to predicting quarkonium production, both in terms of its ability to describe many key experimental results and its theoretical completeness in comparison to earlier approaches [52].

One of the theoretical challenges associated with describing quarkonium production within QCD is the presence of multiple important energy-momentum scales. The heavy quark mass and parton hard-scattering momentum scales are generally significantly larger than $\Lambda_{\mathrm{QCD}}$. At these scales $\alpha_{\mathrm{S}}$ is generally small enough that perturbative methods can be used. However, other important effects involve inherently low energy processes (such as the evolution of a $Q \bar{Q}$ pair into a physical quarkonium state), which cannot be calculated perturbatively.

The energy-momentum scales relevant to quarkonium production include: the mass of the heavy quark, $m_{Q}$; the typical momentum of the heavy quark in the CM frame of a $Q \bar{Q}$ bound state, $m_{Q} v$ (where $v$ is the velocity of a heavy quark in the CM frame); and the typical binding energy of the $Q \bar{Q}$ pair, approximately $m_{Q} v^{2}$ [25]. For charmonium and bottomonium, the heavy quark CM velocity is sufficiently low $\left(v^{2} \approx 0.3\right.$ and $v^{2} \approx 0.1$, respectively) that non-relativistic approximations are valid. The final relevant momentum scale is the hard scattering scale, $Q^{2}$, which is typically given by the transverse momentum of the produced quarkonium state in hadronic collisions. The momentum scales $m_{Q}$ and $Q^{2}$ are related to short distance effects such as the formation of a $Q \bar{Q}$ pair while the scales $m_{Q} v$ and $m_{Q} v^{2}$ are associated with long distance effects such as hadron formation.

In order to make use of well-founded perturbative calculational techniques, the high momentum (short distance) effects that can be calculated within perturbation theory must be separated from the low momentum effects (long distance) which 
cannot. This is known as the "factorisation" of effects based on their momentum scale [17]. This factorisation can be achieved through the use of the effective field theory of non-relativistic QCD (NRQCD) [53]. An effective field theory can be considered as an approximate theory which contains only the degrees of freedom necessary to describe phenomena up to a particular scale. The effective theory of NRQCD can be shown to reproduce the results of QCD at momentum scales of $m_{Q} v$ and below [17].

The inclusive cross section for the direct production of a quarkonium state $\mathcal{Q}$ in proton proton collisions takes the form of a sum over products of short distance coefficients $\tilde{\sigma}_{n}\left[p p \rightarrow Q \bar{Q}^{[n]}\right]$ and long distance matrix elements (LDMEs) $\left\langle Q\left|\mathcal{O}_{n}\right| Q\right\rangle=\left\langle\mathcal{O}_{n}^{\mathcal{Q}}(\Lambda)\right\rangle$,

$$
\sigma\left(p p \rightarrow \mathcal{Q}+X^{\prime}\right)=\sum_{n} \tilde{\sigma}_{n}\left[p p \rightarrow Q \bar{Q}^{[n]}+X\right](\Lambda) \cdot\left\langle\mathcal{O}_{n}^{\mathcal{Q}}(\Lambda)\right\rangle
$$

where the parameter $\Lambda$ represents the high energy cut-off scale of the effective theory. The $\tilde{\sigma}_{n}\left[p p \rightarrow Q \bar{Q}^{[n]}\right]$ represent the short distance production cross sections for a $Q \bar{Q}$ pair with colour, spin and angular momentum quantum numbers, $n$, and can generally be calculated with perturbative QCD. One important feature of this approach is that the $Q \bar{Q}$ pair need not be produced with the same quantum numbers (colour, spin and angular momentum) as the quarkonium state into which it will evolve. Crucially, this allows the $Q \bar{Q}$ pair to be produced in either a colour singlet or a colour octet configuration.

The LDMEs are vacuum expectation values of the four-fermion NRQCD operators, $\mathcal{O}_{n}$, which represent the probabilities for a $Q \bar{Q}$ pair with a given set of colour, spin and angular momentum quantum numbers, $n$, to evolve into the quarkonium state $\mathcal{Q}$ plus anything (the quantum numbers of $\mathcal{Q}$ need not be identical to that of the $Q \bar{Q}$ pair). These matrix elements encode all of the non-perturbative physics associated with the evolution of a $Q \bar{Q}$ pair into a quarkonium state [25]. One useful property of the long-distance matrix elements is that they are process 
independent [17]. While this property has not been proven unambiguously, it is phenomenologically very useful and adds to the predictive power of the NRQCD factorisation approach. However, it is not yet known whether the LDMEs needed for the calculation of quarkonium production can be directly calculated from theory (i.e. with lattice QCD simulations). All present calculations in NRQCD use LDMEs extracted phenomenologically, by fitting experimental data.

The sum in the factorisation formula shown in Equation 3.9 can be parametrised as an expansion in powers of $\alpha_{\mathrm{S}}$ and $v$. Calculations in the NRQCD factorisation approach are performed by truncating this expansion at a fixed order in $v$. After truncation, only a finite number (of the infinite number in Equation 3.9) of unknown LDMEs contribute, making phenomenological predictions possible [25]. The CSM discussed in Section 3.2.3 can be derived from NRQCD factorisation by considering only the colour singlet term from Equation 3.9, at leading order in $v$, in which the quantum numbers of the $Q \bar{Q}$ pair are the same as the quarkonium state $\mathcal{Q}[25]$.

The predictive power of the NRQCD factorisation approach relies upon the availability of a set of LDMEs that are complete at a given power in $v$ which also provide useful predictions (i.e. beyond trivial case of the CSM). The symmetries of NRQCD predict several approximate relationships between different LDMEs, which reduces the total number of independent free parameters needed at a given order in $v[17,52]$.

In the case of operators that have the same $Q \bar{Q}$ angular momentum and colour quantum numbers as the dominant Fock state (eigenstates of the particle number operator) of the corresponding quarkonium state, an approximate relationship exists (up to corrections in $v$ ) between some LDMEs and the $Q \bar{Q}$ wavefunctions (and their derivatives) evaluated at the origin (as used in the CSM and in potential models of the quarkonium spectrum) [17]. Two examples of this relationship are shown in Equations 3.10 and 3.11, which relate the colour singlet matrix elements for the dominant Fock state for the $J / \psi$ and $\chi_{c J}$ to their wavefunctions $\Psi_{\mathcal{Q}}$ (and derivatives) and a colour factor. These relationships allow the direct extraction of the CSM, 
formulated in terms of the $Q \bar{Q}$ wavefunctions, from NRQCD.

$$
\begin{gathered}
\left\langle\mathcal{O}_{1}^{J / \psi}\left({ }^{3} S_{1}\right)\right\rangle=\frac{3 N_{c}}{2 \pi}\left|\Psi_{J / \psi}(0)\right|^{2}\left(1+\mathcal{O}\left(v^{4}\right)\right) \\
\left\langle\mathcal{O}_{1}^{\chi} \chi_{c J}\left({ }^{3} P_{J}\right)\right\rangle=(2 J+1) \frac{3 N_{c}}{2 \pi}\left|\Psi_{\chi_{c J}}^{\prime}(0)\right|^{2}\left(1+\mathcal{O}\left(v^{2}\right)\right)
\end{gathered}
$$

The simplest yet non-trivial truncation in $v$ yields four independent matrix elements for an $S$-wave multiplet $\left(J / \psi\right.$ and $\left.\eta_{c}\right)$ and two independent matrix elements for a $P$-wave multiplet $\left(\chi_{c J}\right.$ and $\left.h_{c}\right)$ [17]. The typical choice of $S$-wave matrix elements is $\left\langle\mathcal{O}_{1}^{J / \psi}\left({ }^{3} S_{1}\right)\right\rangle,\left\langle\mathcal{O}_{8}^{J / \psi}\left({ }^{1} S_{0}\right)\right\rangle,\left\langle\mathcal{O}_{8}^{J / \psi}\left({ }^{3} S_{1}\right)\right\rangle$ and $\left\langle\mathcal{O}_{8}^{J / \psi}\left({ }^{3} P_{0}\right)\right\rangle$, which enter the expansion in $v$ at orders $1, v^{3}, v^{4}$ and $v^{4}$ respectively [17]. The $P$-wave matrix elements are typically chosen to be $\left\langle\mathcal{O}_{1}^{\chi} \chi_{c 0}\left({ }^{3} P_{0}\right)\right\rangle$ and $\left\langle\mathcal{O}_{8}^{\chi}{ }_{c 0}\left({ }^{3} S_{1}\right)\right\rangle$, which both contribute at order $v^{2}$ [17]. Together, these matrix elements can be used to calculate the production cross sections for all of the spin states in the $S$-wave and $P$-wave multiplets and can thus provide specific predictions for the polarisation of the quarkonium states. It should be noted that separate matrix elements exist for each set of radial excitations, $n$. For example, the matrix elements for $\psi(2 S)$ production differ from the equivalent matrix elements for $J / \psi$ production and must be obtained separately. Many physical observables that can be extracted from experimental data are only sensitive to a linear combination of colour octet matrix elements. The linear combination,

$$
M_{k}^{\mathcal{Q}}=\left\langle\mathcal{O}_{8}^{\mathcal{Q}}\left({ }^{1} S_{0}\right)\right\rangle+\frac{k}{m_{Q}}\left\langle\mathcal{O}_{8}^{\mathcal{Q}}\left({ }^{3} P_{0}\right)\right\rangle
$$

is often defined to facilitate the extraction of these matrix elements from data (an appropriate value of $k$ is chosen depending on the experimental observable). The matrix elements for charmonium are reasonably well known. Table 3.4 shows a set of NRQCD matrix elements extracted from charmonium production cross sections (differential in transverse momentum) measured by CDF [47,54], taken from Ref. [55]. The situation for bottomonium production is less clear due to the compar- 
ative lack of data and the large and complex feed-down contributions to the $\Upsilon(n S)$ cross sections.

\begin{tabular}{|c|c|c|c|}
\hline \hline \multirow{2}{*}{$J / \psi$} & $\left\langle\mathcal{O}_{1}^{J / \psi}\left({ }^{3} S_{1}\right)\right\rangle$ & $\left\langle\mathcal{O}_{8}^{J / \psi}\left({ }^{3} S_{1}\right)\right\rangle$ & $M_{3.5}^{J / \psi}$ \\
\cline { 2 - 4 } & $1.16 \mathrm{GeV}^{3}$ & $(1.19 \pm 0.14) \times 10^{-2} \mathrm{GeV}^{3}$ & $(4.54 \pm 1.11) \times 10^{-2} \mathrm{GeV}^{3}$ \\
\hline \hline \multirow{3}{*}{$\psi(2 S)$} & $\left\langle\mathcal{O}_{1}^{\psi(2 S)}\left({ }^{3} S_{1}\right)\right\rangle$ & $\left\langle\mathcal{O}_{8}^{\psi(2 S)}\left({ }^{3} S_{1}\right)\right\rangle$ & $M_{3.5}^{\psi(2 S)}$ \\
\cline { 2 - 4 } & $0.76 \mathrm{GeV}^{3}$ & $(0.50 \pm 0.06) \times 10^{-2} \mathrm{GeV}^{3}$ & $(1.89 \pm 0.46) \times 10^{-2} \mathrm{GeV}^{3}$ \\
\hline \hline \multirow{2}{*}{$\chi_{c 0}$} & $\left\langle\mathcal{O}_{1}^{\chi} \chi_{c 0}\left({ }^{3} P_{0}\right)\right\rangle$ & $\left\langle\mathcal{O}_{8}^{\chi}{ }^{20}\left({ }^{3} S_{1}\right)\right\rangle$ & - \\
\cline { 2 - 4 } & $0.11 \mathrm{GeV}^{3}$ & $(0.31 \pm 0.04) \times 10^{-2} \mathrm{GeV}^{3}$ & - \\
\hline \hline
\end{tabular}

Table 3.4: NRQCD matrix elements for charmonium production extracted from CDF data $[47,54]$, taken from Ref. [55]

\subsubsection{Charmonium production in b-hadron decay}

The decays of hadrons containing $b$ quarks represent a significant contribution to charmonium production in hadronic collisions. No analogous process contributes to bottomonium production due to the absence of hadrons containing top quarks. Bottom quarks, and thus b-hadrons, are copiously produced at high energy hadron colliders and the typical inclusive branching fractions for the decays of $b$-hadrons $\left(H_{b}\right)$ to final states including charmonium states $\mathcal{C}, \mathcal{B}\left(H_{b} \rightarrow \mathcal{C}+X\right)$ are of order $10^{-3}$. The fragmentation of $b$ quarks tends to produce a mixture of ground state and excited $b$-hadrons (such as the $B^{*(*)}$ mesons). The excited states quickly decay to the ground state mesons, $B^{ \pm}, B^{0}, B_{s}^{0}$, and the weakly decaying $b$-baryons (e.g. $\Lambda_{b}$ ), all of which can subsequently decay to final states involving a charmonium state. The fraction of $b$ quarks that fragment into $B_{c}$ mesons is expected to be very small $(\sim 0.2 \%$ from Tevatron measurements) [3]. 
Charmonium production from $b$-hadron decays produced in hadron collisions is typically described with a phenomenological model consisting of a $b$ quark production cross section calculated in perturbative QCD, coupled with a phenomenological or data-driven description of the $b$ quark fragmentation process and $b$-hadron decay [56]. Thus, the total cross section for the production of the charmonium state $\mathcal{C}$ from $b$-hadron decay in $p p$ collisions can be given by,

$$
\sum_{i}\left[\tilde{\sigma}\left(p p \rightarrow b+X^{\prime}\right) \otimes f\left(b \rightarrow H_{b}^{i}\right) \otimes D\left(H_{b}^{i} \rightarrow \mathcal{C}+X\right)\right] \cdot \mathcal{B}\left(H_{b}^{i} \rightarrow \mathcal{C}+X\right),
$$

where the index $i$ runs over the relevant weakly decaying $b$-hadrons and the $\otimes$ symbol represents a convolution in momentum [56]. The cross section $\tilde{\sigma}$ describes the inclusive production of $b$ quarks in $p p$ collisions and is typically calculated with perturbative QCD. The fragmentation functions $f\left(b \rightarrow H_{b}\right)$ give the probability for a $b$ quark to produce a $b$-hadron $H_{b}$ with a fraction $z$ of the initial $b$ quark momentum. These functions typically contain a single free parameter which is determined by fitting experimental data. Typical analytical forms include the Kartvelishvili function shown in Equation 3.14 and the Peterson function shown in Equation 3.15 [57,58].

$$
\begin{gathered}
f(z)=z^{\alpha}(1-z) \\
f(z)=\frac{1}{z}\left(1-\frac{1}{z}-\frac{\epsilon_{\mathrm{P}}}{1-z}\right)^{-2}
\end{gathered}
$$

The $b$ quark fragmentation functions have been precisely measured by the LEP experiments in $Z \rightarrow b \bar{b}$ decays. Typical fitted values for the Peterson and Kartvelishvili parameters are $\epsilon_{\mathrm{P}}=41.2 \times 10^{-4}$ and $\alpha=11.9$ [59]. The fragmentation functions are generally only measured for the inclusive mixture of $b$-hadrons produced in $Z \rightarrow b \bar{b}$ decays and not separately for each individual $b$-hadron species. The measured $b$ hadron mixtures produced in $Z \rightarrow b \bar{b}$ decays at LEP and in $p \bar{p} \rightarrow b \bar{b}+X$ production 
at the Tevatron are shown in Table 3.5. There is a difference between the LEP and Tevatron results, suggesting that the fractions may have some dependence on the average $b$-quark momentum (around $p \sim m_{Z} / 2$ at LEP, but typically lower at the Tevatron).

\begin{tabular}{|c|c|c||c|}
\hline \multirow{2}{*}{$b$-hadron } & \multicolumn{3}{|c|}{ Fraction $[\%]$} \\
\cline { 2 - 4 } & LEP $Z \rightarrow b \bar{b}$ & Tevatron $p \bar{p} \rightarrow b \bar{b}+X$ & Combination \\
\hline$B^{ \pm}, B^{0}$ & $40.3 \pm 0.9$ & $33.9 \pm 3.9$ & $40.1 \pm 0.8$ \\
$B_{s}^{0}$ & $10.3 \pm 0.9$ & $11.1 \pm 1.4$ & $10.5 \pm 0.6$ \\
$b$-baryons & $9.0 \pm 1.5$ & $21.2 \pm 6.9$ & $9.3 \pm 1.6$ \\
\hline
\end{tabular}

Table 3.5: The mixture of $b$-hadrons measured in $Z \rightarrow b \bar{b}$ decays at LEP and $p \bar{p} \rightarrow b \bar{b}+X$ production at the Tevatron [3]. The fractions for $B^{ \pm}$and $B^{0}$ are considered to be equal (i.e. a factor of 2 is understood in this case such that all of the fractions to sum to unity).

The functions $D\left(H_{b} \rightarrow \mathcal{C}+X\right)$ are analogous to the $b$ quark fragmentation functions and describe the fraction of the $b$-hadron momentum carried by the charmonium state $\mathcal{C}$ in the decay $H_{b} \rightarrow \mathcal{C}+X$ and are often parametrised in terms of the momentum of the charmonium state in the rest frame of the decaying $b$-hadron, $p^{*}$. These distributions have been measured in the decays of the $B^{ \pm} / B^{0}$ meson mixture produced in $\Upsilon(4 S)$ decays at the B factories [60], though no data exist for inclusive $B_{s}^{0}$ or $b$-baryon decays.

The branching fractions for the inclusive production of charmonium states in the decays of the $B^{ \pm} / B^{0}$ meson mixture produced in $\Upsilon(4 S)$ decays have been precisely measured by the CLEO, BaBar and Belle experiments [3]. At hadron collider experiments, where the dominant source of $b$-hadron production is via $b$ quark fragmentation, the $b$-hadron mixture, and the corresponding inclusive charmonium branching fractions, are less well known. Several measurements of the branching fractions for the inclusive decays $\mathcal{B}(b \rightarrow J / \psi+X)$ and $\mathcal{B}(b \rightarrow \psi(2 S)+X)$ have been made for the $b$-hadron mixtures produced at LEP, the Tevatron and LHC, though little data exist for inclusive decays to the $\chi_{c}$ states (none in fact for $\chi_{c 2}$ ). Table 3.6 
summarises the world average measurements on inclusive branching fractions for the decays of $b$-hadron mixtures to final states including charmonium. While the inclusive branching fractions for decay to $J / \psi$ and $\psi(2 S)$ from $B^{ \pm}, B^{0}$ and from $B^{ \pm}, B^{0}, B_{s}^{0}, b$-baryon mixtures are compatible, the measurements do not agree for $\chi_{c 1}$, where the only $B^{ \pm}, B^{0}, B_{s}^{0}, b$-baryon measurements are from LEP. This may suggest that the $\chi_{c 1}$ inclusive branching fractions are more sensitive to the $B_{s}^{0}$ or $b$-baryon content of the $b$-hadron mixtures than are the $J / \psi$ and $\psi(2 S)$ inclusive branching fractions.

\begin{tabular}{|c|c|c|}
\hline \multirow{2}{*}{ State } & \multicolumn{2}{|c|}{ Inclusive Branching Fraction } \\
\cline { 2 - 3 } & $B^{ \pm / 0}(\Upsilon(4 S)$ Decays $)$ & $B^{ \pm / 0}, B_{s}^{0}, b$-baryon (LEP, Tevatron and LHC) \\
\hline$J / \psi$ & $(10.94 \pm 0.32) \times 10^{-3}$ & $(11.6 \pm 1.0) \times 10^{-3}$ \\
$\psi(2 S)$ & $(3.07 \pm 0.21) \times 10^{-3}$ & $(2.83 \pm 0.29) \times 10^{-3}$ \\
$\chi_{c 1}$ & $(3.86 \pm 0.27) \times 10^{-3}$ & $(14 \pm 4) \times 10^{-3}$ \\
$\chi_{c 2}$ & $(1.3 \pm 0.4) \times 10^{-3}$ & - \\
\hline
\end{tabular}

Table 3.6: Branching fractions for the inclusive decays of $b$-hadron mixtures to final states including charmonium. Data taken from Ref. [3].

The limited knowledge of the production fractions, fragmentation functions and inclusive branching fractions for the relevant $b$-hadron species in hadron collider experiments constrains the accuracy with which charmonium production from $b$ hadron decays can be predicted. Furthermore, the universality of the $b$-hadron production fractions and inclusive branching fractions measured at LEP and the hadron colliders has not been proven. Several measurements, particularly the $b$-hadron production fractions shown in Table 3.5, point towards a potential systematic discrepancy and potentially significant source of uncertainty that is often not considered. Nonetheless, modern predictions for charmonium production from $b$-hadron decay calculated within this semi-phenomenological framework have experienced much success in describing the data from the Tevatron and LHC experiments [56,61]. 


\subsubsection{Hadron Collider Quarkonium Production Phenomenol- ogy}

The inclusive production of quarkonium states in hadronic collisions can be separated into two distinct processes: direct production and feed-down. Direct production denotes the production of a quarkonium state "directly" in a hard scattering process, as described in the preceding discussion in this Chapter. Feed-down denotes the production of quarkonium states in the decay of other quarkonium states (or $b$-hadrons in the case of charmonium). The inclusive production of a given quarkonium state in hadronic collisions is often a complicated mixture of direct production and feed-down, particularly for the ground state quarkonia. The total feed-down contribution often represents many individual contributions from various decay chains (e.g. $\psi(2 S) \rightarrow J / \psi \pi^{+} \pi^{-}$and $\chi_{c J} \rightarrow J / \psi \gamma$ ) and is typically experimentally indistinguishable from direct production unless the full feed-down decay chain is reconstructed. However, in the case of charmonium production, one major feed-down contribution can be reliably separated. The contribution from the decays of $b$-hadrons can often be separated experimentally from the inclusive production cross section through an exploitation of the long lifetime of the weakly decaying b-hadrons with an analysis of a suitable decay time variable. The experimentally separated contribution from the decays of $b$-hadrons is often called the non-prompt contribution, while the remainder is referred to as the prompt contribution. The distinction between prompt and non-prompt charmonium production is important as the two processes are described theoretically within distinct calculational frameworks. Meaningful comparisons between experimental data and theoretical models of charmonium production can only be made if the prompt / non-prompt separation has been performed. This complication is absent in bottomonium production (though feed-down between bottomonium states is present). At hadron collider experiments, generally only the spin triplet $S=1$ quarkonium states are studied as 
they can decay directly to a di-lepton final state or can readily decay to a vector quarkonium state (i.e. the $\chi$ states). This situation occurs because the dominantly hadronic final states of the spin singlet $S=0$ quarkonia cannot be triggered upon due to the very large combinatorial backgrounds, while the di-lepton signature is very clean experimentally.

The most common quarkonium production observable is the cross section, measured either as an absolute quantity or differentially in an appropriate kinematic variable (typically the transverse momentum and/or (pseudo-)rapidity of the quarkonium state). Absolute and differential cross sections are often very sensitive to various production processes and are usually the primary means through which the validity of theoretical models is tested. The other important observable for quarkonium states with non-zero total angular momentum $J$ (e.g. the $J=1 J / \psi$ and $\Upsilon$ states) is the polarisation of the quarkonium. The polarisation is related to the angular momentum eigenstate, $J_{z}$, composition of a quarkonium state with respect to an axis $z$. In general, a quarkonium state $\mathcal{Q}$ with total angular momentum $J$ can be produced in a linear superposition of the allowed angular momentum eigenstates of $J_{z}$ given by,

$$
|\mathcal{Q}\rangle=\sum_{m=-J}^{J} a_{m}|\mathcal{Q} ; J, m\rangle,
$$

such that $\hat{J}_{z}|\mathcal{Q} ; J, m\rangle=m|\mathcal{Q} ; J, m\rangle$ and the coefficients $a_{m}$ satisfy $\sum_{m}\left|a_{m}\right|^{2}=1$. Different production mechanisms can lead to a preference for quarkonia to be produced in particular angular momentum eigenstates (measured with respect to an appropriate axis) due to angular momentum, parity and helicity conservation (in strong and EM interactions). This sensitivity to the production mechanism makes the quarkonium polarisation a very important observable that often provides information complementary to that accessed through cross section measurements. One example of this sensitivity is the production of a vector $\left(J^{P C}=1^{--}\right)$quarkonium state $V$ through gluon fragmentation $(g \rightarrow Q \bar{Q} \rightarrow V+X)$. In the case of an on shell 
gluon (which must have helicity \pm 1 ), to conserve angular momentum, the quarkonium state $V$ must be produced in a state where $J_{z}= \pm 1$ when measured along the axis of gluon propagation (approximately the direction of quarkonium propagation, since $X$ is typically soft).

Quarkonium polarisation is measured experimentally through an analysis of the angular distributions of the quarkonium decay products. The most common choices of decay for the measurement of quarkonium polarisation are the di-lepton decays of the vector quarkonium states $V \rightarrow \ell^{+} \ell^{-}$. The polarisations of the vector states can also be used to probe the polarisation of the $P$-wave $\chi$ states in the radiative decays $\chi \rightarrow V \gamma \rightarrow \ell^{+} \ell^{-} \gamma[62]$. Vector quarkonia are said to have a transverse polarisation if they are in a $J_{z}= \pm 1$ eigenstate or a longitudinal polarisation if in a $J_{z}=0$ eigenstate. Contrary to the nomenclature (adopted in analogy with the photon, and in reference to the electromagnetic field), the spin vector is aligned along $z$ for a transverse polarisation and perpendicular to $z$ for a longitudinal polarisation [63].

Figure 3.5 shows the typical system of axes and angles adopted in measurements of quarkonium polarisation. The polar angle, $\theta$, between the positive lepton and the polarisation axis, and the azimuthal angle, $\phi$, between the positive lepton (in the $V$ rest frame) and the production plane (also measured in the $V$ rest frame) are the typical angular observables used in quarkonium polarisation measurements in $V \rightarrow \ell^{+} \ell^{-}$decays.

Typical choices of the polarisation axis include the helicity frame (HX), defined as the quarkonium line of flight in the lab frame, the Collins-Soper frame, defined as the bisector of the angle between the two hadron momenta in the quarkonium rest frame, and the Gottfried-Jackson frame, defined as the direction of one of the hadron momenta in the quarkonium rest frame [63]. In the case of the inclusive production of $V$ in hadron collisions, the two dimensional angular distribution $W(\theta, \phi)$ is given by 


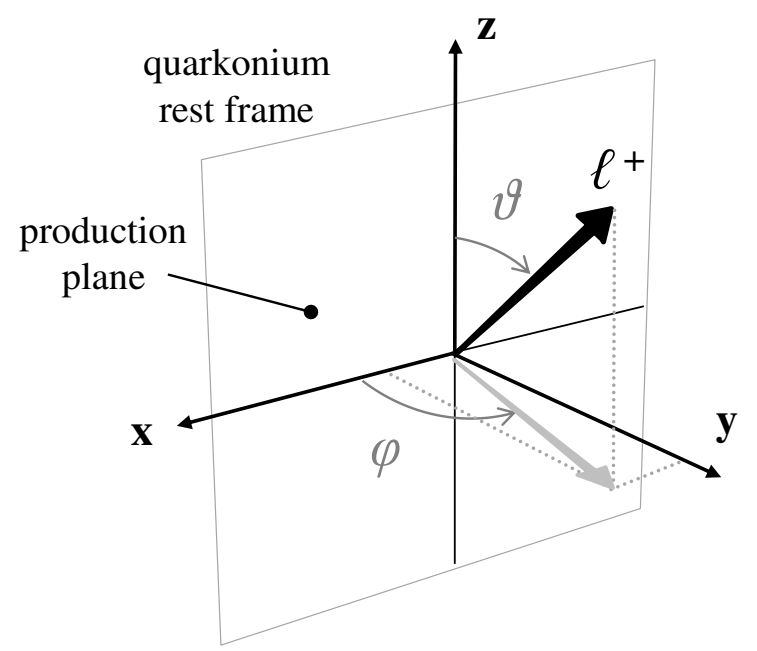

Figure 3.5: The angles relevant to the measurement of quarkonium polarisation in $V \rightarrow \ell^{+} \ell^{-}$decays. The angles $\theta$ and $\phi$ are defined in the quarkonium rest frame. The production plane is the plane which contains the momentum of the colliding hadrons. Various conventions determine the direction of the polarisation axis $z$. Figure from Ref. [63].

$$
W(\theta, \phi) \propto \frac{1}{\left(3+\lambda_{\theta}\right)}\left[1+\lambda_{\theta} \cos ^{2} \theta+\lambda_{\phi} \sin ^{2} \theta \cos 2 \phi+\lambda_{\theta \phi} \sin 2 \theta \cos \phi\right]
$$

where the $\lambda$ coefficients $(|\lambda| \leq 1)$ are related to the angular momentum eigenstate composition of the produced quarkonia. In the case of pure transverse polarisation $\left(J_{z}= \pm 1\right) \lambda_{\theta}=+1$ and $\lambda_{\phi}=\lambda_{\theta \phi}=0$, while for longitudinal polarisation $\lambda_{\theta}=-1$ and $\lambda_{\phi}=\lambda_{\theta \phi}=0$.

\section{Charmonium Production Phenomenology}

Charmonium production at hadron colliders is generally studied with the $J / \psi, \chi_{c J}$ and $\psi(2 S)$ states. The $J / \psi$ is the most studied state as it is the most accessible from an experimental perspective. The inclusive production of prompt $J / \psi$ (i.e. neglecting the experimentally separable contribution from $b$-hadron decays) is composed of three major contributions; direct production, feed-down from radiative $\chi_{c}$ decays and feed-down from the decays of the $\psi(2 S)$. The direct contribution 
is dominant and represents around $(64 \pm 6) \%$ of the cross section [54]. The radiative decays of $\chi_{c J} \rightarrow J / \psi \gamma$ contribute around $(25 \pm 5) \%$ while the hadronic decays $\psi(2 S) \rightarrow J / \psi+X$ (the inclusive branching fraction for such decays is $(60.3 \pm 0.7) \%[3])$ contribute around $(8.1 \pm 0.3) \%$ [64]. The $\chi_{c J}$ states are studied through their decays $\chi_{c J} \rightarrow J / \psi \gamma$ and the prompt cross section is predominately direct with a small feed-down contribution from $\psi(2 S) \rightarrow \chi_{c J} \gamma$ decays of around $5 \%$ of the total rate [38]. Prompt $\psi(2 S)$ production is almost entirely direct due to the absence of any higher mass states below the open-charm threshold.

\section{Bottomonium Production Phenomenology}

Bottomonium production at hadron colliders is studied with the $\Upsilon(n S)$ and $\chi_{b}(n P)$ states. The $\Upsilon(n S)$ states are the most studied (similar to the $J / \psi$ for charmonium). The feed-down contributions to the $\Upsilon(1 S)$ cross section are complicated and include contributions from the radiative decays of all the $\chi_{b}$ states and the hadronic decays of the $\Upsilon(2,3 S)$ states. The fraction of $\Upsilon(1 S)$ produced directly (in $p \bar{p}$ collisions at $\sqrt{s}=$ $1.8 \mathrm{TeV}$ ) has been measured by CDF to be only $51 \pm 16 \%$ [65]. The corresponding fraction for the prompt $\Upsilon(2 S)$ cross section has not been measured, but is likely to be similar given the large branching fractions for the decays $\chi_{b J}(2 P) \rightarrow \Upsilon(2 S) \gamma$ ) (for $J=1,2)$ and $\Upsilon(3 S) \rightarrow \Upsilon(2 S) X$, all of which are between 10-20\% [3]. The prompt $\Upsilon(3 S)$ cross section was once considered to be fully direct, in analogy with the $\psi(2 S)$, though the recent discovery of a candidate for the $\chi_{b}(3 P)$ states now suggests that a possibly significant feed-down contribution may exist $[66,67]$. The radiative decays $\Upsilon(n S) \rightarrow \chi_{b}((n-1) P) \gamma$ are the only dominant feed-down contributions to the

prompt $\chi_{b}(n P)$ cross sections. No data exist on the direct fractions of prompt $\chi_{b}$ production. Radiative $\Upsilon$ decays have branching fractions at the level of between $3-13 \%$ [3] (a rate similar to $\psi(2 S) \rightarrow \chi_{c J} \gamma$ ) and are only expected to contribute at a low level, similar to the situation for $\chi_{c}$ in the charmonium sector. 


\subsubsection{Quarkonium Production Measurements at the Teva- tron}

The cross sections for the production of prompt $J / \psi, \psi(2 S)$ and $\chi_{c}$ were measured by $\mathrm{CDF}$ in $p \bar{p}$ collisions at $\sqrt{s}=1.8 \mathrm{TeV}$ at the Tevatron in Run I (see Figure 3.6) $[47,54]$. All of the dominant contributions to prompt $J / \psi$ production were determined and an estimate of the direct $J / \psi$ production cross section was made. Production cross section measurements for the $J / \psi$ and $\psi(2 S)$ states were also performed with the much larger Tevatron Run II dataset collected at $\sqrt{s}=1.96 \mathrm{TeV}[68,69]$. The D0 experiment also performed similar measurements with the Tevatron Run I dataset [70]. The measured prompt $J / \psi$ cross sections were found to be over an order of magnitude greater than the expectations of the CSM, prompting a renaissance in theoretical models. NRQCD based predictions were subsequently found to be in much better agreement with the measurements. No measurements of the absolute $\chi_{c}$ production cross sections were performed with the Tevatron Run II dataset $(\sqrt{s}=1.96 \mathrm{TeV})$ though a measurement of the relative prompt production cross section $\sigma\left(\chi_{c 2}\right) / \sigma\left(\chi_{c 1}\right)$ was made [38]. This measurement was the first to contradict strongly the expectations of the CSM and CEM, measuring a $\chi_{c 1}$ cross section in excess of that of $\chi_{c 2}$. The prompt and non-prompt $J / \psi$ and $\psi(2 S)$ polarisations were measured with the Tevatron Run II dataset by CDF [71]. CDF measured a small longitudial polarisation for prompt $J / \psi$ production, in disagreement with the NRQCD prediction of a strong transverse polarisation which increases with transverse momentum.

The production cross sections for the $\Upsilon(n S)$ states in $\sqrt{s}=1.8 \mathrm{TeV} p \bar{p}$ collisions were measured by both D0 [72] and CDF [73] using the Tevatron Run I dataset. Both measurements are well described by NRQCD based predictions in the high transverse momentum region [55]. The polarisations of the $\Upsilon(n S)$ states were also measured by D0 [74] and CDF [75] with the Tevatron Run II dataset $(\sqrt{s}=1.96 \mathrm{TeV})$. 
Both experiments observed a longitudinal polarisation. CDF measured only a slight longitudinal polarisation for $\Upsilon(1 S)$ production $\left(-0.23<\lambda_{\theta}<0.01\right)$, though D0 measured a much stronger longitudinal polarisation with a contradictory transverse momentum dependence. The measurement of only the polar angle $\theta$ in $\Upsilon \rightarrow \mu^{+} \mu^{-}$ decays (integrating over the azimuthal angle $\phi$ ) and the use of frame dependent quantities has been suggested as the source of this discrepancy [63]. Neither of the measurements supports the NRQCD prediction of a transverse polarisation.

\subsubsection{Quarkonium Production Measurements at the LHC}

All of the LHC experiments have now contributed measurements of quarkonium production at a variety of CM energies. The production cross sections for both prompt and non-prompt $J / \psi$ have been measured by all of the LHC experiments in $p p$ collisions at $\sqrt{s}=7 \mathrm{TeV}[76-79]$ and at $\sqrt{s}=8 \mathrm{TeV}$ [80]. The CMS and LHCb experiments have also measured the prompt and non-prompt $\psi(2 S)$ production cross sections at $\sqrt{s}=7 \mathrm{TeV}[78,81]$. The ATLAS, CMS and LHCb $J / \psi$ and $\psi(2 S)$ differential cross section measurements are shown in Figures 3.7, 3.8 and 3.9 respectively. The fraction of prompt $J / \psi$ produced in the radiative decays $\chi_{c J} \rightarrow J / \psi \gamma$ was measured at $\sqrt{s}=7 \mathrm{TeV}$ to be between $14.0 \%$ at low $J / \psi$ transverse momentum $(3 \mathrm{GeV})$ rising to $26.8 \%$ at higher transverse momentum $(14 \mathrm{GeV})$ [82]. The production cross section of $\chi_{c 2}$ relative to $\chi_{c 1}$ has also been measured by CMS [40] and LHCb [39], confirming the CDF observation of $\sigma\left(\chi_{c 1}\right)>\sigma\left(\chi_{c 2}\right)$. In general, all of the prompt charmonium production cross sections measured at the LHC are well described by NRQCD predictions (with LDMEs extracted from Tevatron data). The ALICE, CMS and LHCb experiments have all measured the polarisation of promptly produced $J / \psi$ [83-85]. All of the experiments measure only a weak polarisation, consistent with zero in some regions of phase space. The CMS experiment has also measured the polarisation of promptly produced $\psi(2 S)$ to be similarly weak [83]. These observations further increase the disagreement with the NRQCD predictions 
of a strong transverse polarisation.

In addition to measurements of inclusive charmonium production, the $\mathrm{LHCb}$ experiment has measured the cross sections for the exclusive production of charmonium states in $p p$ collisions [86]. The exclusive production of charmonium states in $p p$ interactions is a diffractive process that proceeds through photon and colour singlet (pomeron) exchanges between the intial state protons. Such measurements are useful probes of the very low- $x$ gluon distribution $\left(x \sim 10^{-6}\right)$ in the proton PDF, a region of phase space difficult to access in inelastic $p p$ interactions.

The ATLAS, CMS and LHCb experiments have measured the cross sections for $\Upsilon(n S)$ production at $\sqrt{s}=7 \mathrm{TeV}$ [87-89] and at $\sqrt{s}=8 \mathrm{TeV}$ [80]. The LHCb experiment has measured the fraction of $\Upsilon(1 S)$ produced in radiative $\chi_{b}(1 P)$ decays to be around $20 \%$ and largely independent of $\Upsilon(1 S)$ transverse momentum [90]. The CMS experiment has measured the $\Upsilon(n S)$ polarisation to be slightly transverse, with sequentially stronger transverse polarisation for the $\Upsilon(2 S)$ and $\Upsilon(3 S)$ states [91]. 

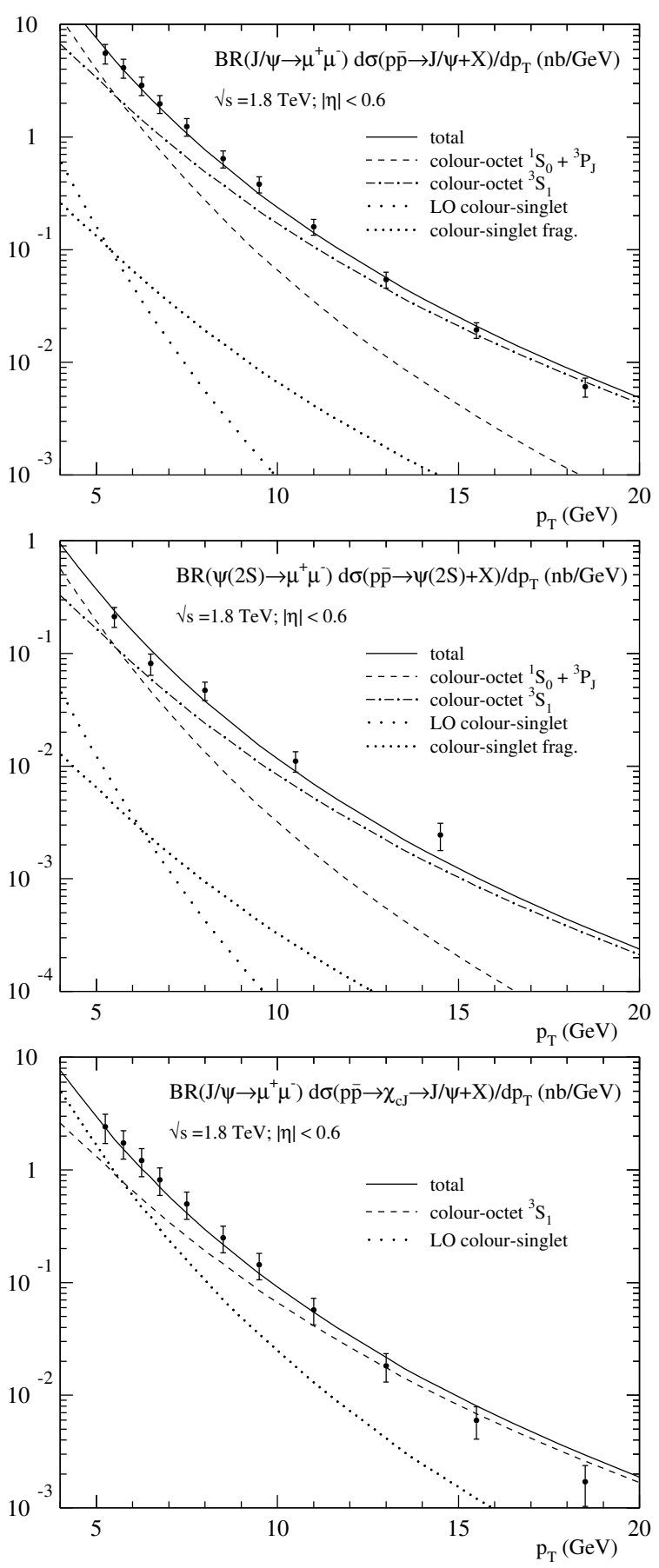

Figure 3.6: CDF measurements of charmonium production in $p \bar{p}$ collisions at $\sqrt{s}=1.8 \mathrm{TeV}$. The upper figure shows the direct $J / \psi$ production cross section, determined by subtracting contributions from $\chi_{c}$ and $\psi(2 S)$ feed-down. The middle figure shows the prompt $\psi(2 S)$ production cross section while the lower figure shows the contribution to the prompt $J / \psi$ production cross section from radiative $\chi_{c}$ decays. The differential cross sections in each figure are compared with the prediction from NRQCD (the sum of all individual curves, denoted total) and the CSM. All figures from Ref. [55]. 


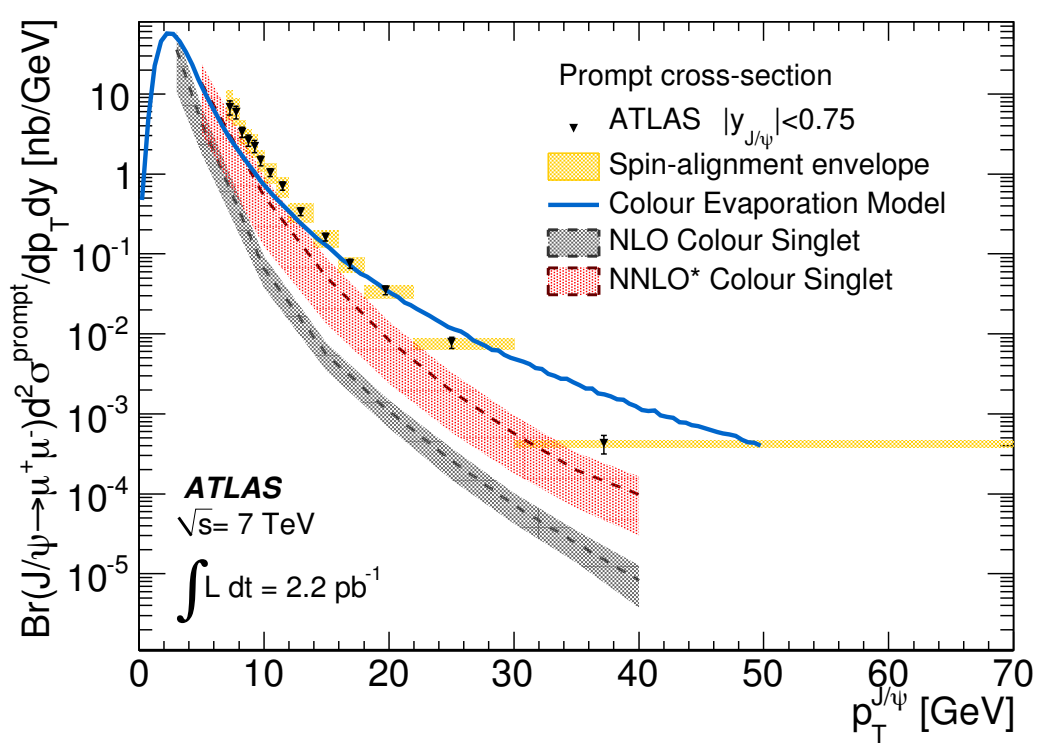

(a)

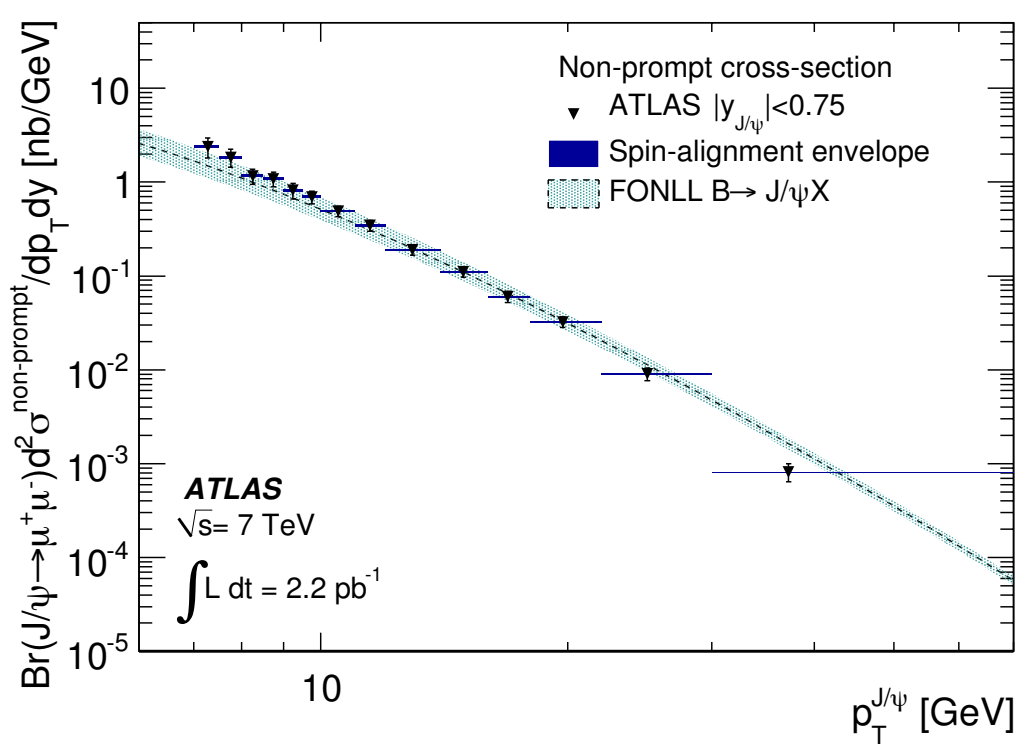

(b)

Figure 3.7: The cross sections for prompt (a) and non-prompt (b) $J / \psi$ production in $p p$ collisions at $\sqrt{s}=7 \mathrm{TeV}$ measured by ATLAS [77]. The measurements of the prompt cross sections are compared to the predictions of the CEM and the CSM while the non-prompt cross section is compared to the predictions of the FONLL approach [56]. 


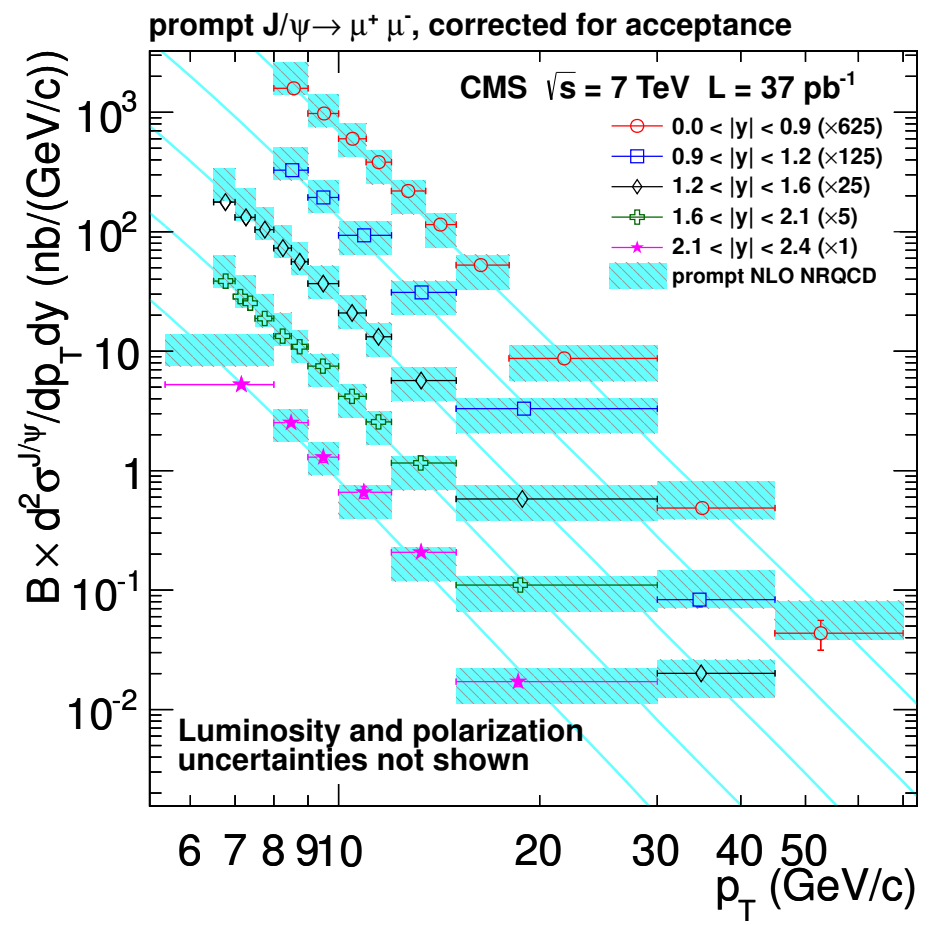

(a)

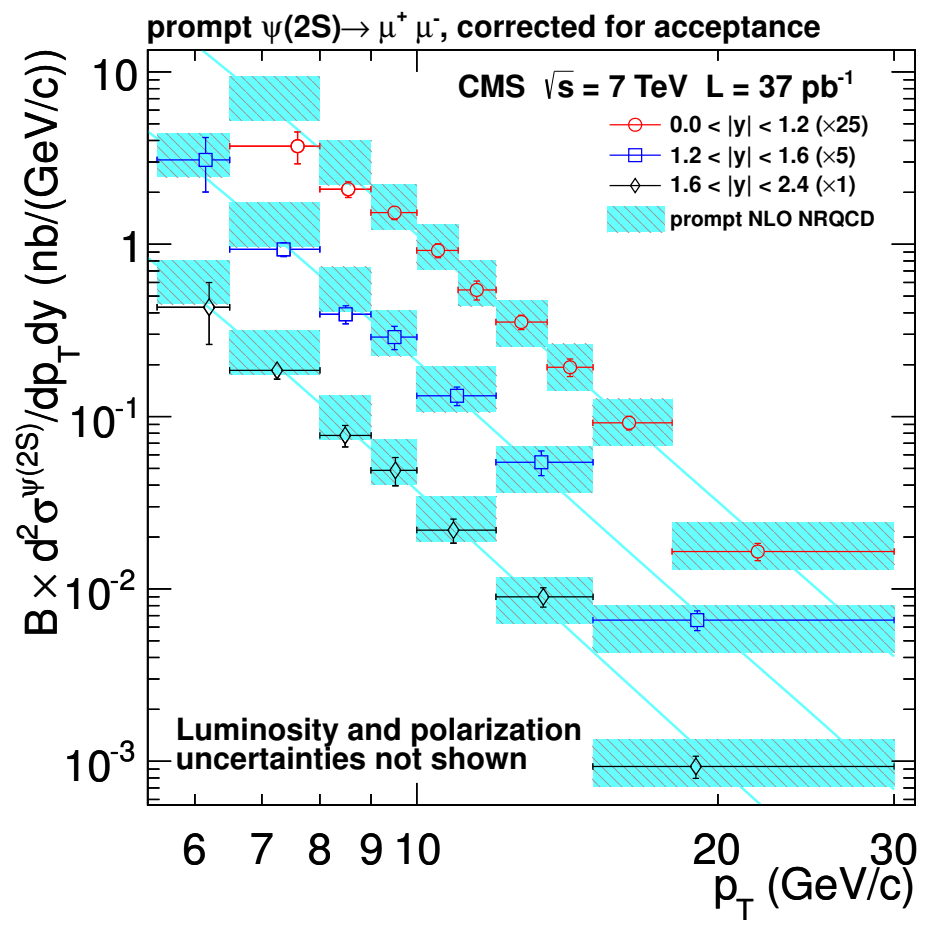

(b)

Figure 3.8: The cross sections for prompt $J / \psi($ a) and $\psi(2 S)$ (b) production in $p p$ collisions at $\sqrt{s}=7 \mathrm{TeV}$ measured by CMS [78]. The measurements are compared to the predictions of NLO NRQCD. 

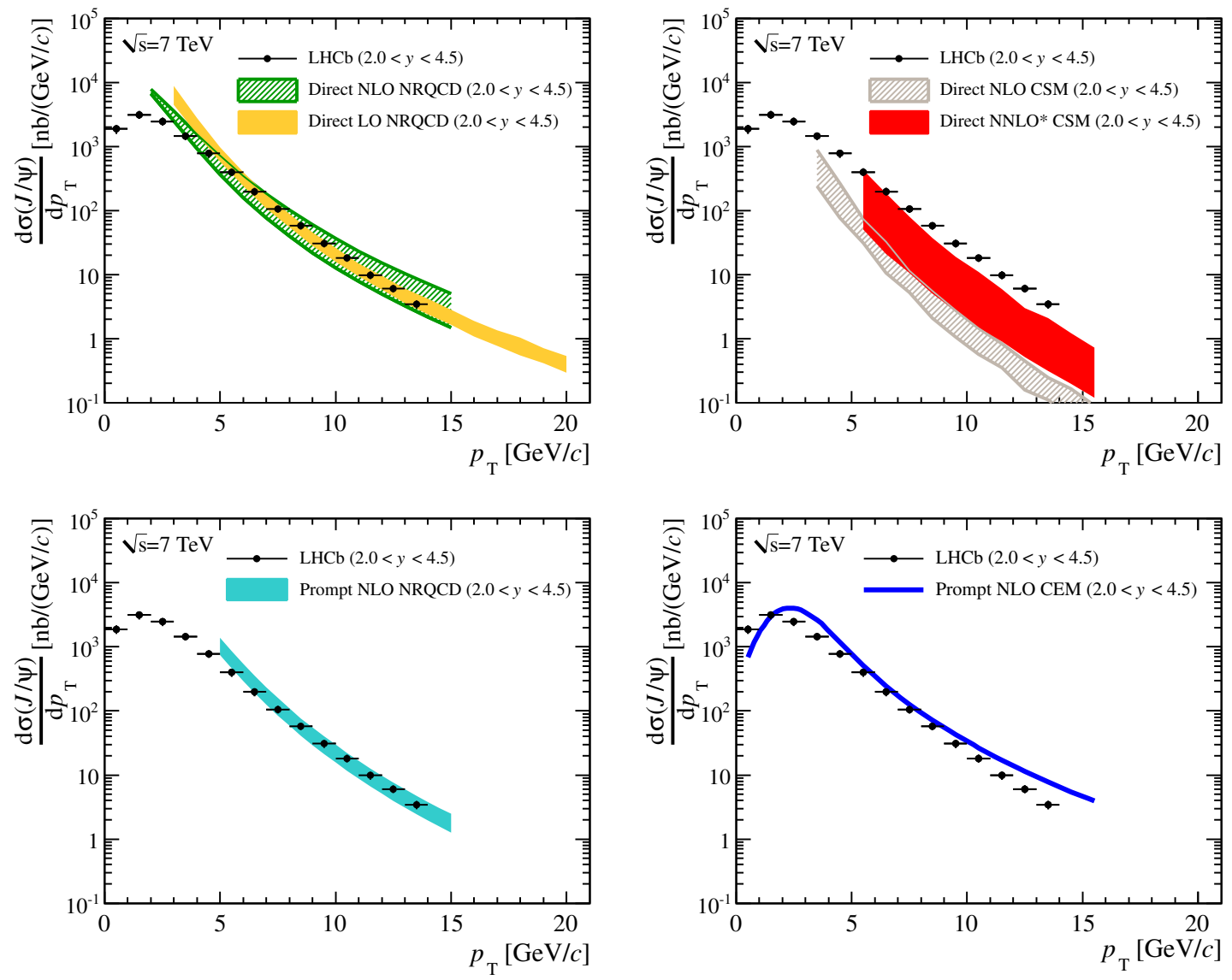

(a)

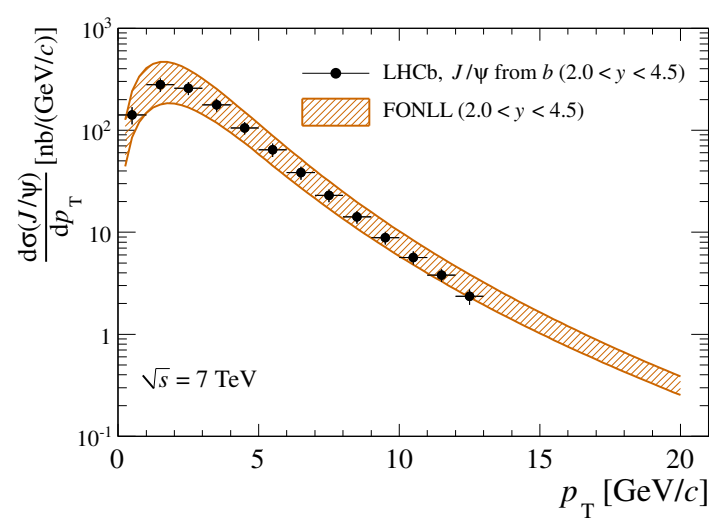

(b)

Figure 3.9: The cross sections for prompt $J / \psi$ production in $p p$ collisions at $\sqrt{s}=$ $7 \mathrm{TeV}$ measured by LHCb [79]. The measurements of the prompt cross sections (a) are compared to the predictions of NRQCD and the CSM while the non-prompt cross section (b) is compared to the predictions of the FONLL approach [56]. 


\section{Chapter 4}

\section{THE ATLAS EXPERIMENT}

The ATLAS experiment (A Toriodal LHC ApparatuS) is designed to study protonproton and heavy ion collisions at the Large Hadron Collider (LHC). The LHC and its experiments were built to study particle physics in hadron collisions at energies significantly higher than had been investigated before. The main motivation of the project was to search for the Higgs boson, candidates for dark matter and other physics beyond the SM.

\subsection{The Large Hadron Collider}

The Large Hadron Collider is a superconducting hadron accelerator and collider situated at CERN near Geneva, Switzerland [92]. The LHC is installed in the $26.7 \mathrm{~km}$ circumference circular tunnel constructed for the Large Electron-Positron Collider (LEP), which operated between 1989 and 2000. The LHC is designed to accelerate two counter-rotating beams of protons in two separate rings up to an energy of $7 \mathrm{TeV}$ per proton and can deliver proton-proton $(p p)$ collisions at a centre-of-mass energy of up to $\sqrt{s}=14 \mathrm{TeV}$. The LHC is also capable of accelerating lead $(\mathrm{Pb})$ ions to deliver both lead-lead and proton-lead collisions. The LHC has four interactions points (IP), shown in Figure 4.1, each instrumented with a modern particle physics experiment. The ATLAS and CMS general purpose experiments are designed for

high-luminosity operation (up to $10^{34} \mathrm{~cm}^{-2} \mathrm{~s}^{-1}$ ) and are equipped to study a wide range of phenomena. Their physics goals include Higgs boson searches (and mea- 
surements), searches for super-symmetric particles and exotic phenomena and measurements of the standard model. Two further dedicated experiments, $\mathrm{LHCb}$ and ALICE, are designed for lower luminosity operation (around $10^{32} \mathrm{~cm}^{-2} \mathrm{~s}^{-1}$ ) and are specially equipped to study $B$-physics and heavy ion collisions, respectively.

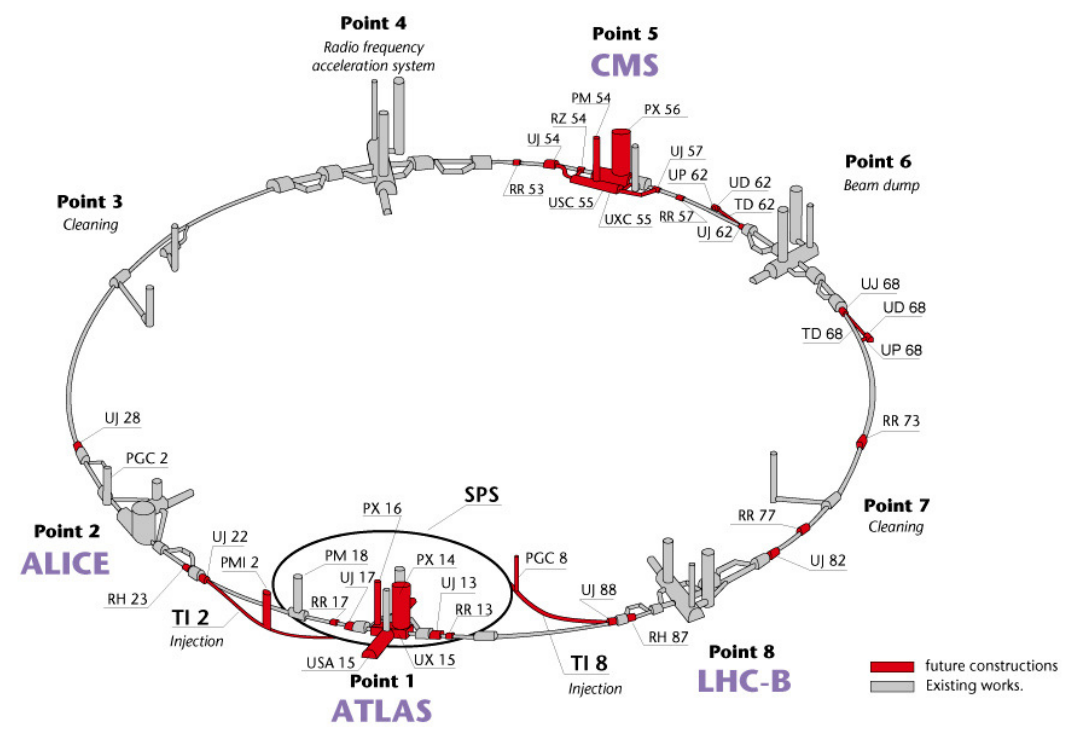

Figure 4.1: A schematic diagram of the LHC ring showing the locations of the four experiments [92]. Image (C) CERN.

Before protons are injected into the LHC, they first experience a multi-stage process of beam preparation, focussing and acceleration within the CERN accelerator complex, as shown in Figure 4.2. First, the linear accelerator Linac2 generates a $50 \mathrm{MeV}$ beam of protons from ionised hydrogen gas. This proton beam is fed into the Proton Synchrotron Booster (PSB), focussed into bunches and accelerated to around 1.4 GeV before being injected into the Proton Synchrotron (PS) ring, which further accelerates the beam to an energy of $26 \mathrm{GeV}$. It is in the PS that proton bunches are arranged into bunch trains. The final stage involves the beam being injected into the Super Proton Synchrotron (SPS) where the beam reaches the LHC injection energy of $450 \mathrm{GeV}$. When operating at its design luminosity, the LHC will be filled with 2808 bunches, each containing around $10^{11}$ protons, with a bunch spacing of $25 \mathrm{~ns}$ to provide a bunch collision rate of $40 \mathrm{MHz}$. 

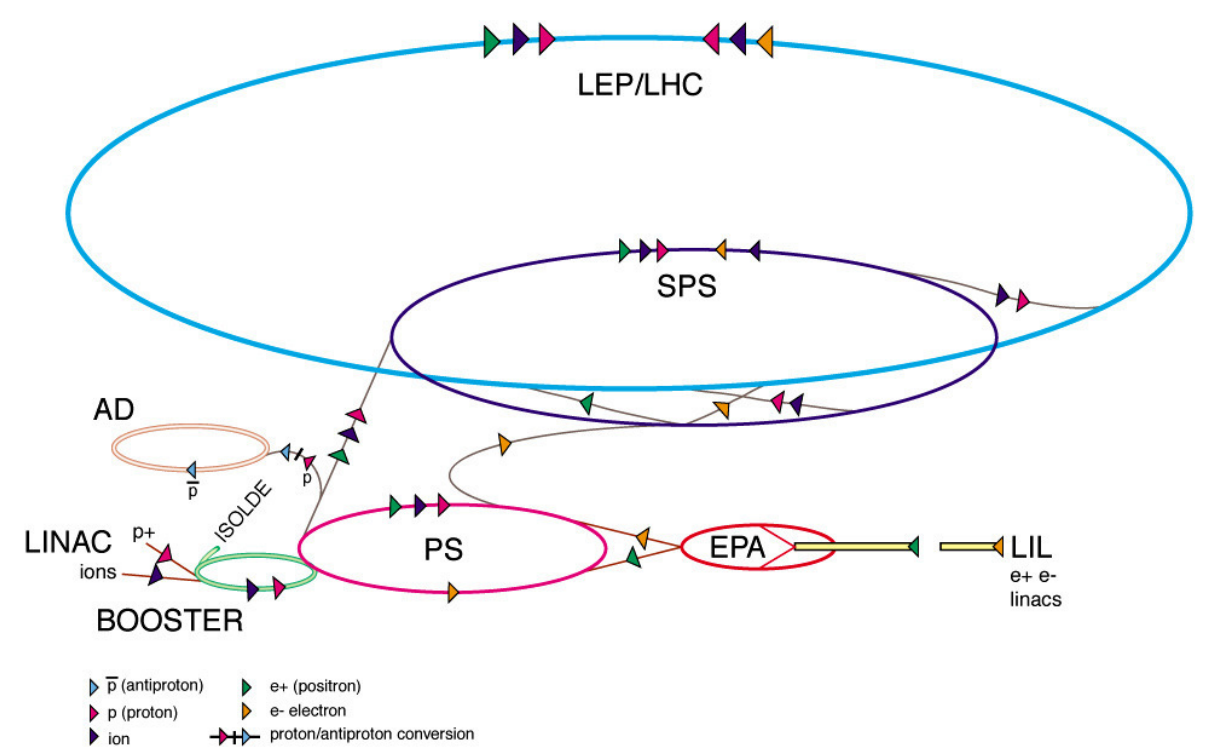

Figure 4.2: The CERN accelerator complex. The particle accelerators involved in the LHC injection chain (LINAC $\rightarrow$ BOOSTER $\rightarrow$ PS $\rightarrow$ SPS $\rightarrow$ LHC) are shown [92]. Image (C) CERN. 


\subsection{Introduction to the ATLAS Detector}

A Toroidal LHC Apparatus (ATLAS) is a general purpose particle physics detector designed to study proton-proton collisions at the TeV scale. The detector is housed in a large cavern, known as interaction point 1, approximately $93 \mathrm{~m}$ below the surface and has a cylindrical geometry, with the direction of the LHC beams defining the axis of symmetry. The detector is composed of several sub-detectors, each designed to detect different kinds of sub-atomic particles. These sub-detectors surround the beam axis in layers and are arranged in a central "barrel" section and two "endcap" sections. Together, these sub-detectors provide almost hermetic coverage that is both forward-backward and axially symmetric. Figure 4.3 shows a computer-generated schematic diagram of the ATLAS detector.

The following description of the ATLAS detector is intended to provide a brief review of the design and operation of ATLAS. This summary is based on the detailed description found in [93].

The ATLAS coordinate system is defined with its origin at the nominal protonproton interaction point (IP). The $z$-axis is defined by the beam direction, with side A of the detector covering $z>0$ (the anti-clockwise direction viewed from above) and side $\mathrm{C}$ of the detector covering $z<0$. The $x$-axis is defined as the direction from the IP to the centre of the LHC ring, while the $y$-axis points vertically upwards, towards the surface. The polar angle $\theta$ is measured in the $r$ - $z$ plane $\left(r=\sqrt{x^{2}+y^{2}}\right)$ as the angle from the positive direction of the $z$ axis. The azimuthal angle $\phi$ is defined in the $x-y$ plane and is measured anti-clockwise around the positive $z$-axis, with $\phi=0$ defined as being along the negative $x$-axis.

The rapidity $y$ (not to be confused with the spatial coordinate $y$ ) is a convenient angular variable for describing massive particles travelling at relativistic speeds,

$$
y=\frac{1}{2} \ln \left[\frac{\left(E+p_{z}\right)}{\left(E-p_{z}\right)}\right],
$$




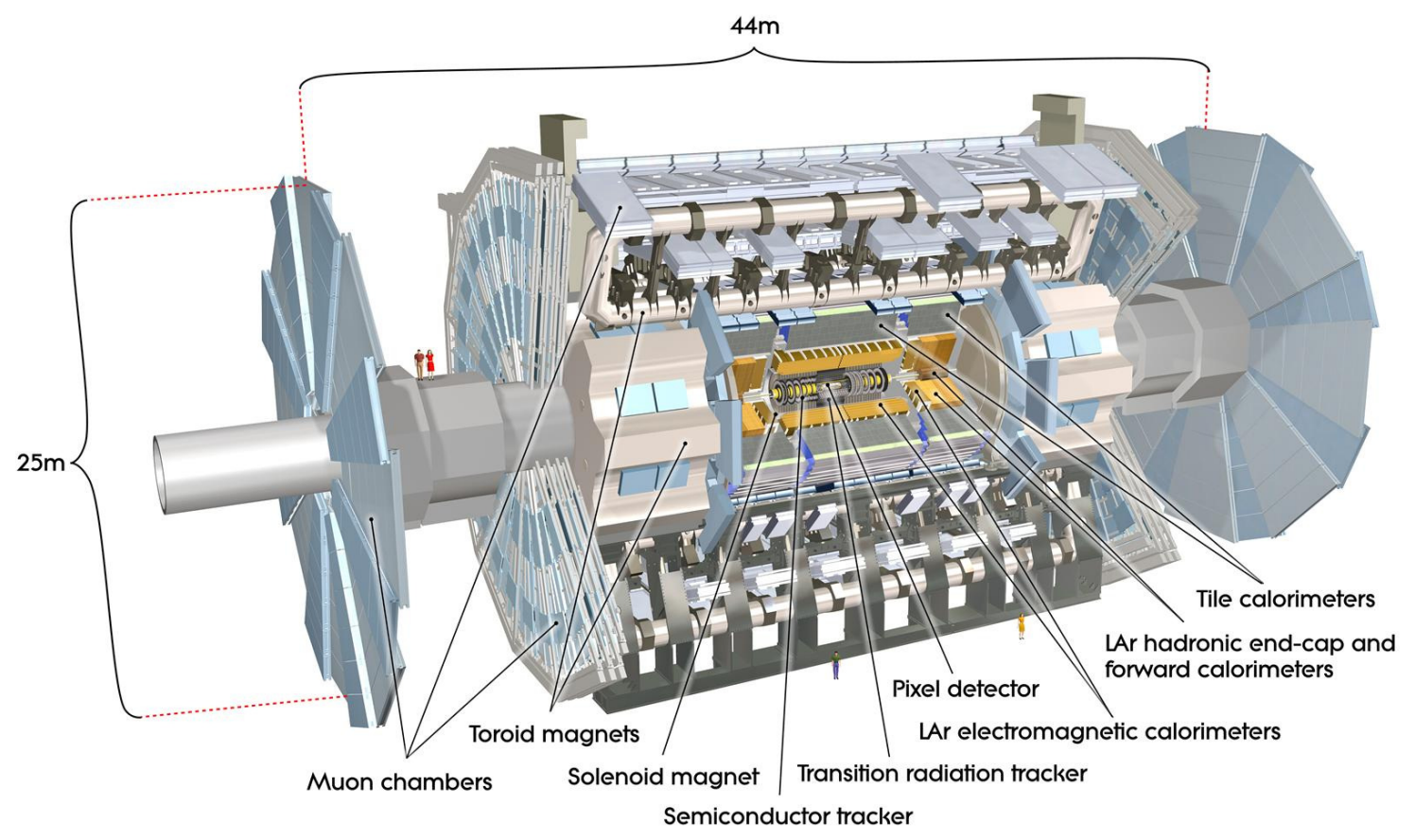

Figure 4.3: A schematic diagram showing a slice through the ATLAS Detector [93]. Image (C) CERN.

where $E=\sqrt{|\vec{p}|^{2}+M^{2}}$ is the energy of a particle of mass $M$ travelling with momentum $\vec{p}$ and $p_{z}$ is the component of $\vec{p}$ in the direction of the beam $(z)$ axis. For massless or highly relativistic $(E \approx|\vec{p}|)$ particles, the pseudorapidity $\eta$ is often used,

$$
\eta=-\ln \left[\tan \left(\frac{\theta}{2}\right)\right]
$$

where $\lim _{M \rightarrow 0} y=\eta$. The transverse momentum $p_{T}$ and transverse energy $E_{\mathrm{T}}$ are defined in the $x-y$ plane with $p_{T}^{2}=p_{x}^{2}+p_{y}^{2}$ and $E_{\mathrm{T}}=E \cdot \sin (\theta)$.

\subsection{Inner Detector}

The ATLAS Inner Detector (ID) is an ensemble of three precision tracking detectors designed to measure the momenta of charged particles produced at the IP and to identify primary and secondary charged particle vertices. The pixel detector consists of three layers of silicon pixel modules and is arranged around the beam pipe. The 
Semiconductor Tracker (SCT) surrounds the pixel detector with four stereo layers of silicon microstrip detectors. The Transition Radiation Tracker (TRT) surrounds the SCT and is composed of many layers of gas-filled drift tubes. These three subdetectors are surrounded by a superconducting solenoidal magnet that immerses the inner detector in a roughly uniform axial magnetic field of $2 \mathrm{~T}$ to facilitate the measurement of charged particle transverse momenta. Together, these detectors provide charged particle tracking that covers the region $|\eta|<2.5$. The relative position of the individual components of the ID are shown in Figure 4.4. The active layers and associated services and support structures are shown in Figure 4.5.

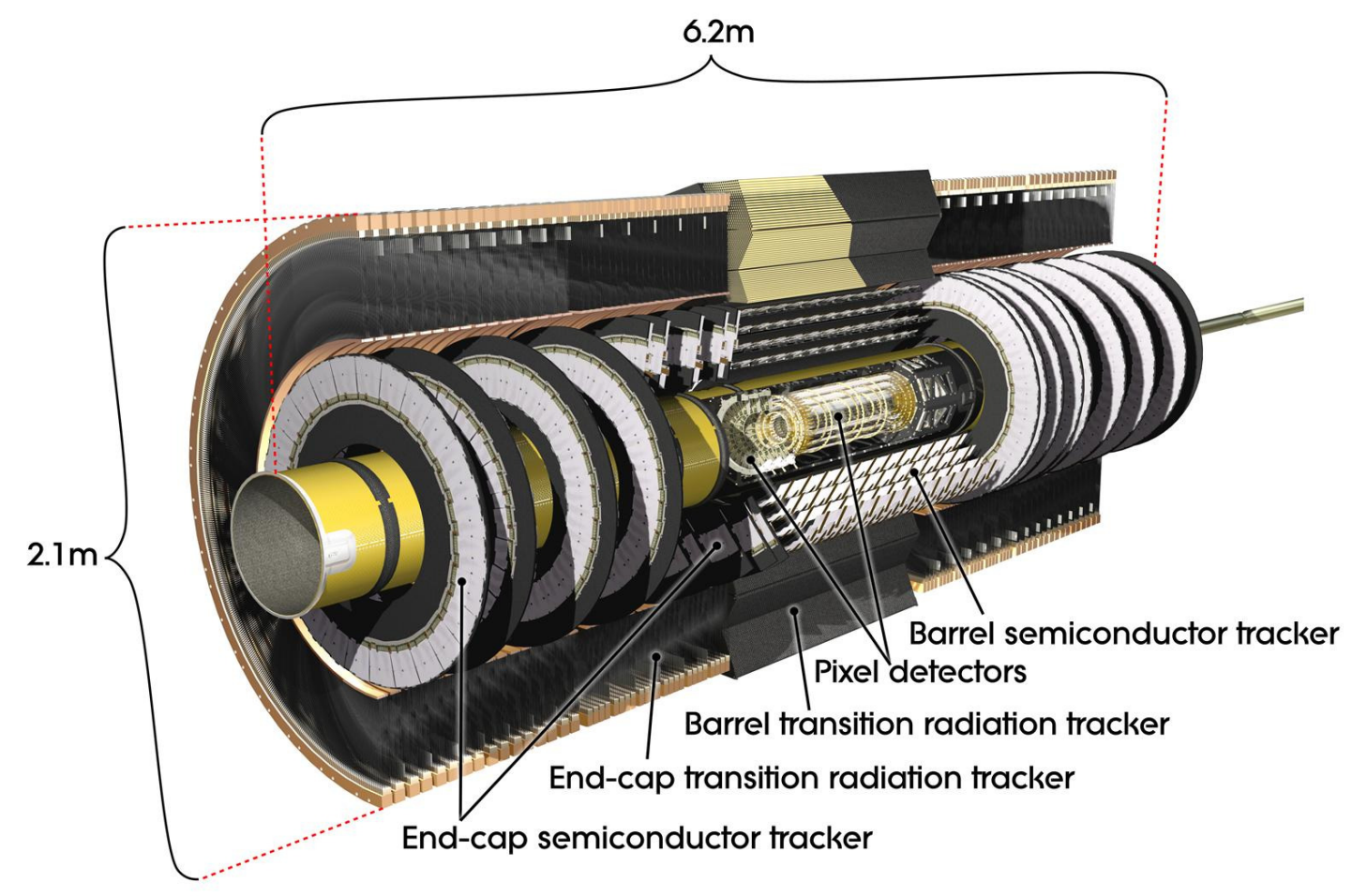

Figure 4.4: A schematic diagram the showing the individual components of the ATLAS Inner Detector (ID) [93]. Image (C) CERN.

\subsubsection{Pixel Detector and Semiconductor Tracker}

The silicon pixel detector is designed to provide the first three space point measurements for the tracks of charged particles produced at the nominal IP. The detector is composed of a central barrel containing three concentric layers of modules and two 


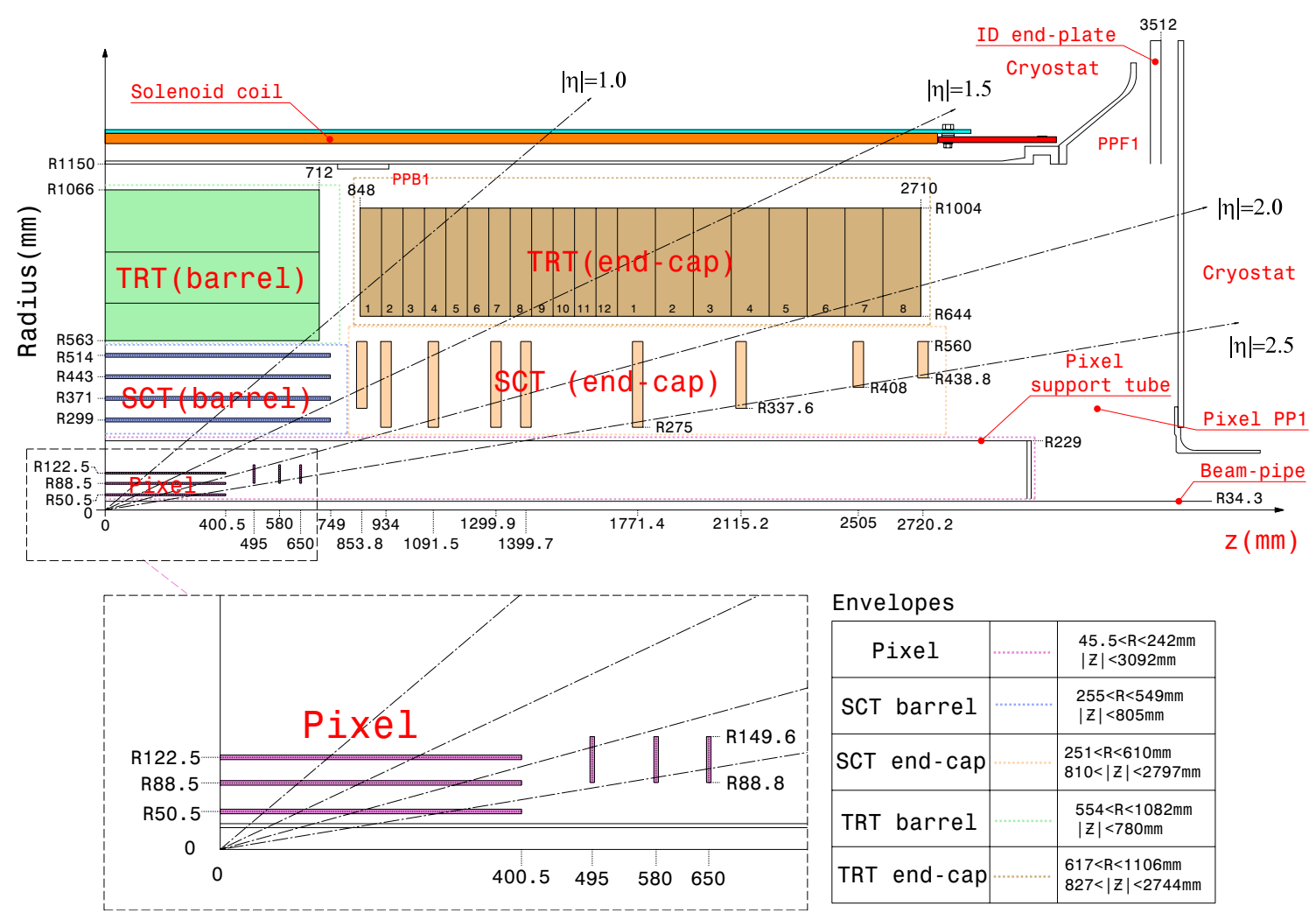

Figure 4.5: A schematic diagram showing an $r-z$ view of the active layers of the ATLAS inner detector in addition to the major services and support structures [93]. Image from Ref. [93].

endcaps, each containing three disks of modules arranged in layers perpendicular to the beam axis, as shown in Figure 4.4. The detector instruments the radial region $50.5<r<150.0 \mathrm{~mm}$ and contains 1744 silicon pixel modules [93]. Each pixel module contains 47232 pixels distributed over an active area of $16.4 \times 60.8 \mathrm{~mm}^{2}$ with the majority of pixels having an area of $50 \times 400 \mu \mathrm{m}^{2}$ [93]. Each pixel module contains 16 radiation-hard front-end chips, which provide read out for the pixel sensors. Hits in the detector are registered and read out if the signal in a given pixel exceeds an adjustable threshold [94]. The pixel layers provide space point measurements with a precision in the $r$ - $\phi$ plane of $10 \mu \mathrm{m}$. The barrel layers also provide measurements with a precision of $115 \mu \mathrm{m}$ in the $z$ direction while the disk layers provide measurement with a precision of $115 \mu \mathrm{m}$ in the $r$ direction [95].

The SCT is composed of 4088 silicon strip modules arranged in a central barrel containing four concentric layers, and two endcaps each containing nine disks 
arranged perpendicular to the beam axis, as shown in Figure 4.4. The detector is designed to provide four space point measurements (from eight strip measurements) for charged particles produced at the nominal IP and instruments the radial region $299<r<560 \mathrm{~mm}$ [93]. The majority of modules contain four strip sensors; two daisy-chained sensors are positioned on either side of each module that together provide 768 strips, each $12 \mathrm{~cm}$ long [94]. The strips on either side of the module are oriented with a stereo angle of $40 \mathrm{mrad}$ between them to provide a single space point measurement [93]. The sensors on each module are read out by 12 radiationhard chips, each responsible for 128 channels. The detector registers a hit if the pulse height exceeds a preset threshold corresponding to a charge of $1 \mathrm{fC}$ [94]. The SCT provides space point measurements with a precision in the $r$ - $\phi$ plane of $17 \mu \mathrm{m}$. The barrel layers provide measurements with a precision of $580 \mu \mathrm{m}$ in the $z$ direction while the disk layers provide measurements with a precision of $580 \mu \mathrm{m}$ in the $r$ direction [95].

The data transfer systems of the pixel and SCT detectors use optical transmission to send trigger, timing and control signals to the modules and to read out hit data from the modules. The optical signals are transmitted by Vertical Cavity Surface Emitting Lasers (VCSELs) operating at a wavelength of $850 \mathrm{~nm}$ [94].

\subsubsection{Transition Radiation Tracker (TRT)}

The TRT is made up of 298304 proportional drift tubes. Each tube is $4 \mathrm{~mm}$ in diameter and is filled with a gas mixture containing $\mathrm{Xe}, \mathrm{CO}_{2}$ and $\mathrm{O}_{2}$ [93]. The TRT is composed of a barrel and two endcaps, as shown in Figure 4.4. The drift tubes in the barrel section are $144 \mathrm{~cm}$ long and arranged in three concentric layers split into 32 uniform sectors in $\phi$. The drift tubes in the endcaps are $37 \mathrm{~cm}$ long and arranged radially in 80 wheels [94]. The TRT instruments the radial region $563<r<1066 \mathrm{~mm}$ and is designed to provide over 30 space point measurements for charged particles with $p_{T}>500 \mathrm{MeV}$ and $|\eta|<2.0$ produced at the nominal IP [93]. 
The TRT tubes are interleaved with polypropylene fibres or foils to provide electron identification through transition radiation measurements. Signals from ionisation electrons and transition radiation photons (which generally have a much higher energy) are detected by separate low and high threshold discriminators in the frontend electronics [94]. Measurements in the TRT provide space point measurements only in the $r-\phi$ plane with a precision of $130 \mu \mathrm{m}$ for each tube.

\subsection{Calorimeter Systems}

The ATLAS detector includes several sampling calorimeter systems (shown in Figure 4.6) designed to measure the energy (and to provide position and direction information) of electrons, photons, $\tau$ leptons and hadron jets.

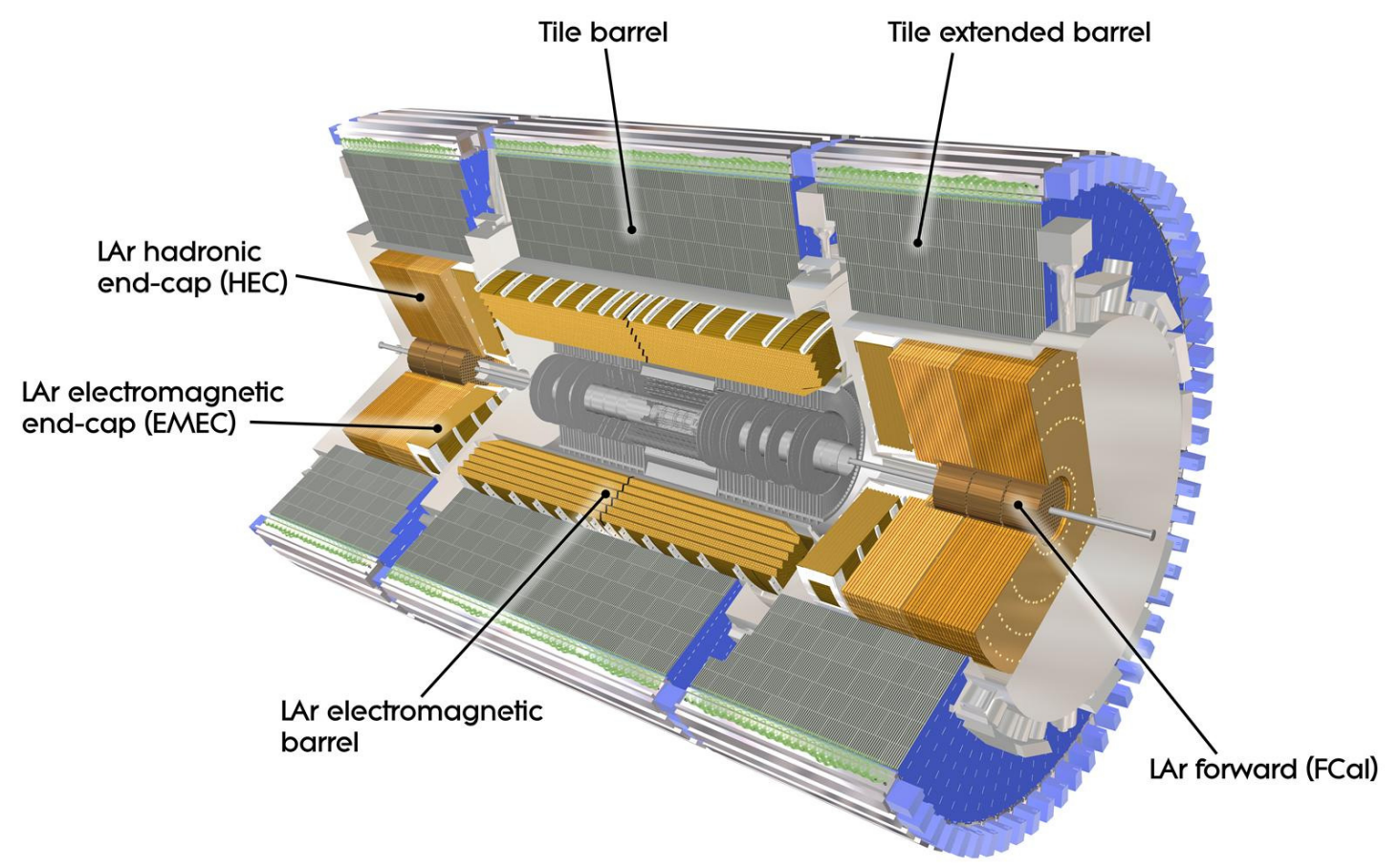

Figure 4.6: A schematic diagram of the ATLAS calorimeter systems [93]. Image (c) CERN. 


\subsubsection{Liquid Argon Calorimeter}

The ATLAS Liquid Argon (LAr) calorimeter is a system of sub-detectors designed for both electromagnetic and hadronic calorimetry. The calorimeter is composed of a central barrel section and two endcaps, with the active sampling detectors housed in three large cryostats [96]. Providing full symmetric coverage in $\phi$, the electromagnetic (EM) calorimeter has a high granularity and is composed of layers of lead absorber and liquid argon instrumented with electrodes in an accordion shaped design. It consists of an EM barrel (EMB) section, which covers the region $|\eta|<1.475$, and two EM end-cap (EMEC) sections, which together cover the region $1.375<|\eta|<3.2$. These three sections are supplemented with a presampler (PS) layer, with a coverage of $|\eta|<1.8$, to provide additional measurements that can be used to correct for particle energy losses in the inner detector, service regions and cryostats. In addition to the LAr EM calorimeters, two LAr hadronic endcap (HEC) calorimeters, using copper absorber are installed behind the two EMEC calorimeters, which together cover the region $1.5<|\eta|<3.2$. In the forward region, $3.1<|\eta|<4.9$, a LAr EM calorimeter with copper/tungsten absorbing layers, known as the FCal, is also installed.

In the EMB, each active calorimeter module is 22 radiation lengths $\left(X_{0}\right)$ deep at $\eta=0$, increasing to $33 X_{0}$ at $|\eta|=1.3$. The EMEC has an active depth of between $24 X_{0}$ to $38 X_{0}$ from $|\eta|=1.475$ to $|\eta|=2.5$. The main EM calorimeters (excluding the PS) are longitudinally segmented into three layers. In the EMB the first layer, known as the strip layer, has the finest granularity in $\eta$ and contains cells with a granularity in $\eta$ and $\phi$ of $\Delta \eta \times \Delta \phi=0.0031 \times 0.098$. The strip layer has a depth of $2.6 X_{0}$ and is designed to provide high resolution direction information. The second layer, known as the middle layer, has a depth of $16 X_{0}$ radiations lengths and is designed to contain the majority of the EM shower. The middle layer has a granularity of $\Delta \eta \times \Delta \phi=0.025 \times 0.0245,4$ times finer in $\phi$ than the strip layer but 


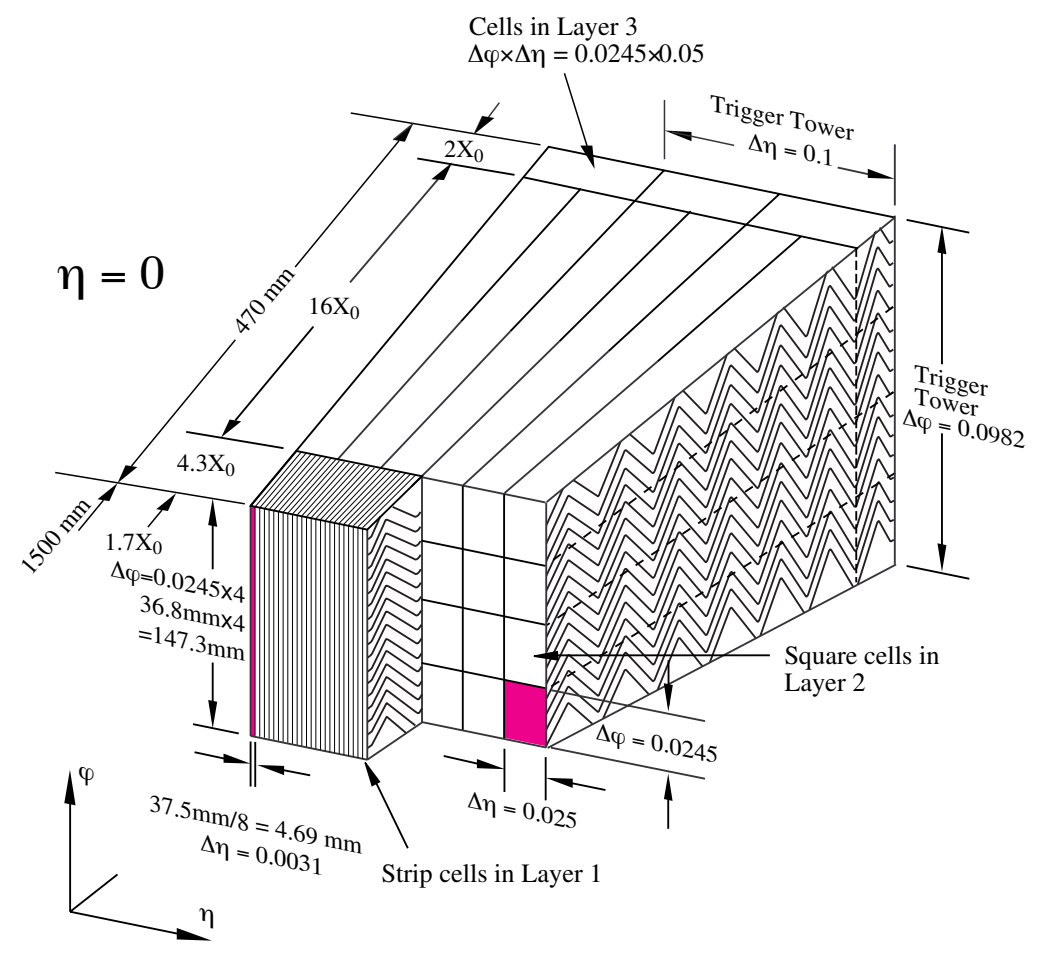

Figure 4.7: A schematic diagram showing a slice through a barrel module of the ATLAS LAr EM calorimeter. Image from Ref. [93].

around 8 times coarser in $\eta$. The final layer, known as the back layer, is present to collect the small residual energy of the shower and has a depth of $2 X_{0}$. The back layer has a coarse granularity with cells of size $\Delta \eta \times \Delta \phi=0.05 \times 0.0245$. This segmentation in $\eta, \phi$ and depth is apparent in Figure 4.7, where a slice through a barrel module of the LAr EM calorimeter is shown. The granularity for the EMEC modules is identical to that of the barrel, except that the back layer has a granularity twice as coarse in $\eta$. This three layer design allows the calorimeter to measure both the energy and direction of the EM shower, improving the overall momentum resolution for reconstructed electrons and photons. In addition to these three main layers, the PS layer, located within the cryostat but in front of the strip layer, has a granularity of $\Delta \eta \times \Delta \phi=0.2 \times 1.52$. The layout of the cells within the HEC calorimeter is shown in Figure 4.8.

The typical energy resolution of the ATLAS LAr calorimeters is given by 


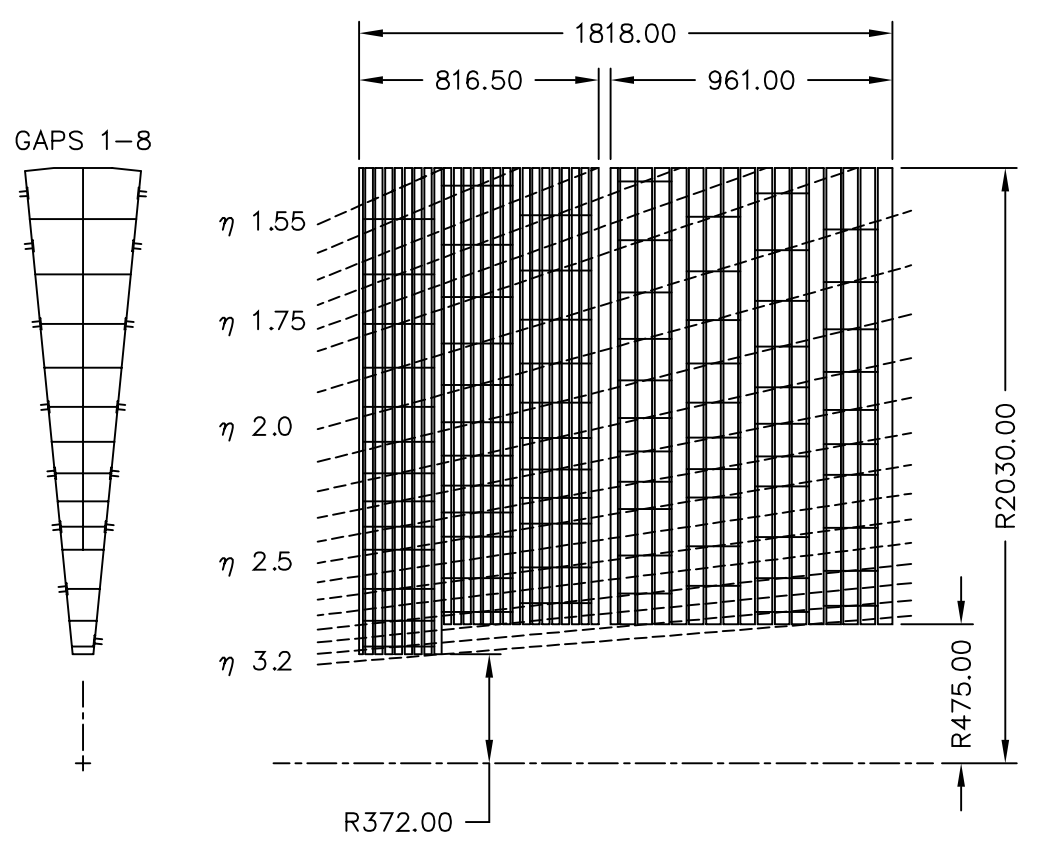

Figure 4.8: A schematic diagram showing the segmentation in $r \times \phi$ (left) and $r \times z$ (right) of the Hadronic Endcap (HEC) calorimeter. Image from Ref. [93].

$$
\frac{\sigma_{E}}{E}=\frac{A}{\sqrt{E}} \oplus \frac{B}{E} \oplus C
$$

where $E$ is measured in units of $\mathrm{GeV}$ and the symbol $\oplus$ denotes addition in quadrature [96]. The terms with coefficients $A, B$ and $C$ are often called the stochastic, noise and constant terms, respectively, with typical values of $A=0.1 \sqrt{\mathrm{GeV}}$, $B=0.17 \mathrm{GeV}$ and $C=7 \times 10^{-3}[96]$.

\subsubsection{Tile Calorimeter}

In addition to the forward hadronic coverage of the ATLAS HEC LAr calorimeter, the Tile Calorimeter provides coverage in the central region of $|\eta|<1.7$. The Tile Calorimeter is located behind the LAr EM calorimeter and consists of steel absorbing layers with tiles of scintillator as the active medium, read out with photomultiplier tubes. The calorimeter is divided into a barrel section, which covers the region $|\eta|<$ 1.0, and two extended barrel sections, which cover the regions $0.8<|\eta|<1.7$. The three sections are each segmented into 64 modules with a granularity $\Delta \phi \sim 0.1$ [97]. 
Each module is longitudinally segmented into three layers. The inner two layers have a granularity in $\eta$ of $\Delta \eta=0.1$ while the third layer has an $\eta$ granularity two times as coarse. The segmentation is shown in the $r-z$ plane is shown in Figure 4.9. The Tile Calorimeter has an energy resolution of approximately $\sigma_{E} / E=50 \% / \sqrt{E(\mathrm{GeV})} \oplus 3 \%$ for hadronic jets [97].

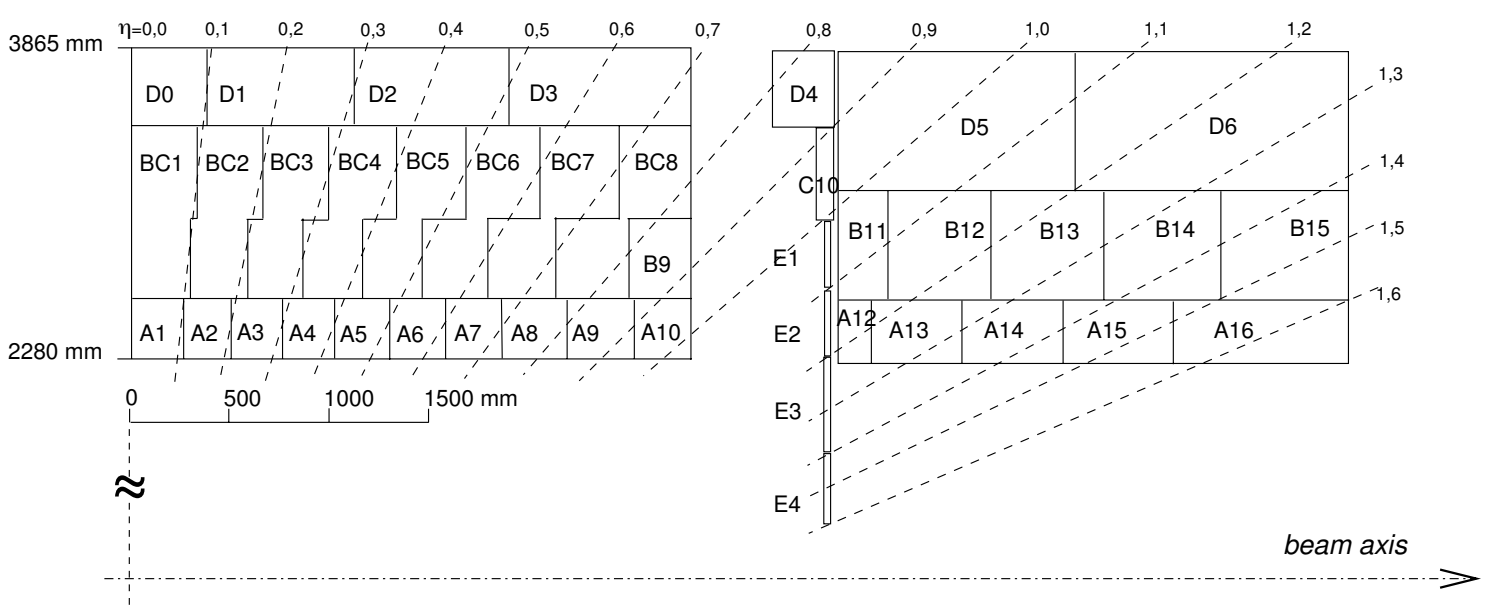

Figure 4.9: A schematic diagram showing the segmentation in $r \times z$ (and $\eta$ in dashed lines) of the Tile Calorimeter. Image from Ref. [93].

\subsection{Muon System}

The muon system is designed to detect charged particles, most commonly muons, that penetrate beyond the calorimeter systems. It consists of a set of large aircore superconducting toroid magnets instrumented with several detector systems, collectively known as the Muon Spectrometer (MS). The MS has coverage across the region $|\eta|<2.7$, and provides independent momentum measurements for penetrating charged particles by tracking particle trajectories within the magnetic field formed by the toroid magnets. It also functions as a trigger detector within the region $|\eta|<2.4$. The MS is arranged in a barrel section and two end-cap sections. The active regions of the MS are the muon chambers, which are arranged in three cylindrical layers in the barrel region, and three layers in the endcaps, arranged in wheels with chambers aligned perpendicular to the beam axis. Correspondingly, the toroid magnet system 
is composed of a central barrel toroid magnet array and two end-cap toroids. The arrangement of the muon chambers is shown in Figure 4.10.

The MS is composed of four different varieties of chambers, two types for tracking and two for triggering, each of which has a different detector design and exploits different technologies. Monitored Drift Tube (MDT) chambers have coverage over the majority of the instrumented region in $\eta$ and provide precision tracking measurements in the bending plane of the toroid magnets. The MDT detectors are supplemented with Cathode Strip Chambers (CSC) in the high $\eta$ region of $2.0<|\eta|<2.7$. The muon trigger detector is composed of Resistive Plate Chambers (RPC) in the barrel region and Thin Gap Chambers (TGC) in the end-cap regions.

\subsubsection{Precision Tracking Detectors}

The tracking chambers are designed to provide a precise momentum measurement for charged particles in the bending plane $(r-z)$ of the toroid magnetic field, which can represent a stand-alone measurement, but can also be complimented with a measurement in the ID. The MDT chambers are composed of two layers of drift tubes, each between 3 or 4 tubes thick, separated by a cavity containing readout electronics and a laser-based alignment monitoring system. The tubes are around $3 \mathrm{~cm}$ in diameter and are filled with a gas mixture containing $93 \% \mathrm{Ar}$ and $7 \% \mathrm{CO}_{2}$ in addition to a small amount of water vapour [98]. In the centre of each tube, a tungsten-rhenium anode wire with a diameter of $50 \mu \mathrm{m}$ is mounted to collect the ionisation electrons produced when a charged particle traverses the tube. In addition to the MDTs, CSC chambers are used as they are capable of more robust operation in the forward regions where there are higher backgrounds [98]. The CSC chambers are multiwire proportional chambers with wires oriented in the radial direction [93]. The charge collected on the anode wires is read out with an array of cathode strips [93]. 

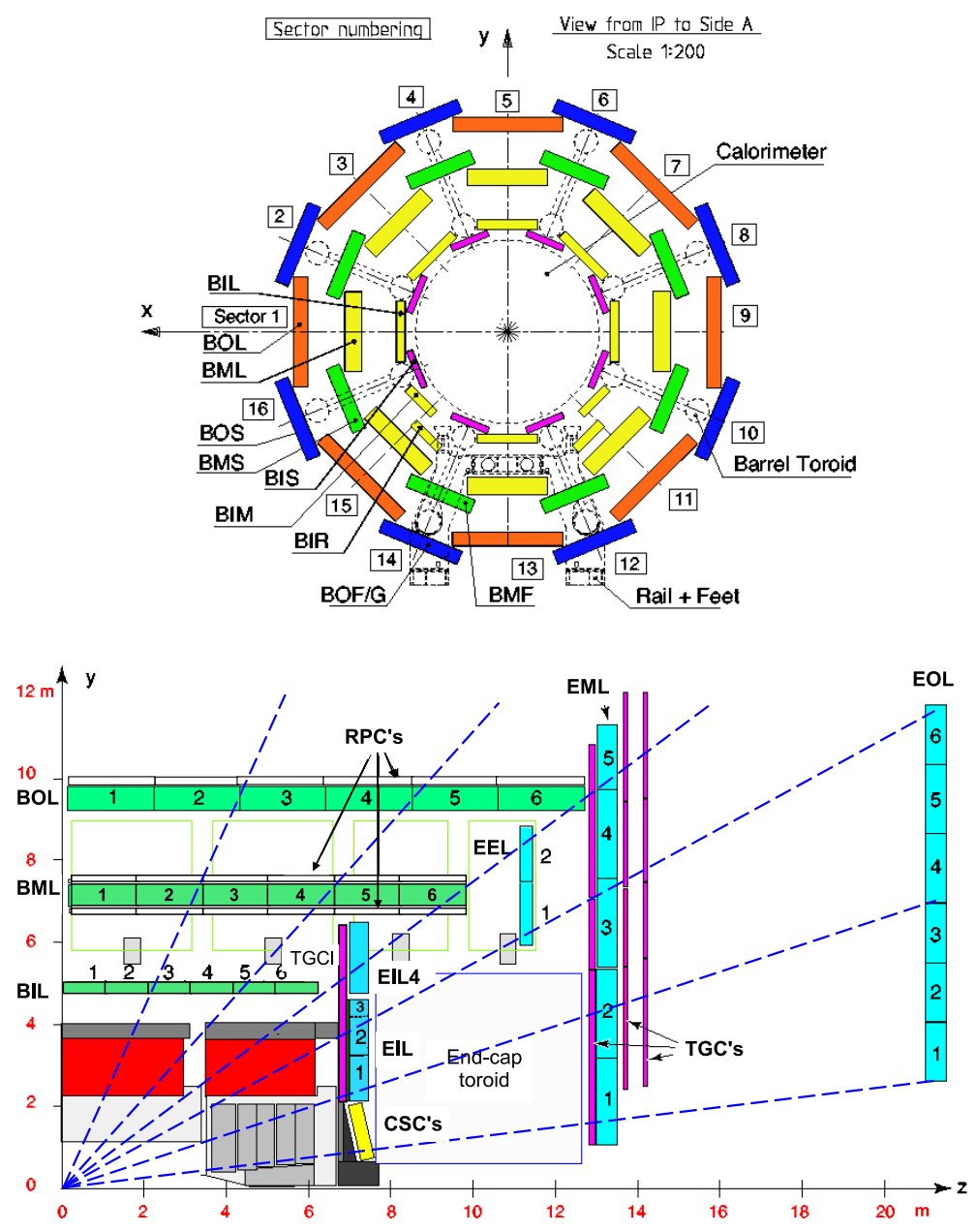

Figure 4.10: A schematic diagram showing a cross section through the ATLAS muon system in both the transverse plane (top) and the $r-z$ bending plane (bottom), showing the arrangement of the different tracking and triggering chambers. Images from Ref. [93].

\subsubsection{Trigger Detectors}

The trigger chambers provide fast measurements of muon position, which can be used to form coincidences to be sent to the first level trigger system. The RPCs installed in the barrel region consist of two parallel electrode plates separated by a distance of $2 \mathrm{~mm}$, with a cavity filled with a gas mixture with a composition dominated by $\mathrm{C}_{2} \mathrm{H}_{2} \mathrm{~F}_{4}$ [93]. An electric field of $4.9 \mathrm{kVmm}^{-1}$ is applied between the two plates to allow an electric discharge towards the anode when an ionising particle crosses. The end cap coverage is provided by TGCs, where an alternative to RPCs 
is required to cope with the increased background rate. The TGCs are essentially multi-wire proportional chambers that use a $\mathrm{CO}_{2}$ and n-pentane gas mixture [93].

\subsection{Data Acquisition and Trigger System}

The ATLAS trigger system is an important component of the experiment that is necessary to allow the efficient and effective performance of the detector given the limited readout rates and data storage capabilities. The nominal LHC $p p$ bunch crossing rate of $40 \mathrm{MHz}$ is too high (given current technologies) for every event to be read out in full and recorded. The trigger system is designed to use a limited amount of coarse granularity detector hit information from the calorimeters and the muon detectors (which can be read out quickly) to characterise the gross features of each event. The system then makes a fast decision, based on pre-defined criteria, on whether to record the event, such that the overall data-taking rate is reduced to an acceptable level of around $100 \mathrm{~Hz}$. The ATLAS trigger system consists of three levels; Level 1 (L1), Level 2 (L2) and Event Filter (EF). Each trigger level sequentially reduces the data rate, using more information and increasingly precise measurements at each level to make gradually more refined decisions.

The first level, L1, reduces the data-rate from the raw bunch crossing rate to around $75 \mathrm{kHz}$ before being passed to L2. Data are stored in pipelines within the on-detector readout electronics for up to $2.5 \mu \mathrm{s}$ (the maximum L1 latency) before the data are either discarded or accepted by an L1 trigger decision. Around $1 \mu$ s of the L1 latency is accounted for by signal propagation delays, leaving the L1 trigger around $1 \mu$ s to make a decision (with $0.5 \mu$ s contingency). The L1 trigger is designed to search for events with high transverse momentum electrons, photons, muons, $\tau$ leptons or hadron jets or large amounts of missing and total transverse energy. Two L1 sub-systems, L1Calo (using calorimeter information) and L1 muon (using MS information) are implemented in custom made electronics to exploit coarse granu- 
larity information to identify these signatures. Up to 256 individual signatures (e.g. muons with several $p_{T}$ thresholds), known as trigger items, can be pre-defined in the L1 central trigger processor (CTP) [93]. The acceptance rate for each item can be independently controlled through the application of a pre-scale factor, so that only a subset of the events that pass the requirements of each trigger item are passed on to L2. In addition to providing the first trigger decision, the L1 trigger identifies regions of interest (ROI), which represent areas of detector activity consistent with the particular trigger signature being searched for. Events accepted by L1 are passed to the L2 trigger, which uses these ROIs as a seed for the L2 trigger algorithms. The L2 and EF triggers together represent the high level trigger (HLT), which is implemented in custom software running on commercial computer hardware. The HLT makes use of full granularity information from the MS and calorimeters and additionally the ID. The L2 trigger uses this information to reduce the event rate to below $3.5 \mathrm{kHz}$, after which events passing the L2 requirements are fully reconstructed in the Event Filter. The full event information is used by the EF to perform the final decision within around $4 \mathrm{~s}$. This allows the calculation of more complicated quantities (such as missing transverse energy) and the use of more sophisticated analysis procedures (such as track vertex fits). The algorithms implemented in the L1 muon trigger and in the HLT that are used to trigger upon the quarkonium decays $\mathcal{Q} \rightarrow \mu^{+} \mu^{-}$are described in Section 5.5.

Events selected by the EF level of the trigger are passed to the EF output nodes known as SFOs. These nodes control the movement of the data from the ATLAS DAQ system to permanent storage at the CERN data recording centre. The SFOs are equipped with a storage capacity that allows data to be buffered for up to 24 hours before transfer to the CERN data recording centre for further processing and permanent storage (though the typical buffer time is much shorter) [93]. At this stage, the data are arranged into individual streams based upon the triggers which fired the event. The computing infrastructure necessary to process and store all of 
the data recorded by the LHC experiments is, in general, far too large for a single institute to manage alone. Instead, a distributed network of computing infrastructure known as the Worldwide LHC Computing Grid (WLCG), often simply referred to as the Grid, was set up to cope with the huge volume of data recorded by the LHC experiments. The Grid is arranged in a hierarchical structure of at least three "Teirs". The CERN data centre represents the first level, "Teir 0", and is responsible for the initial processing and storage of the raw data. The processed data is then shared amongst several "Teir 1" sites (roughly one per participating country, typically housed at national laboratories). "Teir 2" sites (roughly one per participating institute) hold copies of the processed and provide a large scale distributed data analysis facility. 


\subsection{Simulation}

Monte Carlo (MC) simulations play an important role in allowing the data collected by the ATLAS experiment to be exploited to perform physics measurements. These simulations can be split into two distinct stages: the simulation of particle production in $p p$ collisions and the simulation of the response of the ATLAS detector to these particles. While the simulation methods and physics of these two processes are generally distinct, they are directly linked in their application to facilitate the analysis and interpretation of ATLAS data.

The simulation of particle production in $p p$ collisions is generally performed by dedicated computer programs known as event generators. These are typically written by the theoretical particle physics community and are not specific to ATLAS. Two examples of general-purpose event generators which are used by all of the LHC experiments are PYTHIA [99] and HERWIG [100]. Event generators such as these simulate both the hard interaction in a $p p$ interaction and the fragmentation, hadronisation and decays that follow the hard interaction. These programs simulate the $p p$ collision and subsequent processes up to the point where only particles with long proper lifetimes $(c \tau>10 \mathrm{~mm})$ remain [101]. At this point, these "stable" final state particles are passed to the ATLAS detector simulation. Many different event generators are used by ATLAS for the simulation of specific processes; the details of those relevant to quarkonium production measurements will be discussed in Chapter 7.

The ATLAS detector simulation represents a detailed and complete model that can be used to study the response of the detector to the particles produced in $p p$ collisions. It is based upon the GEANT4 simulation framework [101,102]. The core of the simulation is a detailed model of the physical detector, often known as the detector geometry. The nominal geometry is built from detailed construction plans and measurements, detector alignments and known faults. Alternative "distorted" 
geometries, that include additional detector material or intentional misalignments, for example, are also used for studying systematic uncertainties. In addition to the physical detector, the trigger and data acquisition systems are also fully simulated. The program propagates each of the "stable" particles produced by the event generator through the detector model, simulating all interactions with the material of the detector (e.g. particle showers, bremsstrahlung, photon conversions etc.). Energy deposits in the active regions of the detector are recorded in a "hit" file [101]. These hits are then digitised to simulate the electrical response of the detector to particle energy deposits. At this stage, hits from simulated minimum-bias $p p$ interactions can be added to the event to simulate the many individual $p p$ interactions often observed in a real $p p$ bunch crossing in data. Realistic representations of electrical noise and other backgrounds from the LHC beam and the detector cavern can also be added. These simulated electrical signals are then read into the same raw data format recorded by the detector in real data taking. The raw data files can be processed with the same offline reconstruction algorithms used to process the real data. This chain of processing eventually results in simulation samples in the same format used to store the real data intended for physics analysis, allowing data and simulated samples to be studied together within the same physics analysis software framework. 


\subsection{Operation during LHC Run I}

The LHC had its first period of sustained running from 2010 to 2012 after its first collisions in 2009. During this period, known as LHC Run I, the LHC operated mainly at a centre-of-mass energies of $\sqrt{s}=7 \mathrm{TeV}(2010$ and 2011) and $\sqrt{s}=$ $8 \mathrm{TeV}$ (2012). The $2010 \mathrm{LHC}$ run was largely devoted to the commissioning of the accelerator and experiments with the peak instantaneous luminosity reaching $2.1 \times 10^{32} \mathrm{~cm}^{-2} \mathrm{~s}^{-1}$. The $2011 \mathrm{LHC}$ run represented the first prolonged period of high-luminosity running. The instantaneous luminosity reached a peak of $3.7 \times$ $10^{33} \mathrm{~cm}^{-2} \mathrm{~s}^{-1}$, allowing the ATLAS experiment to collect a dataset with an integrated luminosity of over $5 \mathrm{fb}^{-1}$ at $\sqrt{s}=7 \mathrm{TeV}$. The $2012 \mathrm{LHC}$ run, at an increased centre-of-mass energy of $\sqrt{s}=8 \mathrm{TeV}$, delivered a peak instantaneous luminosity of $7 \times 10^{33} \mathrm{~cm}^{-2} \mathrm{~s}^{-1}$, allowing ATLAS to record a dataset with an integrated luminosity of over $21 \mathrm{fb}^{-1}$. The evolution in time of the ATLAS data-taking throughout the 2011 and 2012 runs is shown in Figure 4.11.

The LHC operated with a bunch spacing of $50 \mathrm{~ns}$ during Run I, double the design figure of $25 \mathrm{~ns}$. The high instantaneous luminosities delivered by the LHC during 2011 and 2012 were largely achieved by increasing the intensity close to the ultimate limit of $1.7 \times 10^{11}$ protons per bunch to compensate for the larger bunch spacing. These large bunch intensities resulted in a significant increase in the average number of individual $p p$ interactions taking place in a single bunch crossing. The interactions of multiple $p p$ pairs in a single bunch crossing is an effect known as pileup and is undesirable from an experimental perspective, because it presents many challenges for the event reconstruction and analysis algorithms. The average number of $p p$ interactions per bunch crossing in the 2011 and 2012 ATLAS datasets is shown in Figure 4.12. The mean number is around 9 in the 2011 dataset and around 21 in the 2012 dataset. This level of pileup was not initially planned for the early stages of the LHC programme and required significant modifications to the 
offline reconstruction algorithms. The rapid increases in instantaneous luminosity throughout the running period also required the trigger selections to be periodically modified to accommodate the increased event rates.

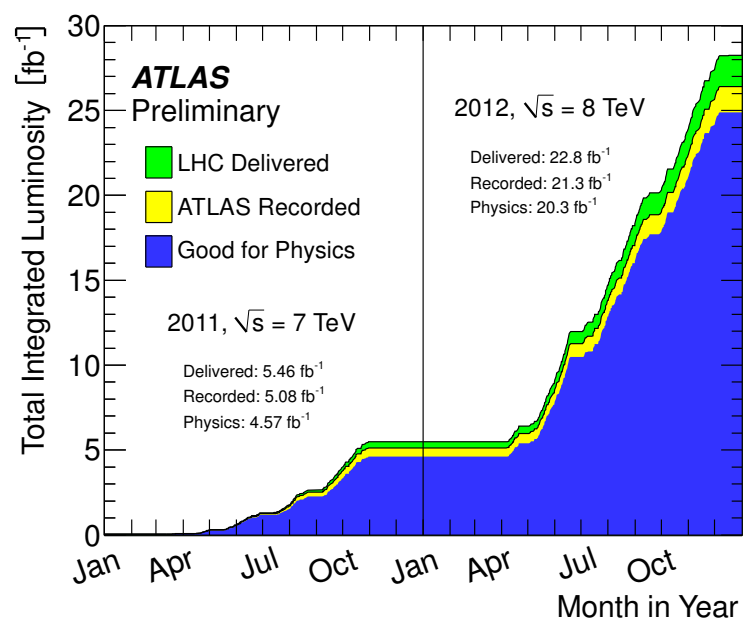

Figure 4.11: The integrated luminosity collected by the ATLAS experiment as a function of time for the 2011 and 2012 LHC runs. Image from Ref. [103].

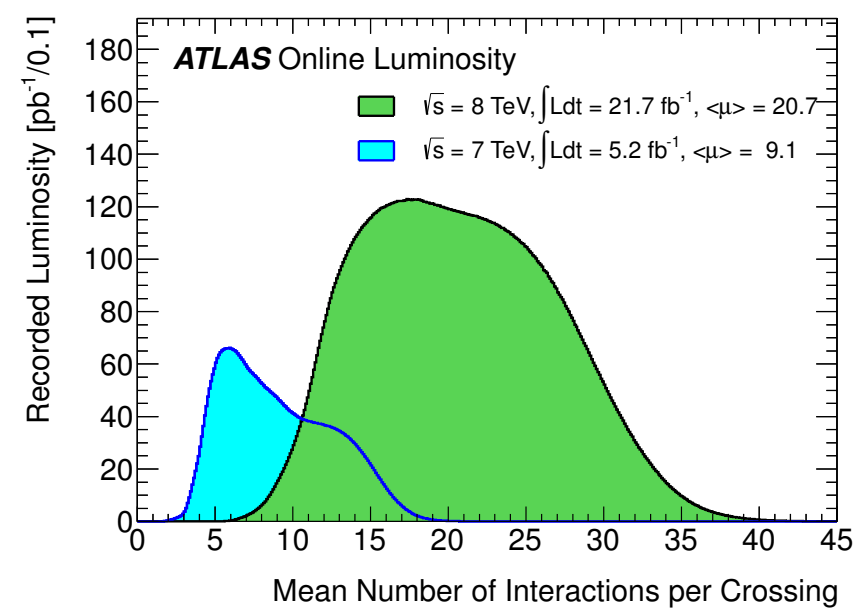

Figure 4.12: The distribution of the average number of $p p$ interactions per bunch crossing $(<\mu>)$ measured during the 2011 and 2012 LHC runs. Image from Ref. [103]. 


\section{Chapter 5}

\section{ANALYSIS TECHNIQUES}

The following sections will discuss a number of experimental data analysis techniques and methods that are exploited to perform the measurements involving the reconstruction of the quarkonium decays $\chi_{b J}(n P) \rightarrow \Upsilon(m S) \gamma$ and $\chi_{c J} \rightarrow J / \psi \gamma$, as described in Chapters 6 and 7 .

\subsection{Charged Particle (Track) Reconstruction}

The ATLAS Inner Detector (tracking detector) is designed to identify and reconstruct charged particles with high efficiency and precision within the region $|\eta|<2.5$. The technical details of the ATLAS ID and its individual tracking sub-detectors are described in Section 4.3. The only charged particles produced in a $p p$ interaction (or through subsequent decay chains) that typically reach the tracking detectors are electrons and positrons $\left(e^{ \pm}\right)$, muons $\left(\mu^{ \pm}\right)$, charged pions $\left(\pi^{ \pm}\right)$, charged kaons $\left(K^{ \pm}\right)$ and (anti-)protons $(p)$. All other charged leptons and hadrons typically decay before traversing the beam pipe. The ATLAS ID is designed to identify and reconstruct all of these charged particle species, albeit with differing efficiency and precision, due to their different interaction characteristics with the material of the detector. The path of a charged particle can be found by measuring its position at several stages in its trajectory as it moves under the influence of the magnetic field produced by the superconducting solenoid magnet. The helical trajectory that a charged particle follows in a uniform magnetic field is exploited to measure its momentum. 
The identification and reconstruction of charged particles within the ATLAS ID proceed in several stages. First, groups of adjacent hits in the pixel detector and silicon strips in the SCT are combined into hit clusters [94]. Hit clusters in the pixel detectors provide direct 3-dimensional space point information, though strip clusters in any given SCT layer only provide a 1-dimensional measurement. Silicon strip clusters on either side of an SCT module are combined with the known input of the position of the module and the stereo angle between the two layers within a module to form 3-dimensional space points [94]. Drift circles in the TRT, representing the radial displacement of a charged particle trajectory from the wire within an individual tube, are reconstructed from knowledge of the relationship between the track-to-wire distance (radius) and the drift time of the ionisation. These can be combined with the 3-dimensional space point information from the pixel and SCT detectors to form an input to a track finding algorithm.

Track reconstruction algorithms use hit information to measure the geometrical parameters of tracks produced by charged particles, which can in turn provide a measurement of the momentum of the particle at a given production point. Several different track finding strategies can be employed for different applications (e.g. prompt tracks from $p p$ collisions, cosmic rays, displaced vertices, etc.). The default track reconstruction procedure first searches for proto-tracks from space point combinations from the three pixel layers and the innermost SCT layer, which are subsequently propagated through the remaining SCT layers to form track candidates [95]. These candidate tracks are then processed by a track fitter algorithm (either a global $\chi^{2}$ minimisation or the Kalman filter technique [104]) and their quality refined through the application of various cuts based on the number of clusters used and dead sensors traversed. These cuts serve to improve the track parameter determination by removing outlying clusters from the track fit and rejecting "fake" track candidates [95]. These silicon-only tracks are then propagated forwards into the TRT, where they are associated with TRT drift circles and the track is refit- 
ted, exploiting information from all three ID sub-detectors. Finally, a comparison between the refitted and silicon-only track is performed, any outlying TRT drift circles are removed from the ensemble of hit measurements and the track is again refitted [95]. Once this procedure has been performed, TRT track segments that are not associated with any reconstructed track are propagated back to the silicon detectors to search for compatible hits to form further track candidates. This method is known as back-tracking and can improve the reconstruction efficiency for tracks that did not originate from the beam axis (e.g. $e^{+} e^{-}$from photon conversions or charged hadrons from $K_{\mathrm{S}}^{0}$ and $\Lambda^{0}$ decays) [95]. The final stage of the process employs an algorithm to search for track vertices in the region of the $p p$ interaction point, known as primary vertices. Further dedicated algorithms also search for track vertices displaced from the beam line to reconstruct photon conversions and $V^{0}$ decays $\left(K_{\mathrm{S}}^{0}, \Lambda^{0}\right.$ and $\left.\bar{\Lambda}^{0}\right)$.

The track finding algorithm determines a set of parameters, typically defined at perigee, the point of closest approach of the track to the $z$-axis of the experiment. In ATLAS, these perigee parameters are shown in Figure 5.1 and are defined as:

$\phi_{0}$ : The angle of the track trajectory in the transverse $(x-y)$ plane at the perigee point

$\theta_{0}$ : The angle of the track trajectory in the $r-z$ plane at the perigee point

$d_{0}$ : The signed impact parameter in the transverse plane, i.e. the distance between the perigee point and the $z$-axis

$z_{0}$ : The distance in $z$ between the perigee point and the origin

$q / p$ : The signed charge of the particle divided by the magnitude of its momentum

These parameters can then be used to determine the four-momentum of the particle that formed the track, with an assumption on the particle's mass. The mass of the particle is chosen either based on the physics application (i.e. the muon mass is 


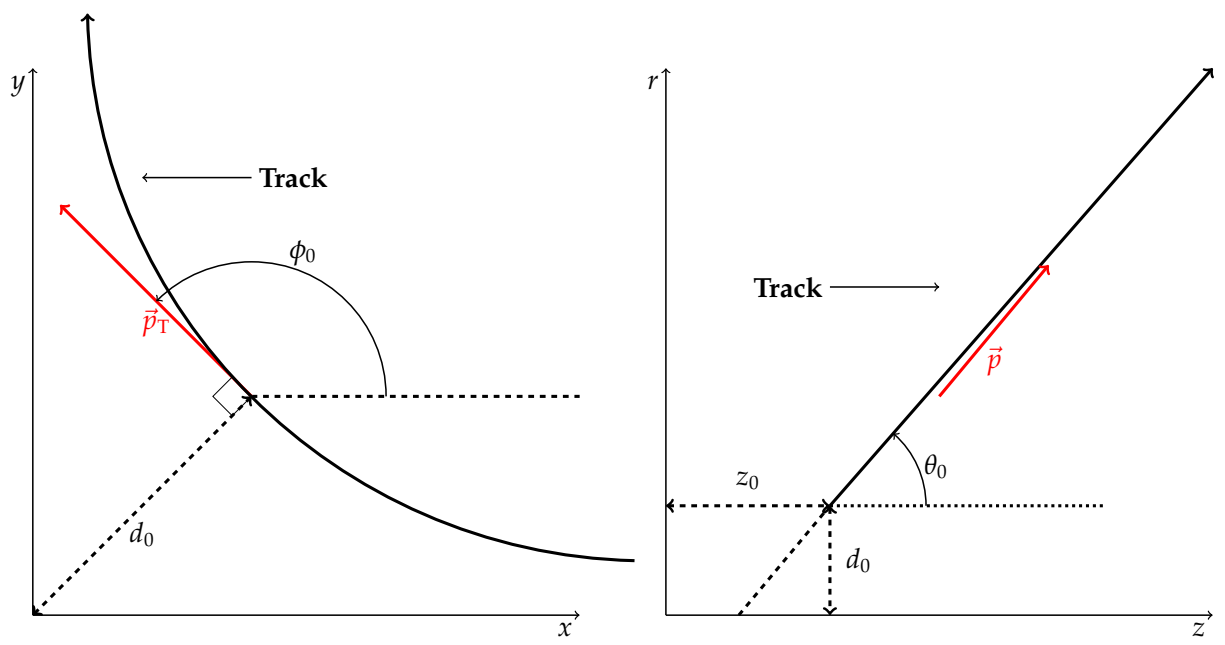

Figure 5.1: Diagram showing the definition of the perigee track parameters in the ATLAS coordinate system in the transverse plane (left) and $r-z$ plane (right).

chosen for $J / \psi \rightarrow \mu^{+} \mu^{-}$studies with ID tracks) or the limited particle identification capabilities of the ATLAS detector can be used to discriminate between the various mass hypotheses. Transition radiation information in the TRT can be used to form an electron probability discriminant for tracks with sufficiency high transverse momentum. Additionally, the rate of energy loss, $d E / d x$, in the pixel layers can be used to provide some information to identify which particle species formed a track, but this is only effective for very low $p_{T}$ tracks (less than $1.5 \mathrm{GeV}$ ). The typical resolution on the track parameters are shown in Figure 5.2 as a function of track $|\eta|$. The relative resolution in $q / p$ becomes ever larger for very high transverse momentum particles, whose trajectories in the transverse plane asymptotically tend to a straight line as $p_{T} \rightarrow \infty$. The probability for the charge of a particle to be misidentified in the reconstruction of a track increases as a function of $p_{T}$ (less than $1 \%$ at $p_{T}=500 \mathrm{GeV}$ but rising to $14 \%$ for $p_{T}=2 \mathrm{TeV}$ for muon tracks) for the same reason [95].

The efficiency of the ATLAS ID and reconstruction algorithms to reconstruct charged particle tracks depends primarily on three properties: the particle species, the transverse momentum and the psuedo-rapidity of the particle. The track reconstruction 


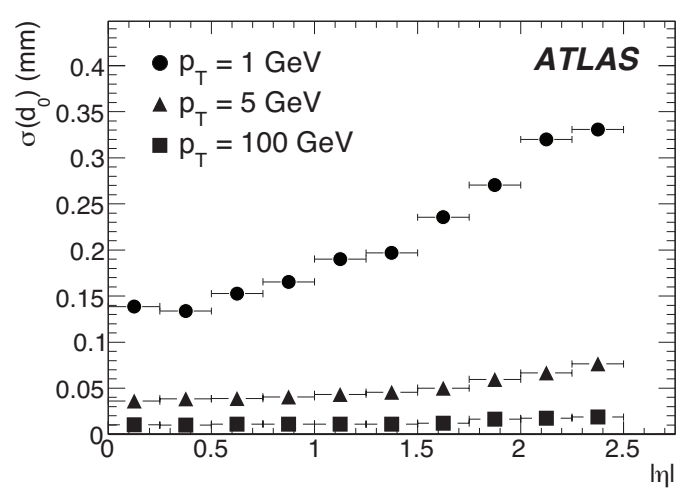

(a)

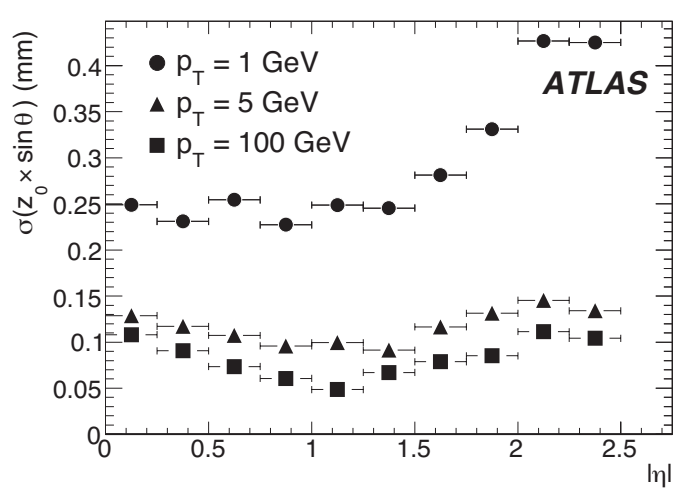

(b)

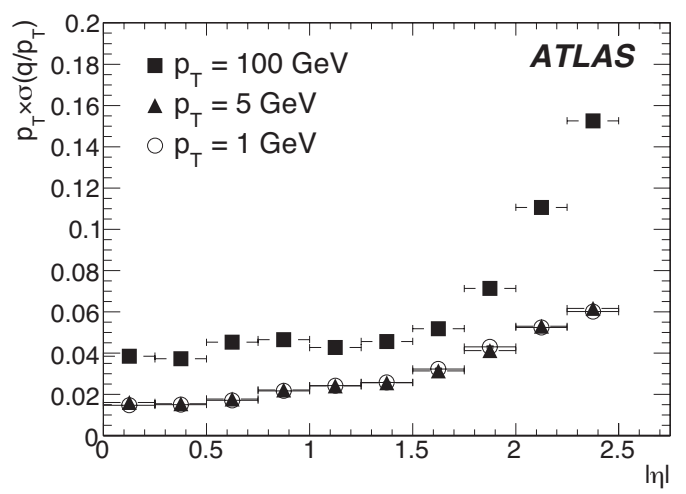

(c)

Figure 5.2: The resolutions of the track parameters (a) $d_{0}$, (b) $z_{0} \cdot \sin (\theta)$ and (c) $q / p_{T}$ (bottom) as functions of $|\eta|$, determined from MC simulation, for muon tracks with various transverse momenta. Figures taken from Ref. [95].

efficiency as a function of these three variables is shown in Figure 5.3. The different particle species interact differently with the material within the ATLAS ID due to their differing masses and properties (i.e. electromagnetic for all charged species and strong for charged pions, kaons and (anti-)protons). High energy electrons are particularly susceptible to energy losses due to bremsstrahlung $\left(e^{ \pm} \rightarrow e^{ \pm} \gamma\right)$, which can cause the electron trajectory to deviate unpredictably from the helical path expected by the reconstruction algorithm, resulting in a loss of efficiency. The tracking efficiency for electrons can be improved with the use of specifically designed track fitting algorithms (such as the Gaussian Sum Filter or Dynamic Noise Adjustment algorithms) that can account for bremsstrahlung events [95]. Charged hadrons can 
also experience energy losses due to nuclear interactions in the detector material that can perturb their trajectories or even initiate hadronic showers. There is also a slight charge asymmetry (less than 10\%) to the track reconstruction efficiency due to the different interaction cross sections with the detector material for matter and anti-matter particles. The differing coverage of the various ID sub-detectors in $|\eta|$, including the transition from the barrel to the endcaps, leads to some strong dependence of the track reconstruction efficiency on $|\eta|$. Finally, below a certain transverse momentum threshold (around $500 \mathrm{MeV}$ ) charged particles will begin to loop in the magnetic field and so not reach the outer layers of the ID, leading to fewer measurement points on the track. This results in a "turn-on" behaviour in the track reconstruction efficiency, as shown in Figure 5.3. In general, the track reconstruction efficiency is in excess of $80 \%$ for all particle species with transverse momenta in excess of $1 \mathrm{GeV}$ and higher still in specific cases (e.g. muons with $p_{T}>10 \mathrm{GeV}$ ). 


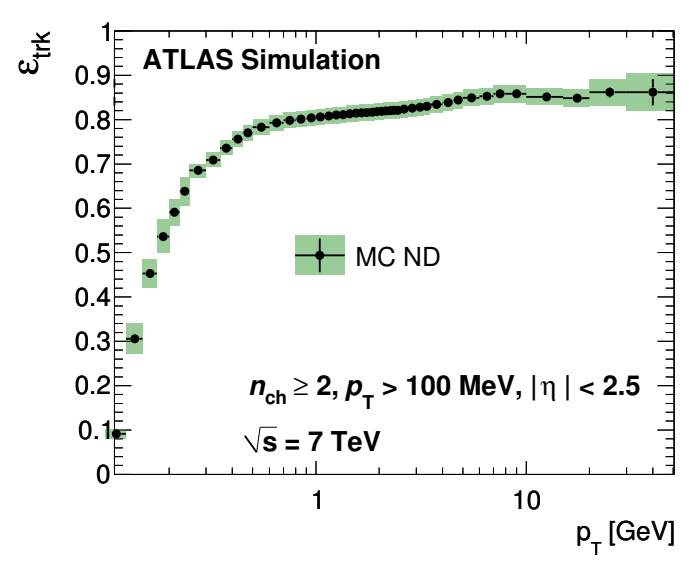

(a)

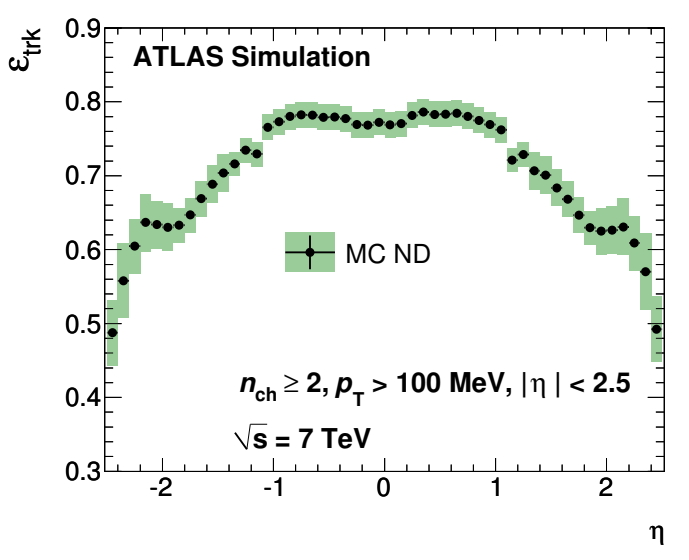

(b)

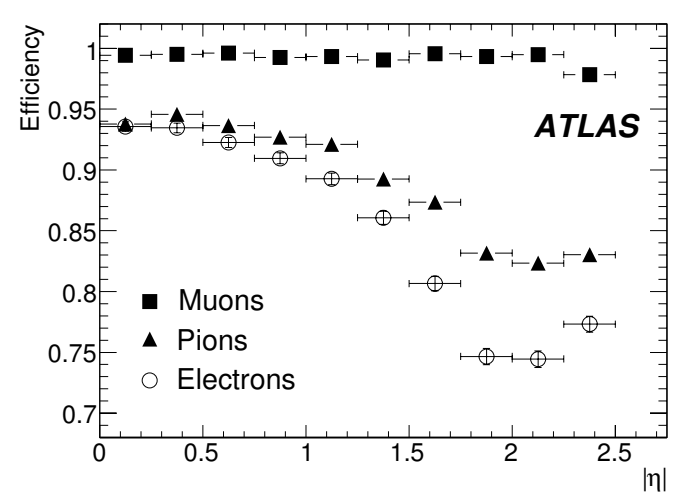

(c)

Figure 5.3: The track reconstruction efficiency for charged particles as a function of $p_{T}$ (a) and $\eta$ (b) for charged particles produced in non-diffractive (ND) minimum bias events in MC simulation [105]. The track reconstruction efficiency as a function of $|\eta|$ for three charged particle species (c) with transverse momentum $p_{T}=5 \mathrm{GeV}$, derived from simulation [95]. 


\subsection{Muon Reconstruction}

The muon system of the ATLAS detector provides coverage for muon reconstruction with high efficiency within the pseudo-rapidity region $|\eta|<2.3$ and over a large transverse momentum range from $3 \mathrm{GeV}$ to nearly $1 \mathrm{TeV}$. The technical details of the ATLAS muon system are described in Section 4.5. It constitutes a system of precision tracking chambers immersed in a magnetic field provided by a set of superconducting magnets that surround the calorimeter cryostat. These superconducting toroid magnets are arranged in a large barrel section covering the region $|\eta|<1.4$ and two endcap sections that together cover $1.6<|\eta|<2.7$. The barrel section provides an integrated magnetic field strength of between 1.5 to $5.5 \mathrm{Tm}$ while the endcaps provide between 1.0 to $7.5 \mathrm{Tm}$, both in the $r-z$ plane [93]. The precision tracking detectors (mostly MDT chambers, supplemented with CSC chambers in the forward region) are arranged in three stations, as shown in Figure 5.4, and provide measurements of the muon direction in $\eta$. The trigger detectors (TGCs and RPCs) also provide supplementary rough muon position measurements that can be used in offline muon reconstruction algorithms. The ATLAS Inner Detector also plays an important role in muon reconstruction and offers more precise momentum measurements for very low transverse momentum muons $\left(p_{T}<20 \mathrm{GeV}\right)$. In certain cases, measurements in the calorimeters are also used as input to muon identification algorithms.

The muon momentum resolution varies from around $4 \%$ to over $10 \%$ for very high transverse momentum muons. The limiting factors affecting the momentum resolution achievable by the muon system vary dramatically as a function of muon transverse momentum. Energy loss in the detector material traversed before reaching the muon system dominates the momentum resolution for muons with transverse momentum below $300 \mathrm{GeV}$. At higher $p_{T}$, the inherent characteristics of the muon detectors become the dominant factor and the relative momentum resolution de- 


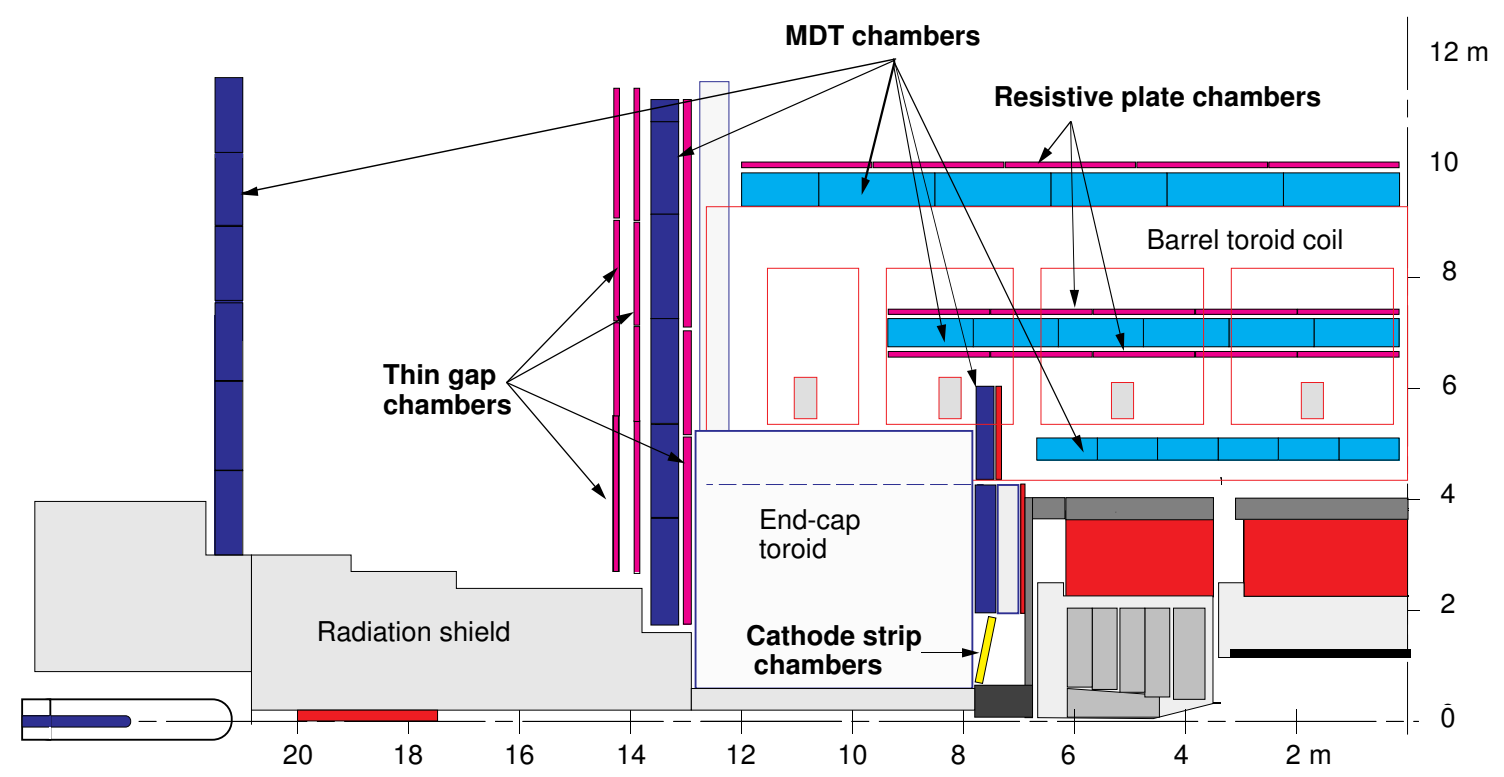

Figure 5.4: A schematic diagram of the ATLAS muon system showing the three stations of tracking and trigger detectors. Image from Ref. [95].

creases asymptotically for muons with transverse momentum approaching $1 \mathrm{TeV}$.

Several muon identification and reconstruction algorithms are employed in ATLAS, of which each exhibits specific benefits and limitations. Three different approaches are used to identify muons, leading to three types of identified muons: Standalone, Combined and Tagged (see below). Two independent classes of muon reconstruction algorithm, Staco and Muid, are then used to form reconstructed muons that can be used in physics analyses. Each muon identified by one of the three approaches is processed by both of these reconstruction algorithms, leading to two reconstructed objects being stored in the collected datasets for each physical muon. The Staco reconstruction algorithm uses a statistical combination of the tracks measured in the MS and ID, based on their independently determined track covariance matrices. The Muid reconstruction algorithm refits the muon track from the individual track hits in both the MS and the ID. The three muon types are summarised below.

Standalone: Muons identified from tracks reconstructed in the MS alone are known as standalone muons. This approach first identifies track segments in each of 
the three muon stations, which are subsequently linked to form standalone muon tracks. This track is then used to extrapolate the muon trajectory back to the interaction point, accounting for the energy losses expected in the traversed material (dominated by the calorimeter). The standalone approach offers a large overall acceptance (over the full $|\eta|<2.7$ range of the MS) that is not limited by the coverage of the ID $(|\eta|<2.5)$, but it includes some regions of zero acceptance due to limited chamber coverage in the regions near $\eta \approx 0$ and $|\eta| \approx 1.2[95]$. One important limitation of the standalone approach is its reliance on the reconstruction of track segments in multiple MS stations. Lower $p_{T}$ muons, which may not penetrate far enough to be measured in all three stations, will typically suffer from a much lower identification efficiency. Further to this, the standalone approach is more susceptible to identifying non-prompt secondary muons from light meson decays produced in hadronic showers initiated in the calorimeters, due to the lack of ID information that could provide a veto.

Combined: Independent tracks reconstructed in the MS and ID, both consistent with being formed by the same charged particle, are referred to as combined muons. The compatibility of independent MS and ID tracks with a common muon hypothesis is quantified with a match $\chi^{2}$ calculated from the track parameters of both measured tracks and their respective covariance matrices [95]. Both the Staco and Muid reconstruction algorithms combine the two measurements, while taking upstream energy losses into account for the MS measurement. The combined approach typically exhibits the highest purity, as the independent ID track is effective at reducing backgrounds from hadron decays in flight and secondary muons produced in hadronic showers, but it has an acceptance limited by the coverage of the ID $(|\eta|<2.5)$.

Tagged: The tagged approach exploits information from both the ID and the MS 
in an alternative manner to the combined approach. The algorithm is seeded from ID tracks with transverse momenta large enough that they could reach the MS (typically above $3 \mathrm{GeV}$ ). These ID tracks are propagated forward to the first station of the MS, where nearby track segments are searched for. The compatibility of the MS track segment and the ID track is quantified with either a $\chi^{2}$ discriminant or a quantity derived from a neural network [95]. ID tracks that are compatible with an MS track segment constitute tagged muons. The track measured in the ID alone is used to reconstruct the muon momentum. The tagged approach provides a high efficiency for very low $p_{T}$ muons, which do not penetrate far enough to leave hits in all three MS stations, or to form an independent MS track.

The total efficiency for the identification and reconstruction of muons varies significantly between the different algorithms and as a function of muon $\eta$ and $p_{T}$. Muons identified with the combined approach and reconstructed with the Staco algorithm are typically used for most ATLAS quarkonium studies, including the measurements presented in Chapters 6 and 7. This combination of algorithms has a total efficiency that approaches a plateau in excess of $95 \%$ in the regions with good muon chamber coverage (outside $|\eta|<0.1$ and $1.1<|\eta|<1.3$ ), as shown in Figure 5.5.

The reconstructed momentum, for muons with transverse momenta relevant to quarkonium studies in ATLAS (i.e. from $J / \psi \rightarrow \mu^{+} \mu^{-}$and $\Upsilon(n S) \rightarrow \mu^{+} \mu^{-}$), is determined from the ID track parameters alone, as these measurements are not affected by energy losses in the calorimeters and provide the most precise determination of the muon momentum. The resolution of the momentum and reconstructed track parameters is therefore entirely determined by the ID and track reconstruction algorithms which are discussed in Section 5.1, with the MS only serving as a muon "tagger". 


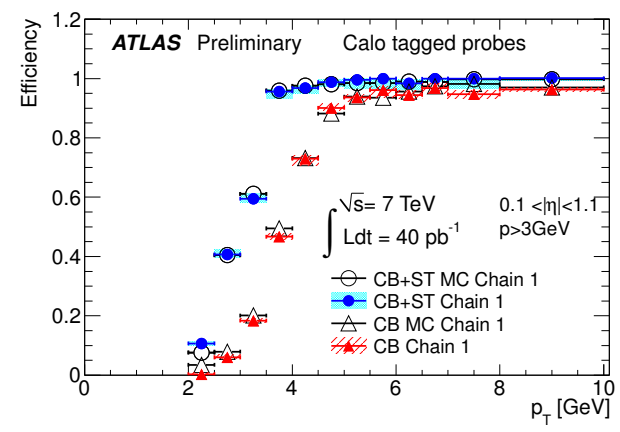

(a)

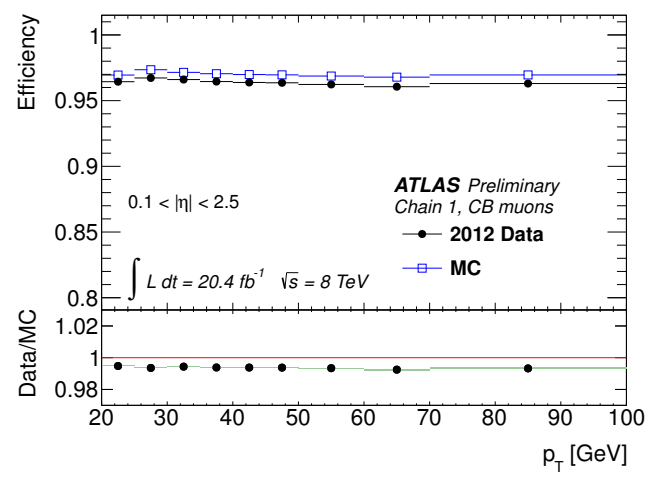

(c)

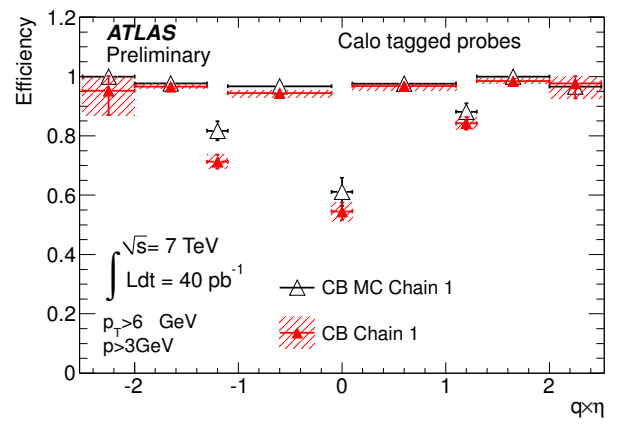

(b)

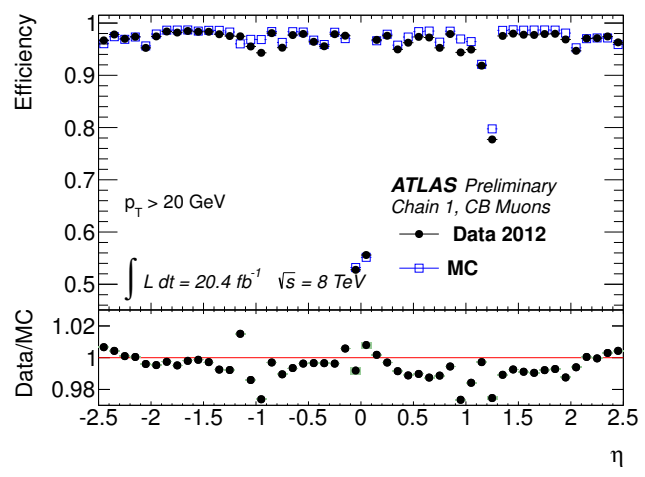

(d)

Figure 5.5: The reconstruction efficiency for muons reconstructed with the combined (CB) approach and Staco algorithm (denoted chain 1). The efficiency is measured in data with $J / \psi \rightarrow \mu^{+} \mu^{-}$events (a and b) and $Z \rightarrow \mu^{+} \mu^{-}$events (c and d) and is compared to that derived in MC simulation. Figures taken from Refs. [106] and [107]. 


\subsection{Photon Conversions}

The interaction of electrons or photons with the inactive material of a particle physics detector is a common phenomenon that has important consequences for the design and performance of an experiment. While these interactions ultimately lead to energy losses or the destruction of particles of interest, which can be an undesirable effect, they are exploited in certain circumstances to serve as a useful experimental tool.

The interactions of high energy photons and electrons with matter proceed through the photon conversion (or pair production, $\gamma \rightarrow e^{+} e^{-}$) and bremsstrahlung $\left(e^{ \pm} \rightarrow e^{ \pm} \gamma\right)$ processes, respectively. The energy loss experienced by a high energy photon or electron as it travels through matter is characterised by the radiation length $X_{0}$. This is defined as the mean distance over which a high energy electron loses $1 / e$ of its initial energy due to the bremsstrahlung process [3]. The same quantity also describes approximately $7 / 9$ of the mean free path for a high energy photon to interact with matter via the photon conversion process [3]. The radiation length, measured in units of $\mathrm{g} \mathrm{cm}^{-2}$, is uniquely defined for a given material and has a strong dependence on the atomic number $Z$ of the material. For example, the length $X_{0}$ for Silicon $(Z=14)$ is approximately $9.4 \mathrm{~cm}$.

The photon conversion process involves the interaction of a photon of energy $E>2 m_{e}$ (where $m_{e}=0.511 \mathrm{MeV}$ is the mass of the electron) with an atomic nucleus (or electron) leading to the production of an electron-positron pair $\left(\gamma A \rightarrow e^{+} e^{-}\right)$. This does not occur as an (real) observable process spontaneously in the vacuum as the presence of the nucleus (or other strong external electric field) is required to allow energy and momentum to be conserved in the interaction. The dominance of this process at high energies is manifest in the total cross section for photon-nucleus scattering, as shown in Figure 5.6. 


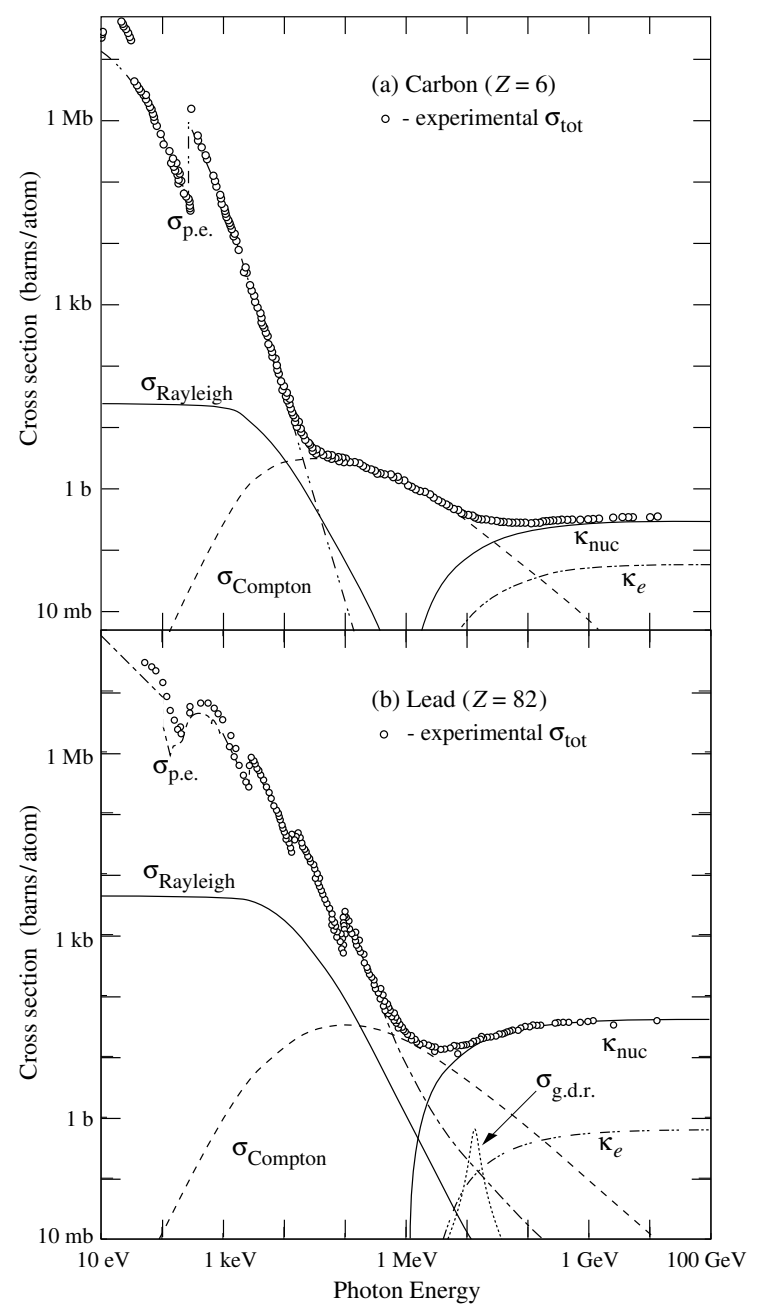

Figure 5.6: The total cross sections for the interaction of photons with material (Carbon and Lead) as functions of energy. The cross section is dominated by the photo-electric effect $\left(\sigma_{\text {p.e. }}\right)$ at low energy with small contributions from Rayleigh and Compton scattering at energies below $1 \mathrm{MeV}$. The contributions $\kappa_{\text {nuc }}$ and $\kappa_{e}$ denote the cross sections for the pair production of $e^{+} e^{-}$pairs from the interaction of a photon with an atomic nucleus and an electron, respectively, and represent the dominant contributions for $E>1.02 \mathrm{MeV}$. Figure from Ref. [3].

In the high energy limit, the differential cross section for photon conversion is roughly independent of photon energy and is given by,

$$
\frac{d \sigma}{d x}=\frac{A}{X_{0} N_{A}}\left[1-\frac{4}{3} x(1-x)\right]
$$

where $x=E_{e^{-}} / E_{\gamma}$ is the fraction of the photon's energy carried by the electron, $A$ is the atomic mass of the material, $X_{0}$ is the radiation length of the material and 
$N_{A}$ is Avagadro's number [108]. Equation 5.1 can then be integrated over $x$ to give an approximate value for the total cross section of $\sigma=(7 / 9)\left(A / X_{0} N_{A}\right)$, valid for energies in the plateau region of Figure 5.6 above $1 \mathrm{GeV}[3]$. The differential cross section is shown in Figure 5.7, demonstrating the preference for the interaction to produce an $e^{+} e^{-}$pair with an asymmetric sharing of the initial photon energy.

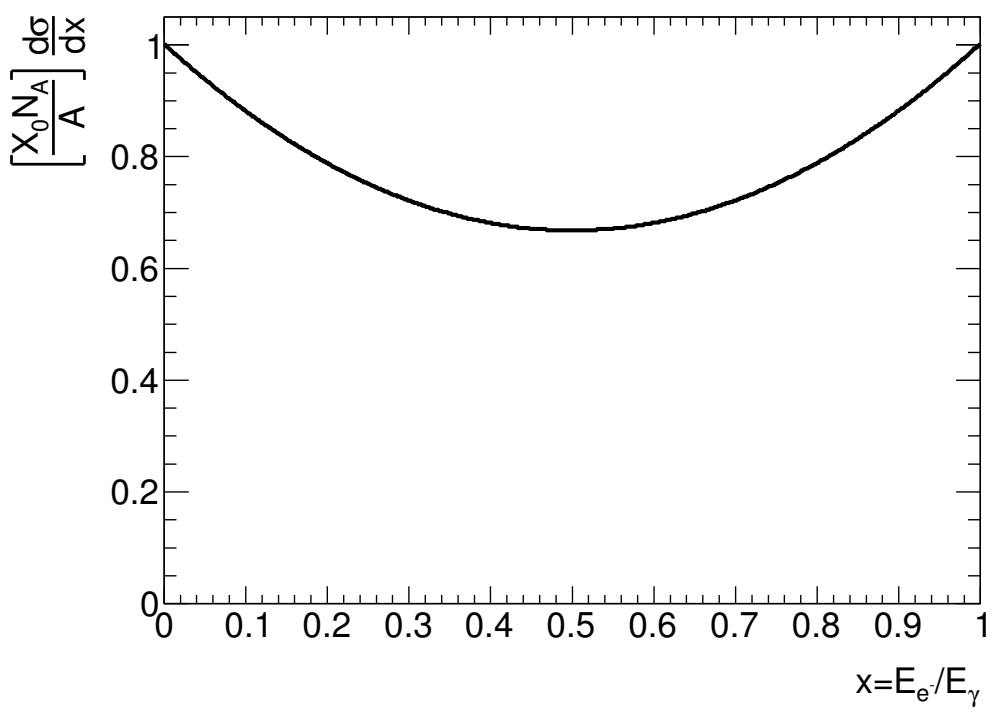

Figure 5.7: The differential cross section for photon conversion, normalised by a material dependent pre-factor, as a function of the energy sharing parameter $x$. Information from [3].

The ATLAS detector contains a significant amount of both active (instrumented) and inactive material. The material budget of the ATLAS inner detector, measured in radiation lengths, is shown as a function of $|\eta|$ in Figure 5.8. Across the full $|\eta|$ range of the ID, there is at least half a radiation length between the beam line and the calorimeters. The probability for an electron or photon to interact before reaching the calorimeters is therefore large and must be accounted for in the calorimeterbased electron and photon reconstruction algorithms. This interaction is perhaps more consequential for photons, since the initial photon is lost in a conversion event, while an electron bremsstrahlung event does not result in the complete loss of the electron. The probability for a photon to convert, as a function of the inner detector material traversed is shown in Figure 5.9. Figures 5.8 and 5.9 demonstrate that 
there is a very significant probability for photons to convert even in the inner layers of the ID, between around $8 \%$ to $25 \%$ for photons traversing only the three pixel layers. The $e^{+} e^{-}$pairs produced by photons that convert within these layers (or in the SCT layers) can be reconstructed from tracks in the SCT and TRT layers of the ID, allowing the momentum of the initial photon to be determined. This method often provides a measurement of photon momentum much more precise than a direct calorimetric measurement, particularly for photons with low transverse momentum. The reconstruction of photon conversions is a useful tool that can be exploited to perform measurements of processes involving photons.

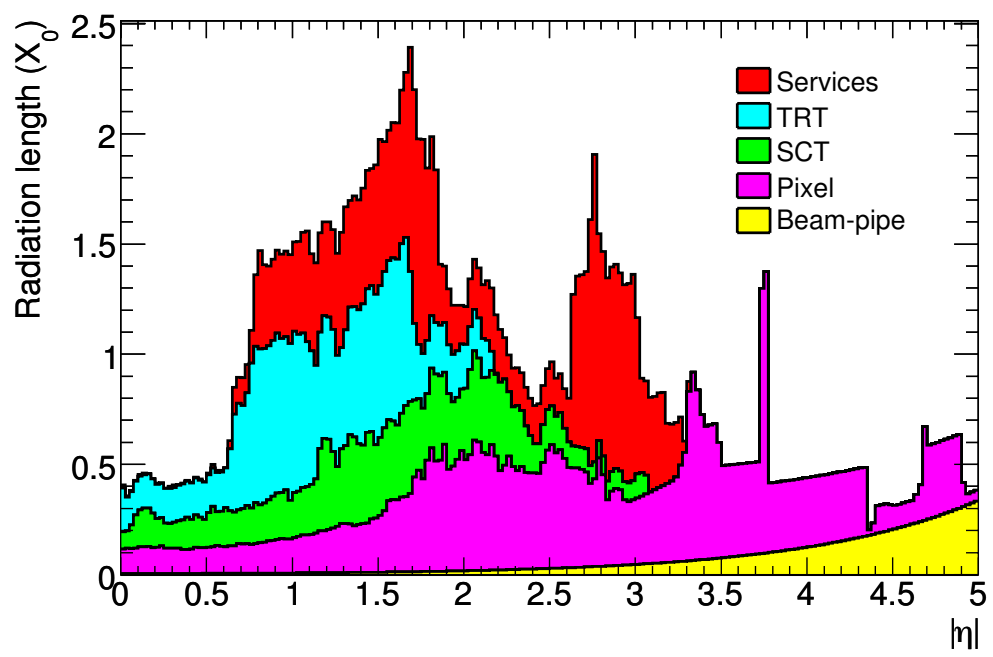

Figure 5.8: The material budget of the ATLAS inner detector (up to the solenoid) measured in radiation lengths as a function of $|\eta|$, broken down into the individual detector components. Figure taken from Ref. [95]. 


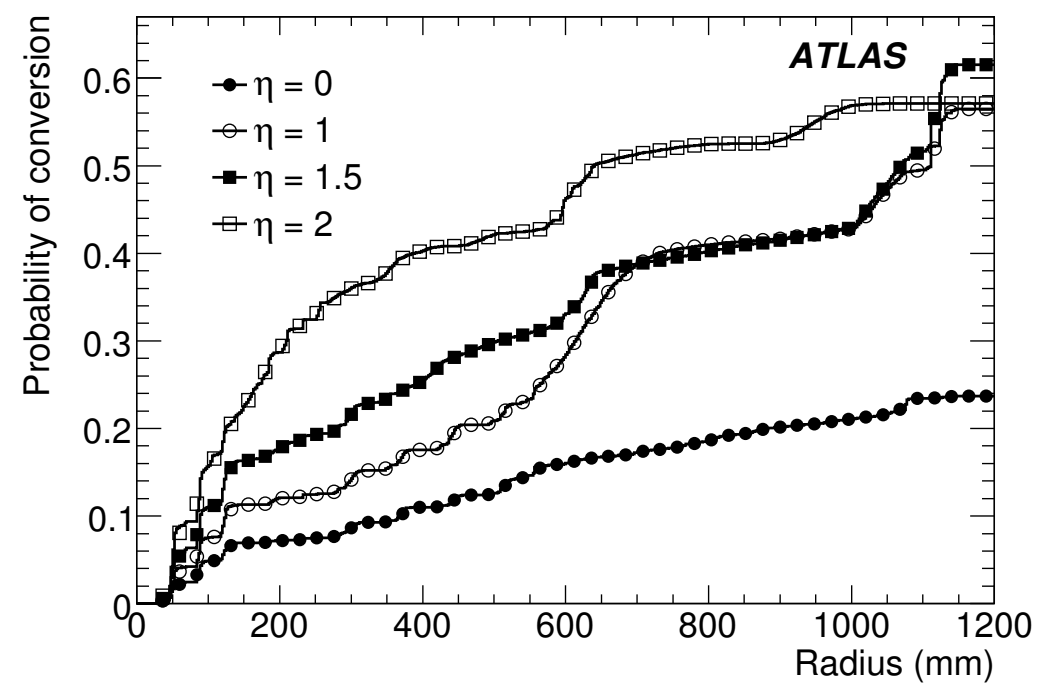

Figure 5.9: The photon conversion probability (for photons with transverse momentum in excess of $1 \mathrm{GeV}$ ) as a function of inner detector material traversed, for several trajectories in $\eta$, derived from simulation. Figure taken from Ref. [95]. 


\subsubsection{Photon Conversion Reconstruction in ATLAS}

Photon conversions can be reconstructed from $e^{+} e^{-}$track pairs in the ATLAS ID alone, with no input from the calorimeters. This method is very efficient and precise for low $p_{T}$ photons (below around $20 \mathrm{GeV}$ ). The matching of the $e^{+}$and $e^{-}$tracks from conversions to energy deposits in the EM calorimeter can also be performed for higher $p_{T}$ photons to improve the resolution and purity. The reconstruction of photon conversions in the ATLAS ID proceeds through three main stages: the selection of charged particle tracks, the selection of track pairs and the fitting of the conversion vertex.

Track Selection: Individual reconstructed tracks that are consistent with having originated from photon conversions are first selected from the full collection of tracks reconstructed in the ID. Upper limits on their transverse and longitudinal impact parameters $d_{0}$ and $z_{0}$ are imposed and tracks with TRT hits are required to satisfy a loose requirement on the electron ID probability (based on the fraction of high threshold TRT hits) [95].

Track Pair Selection: Each pair of oppositely charged tracks that satisfy the track selection cuts are considered for further analysis. The initial track pairs are classified into three groups based on their sub-detector hit information: Si-Si pairs (both tracks have hits in the silicon ( $\mathrm{Si}$ ) detectors), Si-TRT (one track was reconstructed in the TRT alone) and TRT-TRT (both tracks were reconstructed in the TRT alone). A series of requirements, which vary across the three classes of track pair, are then imposed to reduce the contamination from background sources [95]:

- Polar angle difference: The difference between the polar angles of the two reconstructed tracks is required to be small. This quantity is consistent with zero for optimally reconstructed tracks from genuine conversions. 
- Distance of minimum approach: Track pairs from genuine photon conversions have an opening angle in the transverse plane that is consistent with zero. The distance between the helices of the two tracks is thus required to be small (within detector resolution) at the point of closest approach.

- First hit distance: The first detector hits on each of the two tracks are required to be spatially close. This requirement is most effective for TRTTRT track pairs.

- Arc length: Requirements are imposed on the arc lengths of the track helices, projected onto the transverse plane, and measured between the line connecting the centres of the two track circles and their intersection points [95]. The arc length should be small for genuine photon conversions.

The specific requirements on these quantities are based on studies using MC simulation and are chosen to be loose enough that the effects of electron bremsstrahlung do not result in significant losses in reconstruction efficiency.

Vertex Fitting: Finally, the vertex of the track pair is fitted, taking into account the detector material traversed and the track perigee parameters calculated at this estimated intersection point. Track pairs that are successfully fitted are subjected to a number of final requirements based upon the parameters of the fitted vertex. The invariant mass of the candidate $e^{+} e^{-}$conversion pair is required to be small and a quality requirement on the $\chi^{2}$ of the fitted vertex is also imposed.

The candidate photon conversions reconstructed with this method typically have a high purity (particularly for Si-Si pairs) with the main background being prompt $e^{+} e^{-}$pairs from $\pi^{0} \rightarrow \gamma e^{+} e^{-}$decays, which can be rejected with a cut on the radius of the reconstructed $e^{+} e^{-}$vertex. 


\subsection{Unconverted Photon Reconstruction}

Reconstructed photon objects are seeded from clusters of energy deposits in the ATLAS EM calorimeter, where a transverse energy $E_{T}^{\gamma}>2.5 \mathrm{GeV}$ is measured within a collection of $\eta \times \phi=3 \times 5$ calorimeter cells in the second layer of the EM calorimeter. A cluster is considered as a candidate unconverted photon if none of the tracks reconstructed in the inner detector spatially matches the cluster when extrapolated to the EM calorimeter. The reconstruction of photons in the ATLAS detector and the various photon identification criteria used in physics analyses are described in detail in [109].

The studies presented in Chapter 6 use a set of photon identification criteria denoted "loose". These criteria are based on the shape of the shower in the EM calorimeter and information from the hadronic calorimeter [109]. The selection makes use of three shower quantities:

- $R_{\text {had }}$ (hadronic leakage): $R_{\text {had }}$ is defined as the ratio of transverse energy deposited in the first layer of the hadronic calorimeter (in the cell behind the candidate photon cluster) to the total transverse energy of the photon candidate. Genuine photons typically have a $R_{\text {had }}<2 \%$ [109].

- $R_{\eta}$ : The ratio of the energy deposited in $\eta \times \phi=3 \times 7$ calorimeter cells to the energy deposited in $\eta \times \phi=7 \times 7$ calorimeter cells in the second layer of the EM calorimeter [109].

- $w_{2}$ : The RMS of the energy distribution in consecutive cells in $\eta$ in the second layer of the EM calorimeter [109].

The "loose" photon identification criteria are designed to select narrow EM showers consistent with being genuine photons while simultaneously rejecting showers initiated by hadronic jets. The specific requirements on these quantities are obtained from MC simulation. 


\subsection{Triggering of Quarkonium Decays $\mathcal{Q} \rightarrow \mu^{+} \mu^{-}$}

The di-muon decays of the vector quarkonium states, $\mathcal{Q} \rightarrow \mu^{+} \mu^{-}$, provide the most convenient signature to trigger upon events containing quarkonium states produced in $p p$ collisions. The ATLAS detector is equipped with dedicated muon trigger detectors. The di-muon signature is experimentally clean and the presence of two kinematically correlated muons can be exploited to reduce the background rate substantially.

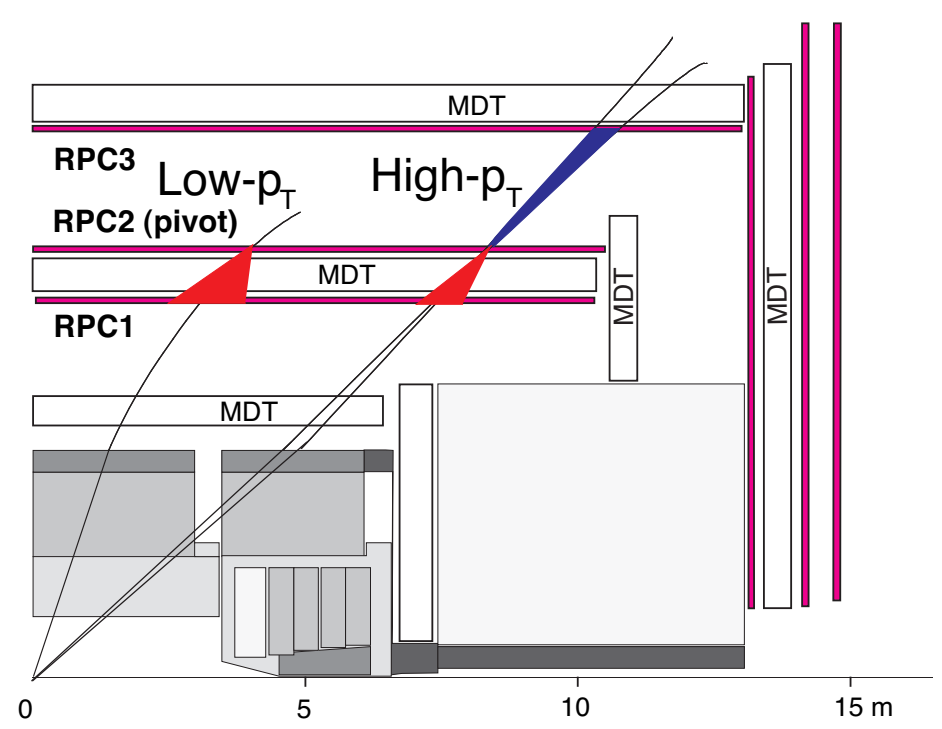

Figure 5.10: A schematic diagram of the ATLAS muon trigger detectors. The diagram shows two examples of low $p_{T}$ trigger roads (red) between the first and second stations and a high $p_{T}$ extension road (blue) between the second and third stations. Image from Ref. [93].

The ATLAS muon trigger detectors are arranged in three stations (RPCs in the barrel and TGCs in the endcaps), each of which contains two active layers, as shown in Figure 5.10 for the barrel detectors. The ATLAS muon trigger operates by searching for hit coincidences in these stations within pre-defined roads, which represent the path of a muon with a given lower $p_{T}$ threshold [93]. The widths of the trigger roads vary as a function of the $p_{T}$ threshold; lower $p_{T}$ thresholds have a broader road width to account for the greater bending the muon trajectories experience in the magnetic field. Three low $p_{T}$ thresholds and three high $p_{T}$ thresholds 
can be defined. The L1 muon trigger is seeded by hits in the second station layers. Hits are then searched for in the first station layers within a road defined by the $p_{T}$ threshold of the trigger, taking the positions of the hits in $\eta$ and $\phi$ into account. Finally, the trigger requires a hit coincidence in three of the four layers, whereupon an L1 ROI in $\eta$ and $\phi$ is passed to the HLT [93]. High $p_{T}$ muon triggers also require coincident hits (one of a possible two) in the third (outer) station of the trigger detectors, in addition to a successful low $p_{T}$ coincidence. Muons from $\mathcal{Q} \rightarrow \mu^{+} \mu^{-}$ events typically have low $p_{T}$ and the ATLAS quarkonium triggers typically require muon $p_{T}$ thresholds of 4 and $6 \mathrm{GeV}$ at L1.

The ATLAS di-muon quarkonium triggers employed during the 2011 and 2012 LHC runs exploit the di-muon signature of $\mathcal{Q} \rightarrow \mu^{+} \mu^{-}$decays directly in the L1 trigger and require at least two independent L1 muon ROIs. This helps to control the trigger rate and to reduce the backgrounds. Triggers that require at least two independent L1 ROIs are referred to as topological triggers. Other approaches also exist, such as the so called "TrigDiMuon" triggers, which require only a single L1 ROI with a second being searched for at L2. Such triggers typically have a higher efficiency but also exhibit higher L1 rates and so were only used during the LHC commissioning run of 2010, when the instantaneous luminosity was low. The full detector information, including ID tracks, is used at the HLT level, where a vertex fit is performed to the two muons. The HLT requires that the two muons have opposite charges and imposes a loose requirement on the quality of the vertex fit. Loose invariant mass cuts can also be applied at the HLT to select only $J / \psi$ and $\psi(2 S)$ or $\Upsilon(1,2,3 S)$ regions, to reduce the background rate from non-resonant dimuon production and double semi-leptonic decays of $b \bar{b}$ pairs. Figure 5.11 shows a comparison of the event samples collected by the various dedicated quarkonium triggers implemented in ATLAS with the more generic high $p_{T}$ single muon triggers. The dedicated quarkoium triggers with low $p_{T}$ thresholds significantly improve the acceptance for $J / \psi \rightarrow \mu^{+} \mu^{-}$and $\Upsilon(n S) \rightarrow \mu^{+} \mu^{-}$events. 


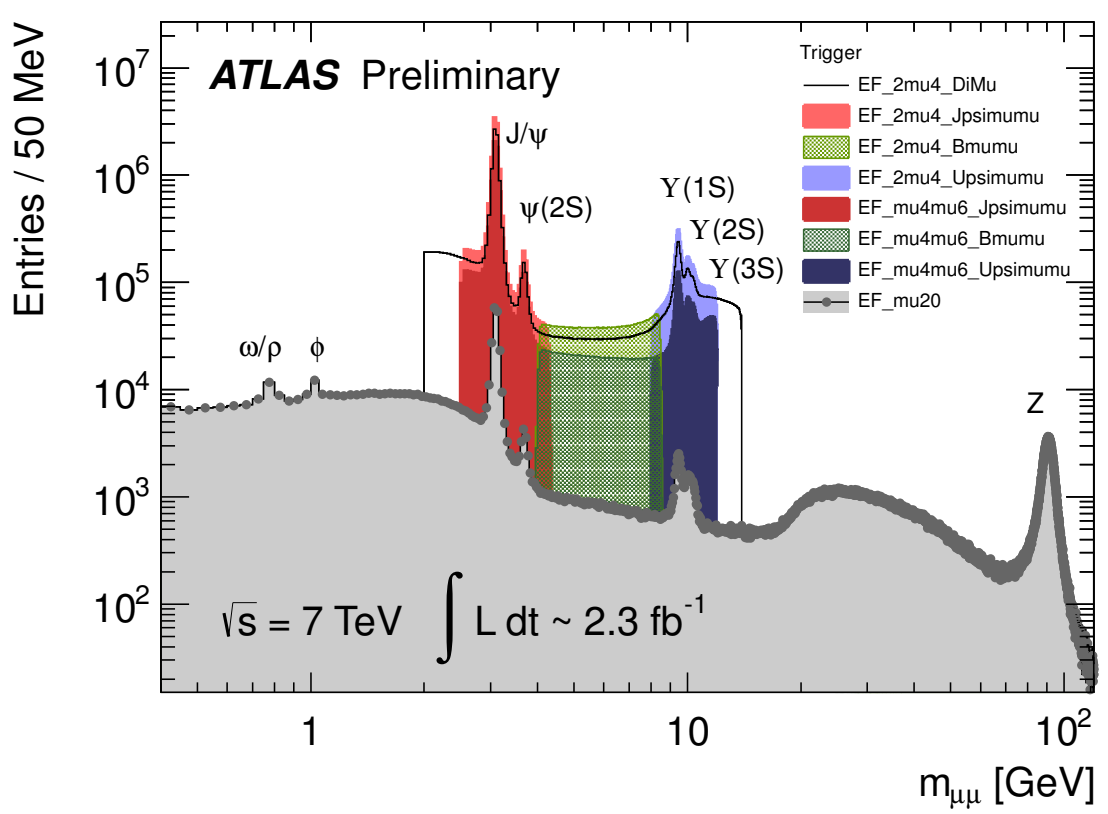

Figure 5.11: A comparison of the data samples collected by various ATLAS muon triggers. The dedicated low $p_{T}$ di-muon triggers (coloured histograms) significantly improve the acceptance for $J / \psi \rightarrow \mu^{+} \mu^{-}$and $\Upsilon(n S) \rightarrow \mu^{+} \mu^{-}$events in comparison to a single high $p_{T}$ muon trigger (grey histogram). The shaded histograms represent the number of events collected by triggers with different muon $p_{T}$ threshold and di-muon invariant mass cuts. Figure from Ref. [103]. 


\subsection{Quarkonium Decay Reconstruction Techniques}

This section introduces some common analysis methods often deployed in quarkonium production analyses at hadron collider experiments. In particular, techniques that are used in several of the measurements presented in Chapters 6 and 7 are summarised here.

\subsubsection{Mass difference distributions}

As discussed in Chapter 3 , the $\chi_{c}$ and $\chi_{b}$ states play an important role in the phenomenology of quarkonium production at hadron colliders. It is often advantageous to be able to study the angular momentum states of a $\chi_{J}$ triplet individually. The three states $(J=0,1,2)$ differ physically only in their total angular momenta $J$ and masses. The determination of the total angular momentum of a particle generally requires a complicated analysis of the angular distributions of its decay products, while the invariant mass of its reconstructed decay products is generally much simpler to study and interpret. It is thus helpful in experimental studies of the $\chi_{J}$ states to be able resolve an individual peak in invariant mass distributions for each $\chi_{J}$ state. The mass splittings between the individual angular momentum states within a $\chi_{c J}$ or $\chi_{b J}$ quarkonium triplet are very small, between around 10-50 MeV depending upon the system, making their separation challenging at modern hadron collider experiments which are typically not optimised for such studies.

One method that is often employed to achieve a reconstructed mass distribution with a resolution fine enough to separate the individual $\chi_{J}$ states is the use of a mass difference distribution. The method exploits the fact that one or more invariant mass difference(s) can be defined for a decay chain involving two (or more) consecutive decays. The simplest case is the decay of a particle $P_{1} \rightarrow P_{2}+A$, where $P_{2}$ subsequently decays by $P_{2} \rightarrow B+C$; real examples include $\chi_{c J} \rightarrow J / \psi \gamma$ with $J / \psi \rightarrow \mu^{+} \mu^{-}$, and $D^{* \pm} \rightarrow D^{0} \pi^{ \pm}$with $D^{0} \rightarrow(K \pi)^{0}$. The difference between the 
invariant masses $m(A B C)-m(B C)$ (e.g. $\left.m\left(\mu^{+} \mu^{-} \gamma\right)-m\left(\mu^{+} \mu^{-}\right)\right)$is itself Lorentz invariant and is equal to $M\left(P_{1}\right)-M\left(P_{2}\right)$. Consequently, reconstructed invariant mass difference distributions exhibit peaks corresponding to definite mass states in the same way that invariant mass distributions do. In the case that the decay products $A B C$ are reconstructed with some measurement error, perhaps due to energy losses in detector material or due to the inherent resolution of the detector, the propagated effect on $m(A B C)$ is directly correlated with the effect on $m(B C)$. For example, if $m(B C)$ is reconstructed at a mass slightly higher than $M\left(P_{2}\right)$ then $m(A B C)$ will also be reconstructed at a mass higher than $M\left(P_{1}\right)$ (assuming $C$ is perfectly reconstructed). However, this upward mass shift is partially cancelled in the mass difference $m(A B C)-m(B C)$. The overall mass difference distribution will exhibit a peak around $M\left(P_{1}\right)-M\left(P_{2}\right)$, with an effective mass resolution that is smaller than that observed for the three body invariant mass, $m(A B C)$. This method can also be extended to multi-body and cascade decays with the construction of an appropriate mass difference parameter.

The improvement in mass resolution achieved by the use of the mass difference can be demonstrated by a simple simulation, as shown in Figure 5.12. This simulation generates $\chi_{c 1} \rightarrow J / \psi \gamma \rightarrow \mu^{+} \mu^{-} \gamma$ decays and adjusts the transverse momentum of both muons and the photon by a randomly chosen multiplicative factor that is gaussian distributed with a mean of 1 and a width chosen roughly to mimic the performance of the ATLAS detector ( $2 \%$ for muons and for photon conversions reconstructed from ID tracks, and $5 \%$ for low $p_{T}$ photons reconstructed in the calorimeter). The $\eta$ and $\phi$ components of the four momenta are not changed and the natural width of the $\chi_{c 1}(\Gamma=0.86 \pm 0.05 \mathrm{GeV}[3])$ is not simulated. The resulting three-body invariant mass $m\left(\mu^{+} \mu^{-} \gamma\right)$ distribution and the mass difference, $m\left(\mu^{+} \mu^{-} \gamma\right)-m\left(\mu^{+} \mu^{-}\right)+m_{J / \psi}$, distributions are shown in Figure 5.12 for photons with $p_{T}$ resolutions of both $2 \%$ and $5 \%$. In both cases, the mass resolution in the mass difference distribution is significantly narrower. This method is most effective 
in the case that the muon momentum resolution represents the dominant contribution to the overall 3-body invariant mass resolution. 

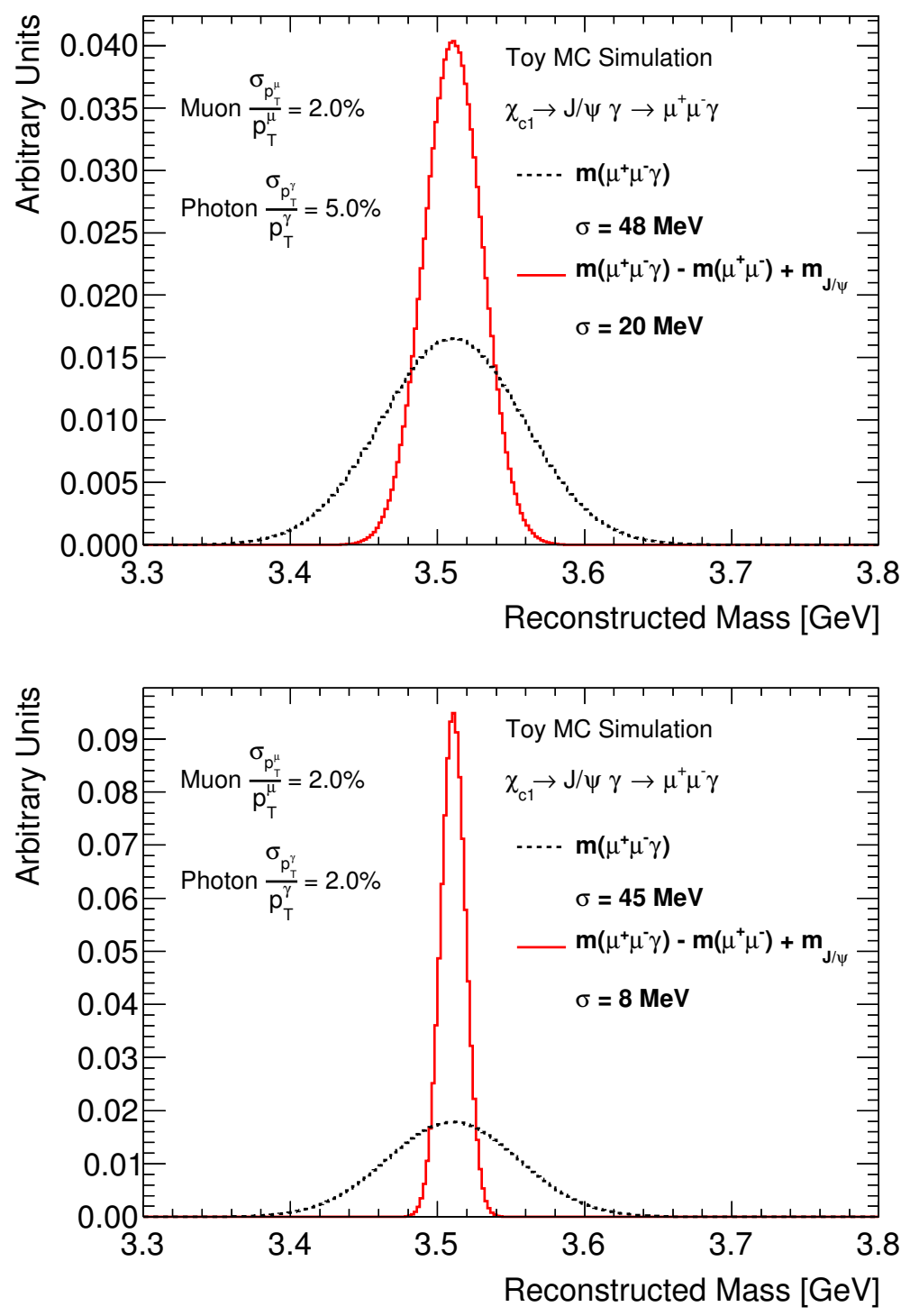

Figure 5.12: A demonstration of the resolution improvement gained with mass difference technique in simulated $\chi_{c} \rightarrow J / \psi \gamma \rightarrow \mu^{+} \mu^{-} \gamma$ events. The simulation result in the upper figure is processed with a photon transverse momentum resolution of $5 \%$ (a figure comparable to that achievable with the ATLAS calorimeter) while the lower figure is processed with a photon transverse momentum resolution of $2 \%$ (a figure comparable to that achievable through the reconstruction of photon conversions in the ATLAS ID). 


\subsubsection{Measurement of non-prompt charmonium production}

As discussed in Chapter 3, it is often very desirable to be able to quantify what fraction of charmonium states produced in hadronic collisions are produced in the decays of $b$-hadrons. This can be achieved by exploiting the long lifetime of the $b$ hadrons, typically between 1 to 2 ps. Over one third of $b$-hadrons (with $p_{T} \approx 5 \mathrm{GeV}$ ) will travel distances in the transverse plane in excess of $0.5 \mathrm{~mm}$ from their production vertex before they decay, a distance that can be well resolved by modern precision tracking detectors. Decay length or decay time variables can be defined to quantify this flight distance from the tracking measurements. These quantities are generally expressed in terms of the momentum of the reconstructed charmonium state and the position of its decay vertex relative to the primary $p p$ collision vertex. Such variables do not represent a direct measurement of the proper decay time or distance of the $b$-hadron (since all of the $b$-hadron decay products, and thus the $b$-hadron momentum, are often not reconstructed) but are directly correlated and equally as useful for the identification of non-prompt production. One such variable, defined for $J / \psi \rightarrow \mu^{+} \mu^{-}$and $\psi(2 S) \rightarrow \mu^{+} \mu^{-}$decays, is the pseudo-proper decay time

$$
\tau=\frac{L_{x y} \cdot m\left(\mu^{+} \mu^{-}\right)}{p_{T}}
$$

where $L_{x y}$ is the projection of the vector between the $J / \psi$ or $\psi(2 S)$ decay vertex and the primary $p p$ collision vertex onto its transverse momentum $p_{T}$ defined as

$$
L_{x y}=\frac{\vec{L} \cdot \overrightarrow{p_{T}}}{\left|\overrightarrow{p_{T}}\right|}
$$

where $\vec{L}$ is a vector pointing from the primary $p p$ interaction vertex to the $\mu^{+} \mu^{-}$ vertex and $\overrightarrow{p_{T}}$ is the transverse momentum vector of the $J / \psi$ or $\psi(2 S)$ [77]. The per-candidate di-muon invariant mass $m\left(\mu^{+} \mu^{-}\right)$is often substituted with the world average mass of the $J / \psi$ or $\psi(2 S)$.

The $\tau$ distribution for promptly produced charmonium peaks around zero, while 
the distribution for charmonium produced in the decays of $b$-hadrons follows a characteristic exponential decay distribution. This distribution can be fitted to determine the fraction of charmonium produced through prompt and non-prompt mechanisms. In practice, the $\tau$ distribution is often fitted in conjunction with an invariant mass (or mass difference) distribution, to determine simultaneously the prompt and non-prompt charmonium signals and the background contributions to the reconstructed charmonium decay candidates. The shapes of the prompt and non-prompt contributions to the $\tau$ distribution are shown in the demonstration fit to simulated data shown in Figure 5.13. Provided the experimental resolution in $\tau$ is good (as shown in the example), the prompt and non-prompt contributions to the distribution can be reliably fitted. This method has been widely used in charmonium production measurements at the Tevatron [47] and at the LHC [77-79].
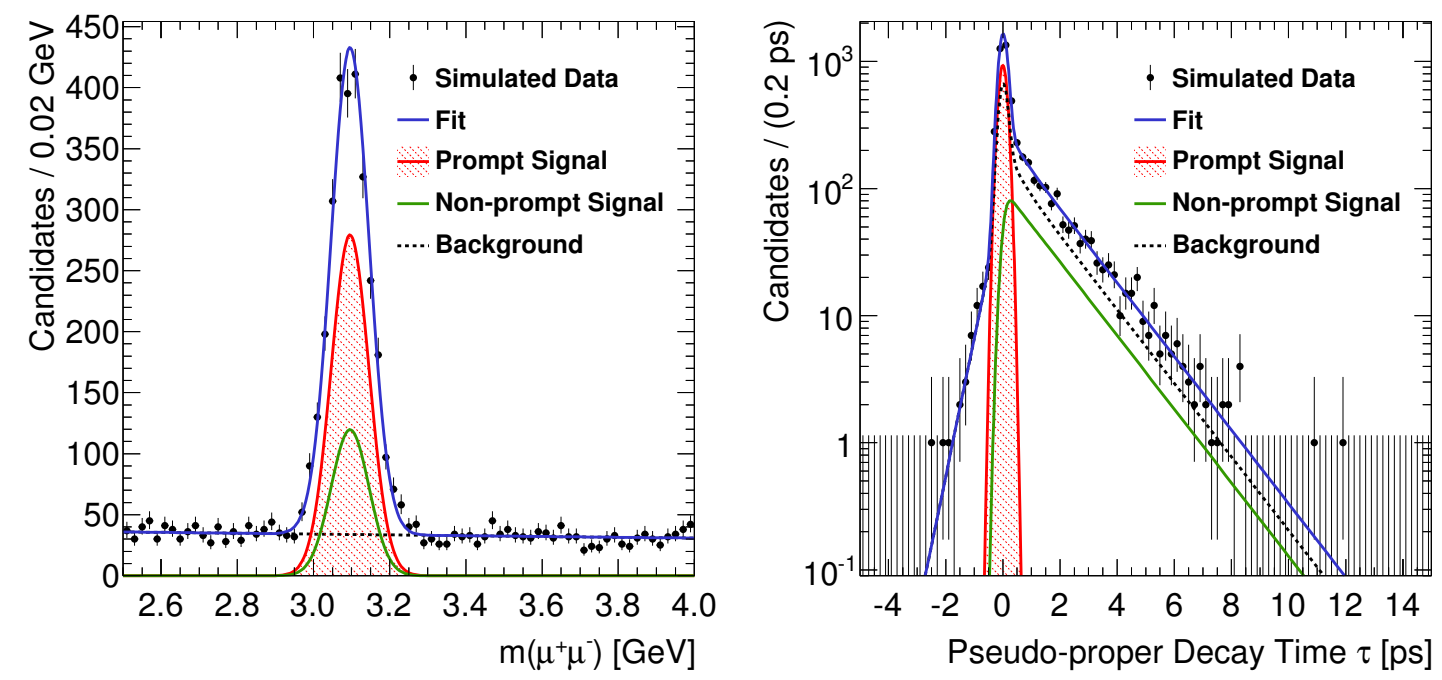

Figure 5.13: A demonstration of a simultaneous fit to the invariant mass and pseudoproper decay time distributions of reconstructed charmonium states $\left(J / \psi \rightarrow \mu^{+} \mu^{-}\right.$ in this example). The prompt (red and shaded) and non-prompt (green) yields of the charmonium state can be simultaneously determined by exploiting the discriminating power of the pseudo-proper decay time distribution. 


\subsubsection{Topological particle-vertex matching}

One ubiquitous problem in the reconstruction of particle decays is the issue of combinatoric backgrounds. In the case of $\chi_{c} \rightarrow J / \psi \gamma$, for $\chi_{c}$ produced at the LHC, the muon multiplicity for $p_{T}^{\mu}>4 \mathrm{GeV}$ in a typical $\chi_{c}$ event is reasonably low (typically only a single di-muon pair with an invariant mass broadly consistent with a $J / \psi$ ). However, the low $p_{T}$ photon multiplicity is often very high (tens of photons for each individual $p p$ interaction), due to the copious production of $\pi^{0}$ mesons which subsequently decay to two photons. Some discriminating attributes are necessary to identify the photon coming from the $\chi_{c}$ decay among the many "background" (combinatoric) photons. One quantity that is useful for this purpose, for photons reconstructed from conversions, is the 3 -dimensional impact parameter $a_{0}$ given by

$$
a_{0}=\left|\vec{x}_{e^{+} e^{-}}-\vec{x}_{\mu^{+} \mu^{-}}\right| \cdot \sin (\theta),
$$

where $\vec{x}_{e^{+} e^{-}}$and $\vec{x}_{\mu^{+} \mu^{-}}$are the positions of the conversion vertex and the $J / \psi \rightarrow$ $\mu^{+} \mu^{-}$vertex, respectively and $\theta$ is the angle between the vector $\vec{x}_{e^{+} e^{-}}-\vec{x}_{\mu^{+} \mu^{-}}$ and the converted photon momentum, as shown in Figure 5.14. This quantity is consistent with zero for photon conversions from a $\chi_{c} \rightarrow J / \psi \gamma$ decay, where the photon trajectory points back to the $J / \psi \rightarrow \mu^{+} \mu^{-}$vertex (since the $J / \psi$ decay position coincides with the $\chi_{c}$ decay position within the resolution of the detector). Requiring that $a_{0}$ be small can reject converted photons that are not consistent with having originated from a given vertex (e.g. photons coming from pileup interactions). The implementation of this method in the selection of $\chi_{c} \rightarrow J / \psi \gamma$ and $\chi_{b} \rightarrow \Upsilon \gamma$ decays is discussed in Chapters 6 and 7 . 


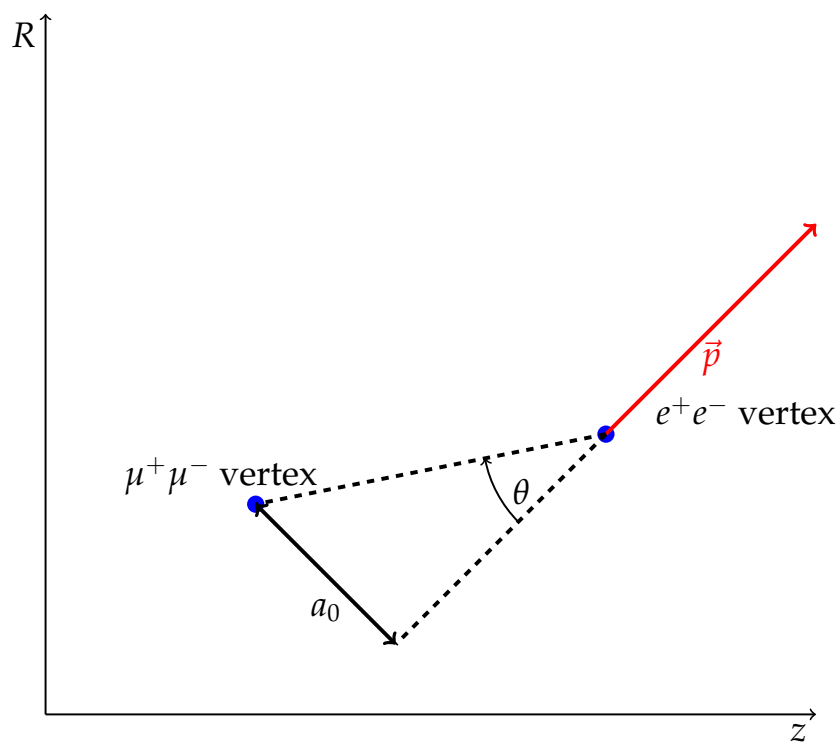

Figure 5.14: A schematic diagram showing the definition of the quantity $a_{0}$ in the $r-z$ plane. The red line represents the momentum vector of the converted photon and the blue points represent the positions of both the $\mu^{+} \mu^{-}$and $e^{+} e^{-}$vertices.

\subsection{Luminosity Measurement}

An accurate measurement of the integrated luminosity of a $p p$ collision data sample is necessary for the measurement of quarkonium (or indeed any final state) production cross sections. A measurement of the integrated luminosity of a data sample, $\int \mathcal{L} d t$, relies upon an accurate measurement of the instantaneous luminosity, $\mathcal{L}$. This can be expressed, for a $p p$ collider, as

$$
\mathcal{L}=\frac{\mu n_{b} f_{r}}{\sigma_{\text {inel }}}
$$

where $\sigma_{\text {inel }}$ is the cross section for inelastic $p p$ interactions, $n_{b}$ is the number of proton bunch pairs which collide in a single revolution, $f_{r}$ is the revolution frequency and $\mu$ is the average number of inelastic $p p$ collisions occurring within each bunch crossing [110]. The ATLAS detector measures the average number of observed inelastic $p p$ interactions in each bunch crossing, $\mu_{\mathrm{vis}}=\epsilon \mu$ and the visible inelastic cross section $\sigma_{\text {vis }}=\epsilon^{\prime} \sigma_{\text {inel }}$ (where $\epsilon^{(\prime)}$ is the efficiency of these measurements) with 
a number of different detectors and algorithms [110]. The quantities $\mu_{\mathrm{vis}}$ and $\sigma_{\mathrm{vis}}$ are typically measured by event or particle counting algorithms using measurements from the ID, calorimeters or the dedicated luminosity measurement detectors, collectively known as LUCID. The LUCID detectors are positioned on either side of the detector $( \pm 17 \mathrm{~m})$ from the IP) and cover the region $5.6<|\eta|<6.0$. The detector consists of sixteen tubes filled with $\mathrm{C}_{4} \mathrm{~F}_{10}$ gas, and is instrumented with photomultiplier tubes designed to detect Cherenkov photons produced when a charged particle passes through the gas [93]. The absolute scale of $\sigma_{\text {vis }}$ measured by the luminosity detector is primarily calibrated by beam-separation scans, also known as van der Meer (vdM) scans [110,111]. This technique allows the absolute luminosity to be directly determined from a combination of the known LHC beam parameters and measurements of the beam widths in the $x$ and $y$ directions. The beam widths are measured by monitoring the activity in the luminosity detectors as the beams are separated (in the $x$ and $y$ directions separately) in steps of known distance [110]. These methods allow the integrated luminosity of the data samples collected during the $\sqrt{s}=7 \mathrm{TeV} p p$ runs of 2010 and 2011 to be measured with a precision of $\pm 3.5 \%$ and $\pm 1.8 \%$ respectively $[110]$. 


\section{Chapter 6}

\section{OBSERVATION OF THE $\chi_{b}$ BOTTOMONIUM STATES}

The following analysis of radiative $\chi_{b}$ decays is published in Ref. [66]. The description of the analysis using unconverted photons is based upon that in [66] but includes some additional details. The complementary analysis using photon conversions, published in the same paper, is also briefly discussed though the analysis was not performed by the author of this thesis.

\subsection{Introduction to the $\chi_{b}$ system}

The $\chi_{b}$ states are the spin triplet $(S=1) P$-wave $(L=1)$ states of the bottomonium system. For a given principal quantum number, $n$, a triplet of $\chi_{b}$ states exists, each state having a different total angular momentum quantum number, $J$. The three states of each $\chi_{b}(n P)$ triplet have total angular momentum, parity and charge conjugation parity quantum numbers $J^{P C}=0^{++}, 1^{++}, 2^{++}$and are denoted $\chi_{b J}(n P)$. The existence of two triplets of $\chi_{b}$ states, the $\chi_{b J}(1 P)$ and $\chi_{b J}(2 P)$ triplets, has been firmly established for many years [112-114]. The dominant decay mode of the $\chi_{b J}(n P)$ states is the radiative transition $\chi_{b J}(n P) \rightarrow \Upsilon(m S) \gamma($ where $m \leq n)$ with branching fractions of $\mathcal{O}(10 \%)$ for the $J=1,2$ states and $\mathcal{O}(1 \%)$ for the $J=0$ states. These large branching fractions suggest that the total transition amplitude is dominated by electric dipole (E1) transitions, though no measurements of the 
multipole compositions have been performed [3]. The world average masses and radiative branching fractions for the $\chi_{b J}(1 P)$ and $\chi_{b J}(2 P)$ states are summarised in Table 6.1. The mass splittings between the individual states within a triplet are small $(\mathcal{O}(10 \mathrm{MeV}))$. The experimental study of the spectroscopy of the $\chi_{b}$ states at $e^{+} e^{-}$colliders running at $\sqrt{s}=m_{\Upsilon(m S)}$ requires fine energy resolution $(\mathcal{O}(1 \mathrm{MeV}))$ to identify the mono-energetic photon lines in $\Upsilon\left(m^{\prime} S\right) \rightarrow \chi_{b J}\left(n^{\prime} P\right) \gamma$ decays (where $\left.m^{\prime}=n^{\prime}+1\right)$. This is also true for hadron colliders, where the unknown initial state requires complete reconstruction of the $\chi_{b J}(n P) \rightarrow \Upsilon(m S) \gamma$ final state and the analysis of an invariant mass spectrum, limiting the potential resolution of hadron collider experiments.

\begin{tabular}{|c|c|c|c|c|}
\hline$n$ & $J$ & Mass $[\mathrm{MeV}]$ & $\mathcal{B}\left(\chi_{b J}(n P) \rightarrow \Upsilon(1 S) \gamma\right)$ & $\mathcal{B}\left(\chi_{b J}(n P) \rightarrow \Upsilon(2 S) \gamma\right)$ \\
\hline \multirow{3}{*}{1} & 0 & $9859.44 \pm 0.42 \pm 0.31$ & $(1.76 \pm 0.35) \%$ & - \\
& 1 & $9892.78 \pm 0.26 \pm 0.31$ & $(33.9 \pm 2.2) \%$ & - \\
& 2 & $9912.21 \pm 0.26 \pm 0.31$ & $(19.1 \pm 1.2) \%$ & $(4.6 \pm 2.1) \%$ \\
& 0 & $10232.5 \pm 0.4 \pm 0.5$ & $(0.9 \pm 0.6) \%$ & $(19.9 \pm 1.9) \%$ \\
2 & 1 & $10255.46 \pm 0.22 \pm 0.50$ & $(9.2 \pm 0.8) \%$ & $(10.6 \pm 2.6) \%$ \\
& 2 & $10268.65 \pm 0.22 \pm 0.50$ & $(7.0 \pm 0.7) \%$ & \\
\hline
\end{tabular}

Table 6.1: Selected properties of the $\chi_{b J}(1 P)$ and $\chi_{b J}(2 P)$ states, with all information taken from [3]. The $\chi_{b J}(n P)$ masses are calculated using the photon energy measured in $\Upsilon(m S) \rightarrow \chi_{b J}(n P) \gamma$ decays (where $\left.m=n+1\right)$. The first uncertainty is associated with the world average value of the photon energy while the second is associated with the uncertainty on the $\Upsilon(m S)$ world average mass.

In principle, many triplets of $\chi_{b}$ states could exist with principal quantum numbers in excess of 2 , though those with masses above the $B \bar{B}$ threshold (10558.52 MeV) are expected to have large hadronic decay widths to $b$ hadron pairs that dwarf the experimentally convenient radiative transitions by orders of magnitude. Additionally, at higher masses, the possibility of mixing between $P$-wave states and higher angular momentum states (or the $B \bar{B}$ continuum, if close to the threshold) becomes more likely, as mass splittings become more compressed and decay widths increase.

However, a third triplet comprising the $\chi_{b J}(3 P)$ states, is generally expected to exist and predictions for the masses of the $\chi_{b J}(3 P)$ states based upon various $Q \bar{Q}$ 
potential models tend to lie below the $B \bar{B}$ threshold (see Table 6.2) [115-117].

\begin{tabular}{|l|c|c|c|c|}
\hline \multirow{2}{*}{ Author(s) } & \multicolumn{4}{|c|}{ Predicted $\chi_{b J}(3 P)$ mass $[\mathrm{MeV}]$} \\
\cline { 2 - 5 } & $J=0$ & $J=1$ & $J=2$ & c.o.g. \\
\hline Kwong \& Rosner (Ref. [116]) & 10500.7 & 10516.0 & 10526.4 & 10520.1 \\
Motyka \& Zalewski (Ref. [117]) & 10503.0 & 10520.1 & 10532.3 & 10525.0 \\
\hline
\end{tabular}

Table 6.2: Selected theoretical predictions for the masses of the $\chi_{b J}(3 P)$ states. The centre of gravity (c.o.g.) of the triplet is the spin averaged mass of the three individual states. The hyperfine splitting between the ${ }^{3} P_{J}$ c.o.g. and the ${ }^{1} P_{1}$ state is expected to be small $(<1 \mathrm{MeV})[14]$. Consequently, the $3^{3} P_{J}$ c.o.g. is expected to coincide closely with the mass of the $3^{1} P_{1} h_{b}(3 P)$ state [117].

The $\chi_{b J}(3 P)$ states are difficult to produce at an $e^{+} e^{-}$collider since the typical production mechanism of $\Upsilon(m S) \rightarrow \chi_{b J}(n P) \gamma$ (where $\left.m=n+1\right)$ decays is highly suppressed for $n=3$, due to the very small $\Upsilon(4 S)$ radiative branching fraction (around $10^{-4}$, assuming $\left.\Gamma\left(\Upsilon(3 S) \rightarrow \chi_{b}(2 P) \gamma\right) \approx \Gamma\left(\Upsilon(4 S) \rightarrow \chi_{b}(3 P) \gamma\right)\right)$. Alternative production modes at $e^{+} e^{-}$colliders include $e^{+} e^{-} \rightarrow e^{+} e^{-} \gamma \gamma \rightarrow e^{+} e^{-} \chi_{b}(3 P)$, though the di-photon transition amplitude is expected to be small (the corresponding values in the $\chi_{c}$ system are $\left.\Gamma\left(\chi_{c 0,2} \rightarrow \gamma \gamma\right) \approx \mathcal{O}(0.1 \mathrm{keV})\right)$. This production mode is only allowed for the $J=0$ and $J=2$ states, since the corresponding amplitude for the $J=1$ states vanishes due to the Landau-Yang theorem $[118,119]$. The $\chi_{b J}(3 P)$ states have not been observed in either of these modes at $e^{+} e^{-}$experiments thus far. Conversely, the potential for observation of the $\chi_{b J}(3 P)$ states at hadron colliders is in principle no worse than for the $\chi_{b J}(1 P)$ and $\chi_{b J}(2 P)$ states. Hadron colliders benefit from the large quarkonium hadro-production cross section but lack the well defined initial state of $e^{+} e^{-}$collider experiments. CDF was the first hadron collider experiment to observe the hadro-production of the $\chi_{b J}(1 P)$ and $\chi_{b J}(2 P)$ states, though no evidence for the $\chi_{b J}(3 P)$ states was observed [65]. However, the early CDF analysis was performed with a very small data sample representing $90 \mathrm{pb}^{-1}$ of integrated luminosity and any potential $\chi_{b J}(3 P)$ signal would have been very difficult to identify given the large statistical uncertainties. This analysis was not updated with the larger data samples collected during Tevatron Run II and repre- 
sented the only published observation of the $\chi_{b}$ states at a hadron collider until the advent of the LHC.

The arrival of the LHC and the large data samples collected by the experiments during LHC Run I provided an opportunity to confirm the CDF observation of $\chi_{b}$ hadro-production and continue the search for the $\chi_{b J}(3 P)$ states. This chapter will discuss the first experimental observation of the $\chi_{b}$ states at the LHC with the ATLAS experiment.

\subsection{Reconstruction of $\chi_{b J}(n P) \rightarrow \Upsilon(m S) \gamma$ decays}

\subsubsection{Data Sample and Event Selection}

The analysis is performed with a sample representing $4.4 \mathrm{fb}^{-1}$ of $\sqrt{s}=7 \mathrm{TeV} p p$ collision data collected by the ATLAS experiment during the 2011 LHC run. Events are only considered for analysis if they were recorded under stable LHC beam conditions with all relevant detector sub-systems fully operational. The data sample used was collected with a selection of single muon and di-muon triggers. The minimum muon transverse momentum $\left(p_{T}^{\mu}\right)$ threshold was $4 \mathrm{GeV}$ for di-muon triggers and $10 \mathrm{GeV}$ for single muon triggers.

\subsubsection{Selection of $\Upsilon(m S) \rightarrow \mu^{+} \mu^{-}$candidates}

The first step towards the reconstruction of $\chi_{b J}(n P) \rightarrow \Upsilon(m S) \gamma$ decays $(m \leq n)$ is the selection of events containing $\Upsilon(m S) \rightarrow \mu^{+} \mu^{-}$candidates. Events are selected that contain two reconstructed muon objects that satisfy the following requirements:

- The two muons are required to have opposite charges

- Each muon must be reconstructed from an inner detector track matched to a track in the muon spectrometer 
- The pseudo-rapidity of each muon must satisfy $\left|\eta^{\mu}\right|<2.3$

- Each muon must have transverse momentum $p_{T}^{\mu}>4 \mathrm{GeV}$

In addition to these requirements, the inner detector track associated with each muon candidate is required to satisfy a series of quality requirements:

- The track should contain at least one pixel B layer (the first layer) hit if such a hit is expected

- The sum of the number of pixel hits and the number of dead pixel sensors crossed by the track must be greater than 1

- The sum of the number of SCT hits and the number of dead SCT sensors crossed by the track must be greater than 5

- The number of crossed pixel and SCT holes (a hole is an expected hit, given the track trajectory, that has not been assigned to the track [120]) must be less than 3

- The track reconstructed in the silicon detectors must have a successful TRT extension if expected

The inner detector tracks of di-muon pairs that satisfy these criteria are then fitted to a common vertex (with no kinematic or mass constraints) and are only retained if the vertex fit result satisfies $\chi^{2} /[$ d.o.f. $]<20$ ([d.o.f.] denotes the number of degrees of freedom in the fit). The remaining di-muon candidates are required to have transverse momentum $p_{T}^{\mu^{+} \mu^{-}}>8 \mathrm{GeV}$ and absolute rapidity $\left|y^{\mu^{+} \mu^{-}}\right|<2.0$. The invariant mass distribution of di-muon candidates satisfying these requirements is shown in Figure 6.1, which exhibits three broad peaks corresponding to the $\Upsilon(1,2,3 S)$ states. Di-muon candidates are considered as $\Upsilon(1 S) \rightarrow \mu^{+} \mu^{-}$candidates if they satisfy $9.25<m\left(\mu^{+} \mu^{-}\right)<9.65 \mathrm{GeV}$ (Region A in Figure 6.1) and $\Upsilon(2 S) \rightarrow \mu^{+} \mu^{-}$candidates if they satisfy $9.80<m\left(\mu^{+} \mu^{-}\right)<10.10 \mathrm{GeV}$ (Region 
B in Figure 6.1). An asymmetric invariant mass selection around the $\Upsilon(2 S)$ peak is chosen to reduce contamination from $\Upsilon(3 S) \rightarrow \mu^{+} \mu^{-}$decays and non-resonant di-muon pairs. This selection is motivated by the fact that $\chi_{b J}(n P) \rightarrow \Upsilon(3 S) \gamma$ decays are kinematically forbidden for $n=1,2$ and the kinematic acceptance for $n=3$ is expected to be negligible.

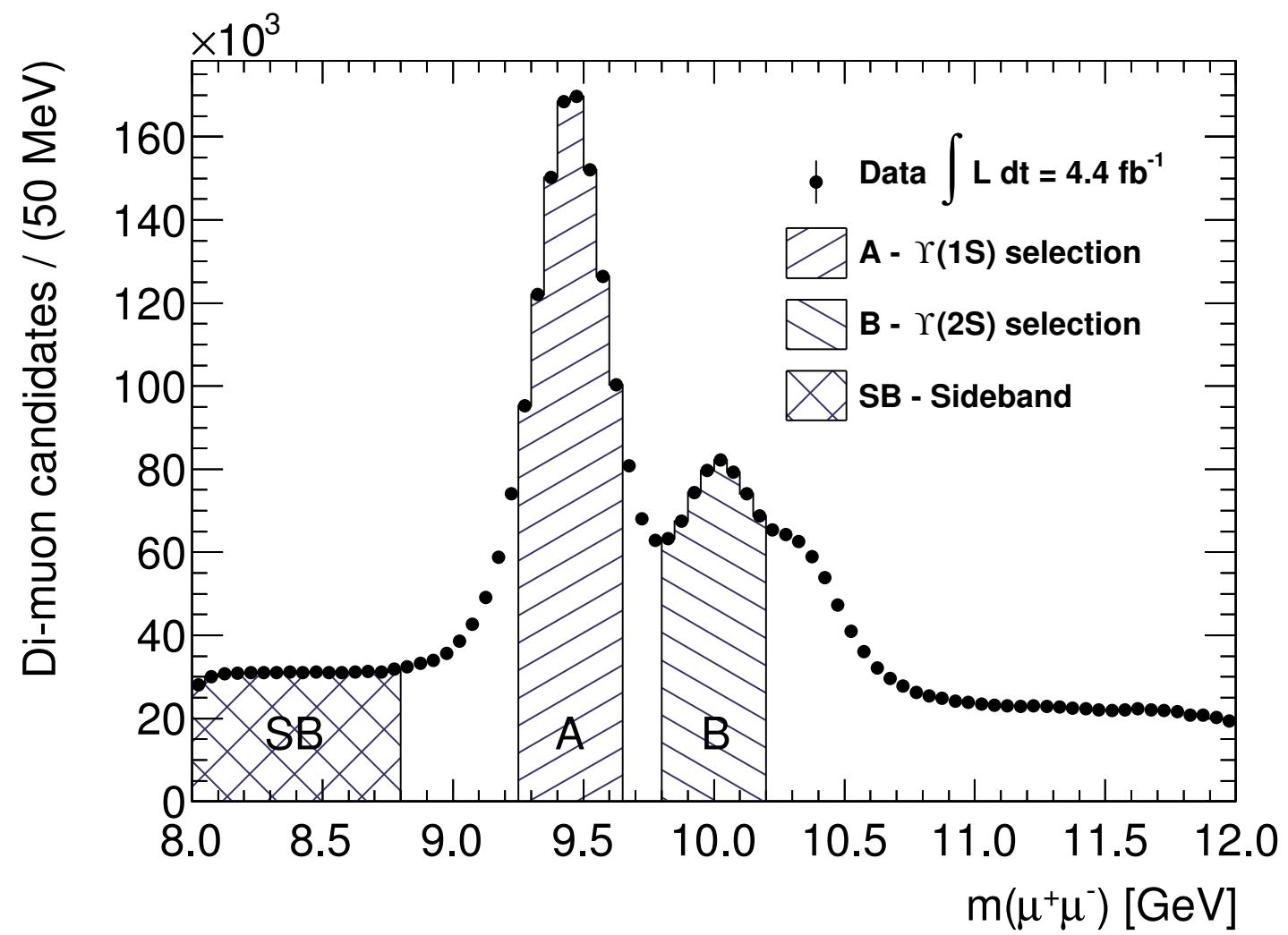

Figure 6.1: The invariant mass $m\left(\mu^{+} \mu^{-}\right)$distribution of $\Upsilon(m S) \rightarrow \mu^{+} \mu^{-}$candidates satisfying the criteria described in Section 6.2.2. Regions A and B show the invariant mass selection criteria for $\Upsilon(1 S) \rightarrow \mu^{+} \mu^{-}$and $\Upsilon(2 S) \rightarrow \mu^{+} \mu^{-}$candidates respectively. The region denoted SB shows the "low mass" sideband.

\subsubsection{Selection of photons from $\chi_{b J}(n P) \rightarrow \Upsilon(m S) \gamma$ decays}

Events containing a reconstructed $\Upsilon(1,2 S) \rightarrow \mu^{+} \mu^{-}$candidate are analysed further to search for photon objects consistent with $\chi_{b J}(n P) \rightarrow \Upsilon(1,2 S) \gamma$ decays. Photons are reconstructed from energy deposits in the EM calorimeter. Unconverted photons with transverse energy $E_{T}^{\gamma}>2.5 \mathrm{GeV}$ and pseudorapidity $\left|\eta^{\gamma}\right|<2.37$ which 
pass the "loose" identification criteria, described in Section 5.4, are retained for further analysis. In addition to these requirements, photons reconstructed within the transition region between the barrel and endcap sections of the EM calorimeter $\left(1.37<\left|\eta^{\gamma}\right|<1.52\right)$ are rejected (since the performance is poorly calibrated due to the complex geometry of the transition).

The direction of the photon momentum is corrected with a calorimeter pointing fitting procedure designed to improve the mass resolution and reject $\mu^{+} \mu^{-} \gamma$ combinations which are inconsistent with having been produced at a single decay vertex. The method used is based upon a technique developed to improve experimental mass resolution in reconstructed $H \rightarrow \gamma \gamma$ decays [121]. The ATLAS EM calorimeter is longitudinally segmented in three layers, allowing photon direction information to be obtained in addition to an energy measurement. The energy deposit barycentres in the first and second layers of the EM calorimeter, in addition to the $\mu^{+} \mu^{-}$vertex, provide three points in the $r-z$ plane of the detector, from which to extract a more precise photon $\eta$ measurement. These three points are fitted with a straight photon trajectory using a simple $\chi^{2}$ minimisation procedure to yield a corrected value of the polar angle $\theta$, which in turn is used to calculate a corrected value of $\eta$. Fits to $\mu^{+} \mu^{-} \gamma$ candidates which converge with $\chi^{2} /[$ d.o.f. $]<200$ are considered successful and the corrected photon momentum is adopted, while all other candidates are rejected. The reconstructed energy and azimuthal angle of the photon are not altered in this procedure. Reconstructed $\mu^{+} \mu^{-} \gamma$ combinations which are successfully corrected, in addition to passing all the requirements described above, are considered to be candidate $\chi_{b J}(n P) \rightarrow \Upsilon(1,2 S) \gamma$ decays. If multiple unique $\Upsilon(1,2 S) \gamma$ candidates are selected within a single event, all candidates are retained (though no events are found with more than one $\Upsilon(1,2 S) \gamma$ candidate in the dataset used in this analysis). 


\subsection{Observation of the $\chi_{b}$ states}

The mass difference distribution $m\left(\mu^{+} \mu^{-} \gamma\right)-m\left(\mu^{+} \mu^{-}\right)+m_{\Upsilon(1 S)}$ (where $m_{\Upsilon(1 S)}$ is the world average value for the mass of the $\Upsilon(1 S)$ state [3]) for selected $\chi_{b J}(n P) \rightarrow$ $\Upsilon(1 S) \gamma$ candidates is shown in Figure 6.2. The mass difference distribution is analysed in favour of the three-body invariant mass $m\left(\mu^{+} \mu^{-} \gamma\right)$ as it offers improved mass resolution due to a partial cancellation in the experimental di-muon mass resolution that contributes to both $m\left(\mu^{+} \mu^{-} \gamma\right)$ and $m\left(\mu^{+} \mu^{-}\right)$. The distribution exhibits two clear peaks in the regions around $9.9 \mathrm{GeV}$ and $10.25 \mathrm{GeV}$ that are consistent with the masses of the $\chi_{b}(1 P)$ and $\chi_{b}(2 P)$ states. The experimental mass resolution is insufficient to resolve the individual $\chi_{b J}$ states that together form the observed peaks. In addition to these known structures, a further peaking structure is observed at a mass of around $10.55 \mathrm{GeV}$. The mass of the additional structure is not compatible with any previously observed bottomonium state that could decay to $\Upsilon(1 S) \gamma[3]$. Given the close proximity of this structure to the predicted mass centre of gravity for the $\chi_{b}(3 P)$ states shown in Table 6.2, the additional structure is provisionally identified as a candidate for the previously unseen $\chi_{b}(3 P)$ states.

The general shape of the background contribution to the mass difference distribution can be estimated from events which fail the $\Upsilon(n S)$ mass requirements but which satisfy all other selection requirements (though some care must be taken in placing too much trust in this method, as will be discussed in Section 6.6). $\mu^{+} \mu^{-} \gamma$ candidates which pass all of the selection but which have a di-muon invariant satisfying $8.0<m\left(\mu^{+} \mu^{-}\right)<8.8 \mathrm{GeV}$, the "low mass" sideband of the $\Upsilon(n S)$ peaks, are used to provide an estimate of the shape of the background contribution, as shown in the shaded regions of Figure 6.2.

The mass difference distribution $m\left(\mu^{+} \mu^{-} \gamma\right)-m\left(\mu^{+} \mu^{-}\right)+m_{\Upsilon(2 S)}\left(\right.$ where $m_{\Upsilon(2 S)}$ is the world average value for the mass of the $\Upsilon(2 S)$ state [3]), shown in Figure 6.3, is also analysed to search for evidence of $\chi_{b J}(n P) \rightarrow \Upsilon(2 S) \gamma$ decays. Some small 
excess of events above the background expectation (assuming the normalised sideband distribution is a good approximation to the background) is observed in the region around $10.55 \mathrm{GeV}$. While the mass of this structure is broadly compatible with the $\chi_{b}(3 P)$ candidate observed in the $\Upsilon(1 S) \gamma$ final state, it is not found to be statistically significant. 

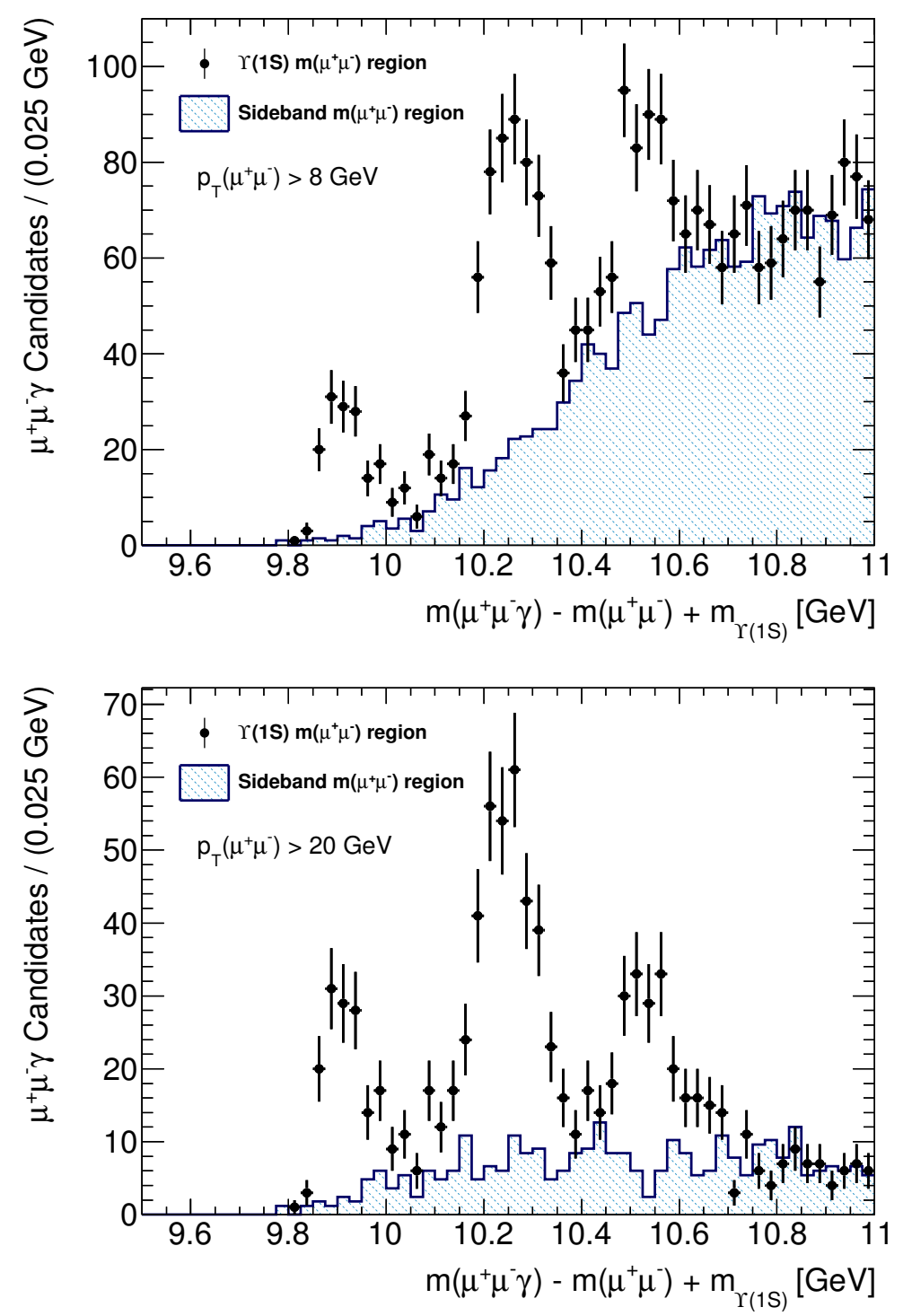

Figure 6.2: The $m\left(\mu^{+} \mu^{-} \gamma\right)-m\left(\mu^{+} \mu^{-}\right)+m_{\Upsilon(1 S)}$ distributions for $\chi_{b}(n P) \rightarrow \Upsilon(1 S) \gamma$ candidates reconstructed with unconverted photons. The same distributions for $\mu^{+} \mu^{-} \gamma$ candidates with a di-muon invariant mass within the "low mass" sideband region is overlaid and normalised to the same number of events as the $\chi_{b}$ candidate distribution in the region above $10.7 \mathrm{GeV}$. The $\mu^{+} \mu^{-} \gamma$ candidates in the lower plot are required to have a di-muon transverse momentum $p_{T}^{\mu^{+} \mu^{-}}>20 \mathrm{GeV}$. 

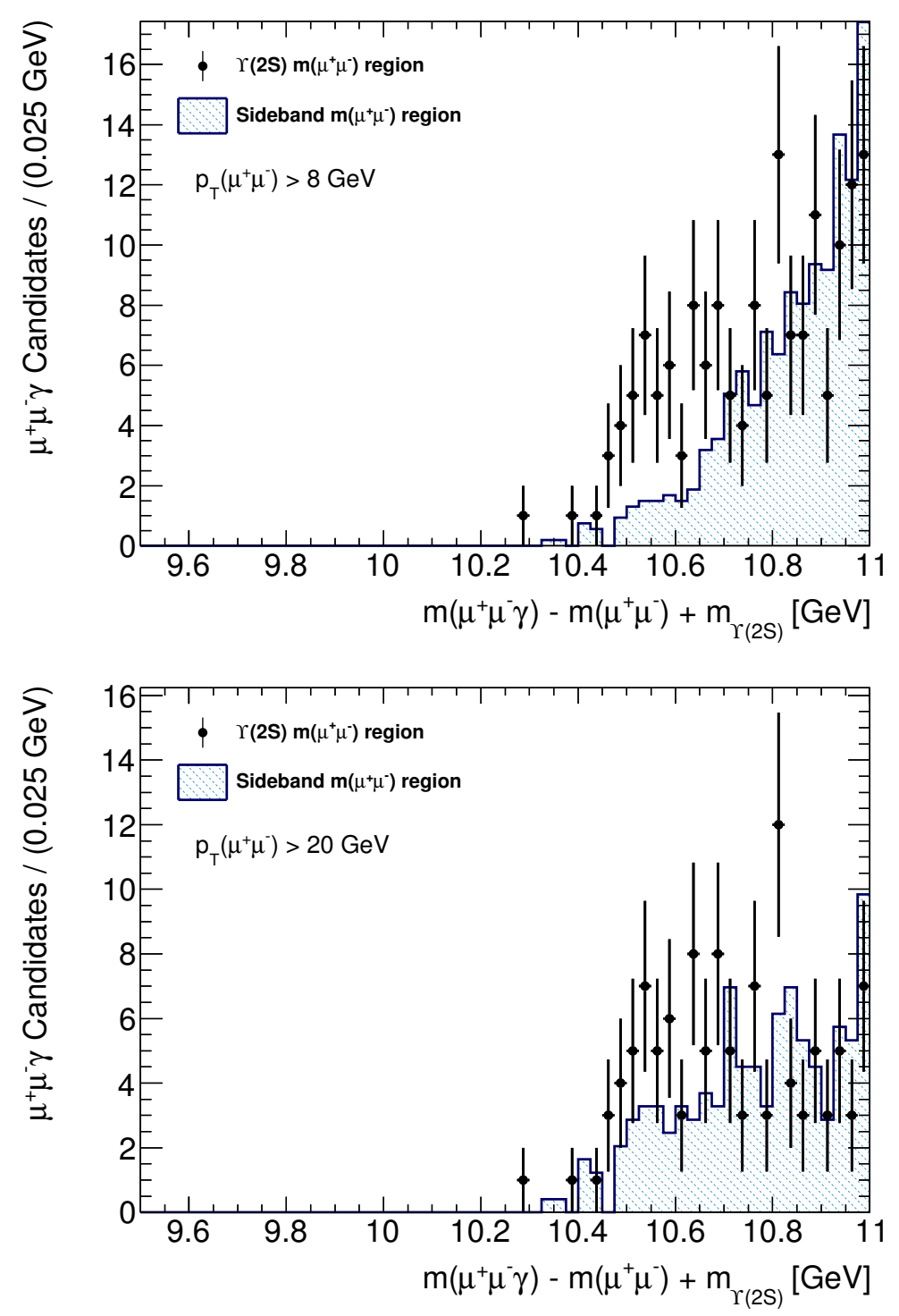

Figure 6.3: The $m\left(\mu^{+} \mu^{-} \gamma\right)-m\left(\mu^{+} \mu^{-}\right)+m_{\Upsilon(2 S)}$ distributions for $\chi_{b}(n P) \rightarrow \Upsilon(2 S) \gamma$ candidates reconstructed with unconverted photons. The same distributions for $\mu^{+} \mu^{-} \gamma$ candidates with a di-muon invariant mass within the "low mass" sideband region is overlaid and normalised to the same number of events as the $\chi_{b}$ candidate distribution in the region above $10.7 \mathrm{GeV}$. The $\mu^{+} \mu^{-} \gamma$ candidates in the lower plot are required to have a di-muon transverse momentum $p_{T}^{\mu^{+} \mu^{-}}>20 \mathrm{GeV}$. 


\subsection{Cross Checks}

Several checks are performed to ensure that the structure interpreted as a candidate for the $\chi_{b}(3 P)$ states is due to the genuine decays of a physical mass state (or states) and not a spurious experimental effect.

- $m\left(\mu^{+} \mu^{-}\right)$Sideband Studies: The mass difference distribution $m\left(\mu^{+} \mu^{-} \gamma\right)-$ $m\left(\mu^{+} \mu^{-}\right)$for $\mu^{+} \mu^{-} \gamma$ candidates with di-muon invariant masses outside the $\Upsilon(n S)$ peak region are analysed to search for any structures similar to the $\chi_{b}(3 P)$ candidate peak. The mass difference distributions for $\mu^{+} \mu^{-} \gamma$ candidates with di-muon invariant masses within both the low mass sidebands $\left(8.0<m\left(\mu^{+} \mu^{-}\right)<8.8 \mathrm{GeV}\right)$ and high mass $\left(10.7<m\left(\mu^{+} \mu^{-}\right)<12.0 \mathrm{GeV}\right)$ sidebands are investigated. No evidence for any peaking structure is observed in either mass difference distribution, as shown in Figure 6.2.

- Event Mixing: Events containing $\Upsilon(1 S)$ candidates are combined with photon candidates which pass the photon selection criteria but which are reconstructed in an independent $p p$ bunch crossing event. The approach involves running the analysis selection on the full data sample but with all photon information taken from the previous event. The mass difference distribution for a sample of data events mixed in this way is shown in Figure 6.4. The mass difference distribution for this sample of "mixed" $\Upsilon(1 S) \gamma$ pairs does not exhibit any clear peaking structures in the $\chi_{b}$ mass region, as shown in Figure 6.4.

- Muon Photon Overlap: Events in which one or both muons from the $\Upsilon(1 S) \rightarrow$ $\mu^{+} \mu^{-}$decay are spatially close to the unconverted photon candidate could affect the energy measurement of the photon, due to possible additional energy deposits in the same EM cluster. The photon cluster size is $3 \times 5$ cells $=$ $0.075(\eta) \times 0.125(\phi)$ in the barrel region of the EM calorimeter and $5 \times 5$ cells $=$ $0.125(\eta) \times 0.125(\phi)$ in the endcap region. If a muon is within $\Delta R(\mu, \gamma)<$ 
$0.15(0.18)\left(\right.$ where $\Delta R=\sqrt{(\Delta \phi)^{2}+(\Delta \eta)^{2}}$ ) of a photon reconstructed within the barrel (endcap) there is a possibility that it will deposit some energy (typically around $300 \mathrm{MeV}$ ) within the EM calorimeter cells that contribute to the measurement of the photon cluster energy. To check whether this possible correlated mis-measurement could be responsible for the structure interpreted as a $\chi_{b}(3 P)$ candidate, the full sample of $\chi_{b}(n P) \rightarrow \Upsilon(1 S) \gamma$ candidates is split into subsets based on the spatial separation between the unconverted photon candidate and the closest muon, $\Delta R(\mu, \gamma)$. Only around $10 \%$ of the $\mu^{+} \mu^{-} \gamma$ candidates are reconstructed with $\Delta R(\mu, \gamma)<0.15(0.18)$, as shown in Figure 6.5. The $\mu^{+} \mu^{-} \gamma$ candidates within the $\chi_{b}(n P)$ signal peaks in the $m\left(\mu^{+} \mu^{-} \gamma\right)-m\left(\mu^{+} \mu^{-}\right)+m_{\Upsilon(1 S)}$ distribution are found to have no strong dependence on $\Delta R(\mu, \gamma)$.

- Time Dependent Effects and Pileup: The full sample of $\chi_{b}(n P) \rightarrow \Upsilon(1 S) \gamma$ candidates is split into two subsets based on the data acquisition system run number (i.e. time of data collection) to check for any possible time dependence of the structures in the mass difference distribution due to changes to the LHC or detector running conditions. In particular, the instantaneous luminosity delivered to ATLAS by the LHC increased significantly throughout the 2011 run. One consequence of this was an increase in the number of $p p$ interactions in each bunch crossing (pileup) as a function of time. Additionally, the selection applied by the muon trigger algorithms used to collect the 2011 data sample evolved slightly during the run to account for the increasing instantaneous luminosity. To check whether any of these time dependent changes to the experimental conditions could have resulted in systematic changes to the mass difference distributions, two data subsets ("early" and "late") are defined based upon their run number. The same three peaking structures and the background shape are observed in both data subsets, suggesting that the changes in the experimental conditions are largely inconsequential to the shape of the 
mass difference distribution.

- MC Simulation Samples: The analysis selection is performed on a sample of simulated $p p \rightarrow \Upsilon(1 S) X$ events generated with the PYTHIA 6 MC generator [99]. The simulated events are propagated through the ATLAS detector simulation and processed with the same reconstruction algorithms used to process the data. The simulated event samples contain $\chi_{b}(1,2 P) \rightarrow \Upsilon(1 S) \gamma$ decays but do not contain decays of any other bottomonium states to $\Upsilon(1 S) \gamma$ final states. The mass difference distributions obtained from these simulation samples exhibits a $\chi_{b}(1 P)$ signal peak qualitatively similar to that observed in data but does not exhibit any structures in the region of the $\chi_{b}(3 P)$ candidate peak observed in data, as shown in Figure 6.6. The sample does not include any $\chi_{b}(2 P) \rightarrow \Upsilon(1 S) \gamma$ decays due to the limitations of the PYTHIA implementation of bottomonium production processes.

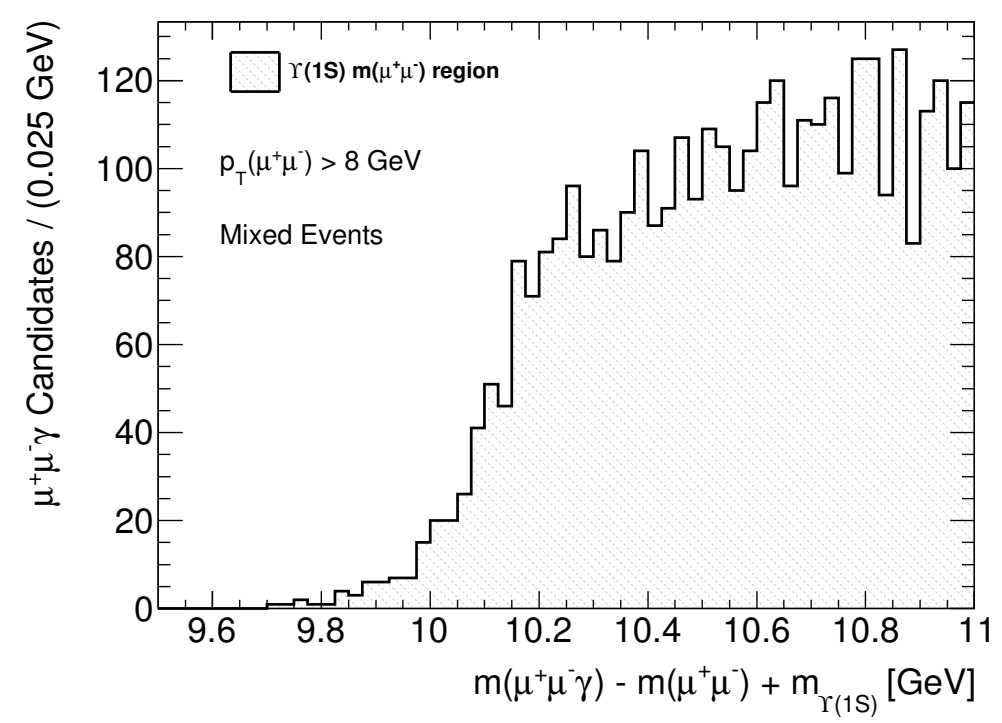

Figure 6.4: The mass difference $m\left(\mu^{+} \mu^{-} \gamma\right)-m\left(\mu^{+} \mu^{-}\right)+m_{\Upsilon(1 S)}$ distribution for a sample of mixed data events. This sample is built by associating data events which satisfy the $\Upsilon(1 S)$ selection with photon information from the previous event. 


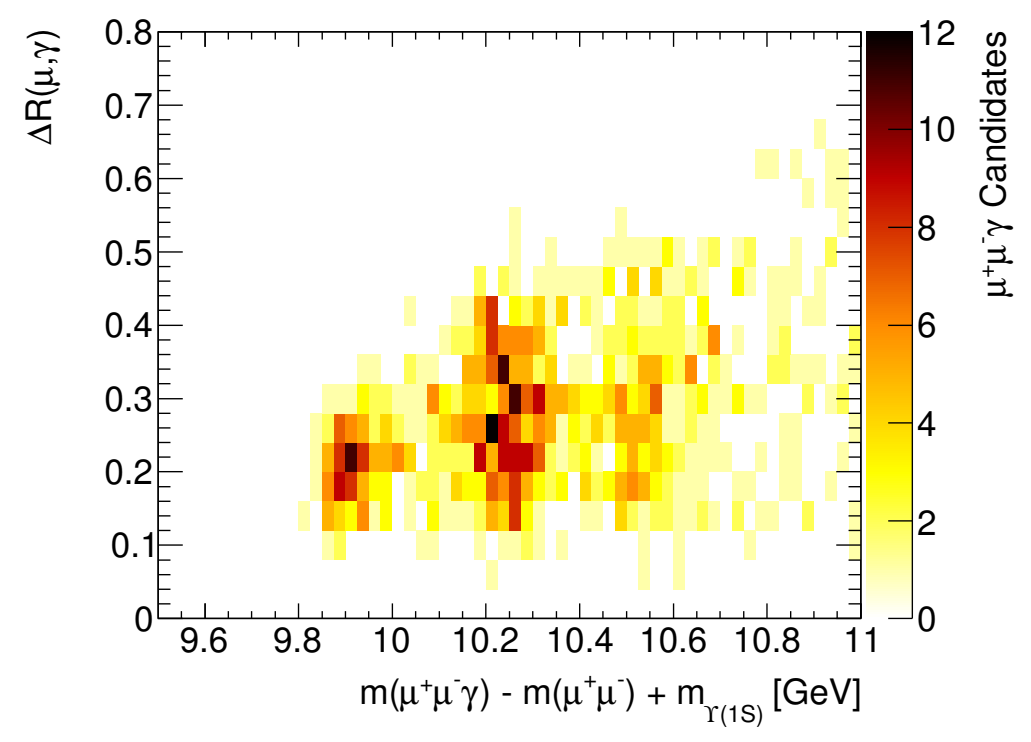

Figure 6.5: The spatial separation $\Delta R(\mu, \gamma)$, versus the mass difference $m\left(\mu^{+} \mu^{-} \gamma\right)-$ $m\left(\mu^{+} \mu^{-}\right)+m_{\Upsilon(1 S)}$ distribution for selected $\chi_{b}(n P) \rightarrow \Upsilon(1 S) \gamma$ candidates. The distribution shows that the $\mu^{+} \mu^{-} \gamma$ candidates within the $\chi_{b}(n P)$ signal peaks in the $m\left(\mu^{+} \mu^{-} \gamma\right)-m\left(\mu^{+} \mu^{-}\right)+m_{\Upsilon(1 S)}$ distribution are distributed with no strong dependence on $\Delta R(\mu, \gamma)$.

\subsection{Fitting Procedure}

Before the fitting procedure is performed, an additional requirement of $p_{T}^{\mu^{+} \mu^{-}}>$ $20 \mathrm{GeV}$ is imposed on the selected $\chi_{b}(n P) \rightarrow \Upsilon(1 S) \gamma$ candidates, which enhances the statistical significance of the $\chi_{b}(1 P)$ and $\chi_{b}(2 P)$ mass peaks (irrespective of the $\chi_{b}(3 P)$ candidate peak), as shown in Figure 6.2 .

An unbinned maximum likelihood fit is performed to the $m\left(\mu^{+} \mu^{-} \gamma\right)-m\left(\mu^{+} \mu^{-}\right)+$ $m_{\Upsilon(1 S)}$ distribution of selected $\chi_{b}(n P) \rightarrow \Upsilon(1 S) \gamma$ candidates to measure the experimental "mass barycentre" of each $\chi_{b}(n P)$ peak. The experimental mass resolution is considerably larger than the known $\chi_{b}(1 P)$ and $\chi_{b}(2 P)$ mass splittings. The observed $\chi_{b}(1 P)$ and $\chi_{b}(2 P)$ mass peaks represent mixtures of each of the two triplets of $\chi_{b J}$ states, each weighted by the product of its relative production rate, radiative branching fraction and total experimental acceptance. This complicated production-averaged mass is referred to as the experimental mass barycentre. While the branching fractions for the individual $\chi_{b J}$ states are known for the $\chi_{b}(1 P)$ and 


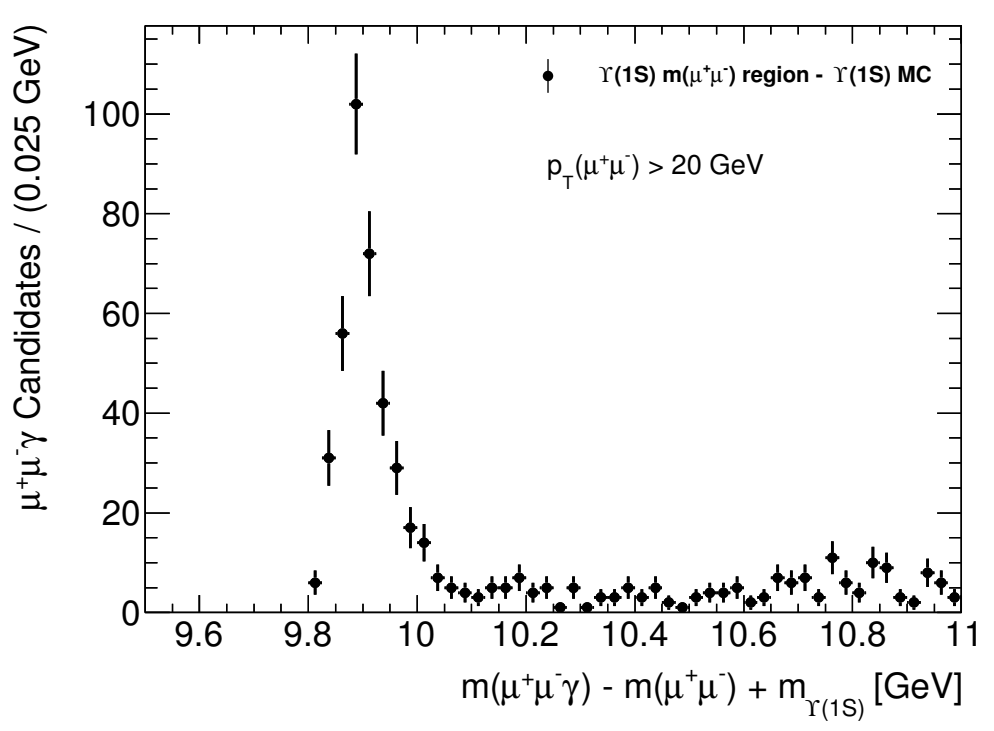

Figure 6.6: The mass difference $m\left(\mu^{+} \mu^{-} \gamma\right)-m\left(\mu^{+} \mu^{-}\right)+m_{\Upsilon(1 S)}$ distribution for a sample of simulated $\chi_{b}(1 P) \rightarrow \Upsilon(1 S) \gamma$ events. The peak in the distribution corresponds to $\chi_{b}(1 P) \rightarrow \Upsilon(1 S) \gamma$. Neglecting this mass peak, no further mass peaks similar to those observed in data are present.

$\chi_{b}(2 P)$ triplets, the production cross sections for the states have not been measured at the LHC. It is therefore not possible to attempt to fit the $\chi_{b}(1 P)$ and $\chi_{b}(2 P)$ peaks reliably with a probability density function (PDF) composed of three individual contributions, given the poor mass resolution. The situation is complicated further in the case of the $\chi_{b}(3 P)$ candidate by a lack of knowledge of the presence or attributes of any fine structure. Given the absence of the information necessary to measure any physically meaningful masses, the experimental mass barycentres are measured directly. While these quantities are dependent on the experimental detection methods they can still be used to make a qualitative and indirect comparison with the known $\chi_{b J}(1,2 P)$ masses and the theoretical predictions for the masses of the $\chi_{b J}(3 P)$ states.

The mass difference distribution $\tilde{m}=m\left(\mu^{+} \mu^{-} \gamma\right)-m\left(\mu^{+} \mu^{-}\right)+m_{\Upsilon(1 S)}$ is modelled with the PDF

$$
P(\tilde{m} ; \Psi)=\sum_{n=1}^{3} f_{n} \cdot P_{\mathrm{sig}}^{n}\left(\tilde{m} ; \Phi_{n}\right)+f_{\mathrm{bkgd}} \cdot P_{\mathrm{bkgd}}(\tilde{m} ; \Theta)
$$


where $P_{\text {sig }}$ is the PDF describing each $\chi_{b}(n P)$ peak and $P_{\mathrm{bkgd}}$ is the PDF describing the background contribution to the distribution. $\Psi, \Phi_{n}$ and $\Theta$ are sets of free parameters $\left(\Psi \in\left\{\Phi_{n}, \Theta\right\}\right)$ and the constraint $f_{1}+f_{2}+f_{3}+f_{\mathrm{bkgd}}=1$ is imposed. A single Gaussian signal PDF is chosen, motivated by the fact that the experimental resolution (which is well described by a Gaussian resolution function) in the mass difference distribution (around $45 \mathrm{MeV}$ ) is significantly greater than the mass splitting between the $J=1$ and $J=2$ states in each triplet $\left(19 \mathrm{MeV}\right.$ for the $\chi_{b}(1 P)$ triplet and $14 \mathrm{MeV}$ for the $\chi_{b}(2 P)$ triplet). Contributions from the $J=0$ states of the triplets are expected to be small given their much lower branching fractions to $\Upsilon(1 S) \gamma$, relative to the $J=1$ and $J=2$ states. The choice of a Gaussian PDF is further motivated by the lack of knowledge of any potential unresolved substructure within the $\chi_{b}(3 P)$ candidate peak.

Each of the three $\chi_{b}$ signal peaks present in the $\tilde{m}$ distribution is modelled with a Gaussian PDF, each with a free mean value (an estimate of the experimental mass barycentre) $\bar{m}_{n}$ and width $\sigma_{n}$, denoted by $P_{\text {sig. }}^{n}\left(\tilde{m} ; \bar{m}_{n}, \sigma_{n}\right)$. The background PDF is modelled with the empirically motivated distribution,

$$
P_{\mathrm{bkgd}}(\tilde{m} ; \alpha, \beta)=\exp \left(\alpha \cdot \tilde{m}+\beta \cdot \tilde{m}^{-2}\right)
$$

where $\alpha$ and $\beta$ are both free parameters. The analytical form of the background PDF is chosen to imitate the shape of the $m\left(\mu^{+} \mu^{-} \gamma\right)-m\left(\mu^{+} \mu^{-}\right)$distribution for $\mu^{+} \mu^{-} \gamma$ candidates with a di-muon invariant mass in the sidebands of the $m\left(\mu^{+} \mu^{-}\right)$ distribution. The result of the fit is shown in Figure 6.7. In addition to the nominal fit, performed with the requirement $p_{T}^{\mu^{+} \mu^{-}}>20 \mathrm{GeV}$ imposed, Figure 6.7 also shows a fit to the mass difference distributions with a relaxed requirement of $p_{T}^{\mu^{+} \mu^{-}}>$ $8 \mathrm{GeV}$. The $\chi_{b}$ signal to background ratio with the relaxed $p_{T}^{\mu^{+} \mu^{-}}$requirement is significantly lower than for the nominal selection. 

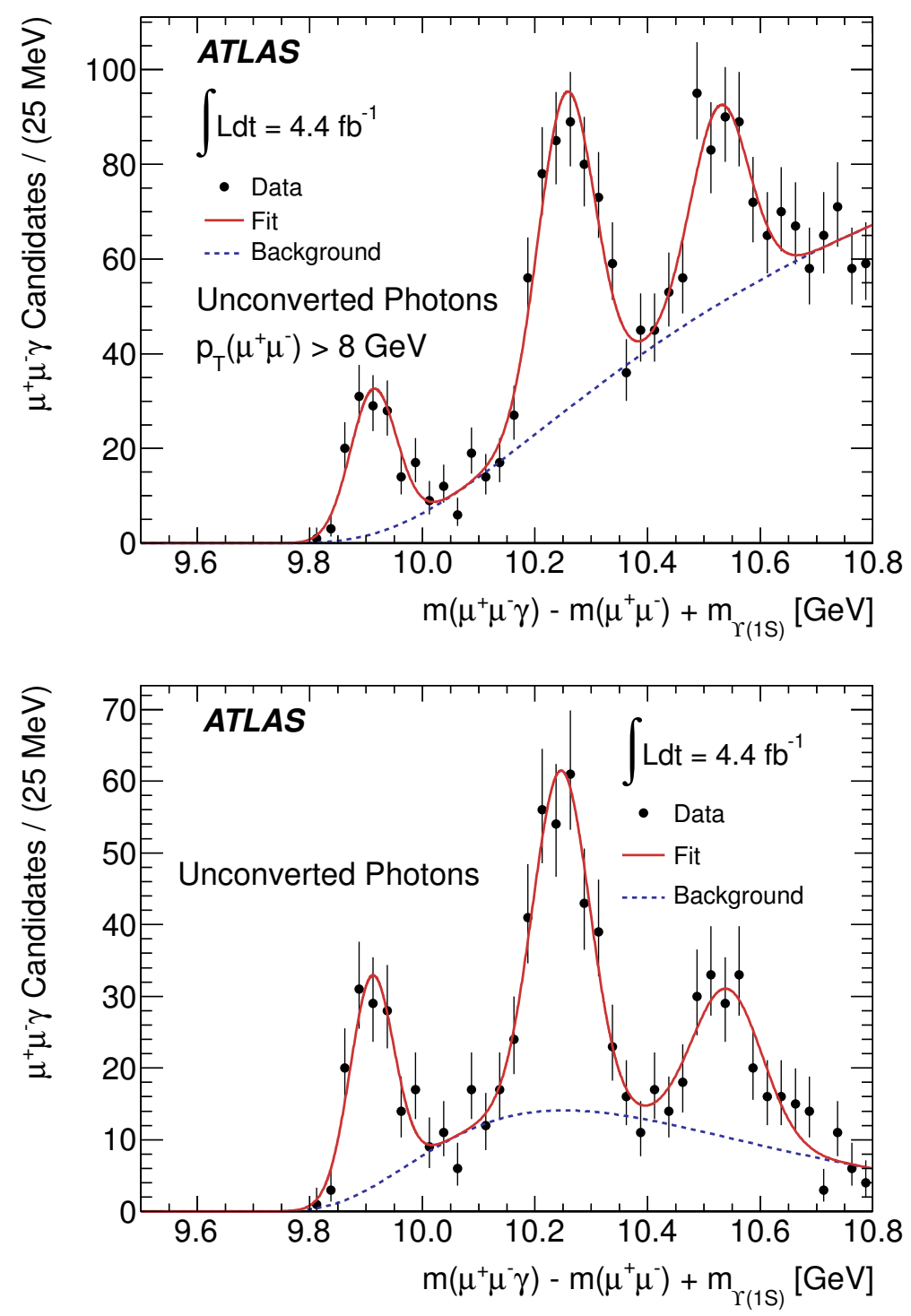

Figure 6.7: The results of unbinned maximum likelihood fits to the $m\left(\mu^{+} \mu^{-} \gamma\right)-$ $m\left(\mu^{+} \mu^{-}\right)+m_{\Upsilon(1 S)}$ distribution for $\chi_{b}(n P) \rightarrow \Upsilon(1 S) \gamma$ candidates reconstructed with unconverted photons. The requirement $p_{T}^{\mu^{+} \mu^{-}}>8 \mathrm{GeV}$ is imposed in the upper figure while $p_{T}^{\mu^{+} \mu^{-}}>20 \mathrm{GeV}$ is imposed in the lower figure. The fit result shown in the lower figure is used to measure experimental mass barycentres of the $\chi_{b}$ signals. 


\subsection{Systematic Uncertainties}

Several sources of systematic uncertainty are studied that could affect the measurements of the experimental mass barycentres of the $\chi_{b}$ signals:

- Choice of $\chi_{b}$ signal PDF: To check that the choice of a single Gaussian PDF to model each $\chi_{b}$ peak is justified, given the known mass resolution and mass splittings, an alternative signal fit model is tested. The signal PDF for each $\chi_{b}$ peak is modified to the sum of two Gaussian PDFs. The mean values of the two Gaussians, $\bar{m}_{n}^{J=1}$ and $\bar{m}_{n}^{J=2}$ are constrained such that $\bar{m}_{n}^{J=2}-\bar{m}_{n}^{J=1}=$ $\Delta m_{21}(n P)$ where $\Delta m_{21}(n P)$ is the mass splitting between the $\chi_{b 1}(n P)$ and $\chi_{b 2}(n P)$ states. In the case of the $\chi_{b}(1 P)$ and $\chi_{b}(2 P)$ triplets, this information is taken from the world average values [3], while a theoretical prediction of $\Delta m_{21}(3 P)=12 \mathrm{MeV}$ is used for the $\chi_{b}(3 P)$ triplet [117]. The widths of the two Gaussian PDFs are constrained to the same value while the relative normalisation of the two Gaussian PDFs is included as an additional free parameter (common to all three signal peaks). The changes in the peak positions with this alternative signal model, compared to the nominal fit, are found to be within $\pm 3 \mathrm{MeV}$ for each peak, substantially lower than the statistical uncertainty. This suggests that the data have no sensitivity to the fine structure and the use of a single Gaussian PDF is well motivated. No systematic uncertainty on the measured experimental mass barycentres was assigned due to the modelling of the $\chi_{b}$ signal in the fit model.

- Choice of background PDF: An accurate measurement of the experimental mass barycentres relies on an accurate modelling of the background contribution to mass difference distribution. In addition to describing the shape of the background accurately, the parameterisation of the background PDF must not introduce any bias to the fit result due to strong parameter correlations. 
To quantify the systematic uncertainty due to the choice of background PDF, several alternative models are tested:

1. Simultaneous fit to sideband data: The mass difference distribution of $\mu^{+} \mu^{-} \gamma$ candidates with $m\left(\mu^{+} \mu^{-}\right)$outside the $\Upsilon(m S)$ peaks provides an estimate of the shape of the background contribution to the mass difference distribution of the $\chi_{b}(n P)$ candidates. Differences between the two distributions can arise due the differing muon kinematics (generally due to differing $\left.m\left(\mu^{+} \mu^{-}\right)\right)$and the contributions to the background distribution from resonant sources. To minimise the differences in shape due to muon kinematics, only the "low mass" sideband $\left(7.0<m\left(\mu^{+} \mu^{-}\right)<8.8 \mathrm{GeV}\right)$ is used, as it is closer to the $\Upsilon(1 S)$ mass $(9.46 \mathrm{GeV})$ than the "high mass" sideband. Decay chains such as $\Upsilon(2,3 S) \rightarrow \Upsilon(1 S) \pi^{0} \pi^{0}$ (with $\pi^{0} \rightarrow \gamma \gamma$, where $\Upsilon(1 S) \rightarrow \mu^{+} \mu^{-}$and a single photon are reconstructed) and $\Upsilon(2,3 S) \rightarrow \chi_{b}(1,2 P) \gamma_{1} \rightarrow \Upsilon(1 S) \gamma_{1} \gamma_{2}$ (where the reconstructed $\Upsilon(1 S)$ and $\gamma_{1}$ are paired) represent the dominant sources of such resonant backgrounds. The shapes and rates of these backgrounds relative to the $\chi_{b}(1 P)$ and $\chi_{b}(2 P)$ signals are shown in Figure 6.10. These contributions have a rate significantly below the $\chi_{b}(1,2 P)$ signals and below the total relative background when compared to Figure 6.7. This suggests that the use of the "low mass" sideband distribution is a reasonable approximation to the background contribution to the mass difference distribution. The fitting procedure is modified to simultaneously fit the nominal background PDF to both the "low mass" sideband mass difference distribution in addition to the mass difference distribution of the $\chi_{b}$ candidates. The nominal fit is also performed with the background parameters $\alpha$ and $\beta$ fixed to the values obtained in a fit to the "low mass" sideband mass difference distribution.

2. Alternative background PDFs: Two alternative background PDFs are also 
tested. The first is the PDF $P_{\text {alt. }}^{1}(\tilde{m} ; \alpha, \beta)=m^{\alpha} \exp (\beta \cdot \tilde{m})$ which has a shape similar to the nominal background PDF and has the same number of free parameters. The second alternative PDF is a modified Novosibirsk function [122] that contains four free parameters. Additionally, the "low mass" sideband distribution is used to form a non-parametric Gaussian kernel estimation PDF [123] which is also tested as an alternative background PDF, as shown in Figure 6.8.

The maximum single deviation from the nominal fitted experimental mass barycentres from all of the alternative models is assigned as a systematic uncertainty due to modelling of the background contribution to the mass difference distribution. In this way, the systematic uncertainty on the measured experimental mass barycentres due to modelling of the background is estimated at $\pm 6 \mathrm{MeV}, \pm 8 \mathrm{MeV}$ and $\pm 21 \mathrm{MeV}$ for the $\chi_{b}(1 P), \chi_{b}(2 P)$ and $\chi_{b}(3 P)$ signals respectively.

- Photon Energy Scale: The accuracy of the measurement of the photon energy in $\chi_{b}(n P) \rightarrow \Upsilon(1 S) \gamma$ decays has a direct impact on the measured experimental mass barycentres of the $\chi_{b}$ signals. The positions of the $\chi_{b}(n P)$ peaks in the mass difference $m\left(\mu^{+} \mu^{-} \gamma\right)-m\left(\mu^{+} \mu^{-}\right)$distribution are strongly correlated to the measured photon energy. The uncertainty on the unconverted photon energy scale is estimated at $\pm 2 \%$, from measurements of $Z \rightarrow e^{+} e^{-}$and $J / \psi \rightarrow e^{+} e^{-}$decays in data [124]. To estimate the effect of this energy scale uncertainty, the measured energy of each photon is scaled by $\pm 2 \%$ and the mass difference distribution is refitted. The result is a shift in the measured experimental mass barycentre of each $\chi_{b}$ peak by $\pm 2 \%$ (as expected given the direct correlation between the photon energy and the peak position in $\left.m\left(\mu^{+} \mu^{-} \gamma\right)-m\left(\mu^{+} \mu^{-}\right)\right)$. The systematic uncertainties on the measured experimental mass barycentres due to the uncertainty on the photon energy scale 
are estimated at $\pm 9 \mathrm{MeV}, \pm 16 \mathrm{MeV}$ and $\pm 22 \mathrm{MeV}$ for the $\chi_{b}(1 P), \chi_{b}(2 P)$ and $\chi_{b}(3 P)$ signals respectively.

- Muon Photon Overlap: The fit procedure is repeated with the requirement $\Delta R(\mu, \gamma)>0.15(0.18)$ for photons reconstructed in the barrel (endcap) region of the EM calorimeter, to exclude $\chi_{b}$ candidates reconstructed from photons whose energy measurement might have been biased by energy deposits from a spatially close muon. No significant change in the measured mass barycentres of the $\chi_{b}$ signals is observed and no systematic uncertainty is assigned.

- Photon Identification Energy Scale Bias: The photon identification requirements of the "loose" selection described in Section 5.4 are tuned for photons with $p_{T}>15 \mathrm{GeV}$. To ensure that these requirements do not cause an unintentional bias in the calibrated energy scale for much softer photons, the analysis and fitting procedure are repeated without the "loose" photon identification requirement. The $m\left(\mu^{+} \mu^{-} \gamma\right)-m\left(\mu^{+} \mu^{-}\right)+m_{\Upsilon(1 S)}$ distribution for $\chi_{b}$ candidates selected without the "loose" requirement is shown in Figure 6.9. The distribution exhibits a significantly larger background contribution, though the qualitative position and prominence of the peaks is relatively unchanged. This $m\left(\mu^{+} \mu^{-} \gamma\right)-m\left(\mu^{+} \mu^{-}\right)+m_{\Upsilon(1 S)}$ distribution is fitted with the nominal fitting procedure but with a background PDF derived by applying the Gaussian kernel estimation procedure to the "low mass" sideband data, as discussed earlier [123]. No significant change in the fitted values of the measured experimental mass barycentres is observed suggesting that the "loose" selection does not significantly bias the energy calibration of low $p_{T}$ photons. No additional systematic uncertainty on the photon energy scale, beyond that discussed earlier, is assigned. 


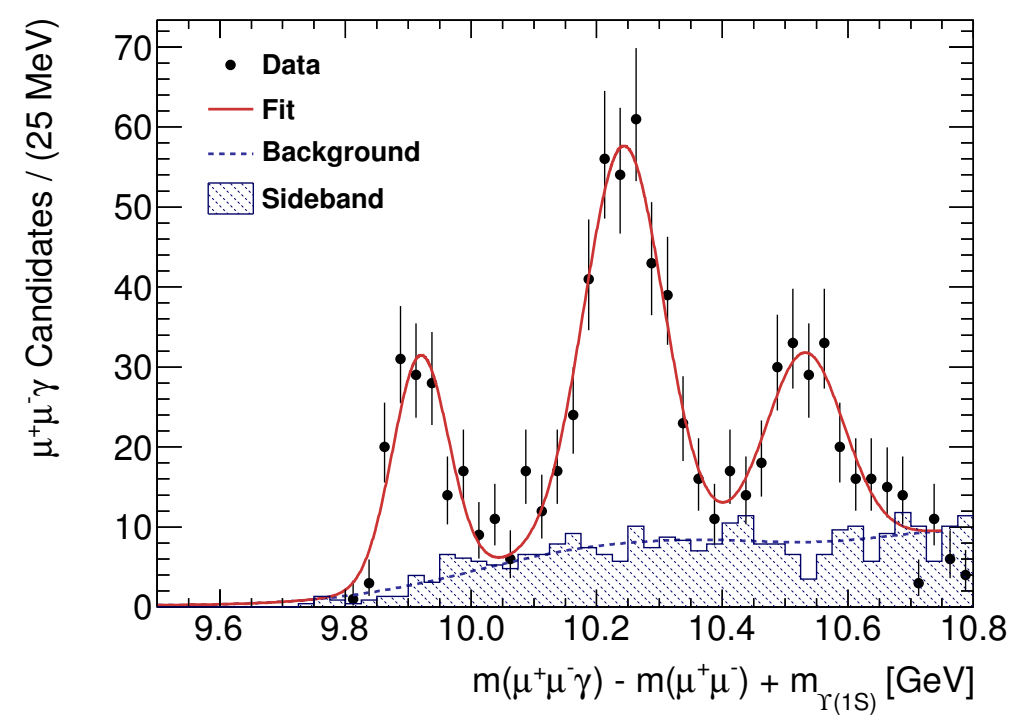

Figure 6.8: An alternative fit to the $m\left(\mu^{+} \mu^{-} \gamma\right)-m\left(\mu^{+} \mu^{-}\right)+m_{\Upsilon(1 S)}$ distribution for $\chi_{b}(n P) \rightarrow \Upsilon(1 S) \gamma$ candidates. The alternative background PDF is derived by performing the Gaussian kernel estimation procedure on the "low mass" sideband distribution [123], as discussed in Section 6.6.

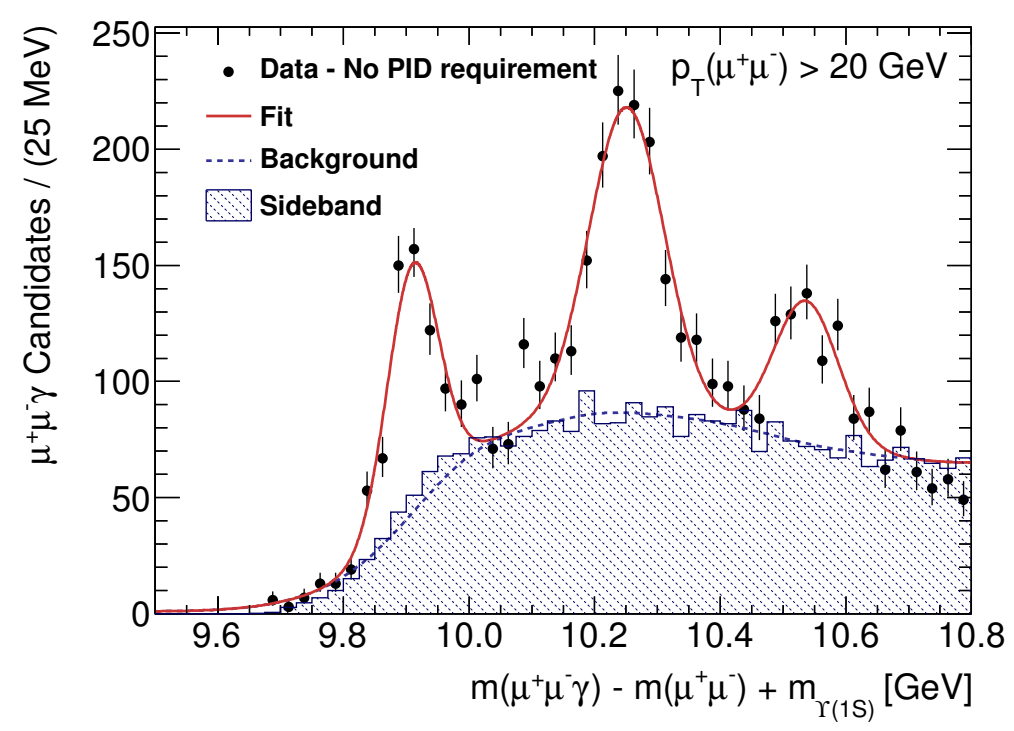

Figure 6.9: Fit to $m\left(\mu^{+} \mu^{-} \gamma\right)-m\left(\mu^{+} \mu^{-}\right)+m_{\Upsilon(1 S)}$ distribution for $\chi_{b}(n P) \rightarrow \Upsilon(1 S) \gamma$ candidates with no "loose" photon identification requirement imposed. The fit procedure is identical to the nominal fit but an alternative background PDF is used, derived by performing the Gaussian kernel estimation procedure on the "low mass" sideband distribution [123]. 

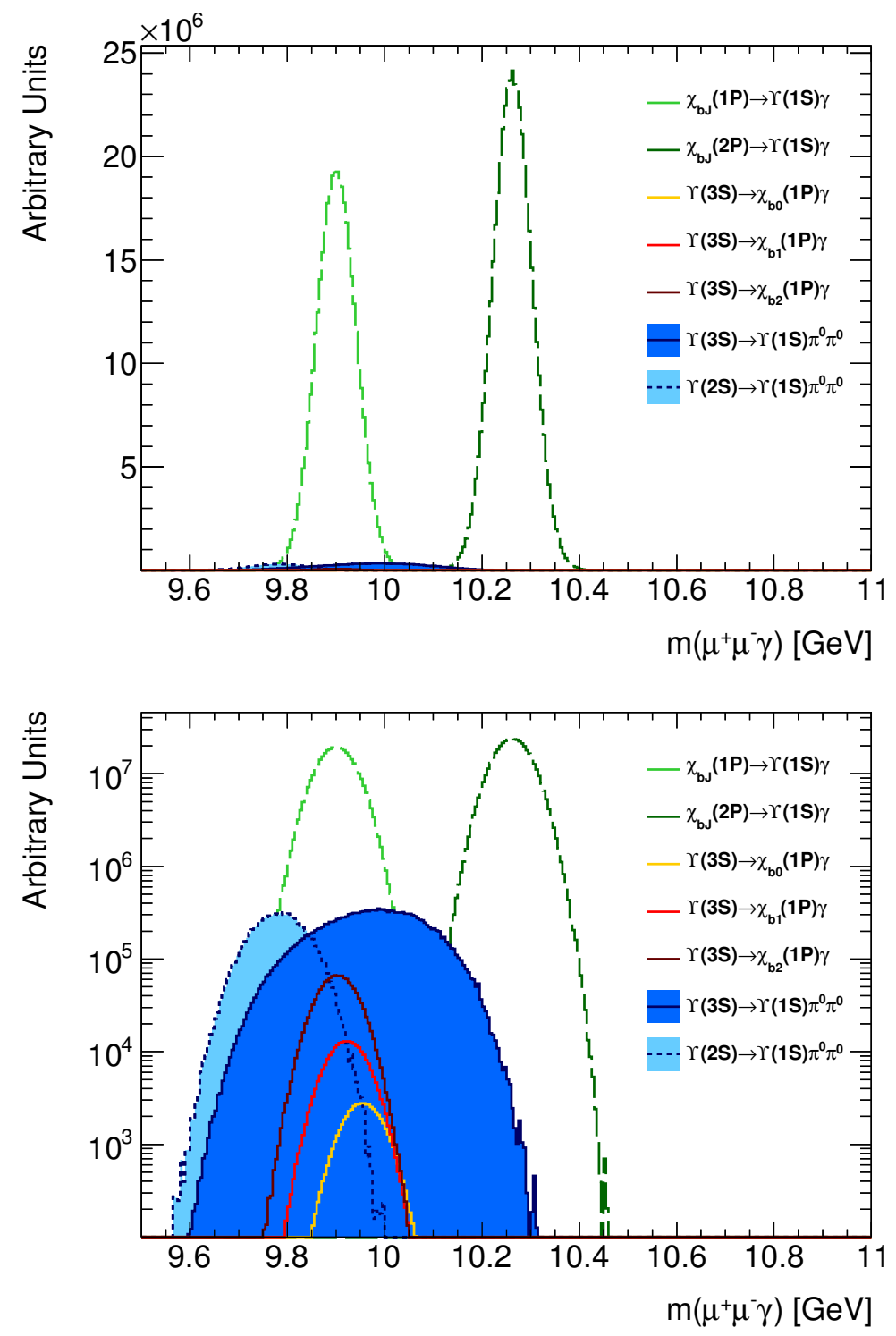

Figure 6.10: Simulation of the shape of contributions to the mass difference distribution from partially reconstructed bottomonium decays resulting in a $\Upsilon(1 S) \gamma$ final state. The simulation uses the $\Upsilon(m S)$ differential cross sections measured by ATLAS as an input [87]. The simulation approximates the inclusive $\chi_{b J}(1 P)$ and $\chi_{b J}(2 P)$ cross sections (i.e. the sum of all three spin states) to the measured $\Upsilon(1 S)$ and $\Upsilon(2 S)$ cross sections respectively, scaled by 0.75 (this approximation and scaling is motivated by the prompt $\chi_{c J}$ cross section relative to the prompt $J / \psi$ cross section, measured in Chapter 7). The simulation applies all fiducial cuts on final state particles and smears $m\left(\mu^{+} \mu^{-} \gamma\right)$ by $\pm 40 \mathrm{MeV}$ to mimic the experimental mass resolution (modelling of reconstruction efficiencies is neglected). All channels are normalised with the appropriate world average branching fractions [3]. The decay chains considered all produce smooth and broad contributions to the distribution and together result in contributions (relative to the $\chi_{b}(1 P)$ peak) to the mass difference distribution of less than $5 \%$. 


\subsection{Results}

The measured experimental mass barycentres for the $\chi_{b}(1 P), \chi_{b}(2 P)$ and $\chi_{b}(3 P)$ candidate signals are shown in Table 6.3. The measured $\chi_{b}(1 P)$ and $\chi_{b}(2 P)$ experimental mass barycentres cannot be directly compared to the world average values for the $\chi_{b J}(1,2 P)$ masses (since they represent an unknown mixture of the $J=0,1,2$ states) but some assumptions on the relative production rates can be made in order to compare with existing measurements. To a good approximation, the contribution from the $J=0$ states can be neglected, owing to their lower radiative branching fractions relative to the $J=1,2$ states. The production rate of the $\chi_{b 2}(1 P)$ state relative to the $\chi_{b 1}(1 P)$ state has been measured by the CMS experiment in $p p$ collisons at $\sqrt{s}=8 \mathrm{TeV}$ to be around 0.5 in radiative decays to $\Upsilon(1 S)$, with no strong dependence on $\Upsilon(1 S)$ transverse momentum [125]. The relative production rate measured by CMS represents the product $\mathcal{B}\left(\chi_{b 2}(1 P) \rightarrow \Upsilon(1 S) \gamma\right) \cdot \sigma\left(\chi_{b 2}(1 P)\right) / \mathcal{B}\left(\chi_{b 1}(1 P) \rightarrow \Upsilon(1 S) \gamma\right) \cdot \sigma\left(\chi_{b 1}(1 P)\right)$ and can be

used to compute a production weighted average of the world average $\chi_{b 1}(1 P)$ and $\chi_{b 2}(1 P)$ masses that can be compared to the measured $\chi_{b}(1 P)$ experimental mass barycentre. No data exist on the total production rate of the $\chi_{b 2}(2 P)$ state relative to the $\chi_{b 1}(2 P)$ state, so the measured value from the $\chi_{b}(1 P)$ system is adopted as an approximation. The weighted average is determined from

$$
\bar{m}_{n}=\frac{m_{1}(n P)+R \cdot m_{2}(n P)}{1+R}
$$

where $m_{J}(n P)$ is the world average value for the mass of the $\chi_{b J}(n P)$ state and $R$ is the measured production rate of the $\chi_{b 2}(1 P)$ state relative to the $\chi_{b 1}(1 P)$ state. This equation gives production-weighted averages of $9899.4 \pm 0.9 \mathrm{MeV}$ and $10260.0 \pm 0.6 \mathrm{MeV}$ for the $\chi_{b}(1 P)$ and $\chi_{b}(2 P)$ triplets respectively. These calculated production-weighted averages are in agreement with the measured experimental mass barycentres shown in Table 6.3. The measured experimental mass barycen- 
tre for the $\chi_{b}(3 P)$ candidate is in agreement with the theoretical predictions for the spin averaged mass centre of gravity of the $\chi_{b}(3 P)$ triplet (shown in Table 6.2) within the experimental uncertainty, though the central value is around $15 \mathrm{MeV}$ higher than both predictions.

\begin{tabular}{|l|l|}
\hline States & Experimental Mass Barycentre \\
\hline$\chi_{b}(1 P)$ & $9910 \pm 6$ (stat.) \pm 11 (syst.) $\mathrm{MeV}$ \\
$\chi_{b}(2 P)$ & $10246 \pm 5$ (stat.) \pm 18 (syst.) $\mathrm{MeV}$ \\
$\chi_{b}(3 P)$ & $10541 \pm 11$ (stat.) \pm 30 (syst.) $\mathrm{MeV}$ \\
\hline
\end{tabular}

Table 6.3: The measured experimental mass barycentres for the observed $\chi_{b}$ signals.

The statistical significance of the $\chi_{b}(3 P)$ candidate signal is quantified with the likelihood ratio test. The statistical significance $Z$ is given by,

$$
Z=\sqrt{-2 \ln \left(\frac{L_{\mathrm{s}+\mathrm{b}}}{L_{\mathrm{b}}}\right)}
$$

where $L_{\mathrm{s}+\mathrm{b}}$ is the maximum likelihood value for the fit performed with the nominal model containing signal and $L_{\mathrm{b}}$ is the maximum likelihood value for the fit performed with no signal component for the $\chi_{b}(3 P)$ candidate peak (the background-only hypothesis) [126]. The background only fit was achieved by implementing a parameter constraint of $f_{3}=0$ (see Equation 6.1). Using this method, the statistical significance of the $\chi_{b}(3 P)$ candidate signal is estimated to be in excess of 6 standard deviations. The calculation of the statistical significance is repeated for each set of systematic variations in the fit model discussed in Section 6.6 and is found to remain in excess of 6 standard deviations in each case. 


\subsection{Converted Photon Analysis}

An additional, similar analysis using photon conversions $\left(\gamma \rightarrow e^{+} e^{-}\right)$measured in the inner detector to reconstruct $\chi_{b}(n P) \rightarrow \Upsilon(m S) \gamma$ decays was performed in parallel with the unconverted photon analysis. This analysis is described in detail in Ref. [66] and the results will be briefly reviewed here, to complement the detailed discussion of the unconverted photon analysis.

The use of photon conversions offers improved mass resolution and access to lower energy photons than unconverted photons reconstructed with the EM calorimeter. However, the overall efficiency to reconstruct photon conversions is considerably lower than for unconverted photons, for a given photon transverse momentum (for $\left.p_{T}^{\gamma}>2.5 \mathrm{GeV}\right)$. The converted photon analysis used an identical dataset and the same selection of $\Upsilon(m S) \rightarrow \mu^{+} \mu^{-}$candidates as described in Section 6.2.2. Although the same dataset was used, the photons reconstructed from $e^{+} e^{-}$conversions in the inner detector represent a statistically independent sample from the unconverted photons reconstructed with the EM calorimeter. Further to this (for the dataset used) no events were found where an $\Upsilon(m S) \gamma$ candidate was selected by both analyses.

\subsubsection{Photon Conversion Selection}

Photon conversions are reconstructed from pairs of oppositely charged tracks with transverse momentum $p_{T}>500 \mathrm{MeV}$, whose opening angle and hence invariant mass are both consistent with zero. The reconstruction of photon conversions in ATLAS is described in more detail in Section 5.3.1. Candidate photon conversions selected by the ATLAS conversion finding algorithm are required to satisfy the following additional requirements:

- The radial displacement, $R$, of the conversion vertex from the beam axis must satisfy $R>40 \mathrm{~mm}$, to reject backgrounds from promptly produced tracks 
- Each candidate electron/positron track is required to have been reconstructed from at least 4 hits in the silicon tracking detectors (Pixel and SCT)

- The $\chi^{2}$ probability of the vertex fit is required to be greater than 0.01

- Candidate photon conversions are required to have transverse momentum $p_{T}^{\gamma}>$ $1.0 \mathrm{GeV}$ and pseudo-rapidity $\left|\eta^{\gamma}\right|<2.3$

- Any photon conversion candidates reconstructed from tracks already identified as muons are rejected

Candidate photon conversions passing these requirements are associated with $\Upsilon(m S) \rightarrow \mu^{+} \mu^{-}$candidates, selected as described in Section 6.2.2, to form $\chi_{b}(n P) \rightarrow$ $\Upsilon(m S) \gamma$ candidates. The transverse momentum of the di-muon system is required to satisfy $p_{T}^{\mu^{+} \mu^{-}}>12 \mathrm{GeV}$. In addition to these requirements, the 3-dimensional impact parameter, $a_{0}$, between the conversion momentum (passing through the conversion vertex) and the di-muon vertex is required to satisfy $a_{0}<2 \mathrm{~mm}$, to reject $\mu^{+} \mu^{-} \gamma$ combinations incompatible with having originated from the same decay vertex. The $a_{0}$ parameter is defined and described in Section 5.6.

\subsubsection{Observation of $\chi_{b}(n P) \rightarrow \Upsilon(m S) \gamma$ with photon conver- sions}

The mass difference distributions $m\left(\mu^{+} \mu^{-} \gamma\right)-m\left(\mu^{+} \mu^{-}\right)+m_{\Upsilon(1 S)}$ and $m\left(\mu^{+} \mu^{-} \gamma\right)-$ $m\left(\mu^{+} \mu^{-}\right)+m_{\Upsilon(2 S)}$, for $\mu^{+} \mu^{-} \gamma$ candidates reconstructed with a di-muon invariant mass within the $\Upsilon(1 S)$ and $\Upsilon(2 S)$ mass windows (shown in Figure 6.1) respectively are shown in Figure 6.11. The $m\left(\mu^{+} \mu^{-} \gamma\right)-m\left(\mu^{+} \mu^{-}\right)+m_{\Upsilon(1 S)}$ distribution exhibits mass peaks compatible with the $\chi_{b J}(1 P)$ and $\chi_{b J}(2 P)$ states at around $9.9 \mathrm{GeV}$ and $10.2 \mathrm{GeV}$ respectively. An additional structure at a mass of around $10.5 \mathrm{GeV}$ is also observed in both the $m\left(\mu^{+} \mu^{-} \gamma\right)-m\left(\mu^{+} \mu^{-}\right)+m_{\Upsilon(1 S)}$ and $m\left(\mu^{+} \mu^{-} \gamma\right)-m\left(\mu^{+} \mu^{-}\right)+m_{\Upsilon(2 S)}$ distributions. The mass of these two structures 
are compatible with the measured experimental mass barycentre of the $\chi_{b}(3 P)$ candidate signal observed with unconverted photons shown in Figure 6.7. These two structures are interpreted as the decays of the same $\chi_{b}(3 P)$ candidate signal observed with unconverted photons, into $\Upsilon(1 S) \gamma$ and $\Upsilon(2 S) \gamma$ final states, respectively.

The lower minimum photon transverse momentum threshold $\left(p_{T}^{\gamma}>1.0 \mathrm{GeV}\right)$ of photons reconstructed from conversions offers a kinematic acceptance for $\chi_{b}(n P) \rightarrow$ $\Upsilon(m S) \gamma$ decays that is much greater than for unconverted photons reconstructed with the EM calorimeter $\left(p_{T}^{\gamma}>2.5 \mathrm{GeV}\right)$, for $\chi_{b}$ candidates with a given transverse momentum. Figure 6.12 shows the kinematic acceptance for $\chi_{b}(n P) \rightarrow \Upsilon(m S) \gamma$ decays as a function of $\chi_{b}$ transverse momentum, for photons with minimum transverse momentum thresholds corresponding to those imposed for converted photons and for unconverted photons. The photon energy in the $\chi_{b}$ rest frame of a $\chi_{b}(n P) \rightarrow \Upsilon(m S) \gamma$ decay varies significantly for the different allowed combinations of $n$ and $m$, leading to large variations in acceptance between the decay channels. The observation of a significant $\chi_{b}(3 P) \rightarrow \Upsilon(2 S) \gamma$ signal with photon conversions and not with unconverted photons, for the same data sample and $\Upsilon(2 S) \rightarrow \mu^{+} \mu^{-}$ selection, is in agreement with these expectations, based on considerations of kinematic acceptance alone.

A simultaneous unbinned maximum likelihood fit is made to the $m\left(\mu^{+} \mu^{-} \gamma\right)-$ $m\left(\mu^{+} \mu^{-}\right)+m_{\Upsilon(1 S)}$ and $m\left(\mu^{+} \mu^{-} \gamma\right)-m\left(\mu^{+} \mu^{-}\right)+m_{\Upsilon(2 S)}$ distributions. This exploits both observed decay modes to measure the experimental mass barycentre of the $\chi_{b}(3 P)$ candidate and the known $\chi_{b}(2 P)$ and $\chi_{b}(1 P)$ masses are used to constrain the mass scale. The experimental mass barycentre of the $\chi_{b}(3 P)$ candidate measured with converted photons is found to be $10.530 \pm 0.005$ (stat.) \pm 0.009 (syst.) $\mathrm{GeV}$, in agreement with the measurement performed with unconverted photons. The observation of two decay modes, in addition to the improved mass resolution, endows the converted photon analysis with much more sensitivity and provides a measurement of the experimental mass barycentre of the $\chi_{b}(3 P)$ candidate that is significantly 


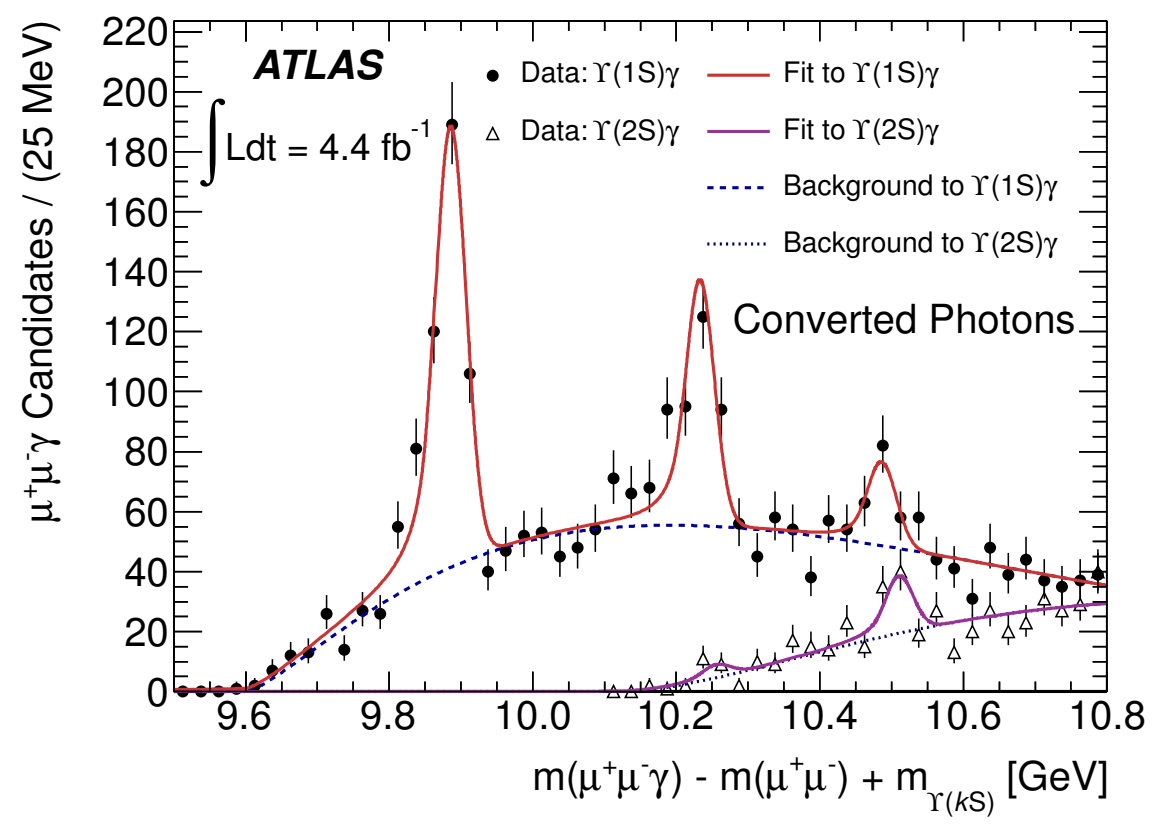

Figure 6.11: The result of a simultaneous unbinned maximum likelihood fit to the $m\left(\mu^{+} \mu^{-} \gamma\right)-m\left(\mu^{+} \mu^{-}\right)+m_{\Upsilon(1 S)}$ and $m\left(\mu^{+} \mu^{-} \gamma\right)-m\left(\mu^{+} \mu^{-}\right)+m_{\Upsilon(2 S)}$ distributions for $\chi_{b}(n P) \rightarrow \Upsilon(k S) \gamma$ candidates reconstructed with converted photons.

more precise than that obtained with the unconverted photons. The statistical significance of the $\chi_{b}(3 P)$ signal observed with photon conversions (combining both $\Upsilon(1,2 S) \gamma$ decay modes $)$ is in excess of 6 standard deviations. 

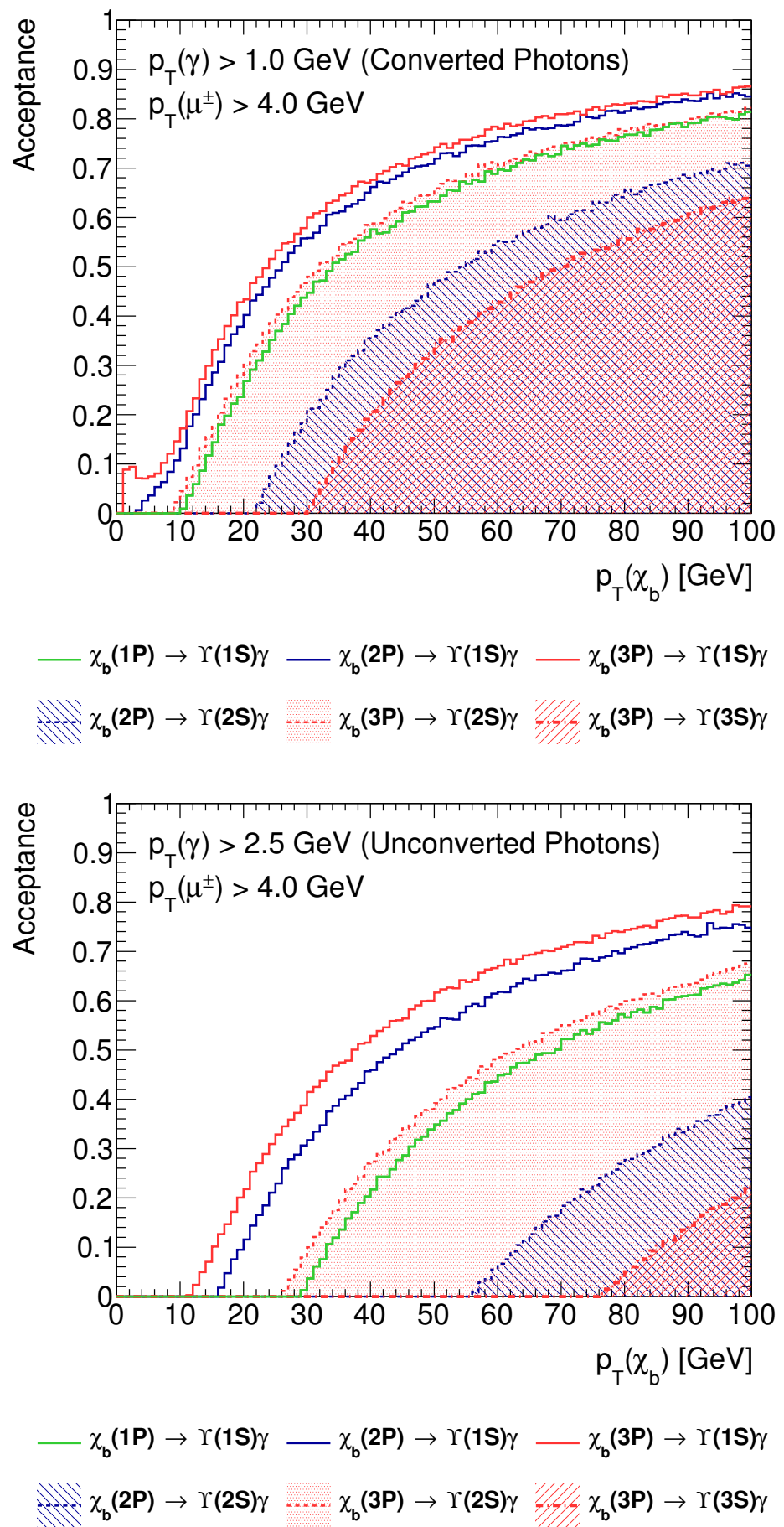

Figure 6.12: The kinematic acceptance of $\chi_{b}(n P) \rightarrow \Upsilon(m S) \gamma$ decays reconstructed with converted (upper) and unconverted (lower) photons. The kinematic acceptance for $\chi_{b}(n P) \rightarrow \Upsilon(m S) \gamma$ decays reconstructed with converted photons is significantly greater than for unconverted photons, for a given $\chi_{b}$ transverse momentum. 


\subsection{Confirmation by Other Experiments}

Following the ATLAS observation of the $\chi_{b}(3 P)$ candidate, both the D0 and LHCb experiments confirmed the observation of a new state decaying to $\Upsilon(1 S) \gamma[127,128]$.

The D0 experiment used a data sample corresponding to $1.3 \mathrm{fb}^{-1}$ of $p \bar{p}$ collision data collected at $\sqrt{s}=1.96 \mathrm{TeV}$ to search for evidence of new bottomonium states decaying to $\Upsilon(1 S) \gamma$. $\Upsilon(1 S)$ candidates were reconstructed from the decay $\Upsilon(1 S) \rightarrow \mu^{+} \mu^{-}$and photons were reconstructed from $\gamma \rightarrow e^{+} e^{-}$conversions in the inner layers of the tracking detector. The di-muon invariant mass distribution of reconstructed $\Upsilon(n S) \rightarrow \mu^{+} \mu^{-}$candidates is shown Figure 6.13; the experimental resolution in $m\left(\mu^{+} \mu^{-}\right)$is similar to that achieved by ATLAS. The mass difference distribution $M_{\mu^{+} \mu^{-} \gamma}-M_{\mu^{+} \mu^{-}}+M_{\Upsilon(1 S)}$ of $\chi_{b} \rightarrow \Upsilon(1 S) \gamma$ candidates reconstructed by D0 is shown in Figure 6.13. Mass peaks corresponding to the $\chi_{b}(1 P)$ and $\chi_{b}(2 P)$ states are visible at masses around $9.9 \mathrm{GeV}$ and $10.2 \mathrm{GeV}$ respectively. The D0 experiment also observed an additional mass peak at around $10.5 \mathrm{GeV}$, with a width consistent with experimental resolution, qualitatively similar to the structure observed by ATLAS. The statistical significance of the third mass peak was determined to be in excess of six standard deviations using the same method described in Section 6.7. The mass of the new structure was measured to be $10.551 \pm 0.014$ (stat.) \pm 0.017 (syst.) GeV, in agreement with both (calorimetry and conversions) ATLAS measurements. D0 suggest that the mass peak could be attributable to an exotic bottomonium state (analogous to the $X(3872)$ state in the charmonium sector) rather than the $\chi_{b}(3 P)$ triplet but note that further analysis would be necessary to confirm this hypothesis [127].

Following the D0 confirmation of the new state, LHCb presented a preliminary $\chi_{b}$ analysis based upon a data sample of $p p$ collision data collected at $\sqrt{s}=7 \mathrm{TeV}$, corresponding to $0.9 \mathrm{fb}^{-1}$ of integrated luminosity. The $\chi_{b}$ states were reconstructed in the $\Upsilon(1 S) \gamma$ channel with $\Upsilon(1 S) \rightarrow \mu^{+} \mu^{-}$. Photons were reconstructed with 
the $\mathrm{LHCb}$ electromagnetic calorimeter. The di-muon invariant mass distribution of $\Upsilon(n S) \rightarrow \mu^{+} \mu^{-}$candidates reconstructed by LHCb is shown Figure 6.14. The di-muon mass resolution of the LHCb detector is superior to that achieved by both ATLAS and D0 in the $\Upsilon(n S)$ region, owing to its specialised tracking detectors, optimised for the reconstruction of heavy flavour hadron decays. The mass difference distribution $m\left(\mu^{+} \mu^{-} \gamma\right)-m\left(\mu^{+} \mu^{-}\right)$of $\chi_{b} \rightarrow \Upsilon(1 S) \gamma$ candidates reconstructed by LHCb is shown in Figure 6.14. Mass peaks corresponding to the $\chi_{b}(1 P)$ and $\chi_{b}(2 P)$ states are visible at masses differences around $0.5 \mathrm{GeV}$ and $0.8 \mathrm{GeV}$ respectively. An additional mass peak qualitatively consistent with that observed by ATLAS and D0 was observed at around $1.1 \mathrm{GeV}$ in the mass difference distribution. The statistical significance of the third mass peak was assessed to be excess of 12 standard deviations and was also calculated using the method described in Section 6.7. LHCb interpret the third peak as evidence for the $\chi_{b}(3 P)$ triplet and measure an experimental mass barycentre of $10535 \pm 10$ (stat.) $\mathrm{MeV}$ and only quantify the statistical uncertainty associated with the measurement. The mass measurement of $\mathrm{LHCb}$ is consistent with both ATLAS measurements and the D0 measurement. 

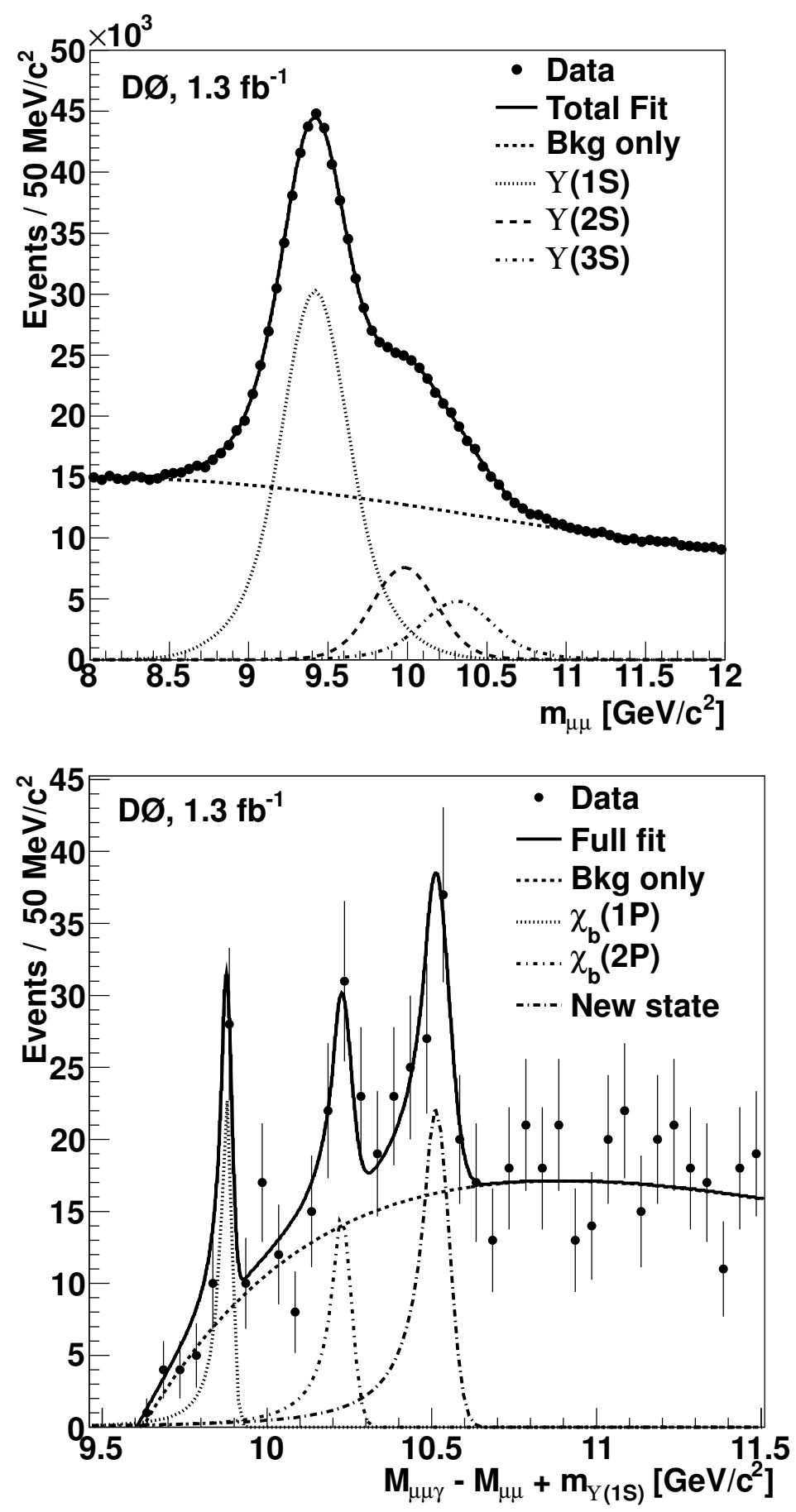

Figure 6.13: The di-muon invariant mass distribution of $\Upsilon(n S) \rightarrow \mu^{+} \mu^{-}$candidates reconstructed by the D0 experiment [127] (upper). The mass difference distribution $M_{\mu^{+} \mu^{-} \gamma}-M_{\mu^{+} \mu^{-}}+M_{\Upsilon(1 S)}$ of $\chi_{b} \rightarrow \Upsilon(1 S) \gamma$ candidates reconstructed by D0 [127] (lower). Mass peaks corresponding to the $\chi_{b}(1 P)$ and $\chi_{b}(2 P)$ states, along with the new higher mass state, compatible with the $\chi_{b}(3 P)$ candidate observed by ATLAS, are visible. 

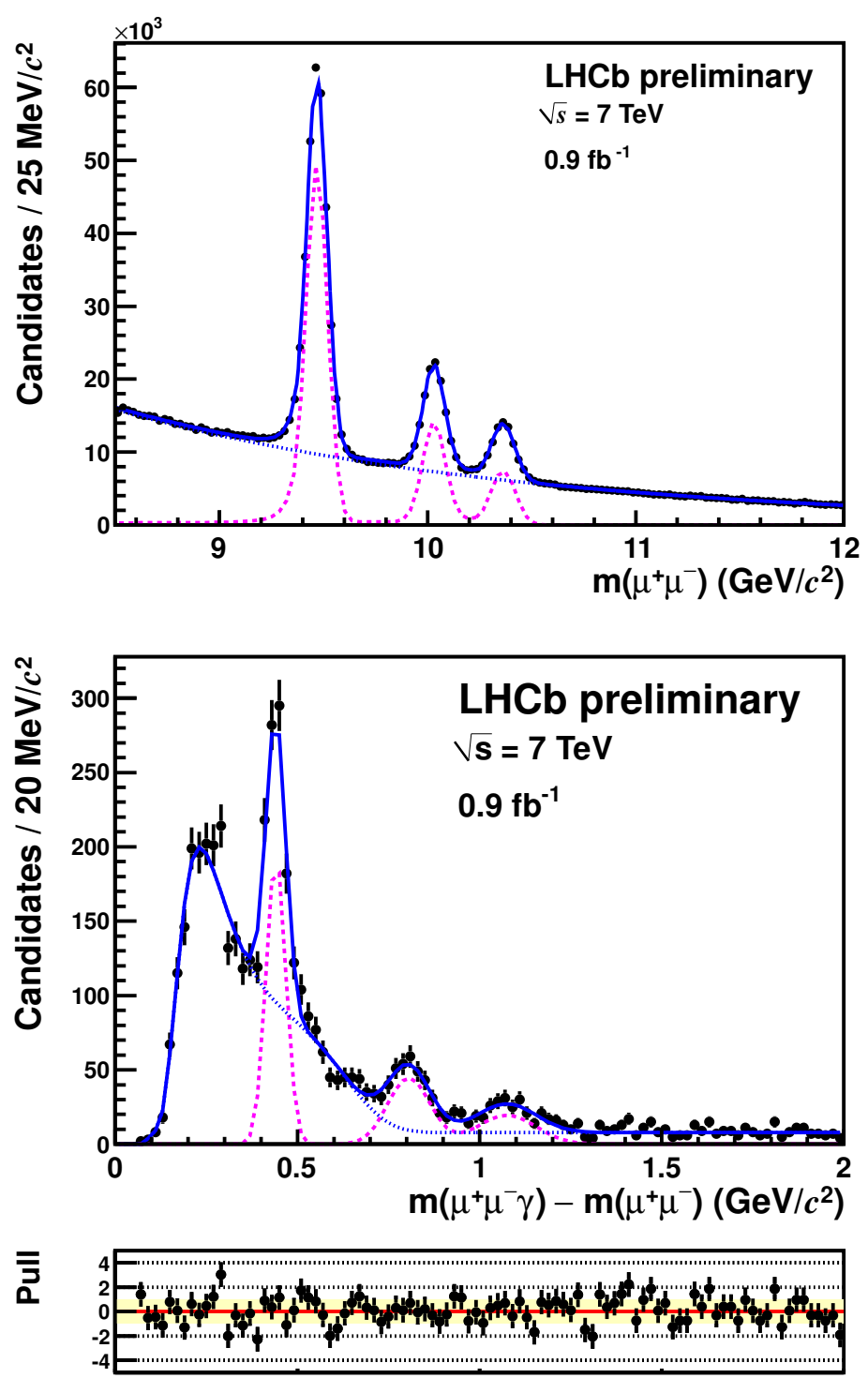

Figure 6.14: The di-muon invariant mass distribution of $\Upsilon(n S) \rightarrow \mu^{+} \mu^{-}$candidates reconstructed by the LHCb experiment [128] (upper). The mass difference distribution $m\left(\mu^{+} \mu^{-} \gamma\right)-m\left(\mu^{+} \mu^{-}\right)$of $\chi_{b} \rightarrow \Upsilon(1 S) \gamma$ candidates reconstructed by LHCb [128] (lower). Mass peaks corresponding to the $\chi_{b}(1 P)$ and $\chi_{b}(2 P)$ states, along with the $\chi_{b}(3 P)$ candidate, compatible with that observed by ATLAS, are visible. 


\subsection{Discussion}

The mass of the new state has been measured by several different experiments yet this is one of only two known physical properties of the state. The fact that the state decays to $\Upsilon(n S) \gamma$ requires that the state have positive charge conjugation parity, though no other quantum numbers have been determined. The interpretation of the new state $(\mathrm{s})$ as the $\chi_{b}(3 P)$ triplet is well motivated from theoretical predictions and from the qualitative behaviour of the observed (uncorrected) production rates in decays to both $\Upsilon(1 S) \gamma$ and $\Upsilon(2 S) \gamma$. The conventional quarkonium model has also been fully verified experimentally, with the masses of many recently discovered "missing" states agreeing well with a priori theoretical predictions $[11,129]$. However, several other possible interpretations for the new state(s) exist. Exotic bottomonium-like states, $X_{b}$, analogous to the $X(3872)$ in the charmonium sector, have been predicted in various tetraquark and meson molecule models [130-132]. The predicted states have masses close to the $B \bar{B}$ threshold and are $C$ even, allowing decays to $\Upsilon(n S) \gamma$, though $B \bar{B}$ and $\Upsilon(n S) \pi \pi$ decays are expected to dominate. Such states have been searched for at the LHC in $\Upsilon(1 S) \pi^{+} \pi^{-}$final states and are excluded at $95 \%$ confidence level for masses between 10 to $11 \mathrm{GeV}$ and for production cross sections above $6 \%$ of the prompt $\Upsilon(2 S)$ cross section [133]. If the expectation that $\mathcal{B}\left(X_{b} \rightarrow \Upsilon(1 S) \pi^{+} \pi^{-}\right)>\mathcal{B}\left(X_{b} \rightarrow \Upsilon(1 S) \gamma\right)$ is correct for such hypothetical states (as is the case for the analogous radiative and pionic decays of the $X(3872)$ ), then the observation of $X_{b} \rightarrow \Upsilon(1 S) \gamma$ is difficult to reconcile with a strong experimental exclusion of $X_{b} \rightarrow \Upsilon(1 S) \pi^{+} \pi^{-}$for the same state.

There is much scope for further analysis to shed more light on the nature of the new state. The mass splitting between the $J=1$ and $J=2$ states of the $\chi_{b J}(3 P)$ triplet is expected to be around $12 \mathrm{MeV}$ (see Tables 6.2 and 6.4). Improvements in the experimental mass resolution could allow fine structure to be resolved if present, which could add more weight to the $\chi_{b}(3 P)$ interpretation but would not 
unambiguously confirm it. The mass difference resolution achieved by the CMS experiment in recent measurements of the $\chi_{b}(1 P)$ triplet is sufficient to discern the $\chi_{b 1}(1 P)$ and $\chi_{b 2}(1 P)$ states as individual mass peaks, suggesting that the CMS detector might have some sensitivity to possible fine structure within the mass peak of the new state [125]. An analysis of the angular distributions of the final state particles in $\Upsilon(n S) \gamma \rightarrow \mu^{+} \mu^{-} \gamma$ decays could allow the spin and parity quantum numbers of the new state to be determined. Such an analysis would ideally have the ability to resolve any fine structure, since an unresolved composite $\chi_{b J}(3 P)$ peak would contain an unknown mixture of $0^{++}, 1^{++}$and $2^{++}$components.

The existence of a previously unknown bottomonium state with a mass below the $B \bar{B}$ threshold has several important implications for the current understanding of inclusive bottomonium production at hadron colliders. While the total production rate of the $\chi_{b}(3 P)$ candidate has not been measured, the observed number of events relative to the $\chi_{b}(1 P)$ and $\chi_{b}(2 P)$ states (and the known acceptance shown in Figure 6.12) suggests that the production rate and radiative branching fractions of the new state are comparable to that of the $\chi_{b}(1 P)$ and $\chi_{b}(2 P)$ states. The radiative decays of the $\chi_{b}(3 P)$ candidate represent a source of feed-down to the inclusive $\Upsilon(n S)$ production rates that was previously not considered in theoretical calculations. If the new state(s) is indeed the $\chi_{b}(3 P)$ states, then the branching fraction for the decay $\chi_{b J}(3 P) \rightarrow \Upsilon(3 S) \gamma$ is expected to be large (in analogy with the corresponding decays of the $\chi_{b}(1 P)$ and $\chi_{b}(2 P)$ states) [115]. This would represent a significant source of feed-down to the total $\Upsilon(3 S)$ production rate that was previously thought to result from direct production alone (as is the case for prompt $\psi(2 S)$ production in the charmonium system). This new knowledge confutes the belief that $\Upsilon(3 S)$ production could provide a clean window on the direct bottomonium production mechanism.

The discovery of the $\chi_{b}(3 P)$ candidate stimulated renewed interest in theoretical predictions of the spectroscopy of the bottomonium system. Several new potential 
model calculations of the $\chi_{b J}(3 P)$ masses were published following the discovery of the experimental candidate for the $\chi_{b}(3 P)$ states [134-139]. These recent calculations are summarised in Table 6.4. In addition to purely theoretical predictions, Ref. [135] used the candidate $\chi_{b}(3 P)$ experimental mass barycentre measured by ATLAS (conversion result), in combination with a QCD-derived potential model prediction for the $3 P$ fine structure splittings to derive data driven estimates for the $\chi_{b J}(3 P)$ masses of $10502 \pm 10 \mathrm{MeV}, 10524 \pm 10 \mathrm{MeV}$ and $10539 \pm 10 \mathrm{MeV}$ for the $J=0,1,2$ states, respectively. In general, the post discovery calculations are in broad agreement with the early predictions shown in Table 6.2, though the more recent calculations tend to predict higher masses for the $\chi_{b J}(3 P)$ states with c.o.g. masses closer to the measured experimental mass barycentre. This could be due to advancements in theoretical techniques, new constraints on free parameters from recent data or (perhaps cynically) a manifestation of "theoretician's bias".

\begin{tabular}{|l|c|c|c|c|}
\hline \multirow{2}{*}{ Author(s) } & \multicolumn{4}{|c|}{ Predicted $\chi_{b J}(3 P)$ mass [MeV] } \\
\cline { 2 - 5 } & $J=0$ & $J=1$ & $J=2$ & c.o.g. \\
\hline Radford \& Repko (Ref. [134]) & 10516.0 & 10538.1 & 10552.9 & 10544.4 \\
Dib \& Neill (Ref. [135]) & 10515.0 & 10538.1 & 10552.9 & 10543.9 \\
Radford \& Repko (Ref. [136]) & 10527.2 & 10544.3 & 10555.6 & 10548.7 \\
Ferretti \& Santopinto (Ref. [138]) & 10494 & 10511 & 10524 & 10516.3 \\
Wei-Zhao et al. (Ref. [139]) & 10491.3 & 10527.6 & 10541.4 & 10531.2 \\
\hline
\end{tabular}

Table 6.4: Theoretical predictions for the masses of the $\chi_{b J}(3 P)$ mass states published after the discovery of the experimental candidate for the $\chi_{b}(3 P)$ states. The centre of gravity (c.o.g.) of the triplet is the spin averaged mass of the three individual states.

\subsection{Conclusion}

The $\chi_{b}$ bottomonium states are observed through the reconstruction of the radiative decay $\chi_{b}(n P) \rightarrow \Upsilon(1 S) \gamma$ using $4.4 \mathrm{fb}^{-1}$ of $p p$ collision data collected at $\sqrt{s}=7 \mathrm{TeV}$ with the ATLAS experiment. Peaks in the mass difference distribution $m\left(\mu^{+} \mu^{-} \gamma\right)-m\left(\mu^{+} \mu^{-}\right)+m_{\Upsilon(1 S)}$ of reconstructed $\Upsilon(1 S) \gamma$ candidates corresponding 
to the $\chi_{b}(1 P)$ and $\chi_{b}(2 P)$ triplets are observed at masses consistent with the world average values. An additional mass peak, consistent with theoretical expectations for the $\chi_{b}(3 P)$ triplet is also observed. The experimental mass barycentre of the $\chi_{b}(3 P)$ candidate is measured (using photons reconstructed in the EM calorimeter) to be $10541 \pm 11$ (stat.) \pm 30 (syst.) $\mathrm{MeV}$. The observation of a new state decaying to $\Upsilon(1 S) \gamma$, consistent with theoretical expectations for the $\chi_{b}(3 P)$ triplet, is confirmed by an independent ATLAS analysis using photon conversions and subsequent measurements from the D0 and LHCb experiments [66, 127, 128]. 


\section{Chapter 7}

\section{MEASUREMENT OF THE PRODUCTION OF THE $\chi_{c 1}$ AND $\chi_{c 2}$ CHARMONIUM STATES}

The following analysis of $\chi_{c 1}$ and $\chi_{c 2}$ production represents a more developed version of the preliminary analysis presented in [140]. Following the initial submission of this thesis, the analysis described in this Chapter was prepared for publication. The preprint can be found in Ref. [141].

\subsection{Introduction to the $\chi_{c}$ system}

The $\chi_{c}$ states are the spin-triplet $(S=1) P$-wave $(L=1)$ states of the charmonium system, analogous to the $\chi_{b}$ states in the bottomonium system discussed in Chapter 6 . While the bottomonium system contains at least two triplets of $\chi_{b}$ states below the open-flavour threshold (pending the confirmation of the nature the $\chi_{b}(3 P)$ candidate discussed in Chapter 6), only a single triplet of $\chi_{c}$ states is known to exist below the open-charm threshold. As in the $\chi_{b}$ system, the three states of each $\chi_{c J}$ triplet have total angular momentum, parity and charge conjugation parity quantum numbers $J^{P C}=0^{++}, 1^{++}, 2^{++}$and are denoted $\chi_{c J}(n P)$, where $n$ is the radial quantum number. Since only a single triplet exists below the open-charm threshold, the $\chi_{c J}(1 P)$ states are often simply denoted $\chi_{c J}$, a notation which will be adopted in the following discussion (when the distinction is unambiguous). However, experimental candidates do exist for the $\chi_{c J}(2 P)$ states, with masses close to the open-charm 
threshold. The $X(3915)$ state observed by Belle in decays to $J / \psi \omega[142]$, and confirmed by BaBar [143], has been identified as a candidate for the $\chi_{c 0}(2 P)$ state [3]. Belle also identified a candidate for the $\chi_{c 2}(2 P)$ state in decays to $D \bar{D}$ [144], which was subsequently confirmed by BaBar [145]. It has been argued that the $X(3872)$, now confirmed to be a $1^{++}$state [26], could be the $\chi_{c 1}(2 P)$ state, but with a mass and decay width distorted from the charmonium expectation through a strong coupling to intermediate $D \bar{D}$ states $[146,147]$. However, the properties of these candidate $2 P$ states are not well known and it is not clear that they are consistent with being "conventional" charmonium states (the $X(3872)$ in particular).

\begin{tabular}{|c|c|c|c|c|}
\hline$J$ & Mass $m\left(\chi_{c J}\right)$ & $\Delta m$ & Width & $\mathcal{B}\left(\chi_{c J}(1 P) \rightarrow J / \psi \gamma\right)$ \\
\hline 0 & $3414.75 \pm 0.31 \mathrm{MeV}$ & $317.83 \mathrm{MeV}$ & $10.3 \pm 0.6 \mathrm{MeV}$ & $(1.30 \pm 0.07) \%$ \\
1 & $3510.66 \pm 0.07 \mathrm{MeV}$ & $413.74 \mathrm{MeV}$ & $0.86 \pm 0.05 \mathrm{MeV}$ & $(34.8 \pm 1.5) \%$ \\
2 & $3556.20 \pm 0.09 \mathrm{MeV}$ & $459.28 \mathrm{MeV}$ & $1.97 \pm 0.11 \mathrm{MeV}$ & $(19.8 \pm 0.8) \%$ \\
\hline
\end{tabular}

Table 7.1: Selected properties of the $\chi_{c J}(1 P)$ states; all information is taken from [3]. The mass difference $\Delta m$ is defined as $\Delta m=m\left(\chi_{c J}\right)-m(J / \psi)$.

Contrary to the $\chi_{c J}(2 P)$ states, the $\chi_{c J}(1 P)$ states have been well studied by several different experiments and many of their fundamental properties are known to high precision [3], as listed in Table 7.1. The $\chi_{c J}$ states have large radiative decay widths $(\mathcal{O}(0.1 \mathrm{MeV}))$ and the branching fractions $\mathcal{B}\left(\chi_{c J} \rightarrow J / \psi \gamma\right)$ are large $(\mathcal{O}(10 \%))$ for the $\chi_{c 1}$ and $\chi_{c 2}$ states, but significantly smaller for the $\chi_{c 0}$ state (around 1\%) owing to its large hadronic decay width. The mass splittings between the three states are larger than in the $\chi_{b}$ system at between $46-96 \mathrm{MeV}$.

The $\chi_{c}$ states have been studied extensively by experiments at low energy $e^{+} e^{-}$ colliders, such as Crystall Ball and CLEO, typically running at a CM energy of $3.68 \mathrm{GeV}$ (the $\psi(2 S)$ resonance), where the $\chi_{c}$ states are readily produced in the radiative decays of the $\psi(2 S)$ (branching fractions of around 10\%), as shown in Figure 7.1. The $\chi_{c J}$ states were typically studied through their own radiative decays to $J / \psi, \chi_{c J} \rightarrow J / \psi \gamma$ (usually combined with $J / \psi \rightarrow \mu^{+} \mu^{-}$or $J / \psi \rightarrow e^{+} e^{-}$). The radiative decays $\psi(2 S) \rightarrow \chi_{c J} \gamma$ and $\chi_{c J} \rightarrow J / \psi \gamma$ have been measured to be 


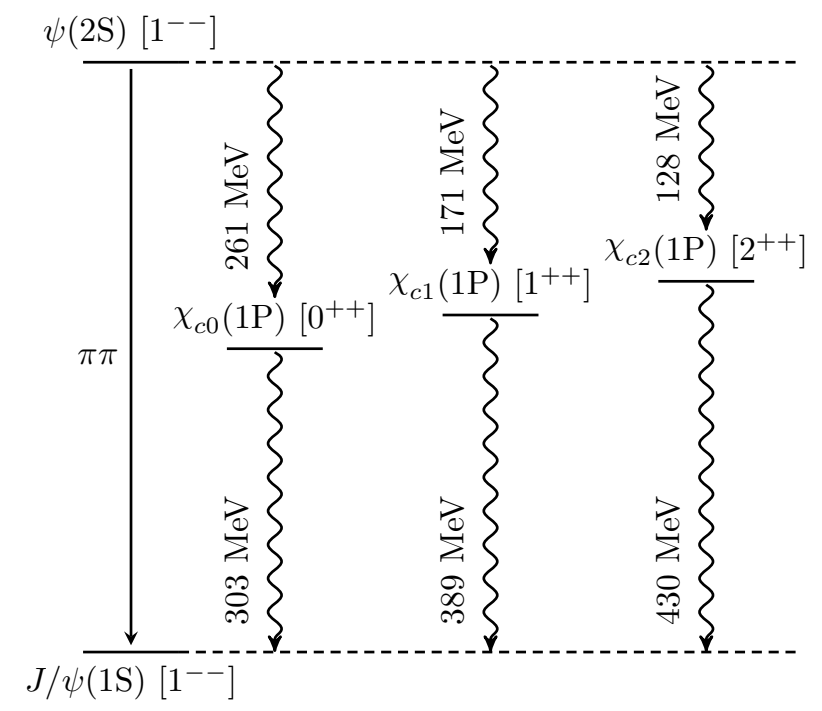

Figure 7.1: An energy-level diagram showing the allowed radiative transitions between the $J / \psi, \psi(2 S)$ and $\chi_{c J}$ states. Measurements of photon energies are taken from Ref. [3].

dominantly electric dipole (E1) transitions with small contributions (typically below $10 \%$ ) from magnetic quadrupole (M2) and electric octupole (E3) transitions [3].

The production of the $\chi_{c}$ states at hadron colliders represents an important facet of charmonium hadro-production phenomenology. In $p p$ collisions, the $\chi_{c}$ states can be produced directly in the primary parton-parton interaction and through feed-down from higher mass charmonium states (together referred to as prompt) or through the decays of $b$-hadrons (denoted non-prompt). The radiative decays of the $\chi_{c J}$ states, $\chi_{c J} \rightarrow J / \psi \gamma$, represent the most favourable channel to reconstruct the $\chi_{c J}$ states at hadron colliders due to their large branching fractions. The presence of a $J / \psi$ in the decay chain is also ideal for triggering through the decay $J / \psi \rightarrow \mu^{+} \mu^{-}$. This combination of decays is the typical choice for $\chi_{c}$ measurements at hadron colliders, though the decay $J / \psi \rightarrow e^{+} e^{-}$is also used (mainly at lower energy fixed target experiments).

Several measurements of $\chi_{c J}$ production have been performed at hadron collider experiments. Both CDF and D0 measured the contribution to the prompt $J / \psi$ production cross section from $\chi_{c J}$ feed-down decays, a quantity known as $R_{\chi_{c}}$, in 
$p \bar{p}$ collisions at $\sqrt{s}=1.8 \mathrm{TeV}$ (Tevatron Run I) [54,70]. CDF and D0 measured $p_{T}^{J / \psi}$ averaged fractional contributions of $0.297 \pm 0.017$ (stat.) \pm 0.057 (syst.) and $0.32 \pm 0.07$ (stat.) \pm 0.07 (syst.), respectively, for $p_{T}^{J / \psi}>4 \mathrm{GeV}$. CDF also measured this fraction as a function of $p_{T}^{J / \psi}$ and observed a decrease from around $31 \%$ at $p_{T}^{J / \psi}=$ $4 \mathrm{GeV}$ to around $26 \%$ for $p_{T}^{J / \psi}>10 \mathrm{GeV}$. Both analyses reconstructed photons from energy deposits measured with their respective electromagnetic calorimeters and observed a single unresolved $\chi_{c J}$ mass peak. Using the Tevatron Run I dataset, and later with early Run II data $(\sqrt{s}=1.96 \mathrm{TeV})$, CDF adopted a new approach to the reconstruction of $\chi_{c J}$ decays based on reconstructed $\gamma \rightarrow e^{+} e^{-}$conversions. The use of photon conversions, with their improved resolution, allowed the $\chi_{c 1}$ and $\chi_{c 2}$ states to be very well resolved, facilitating the first measurements of the relative production rates of the two states at a hadron collider $[38,148]$. These measurements provided the first evidence that the relative prompt production rates of the $\chi_{c 1}$ and $\chi_{c 2}$ states did not follow the $3: 5$ ratio expected from naive spin counting expectations and showed that more $\chi_{c 1}$ mesons are produced, relative to $\chi_{c 2}$, for $p_{T}^{J / \psi}>4 \mathrm{GeV}[38,148]$. The later CDF analysis also measured the relative nonprompt production rates of the $\chi_{c 1}$ and $\chi_{c 2}$ states [38].

The relative production rates of the promptly produced $\chi_{c 1}$ and $\chi_{c 2}$ states have been measured in $p p$ collisions at $\sqrt{s}=7 \mathrm{TeV}$ by LHCb (using both calorimetric [39] and conversion [149] approaches to photon reconstruction) and CMS (using converted photons) $[39,40,149]$. These three measurements confirmed the behaviour first observed by CDF and considerably extended the measured range in $p_{T}^{J / \psi}$, while also offering improved precision. The fraction of prompt $J / \psi$ produced in feed-down from $\chi_{c J}$ decays has also been measured by LHCb in $p p$ collisions at $\sqrt{s}=7 \mathrm{TeV}$. The measurements show a strong $p_{T}^{J / \psi}$ dependence for the region $2.0<y^{J / \psi}<4.5$, with the measured fraction varying from around $14 \%$ at $p_{T}^{J / \psi}=2 \mathrm{GeV}$ to around $26 \%$ at $p_{T}^{J / \psi}=14 \mathrm{GeV}$. No measurements of the non-prompt production of the $\chi_{c J}$ states have yet been performed at the LHC. 


\subsection{Measurement Overview}

Throughout the remainder of this chapter, a measurement of $\chi_{c 1}$ and $\chi_{c 2}$ production in $p p$ collisions at $\sqrt{s}=7 \mathrm{TeV}$ will be described. The conventional decay mode choice, adopted at most previous hadron collider experiments, is also chosen to perform this analysis; $\chi_{c J} \rightarrow J / \psi \gamma$ coupled with $J / \psi \rightarrow \mu^{+} \mu^{-}$. In addition to the large overall branching fractions, the ATLAS detector is capable of triggering upon $J / \psi \rightarrow \mu^{+} \mu^{-}$events (as described in Section 5.5) and can also reconstruct the low transverse momentum final state of $\mu^{+} \mu^{-} \gamma$ with sufficient efficiency and precision. In order to reconstruct photons with a precision sufficient to allow the individual $\chi_{c 1}$ and $\chi_{c 2}$ states to be resolved, photons are reconstructed from $\gamma \rightarrow e^{+} e^{-}$conversions in the ATLAS ID, as discussed in Section 5.3. This method provides the momentum resolution necessary to resolve the individual states and also has an efficiency sufficiently high to allow a large sample of $\chi_{c J}$ decays to be reconstructed from the data sample used in the analysis. The pseudo-proper decay time variable $\tau$, discussed in Section 5.6.2, is used to distinguish between prompt and non-prompt $\chi_{c}$, allowing the individual prompt and non-prompt cross sections to be measured for the $\chi_{c 1}$ and $\chi_{c 2}$ states. While a sample of $\chi_{c 1}$ and $\chi_{c 2}$ decays large enough to perform a reliable cross section measurement can be reconstructed from the data sample used, the number of reconstructed $\chi_{c 0}$ decays within the same sample is significantly lower, owing to its low radiative branching fraction and a kinematic acceptance lower than the $\chi_{c 1}$ and $\chi_{c 2}$ states (due to the softer photon energy in the decay). While some indication for the $\chi_{c 0}$ state is observed, no attempt is made to measure its production cross section due these limitations.

The aim of this analysis is to measure the prompt and non-prompt production cross sections for the $\chi_{c 1}$ and $\chi_{c 2}$ states as a function of both $p_{T}^{J / \psi}$ and $p_{T}^{\chi_{c}}$. These measurements also allow several cross section ratios to be derived and can be combined with the ATLAS measurements in Ref. [77] to determine the fraction of 
prompt $J / \psi$ produced in feed-down from $\chi_{c J}$ decays, $R_{\chi_{c}}$.

\subsection{Data and MC Simulation Samples}

The analysis is performed with a sample representing $4.5 \mathrm{fb}^{-1}$ of $\sqrt{s}=7 \mathrm{TeV} p p$ collision data collected by the ATLAS experiment during the 2011 LHC run. Events are only considered for analysis if they were recorded under stable LHC beam conditions with all relevant detector sub-systems fully operational. The data sample used was collected with a di-muon trigger with a $4 \mathrm{GeV}$ muon transverse momentum threshold, known as EF_2mu4_Jpsimumu, designed to select events containing a $J / \psi \rightarrow \mu^{+} \mu^{-}$decay, as described in Section 5.5. During the latter half of the 2011 LHC run, the beam parameters of the LHC were modified such that the average instantaneous luminosity delivered to ATLAS increased by around $50 \%$. To cope with this, the requirements of the EF_2mu4_Jpsimumu trigger were modified to reduce its acceptance rate. The original trigger did not require a minimum muon transverse momentum at L1, but applied the $4 \mathrm{GeV}$ requirement only at the HLT level. The modified trigger required that the muon satisfy the $4 \mathrm{GeV}$ requirement directly at L1. Given the limited granularity information available at L1, the efficiency of the modified trigger was slightly reduced with respect to the original trigger. This modified trigger, known as EF_2mu4T_Jpsimumu, replaced the previous trigger (with no overlap) and was used to collect around one half of the total data sample.

Several simulated $\chi_{c J} \rightarrow J / \psi \gamma$ event samples (with $J / \psi \rightarrow \mu^{+} \mu^{-}$for each event) are used in various aspects of the analysis. All of the event samples are generated with the PYTHIA6 MC event generator [99]. Three independent samples of directly produced (prompt) $\chi_{c J}$ events are used (one sample for each of $J=0,1,2$ ) in addition to three independent samples of $\chi_{c J}$ events produced in the decays of $b$-hadrons (non-prompt) (one sample for each of $J=0,1,2$ ). Each sample contains around $10^{6}$ events. The events are filtered at the event generation stage, such that 
events are only retained if the transverse momentum of each muon from the $J / \psi \rightarrow$ $\mu^{+} \mu^{-}$decay is greater than $3.75 \mathrm{GeV}$ and the photon from the $\chi_{c J}$ decay has a transverse momentum greater than $0.9 \mathrm{GeV}$. These filter requirements are motivated by the fact that a muon transverse momentum requirement of $4 \mathrm{GeV}$ is imposed by the trigger used to perform the analysis. The transverse momentum requirement on the photon is chosen to be slightly below the minimum transverse momentum with which photons can be reconstructed (enough to account for detector resolution).

Each sample of simulated $p p$ events is propagated through the ATLAS detector simulation and processed with the same reconstruction algorithms used to process the data, as described in Section 4.7.

\subsection{Reconstruction of $\chi_{c J} \rightarrow J / \psi \gamma$ decays}

Events are first selected in which either the EF_2mu4_Jpsimumu (in the early data) or the EF_2mu4T_Jpsimumu (in the later data) trigger was fired. These events are then analysed to search for reconstructed di-muon pairs consistent with $J / \psi \rightarrow \mu^{+} \mu^{-}$ decays. The subset of the triggered events that contain a reconstructed $J / \psi \rightarrow \mu^{+} \mu^{-}$ candidate is analysed further to search for a converted photon consistent with being produced in the decay $\chi_{c J} \rightarrow J / \psi \gamma$. 


\subsubsection{Selection of $J / \psi \rightarrow \mu^{+} \mu^{-}$decays}

Di-muon pairs consistent with the decay $J / \psi \rightarrow \mu^{+} \mu^{-}$seed the reconstruction of $\chi_{c J} \rightarrow J / \psi \gamma$ decays. Events are selected that contain two muon objects, reconstructed with the combined approach, using the Staco algorithm described in Section 5.2. Reconstructed di-muon pairs are required to satisfy the following requirements:

- The two muons must have opposite charges.

- Each muon must be reconstructed from an inner detector track matched to a track in the muon spectrometer.

- The pseudorapidity of each muon must satisfy $\left|\eta^{\mu}\right|<2.3$.

- Each muon must have transverse momentum $p_{T}^{\mu}>4 \mathrm{GeV}$.

In addition to these requirements, the inner detector track associated with each muon candidate is required to satisfy a series of quality requirements:

- The track should contain at least one pixel B layer (the first layer) hit, if such a hit is expected.

- The sum of the number of pixel hits and the number of crossed dead pixel sensors must be greater than 1 .

- The sum of the number of SCT hits and the number of crossed dead SCT sensors for the track must be greater than 5 .

- The number of crossed pixel and SCT holes must be less than 3.

- The track reconstructed in the silicon detectors must have a successful TRT extension if expected. 
The di-muon pair reconstructed offline is required to match spatially the two muon objects reconstructed by the HLT (within $\Delta R=\sqrt{(\Delta \phi)^{2}+(\Delta \eta)^{2}}<0.01$ ). Each pair of muons that passes these requirements is fitted to a common vertex with no kinematic constraints applied. The fit is required to converge with $\chi^{2} /[$ d.o.f. $]<$ 200, a requirement which is fully efficient for genuine $J / \psi \rightarrow \mu^{+} \mu^{-}$decays [87]. Dimuon pairs satisfying these requirements are considered as candidate $J / \psi \rightarrow \mu^{+} \mu^{-}$ decays if they have a di-muon invariant mass within $2.95<m\left(\mu^{+} \mu^{-}\right)<3.25 \mathrm{GeV}$. This invariant mass requirement retains $99.0 \pm 0.5 \%$ of $J / \psi \rightarrow \mu^{+} \mu^{-}$decays. Finally, a fiducial region of $\left|y^{\mu^{+} \mu^{-}}\right|<0.75$ is chosen to coincide with the selection used in the ATLAS measurement of $J / \psi$ production [77]. This central region is used as it exhibits a di-muon invariant mass resolution which is sufficient to resolve the individual $\chi_{c 1}$ and $\chi_{c 2}$ states. The overall mass resolution deteriorates quickly beyond this region and a reliable separation of the two states becomes progressively more difficult to achieve. 


\subsubsection{Selection of Photon Conversions}

Photon conversions to an $e^{+} e^{-}$pair are reconstructed from ID tracks alone, with no input from the calorimeters, using the conversion finding algorithm described in Section 5.3.1. In this analysis, only tracks reconstructed from hits in the silicon tracking detectors are used (though such tracks often also contain TRT hits). This approach provides a sample of reconstructed conversions with a purity and momentum resolution superior to that obtained by including tracks reconstructed from TRT information alone, albeit at the expense of some efficiency. Photon conversions are selected for further analysis if they satisfy the following requirements:

- The two tracks must have opposite charges.

- Each track must contain at least six hits in the SCT layers of the ID (no additional requirements on pixel or TRT hits are imposed).

- The fitting of the two tracks to a common conversion vertex must converge with $\chi^{2} /[$ d.o.f. $]<5$.

- The transverse momentum of each track must satisfy $p_{T}>0.4 \mathrm{GeV}$.

- The transverse momentum of the reconstructed conversion must satisfy $p_{T}^{\gamma}>$ $1.5 \mathrm{GeV}$.

- The pseudorapidity of the reconstructed conversion must satisfy $\left|\eta^{\gamma}\right|<2.0$.

- The radial position of the reconstructed conversion vertex, $R$, must satisfy $40<R<150 \mathrm{~mm}$.

The requirement on the radial position of reconstructed conversion selects those reconstructed within the three silicon pixel layers of the ID. Conversions reconstructed in this region exhibit an optimal reconstruction efficiency, with all SCT and TRT layers available to reconstruct the $e^{+} e^{-}$tracks. The reconstruction efficiency and purity decreases progressively for conversions reconstructed at ever larger 
radii as more active tracking layers are traversed before the photon converts. The requirement that the reconstructed conversion vertex be displaced radially by at least $40 \mathrm{~mm}$ from the origin removes conversions occurring within the beam pipe material, which are more contaminated by fake conversions from promptly produced $e^{+} e^{-}$pairs (typically from the decay $\pi^{0} \rightarrow e^{+} e^{-} \gamma$ ) than conversions reconstructed in the pixel layers. The minimum transverse momentum requirement of $p_{T}^{\gamma}>1.5 \mathrm{GeV}$ is used to ensure that photons used in the measurement are within a region where the acceptance for $\chi_{c J} \rightarrow J / \psi \gamma$ decays is high and not rapidly changing, given the $p_{T}^{\mu}>4 \mathrm{GeV}$ requirement imposed by the trigger.

Each reconstructed photon conversion which satisfies these requirements is paired with every $J / \psi \rightarrow \mu^{+} \mu^{-}$candidate in the same event to form loose $\chi_{c J} \rightarrow J / \psi \gamma$ candidates.

\subsubsection{Selection of $\chi_{c J} \rightarrow J / \psi \gamma$ decays}

Loose $\chi_{c J} \rightarrow J / \psi \gamma$ candidates, consisting of a reconstructed $J / \psi \rightarrow \mu^{+} \mu^{-}$decay and a photon conversion are subjected to some additional selection requirements to reject $J / \psi \gamma$ pairs that are not consistent with originating from a $\chi_{c J} \rightarrow J / \psi \gamma$ decay. The large number of additional $p p$ interactions in each event (pileup), shown in Figure 4.12 to be between around 2 and 20 for the data sample used, can lead to a large number of $J / \psi \gamma$ pairs in which the $J / \psi$ and photon are produced in different $p p$ interactions and not from the decay of a $\chi_{c J}$. The extrapolated trajectory of a well reconstructed converted photon produced in a $\chi_{c J} \rightarrow J / \psi \gamma$ decay should intersect the $J / \psi \rightarrow \mu^{+} \mu^{-}$vertex within experimental resolution. In general, this should not be the case for uncorrelated $J / \psi \gamma$ pairs that originate from separate $p p$ interactions. This "intersection" of the photon trajectory with the $J / \psi \rightarrow \mu^{+} \mu^{-}$vertex can be quantified with the 3 -dimensional impact parameter $a_{0}$, which is calculated from the converted photon's vertex and momentum in addition to the $J / \psi \rightarrow \mu^{+} \mu^{-}$vertex, as described in Section 5.6.3. The distribution of $a_{0}$ for genuine $\chi_{c J} \rightarrow J / \psi \gamma$ 
decays peaks at zero, while the distribution for $J / \psi \gamma$ pairs produced in separate $p p$ collisions is very broad. A requirement of $a_{0}<5 \mathrm{~mm}$ is found to have a negligible inefficiency for genuine $\chi_{c J} \rightarrow J / \psi \gamma$ decays but is effective in removing many of the background $J / \psi \gamma$ pairs due to pileup, as shown in Figure 7.2. $J / \psi \gamma$ pairs which satisfy the $a_{0}<5 \mathrm{~mm}$ requirement are considered as $\chi_{c J} \rightarrow J / \psi \gamma$ candidates and are retained for further analysis.

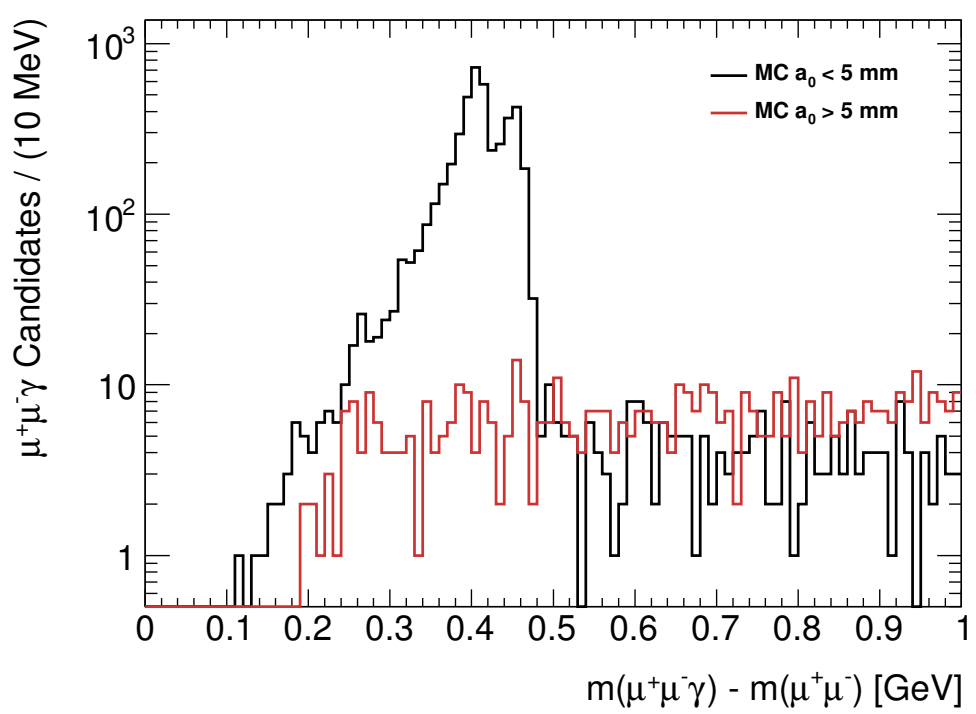

Figure 7.2: The mass difference distribution $m\left(\mu^{+} \mu^{-} \gamma\right)-m\left(\mu^{+} \mu^{-}\right)$for $\chi_{c 1}$ and $\chi_{c 2}$ candidates reconstructed from the MC simulation samples described in Section 7.3. The black histogram shows reconstructed $\chi_{c J} \rightarrow J / \psi \gamma$ candidates which satisfy the requirement $a_{0}<5 \mathrm{~mm}$ while the red histogram shows candidates which fail this requirement. No evidence for $\chi_{C J}$ peaks is observed in the sample which fail the requirement. 


\subsection{Cross Section Measurement Procedure}

Differential cross sections for both prompt and non-prompt $\chi_{c 1}$ and $\chi_{c 2}$ production are measured both as functions of $J / \psi$ transverse momentum, $p_{T}^{J / \psi}$, and of $\chi_{c}$ transverse momentum, $p_{T}^{\chi_{c}}$. The differential cross section, in transverse momentum $p_{T}$ (where $p_{T}$ is either $p_{T}^{\chi_{c}}$ or $p_{T}^{J / \psi}$ ), for prompt $(\mathrm{P})$ and non-prompt (NP) $\chi_{c}$ production is determined from,

$$
\frac{d \sigma^{\mathrm{P}(\mathrm{NP})}\left(\chi_{c J}\right)}{d p_{T}} \times \mathcal{B}\left(\chi_{c J} \rightarrow J / \psi \gamma\right) \times \mathcal{B}\left(J / \psi \rightarrow \mu^{+} \mu^{-}\right)=\frac{N_{J}^{\mathrm{P}(\mathrm{NP})}}{\mathcal{L} \times \Delta p_{T}}
$$

where $N_{J}^{\mathrm{P}(\mathrm{NP})}$ is the acceptance- and efficiency-corrected prompt (non-prompt) $\chi_{c J}$ signal yield in a given transverse momentum bin, $\mathcal{L}$ is the integrated luminosity of the data sample and $\Delta p_{T}$ is the bin width in transverse momentum.

Each reconstructed $\chi_{c J}$ candidate is assigned a weight, $w$, to correct for experimental losses due to finite acceptance and reconstruction efficiency. The percandidate weight is factorised into several individual components,

$$
w^{-1}=\mathcal{A} \cdot \epsilon_{\text {trig }} \cdot \epsilon_{\text {di-muon }} \cdot \epsilon_{\gamma},
$$

where $\mathcal{A}$ is the acceptance for the candidate and $\epsilon_{\text {trig }}, \epsilon_{\text {di-muon }}$ and $\epsilon_{\gamma}$ are the trigger efficiency, $J / \psi \rightarrow \mu^{+} \mu^{-}$reconstruction efficiency and total converted photon reconstruction efficiency for the candidate. The corrected yields $N_{J}^{\mathrm{P}(\mathrm{NP})}$ are then obtained from a weighted, simultaneous fit to the mass difference, $\Delta m=$ $m\left(\mu^{+} \mu^{-} \gamma\right)-m\left(\mu^{+} \mu^{-}\right)$, and pseudo-proper decay time, $\tau$, distributions. The mass difference distribution is used to discriminate between $\chi_{c}$ signal candidates and backgrounds in place of the three-body invariant mass $m\left(\mu^{+} \mu^{-} \gamma\right)$. The effective mass resolution observed in the $\Delta m$ distribution is superior to that of $m\left(\mu^{+} \mu^{-} \gamma\right)$ due to a partial cancellation of contributions from the di-muon invariant mass, as described 
in Section 5.6, and provides the mass resolution necessary to separate the individual $\chi_{c 1}$ and $\chi_{c 2}$ states.

The calculation of each individual contribution to the per-candidate weight is described in the remainder of this section.

\subsubsection{Acceptance}

The per-candidate acceptance is defined as the probability for all of the final state decay products in a $\chi_{c J} \rightarrow J / \psi \gamma \rightarrow \mu^{+} \mu^{-} \gamma$ decay to fall within the fiducial region of the detector (i.e. the region within which the total reconstruction efficiency is nonzero). The fiducial region for muons is defined as $p_{T}^{\mu}>4 \mathrm{GeV}$ and $\left|\eta^{\mu}\right|<2.3$, while the fiducial region for converted photons is defined as $p_{T}^{\gamma}>1.5 \mathrm{GeV}$ and $\left|\eta^{\gamma}\right|<2.0$. The per-candidate acceptance depends on the kinematics of the original $\chi_{c J}$ and of its decay products. The acceptances for $\chi_{c 1}$ and $\chi_{c 2}$ are not identical, primarily due to their different masses, which causes the kinematic distributions of the final state decay products to differ.

The acceptance also has a strong dependence on the angular distributions of the final state decay products in their respective decay frames. The analytical form of these angular distributions is a function of the polarisation of the $\chi_{c J}$ states, measured with respect to a given polarisation axis. The angular distribution of the $\mu^{+}$in the $J / \psi$ rest frame takes the form,

$$
W(\theta, \phi) \propto \frac{1}{3+\lambda_{\theta}}\left[1+\lambda_{\theta} \cos ^{2} \theta+\lambda_{\phi} \sin ^{2} \theta \cos 2 \phi+\lambda_{\theta \phi} \sin 2 \theta \cos \phi\right]
$$

where $\theta$ and $\phi$ are the polar and azimuthal angles respectively between the $\mu^{+}$ direction in the $J / \psi$ rest frame and the chosen polarisation axis [62]. The angular distribution of the $J / \psi$ in the $\chi_{c J}$ rest frame takes the same form, 


$$
W(\Theta, \Phi) \propto \frac{1}{3+\lambda_{\Theta}}\left[1+\lambda_{\Theta} \cos ^{2} \Theta+\lambda_{\Phi} \sin ^{2} \Theta \cos 2 \Phi+\lambda_{\Theta \Phi} \sin 2 \Theta \cos \Phi\right]
$$

where $\Theta$ and $\Phi$ are the polar and azimuthal angles respectively between the $J / \psi$ direction in the $\chi_{c J}$ rest frame and the chosen polarisation axis [62]. These angular distributions are valid for $\chi_{c 1}$ in general and also valid of $\chi_{c 2}$ if the radiative decay $\chi_{c 2} \rightarrow J / \psi \gamma$ is modelled as a pure electric dipole, E1, transition and contributions from higher order multipoles are neglected. This is a well-motivated approximation, given that the world average data on the multipole structure of $\chi_{c 1,2} \rightarrow J / \psi \gamma$ decay suggests that higher-order contributions represent less than $10 \%$ of the total transition amplitude [3]. The $\lambda$ coefficients in Equations 7.3 and 7.4 are functions of the $\chi_{c J}$ polarisation with respect to a chosen axis. It has been shown that the form of the angular distributions in Equations 7.3 and 7.4 are identical $\left(\lambda_{\theta}=\lambda_{\Theta}\right.$, $\left.\lambda_{\phi}=\lambda_{\Phi}, \lambda_{\theta \phi}=\lambda_{\Theta \Phi}\right)$ if the angles $\theta, \phi$ and $\Theta, \Phi$ are measured with respect to a parallel polarisation axes [62].

Unfortunately, the polarisation of the $\chi_{c J}$ states produced at the LHC has not been measured and no other measurements have been made under comparable experimental conditions (e.g. at the Tevatron). This lack of knowledge gives rise to an irreducible uncertainty in the acceptance. To quantify the dependence of the acceptance on the polarisation of the $\chi_{c J}$ states, the polarisation axis is chosen as the $\chi_{c J}$ direction of propagation in the laboratory frame, also known as the helicity (HX) axis. Several polarisation scenarios are identified that represent the extremes of the allowed values of the $\lambda$ parameters which also give rise to the largest variations in the acceptance. The values of the $\lambda$ parameters for the scenarios studied are shown in Table 7.2. These scenarios span the allowed regions of the $\lambda_{\theta}-\lambda_{\phi}$ plane, as shown in Figure 7.3, and include the pure helicity states of the $\chi_{c 1}$ and $\chi_{c 2}$. Scenarios involving non-zero values of $\lambda_{\theta \phi}$ are found to give rise to variations 
in the acceptance that are significantly smaller than the variations observed for the scenarios shown in Table 7.2.

\begin{tabular}{|c|c|c|c|c|}
\hline & Label & $\lambda_{\theta}$ & $\lambda_{\phi}$ & $\lambda_{\theta \phi}$ \\
\hline \multirow{6}{*}{$\chi_{c 1}$} & Isotropic & 0 & 0 & 0 \\
& Helicity 0 & +1 & 0 & 0 \\
& Helicity \pm 1 & $-1 / 3$ & 0 & 0 \\
& AZ+ & $-1 / 3$ & $+1 / 3$ & 0 \\
& AZ- & $-1 / 3$ & $-1 / 3$ & 0 \\
\hline \multirow{6}{*}{$\chi_{c 2}$} & Isotropic & 0 & 0 & 0 \\
& Helicity 0 & $-3 / 5$ & 0 & 0 \\
& Helicity \pm 1 & $-1 / 3$ & 0 & 0 \\
& Helicity \pm 2 & +1 & 0 & 0 \\
& AZ + & $+1 / 5$ & $+1 / \sqrt{5}$ & 0 \\
& $\mathrm{AZ}-$ & $+1 / 5$ & $-1 / \sqrt{5}$ & 0 \\
\hline
\end{tabular}

Table 7.2: The set of $\chi_{c 1}$ and $\chi_{c 2}$ polarisation scenarios studied to quantify the uncertainty on the acceptance due to the unknown $\chi_{c J}$ polarisation.

The central value for the acceptance is calculated assuming isotropic decay angular distributions $\left(\lambda_{\theta}=\lambda_{\phi}=\lambda_{\theta \phi}=0\right)$ and the alternative scenarios shown in Table 7.2 are used to calculate an uncertainty envelope.

The acceptance corrections are calculated using a generator level MC simulation, which generates a large number of $\chi_{c 1}$ and $\chi_{c 2}$ decays, $\chi_{c J} \rightarrow J / \psi \gamma \rightarrow \mu^{+} \mu^{-} \gamma$, and calculates the fraction of these decays that fall within the fiducial region to provide an estimate of the acceptance. Each generated event is given a weight such that the angular distributions of the simulated decays take the form of the angular distributions shown in Equations 7.3 and 7.4 for a given polarisation scenario.

Two separate acceptance corrections are calculated to be used in the measurements of cross sections binned in $p_{T}^{\chi_{c}}$ and $p_{T}^{J / \psi}$. The acceptance correction to be used in the measurement of cross sections binned in $p_{T}^{\chi_{c}}$ is parametrised as an acceptance map binned in $p_{T}^{\chi_{c}}$ and $\left|y^{\chi_{c}}\right|$. This acceptance map is independent of the shape of the $p_{T}^{\chi_{c}}$ distribution and requires no a priori knowledge to calculate. The acceptance maps binned in $p_{T}^{\chi_{c}}$ and $\left|y^{\chi_{c}}\right|$, for all the scenarios considered, are shown in Figure 7.4 for $\chi_{c 1}$ and Figure 7.5 for $\chi_{c 2}$. 


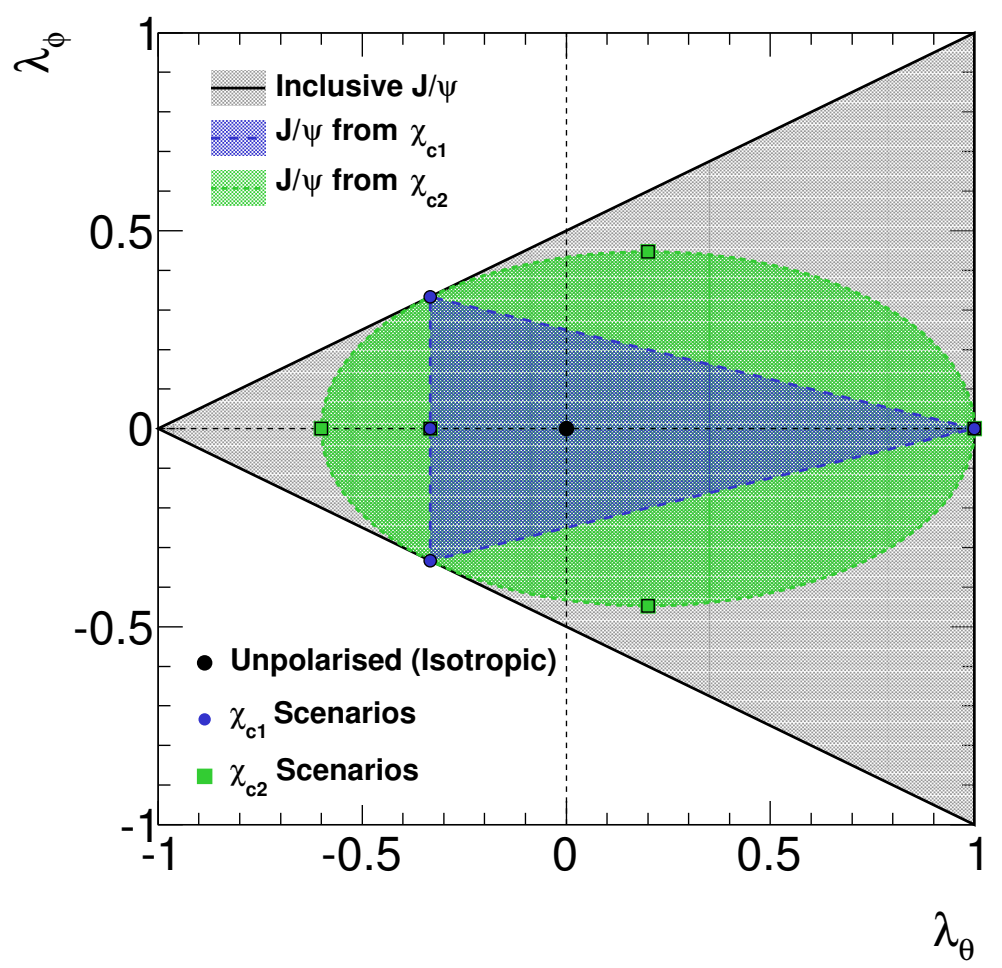

Figure 7.3: The allowed values of the $\lambda_{\theta}$ and $\lambda_{\phi}$ coefficients for inclusive $J / \psi$ production (grey) and for $J / \psi$ produced in radiative $\chi_{c 1}$ (blue) and $\chi_{c 2}$ (green) decays. Information is taken from Ref. [63].

Given the lack of any knowledge of $\chi_{c J}$ polarisation, the isotropic scenario is chosen as the central value. This lies between the extreme scenarios and has also been adopted in all previous measurements of $\chi_{c J}$ production at hadron colliders, and so allows direct comparisons to be made between measurements [38-40, 54, 82, 149].

The acceptance correction to be used in the measurement of cross sections binned in $p_{T}^{J / \psi}$ is parametrised as a correction binned in $p_{T}^{J / \psi}$. A $J / \psi$ produced in a $\chi_{c J} \rightarrow$ $J / \psi \gamma$ decay with a given $p_{T}^{J / \psi}$ can be produced from the decay of a $\chi_{c J}$ with a range of different $p_{T}^{\chi_{c}}$. As a consequence of this, the acceptance correction depends on the transverse momentum distribution of the simulated $\chi_{c J}$ decays and can not be calculated without an assumption or measurement of this distribution. Rather than making any assumption about this distribution, an analytical parametrisation of the $p_{T}^{\chi_{c}}$ distribution is fitted to the fully corrected $p_{T}^{\chi_{c}}$ distribution measured in this analysis and this is used as an input to the simulation (i.e. the cross sections binned 
in $p_{T}^{\chi_{c}}$ are measured first and fed back into simulation to facilitate the measurement of the cross sections binned in $\left.p_{T}^{J / \psi}\right)$. The acceptance corrections binned in $p_{T}^{J / \psi}$ are shown in Figure 7.6.

The experimental resolution in $p_{T}^{\chi_{c}}$, described in detail in Section 7.7, is asymmetric due to electron energy losses. The consequence of this asymmetry is that slightly more $\chi_{c J} \rightarrow J / \psi \gamma$ candidates are reconstructed with $p_{T}^{\chi_{c}}$ lower, rather than higher, than the "true" value of $p_{T}^{\chi c}$. This can result in a bias when applying the acceptance correction binned in $p_{T}^{\chi_{c}}$, based on the reconstructed $p_{T}^{\chi_{c}}$, since the percandidate acceptance will (on average) be too low, resulting in an over-correction of the measurement. To compensate for this effect, the reconstructed $p_{T}^{\chi_{c}}$ for each $\chi_{c J}$ is scaled by 1.006 (derived from the reciprocal of the mean value of the $p_{T}^{\chi_{c}}$ resolution function shown in Figure 7.21) before evaluating the per-candidate acceptance (this scaling is used only for this purpose). Simulation studies show that this procedure removes the majority of the bias, with any residual variations being bounded by $\pm 2 \%$. The acceptance corrections binned in $p_{T}^{J / \psi}$ do not experience a similar bias since the experimental resolution in $p_{T}^{J / \psi}$ is consistent with being symmetric (see Figure 7.21). 


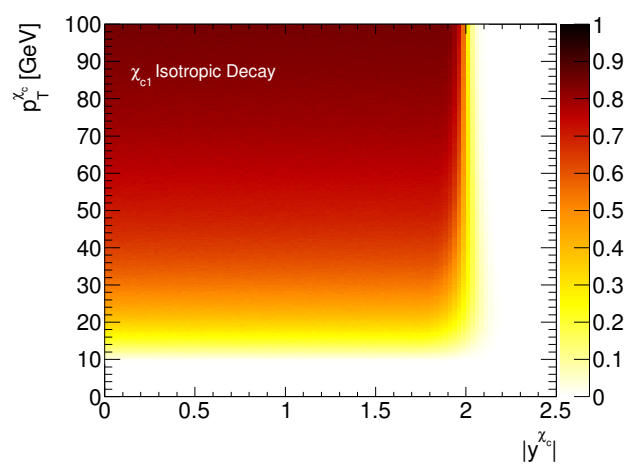

(a) Isotropic

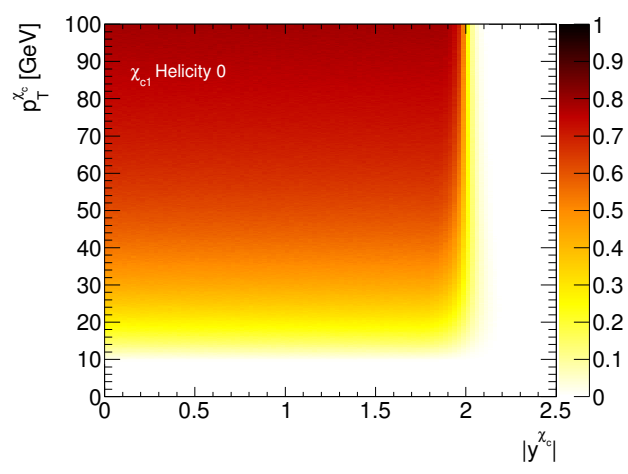

(b) Helicity 0

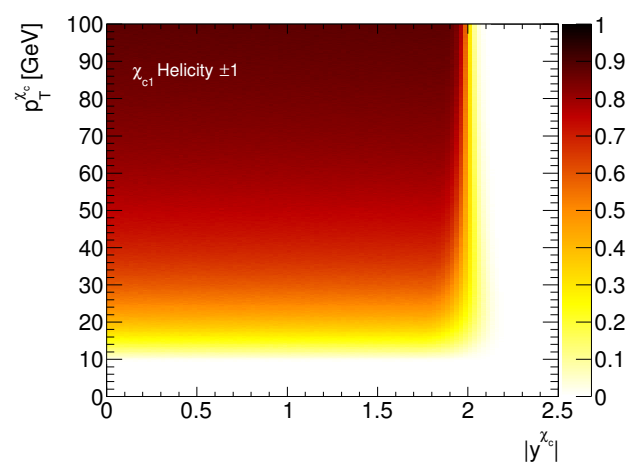

(c) Helicity \pm 1

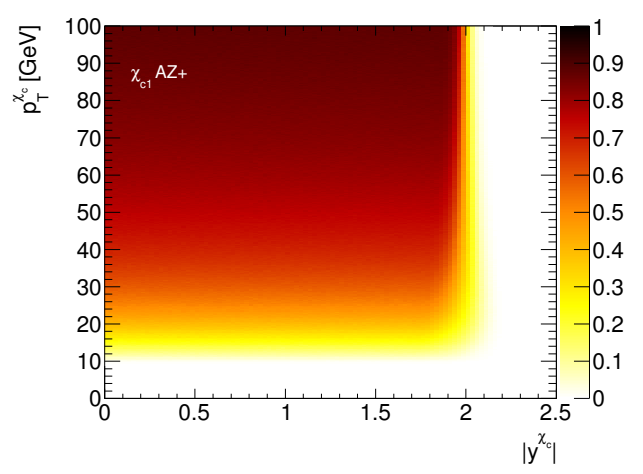

(d) $\mathrm{AZ}+$

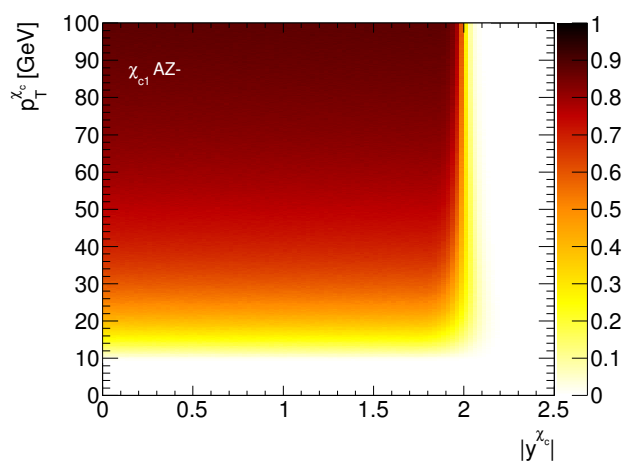

(e) $\mathrm{AZ}-$

Figure 7.4: Acceptance maps binned in $p_{T}^{\chi_{c}}$ and $\left|y^{\chi_{c}}\right|$ for $\chi_{c 1} \rightarrow J / \psi \gamma \rightarrow \mu^{+} \mu^{-} \gamma$ decays, calculated for each of the polarisation scenarios considered. 


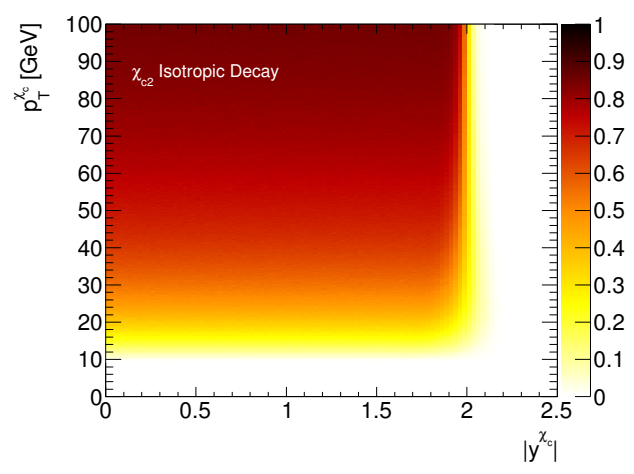

(a) Isotropic

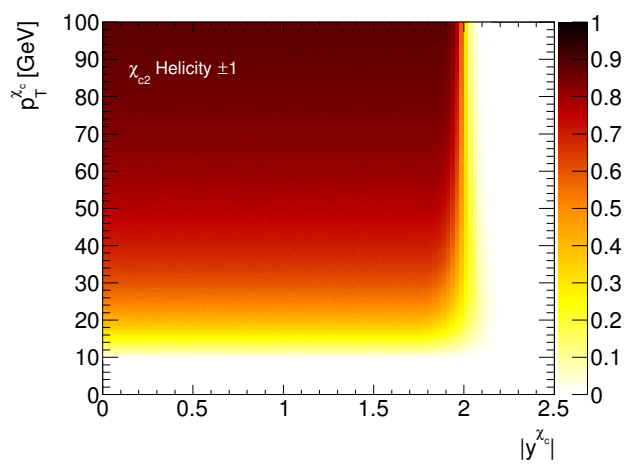

(c) Helicity \pm 1

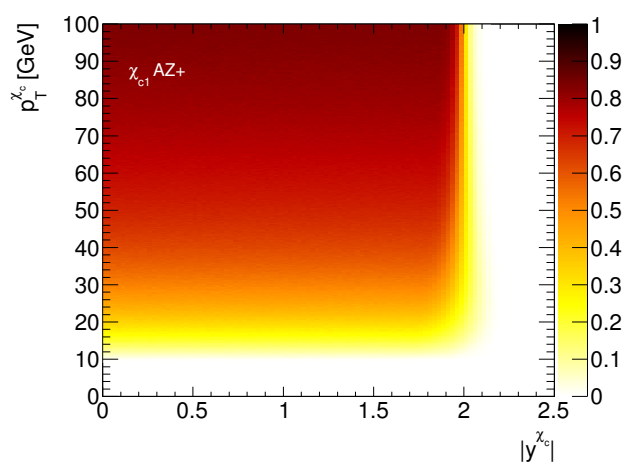

(e) $\mathrm{AZ}+$

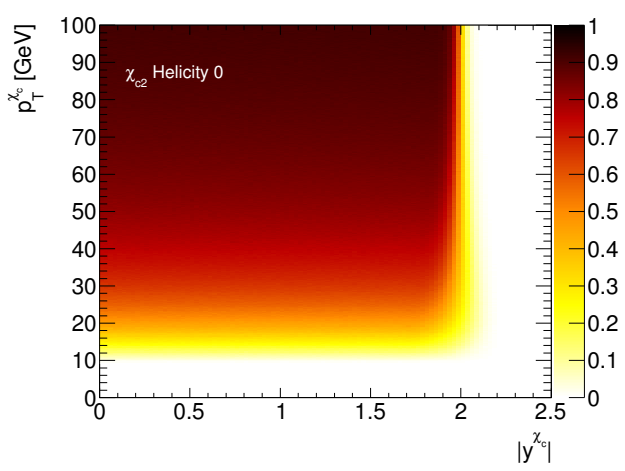

(b) Helicity 0

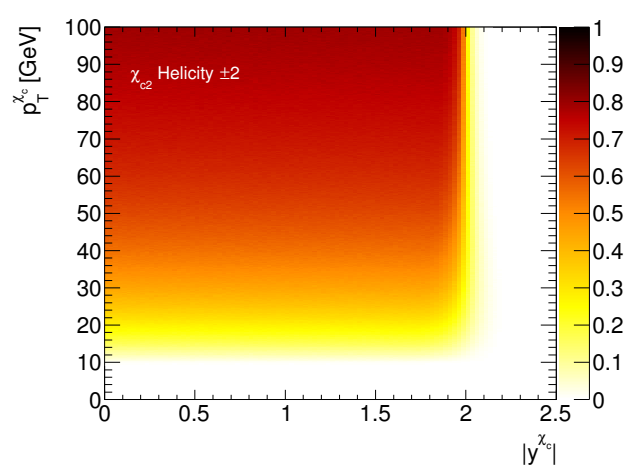

(d) Helicity \pm 2

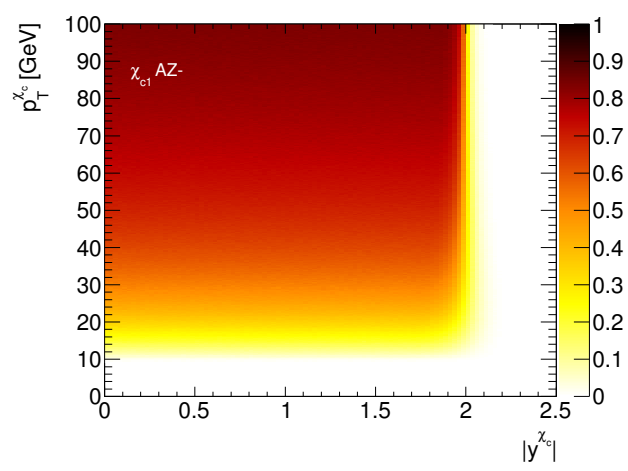

(f) $\mathrm{AZ}-$

Figure 7.5: Acceptance maps binned in $p_{T}^{\chi_{c}}$ and $\left|y^{\chi_{c}}\right|$ for $\chi_{c 2} \rightarrow J / \psi \gamma \rightarrow \mu^{+} \mu^{-} \gamma$ decays, calculated for each of the polarisation scenarios considered. 


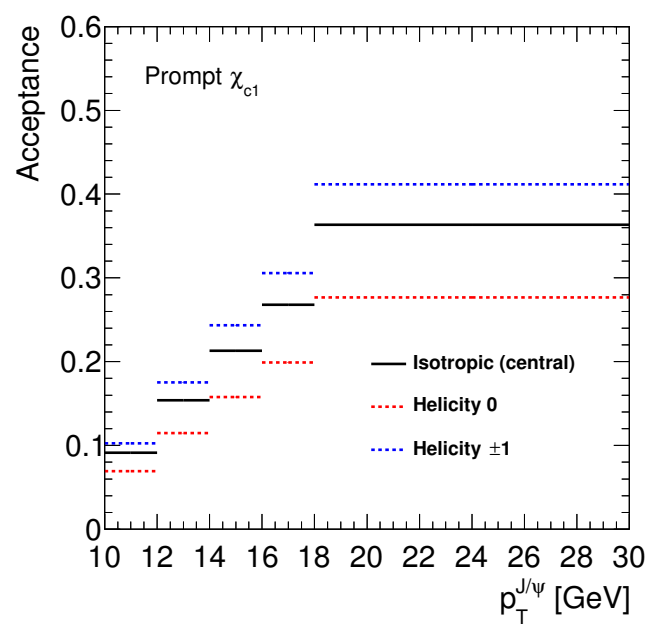

(a) Prompt $\chi_{c 1}$

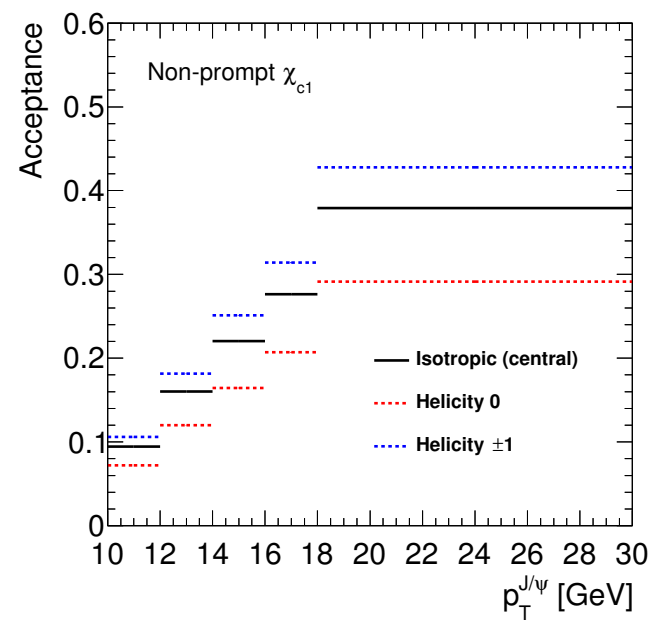

(c) Non-prompt $\chi_{c 1}$

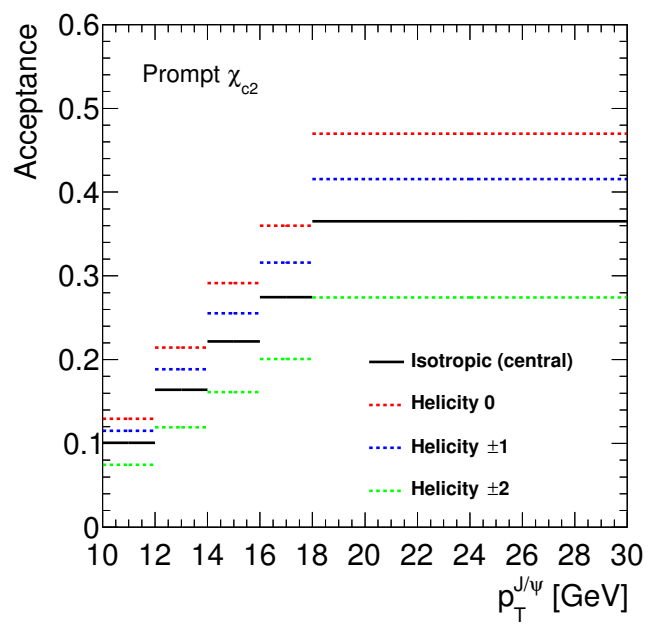

(b) Prompt $\chi_{c 2}$

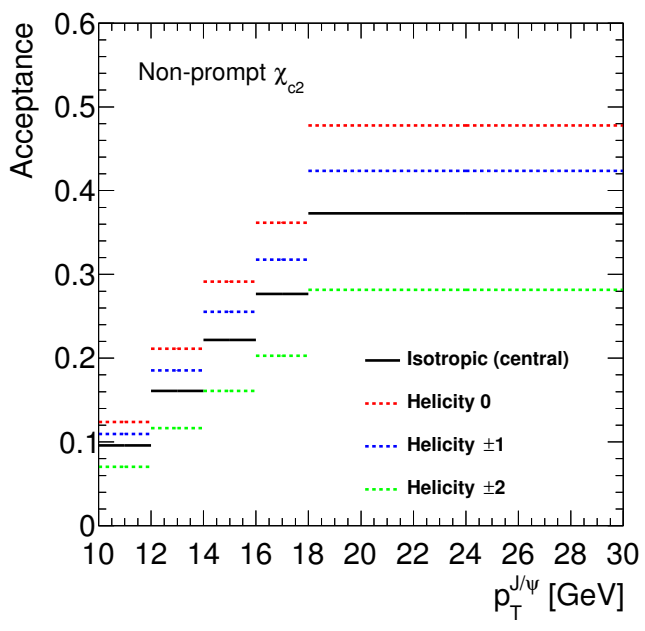

(d) Non-prompt $\chi_{c 2}$

Figure 7.6: Acceptance corrections binned in $p_{T}^{J / \psi}$ for $\chi_{c 1}(\mathrm{a}, \mathrm{c})$ and $\chi_{c 2}(\mathrm{~b}, \mathrm{~d})$, for isotropic decay angular distributions (central value) and for the helicity $0, \pm 1, \pm 2$ scenarios. The scenarios which include azimuthal anisotropy (AZ \pm ) are not shown, as for $\chi_{c 1}$ they closely follow the helicity \pm 1 scenarios while for $\chi_{c 2}$ they closely follow the isotropic central values. 


\subsubsection{Trigger Efficiency}

The di-muon trigger efficiency is defined as the efficiency with which the ATLAS trigger system (or more specifically the EF_2mu4(T)_Jpsimumu algorithm) can select events which contain a di-muon candidate that satisfies all of the offline requirements described in Section 7.4.1. The approach discussed here is identical to the method described in [87], which uses a sample of $J / \psi \rightarrow \mu^{+} \mu^{-}$and $\Upsilon(n S) \rightarrow \mu^{+} \mu^{-}$decays in data to calculate the efficiency through the tag-and-probe technique. The efficiency is factorised into three individual components,

$$
\epsilon_{\text {trig }}=\epsilon_{\mathrm{RoI}}\left(p_{T 1}^{\mu}, q_{1} \cdot \eta_{1}^{\mu}\right) \cdot \epsilon_{\mathrm{RoI}}\left(p_{T 2}^{\mu}, q_{2} \cdot \eta_{2}^{\mu}\right) \cdot c_{\mu^{+} \mu^{-}}\left(\Delta R,\left|y\left(\mu^{+} \mu^{-}\right)\right|\right)
$$

where $\epsilon_{\text {RoI }}$ is the efficiency with which the trigger system can identify a muon with transverse momentum $p_{T}^{\mu}$ and charge-signed pseudorapidity $q \cdot \eta^{\mu}$ as a muon Region of Interest (RoI). The single RoI efficiency $\epsilon_{\mathrm{RoI}}$ is binned in charge-signed pseudorapidity to account for a charge asymmetry in the muon identification efficiency caused by the magnetic field produced by the ATLAS toroid magnets.

The $c_{\mu^{+} \mu^{-}}$term accounts for the inefficiency associated with the "di-muon" aspects of the trigger algorithm and is parameterised as a function of the absolute rapidity of the di-muon system, $\left|y\left(\mu^{+} \mu^{-}\right)\right|$, and the spatial separation between the $\mu^{+}$and $\mu^{-}, \Delta R=\sqrt{(\Delta \phi)^{2}+(\Delta \eta)^{2}}$. The dominant "di-muon" effect is caused by the finite size of the muon RoI at L1 due to the limited granularity of the available information. Two muons which are spatially close (i.e $\Delta \eta$ and/or $\Delta \phi$ between the two muons is small) will tend to be identified as a single RoI as the angle between them decreases. Since the EF_2mu4(T)_Jpsimumu algorithm requires that two independent muon RoIs be identified for the event to be passed to the HLT, the trigger efficiency reduces as the angle between the two muons becomes smaller. The $\Delta R$ dependence of the $c_{\mu^{+} \mu^{-}}$term accounts for this effect in addition to further losses 
caused by di-muon vertex fit quality cuts applied in the HLT. The di-muon rapidity dependence of the $c_{\mu^{+} \mu^{-}}$correction accounts for the varying efficiency of the di-muon vertex fit quality requirement across different regions of the ID.

The $\epsilon_{\mathrm{RoI}}$ efficiency and the $\Delta R$ dependence of $c_{\mu^{+} \mu^{-}}$are both extracted from a sample of $J / \psi \rightarrow \mu^{+} \mu^{-}$and $\Upsilon(n S) \rightarrow \mu^{+} \mu^{-}$decays in data, selected by a single muon trigger with an $18 \mathrm{GeV}$ transverse momentum threshold. The muons which fire the single muon trigger are denoted the "tag" muons. The muons which are not matched to the single muon trigger object, denoted the "probe" muons, provide an unbiased sample of muons that can be used to determine $\epsilon_{\mathrm{RoI}}$ and measure the shape of $c_{\mu^{+} \mu^{-}}$as a function of $\Delta R$ (no trigger requirements are applied to the "probe" muons). The plateau efficiency of $c_{\mu^{+} \mu^{-}}$is determined by a sample of $J / \psi \rightarrow \mu^{+} \mu^{-}$ decays selected by a special calibration trigger that is identical to the nominal trigger but which does not apply any opposite charge, vertex quality or invariant mass cuts. The method used to determine the overall trigger efficiency is described in detail in [87].

The efficiencies of the EF_2mu4_Jpsimumu and EF_2mu4T_Jpsimumu triggers used to collect each half of the total dataset differ slightly due to the tighter selection required by the EF_2mu4T_Jpsimumu trigger. An independent efficiency correction is prepared for each trigger using the same method. The efficiency term $\epsilon_{\text {RoI }}$ for the EF_2mu4_Jpsimumu trigger is shown in Figure 7.7 and the $c_{\mu^{+} \mu^{-}}$correction for the EF_2mu4_Jpsimumu trigger is shown in Figure 7.8. 


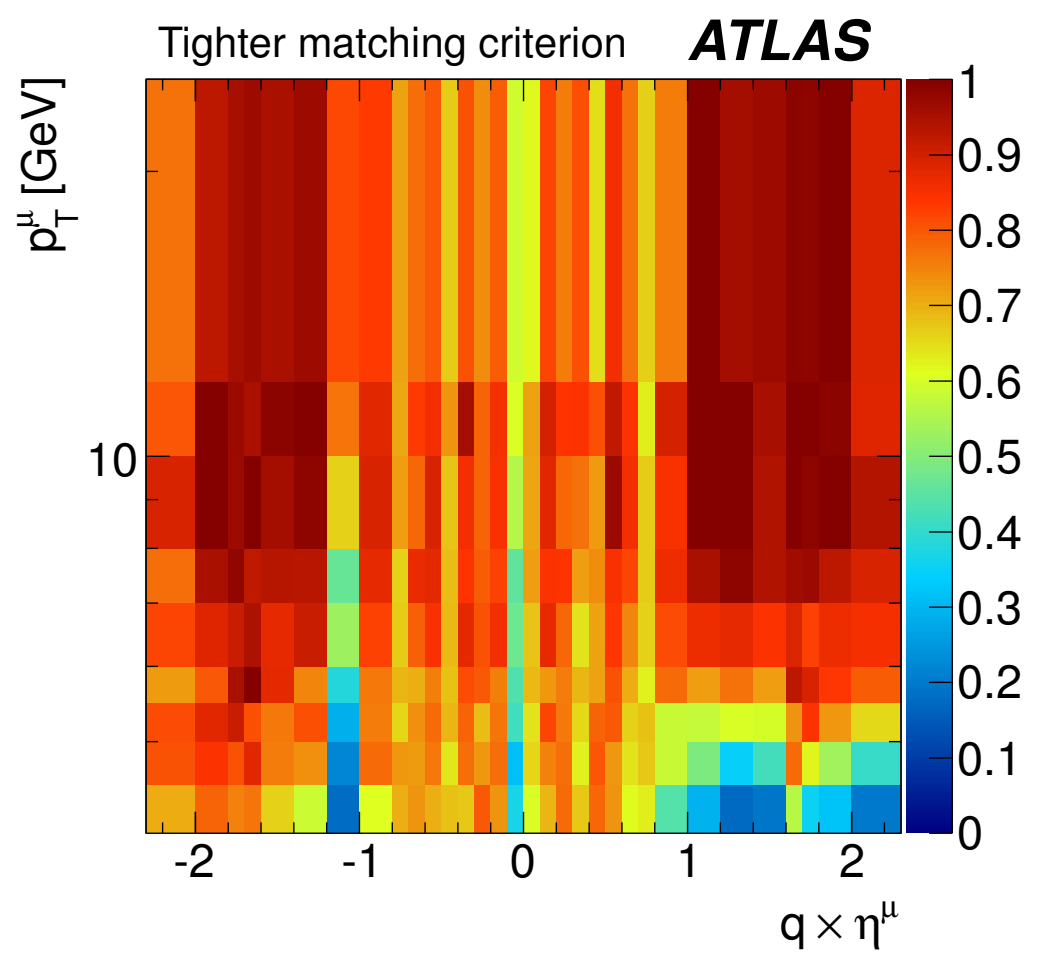

Figure 7.7: The single muon RoI efficiency component of the EF_2mu4 trigger efficiency, $\epsilon_{\text {RoI }}$. Figures from Ref. [87]. The "tighter matching criterion" simply refers to the $\Delta R<0.01$ matching requirement between the muon reconstructed in the trigger and offline reconstructed muon. 

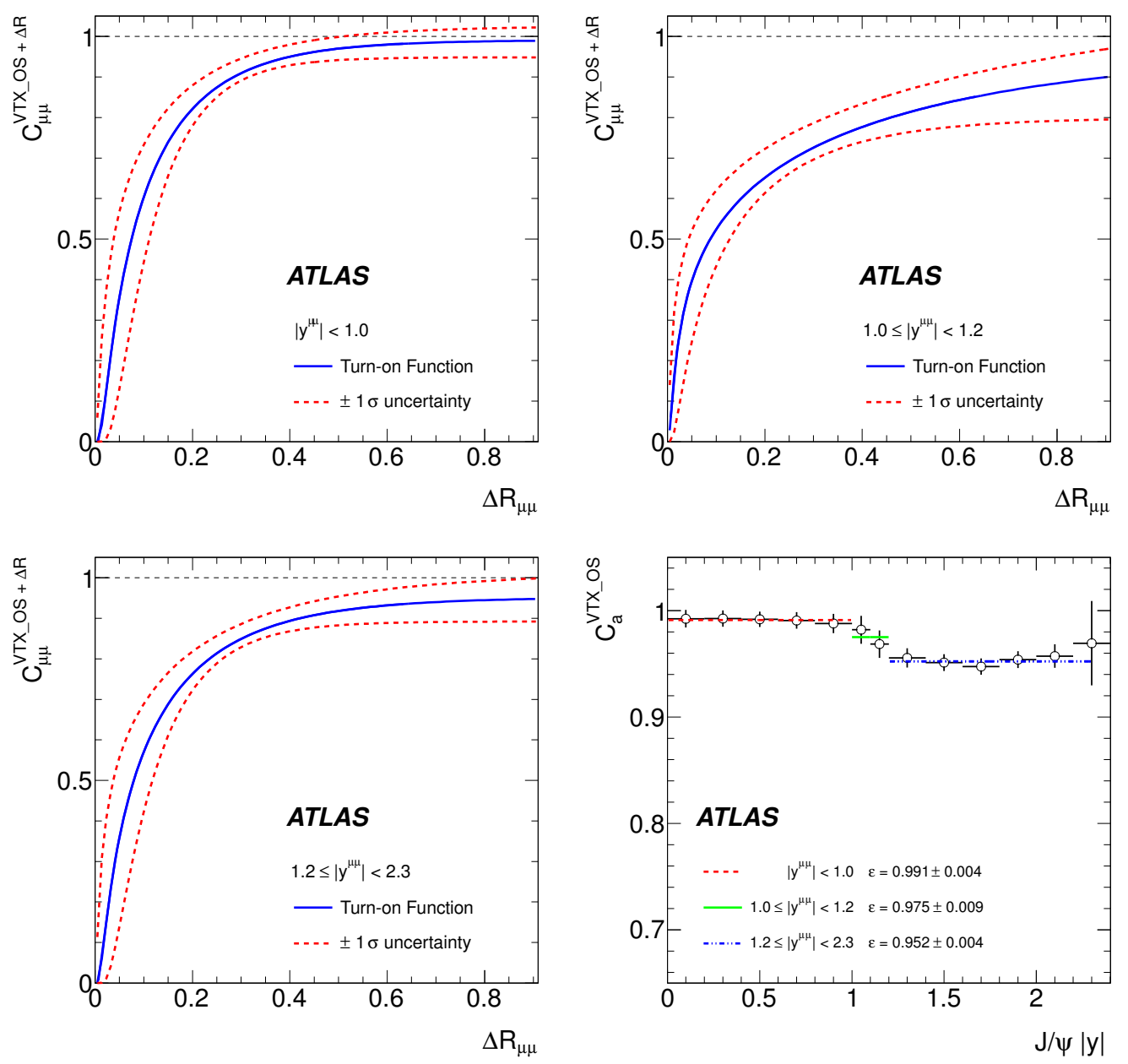

Figure 7.8: The di-muon correction to the EF_2mu4_Jpsimumu trigger efficiency, $c_{\mu^{+} \mu^{-}}$. The upper plots and the lower left plots show the di-muon correction term $c_{\mu^{+} \mu^{-}}$of $\epsilon_{\text {trig }}$, for different $\left|y^{J / \psi}\right|$ regions, as a function of $\Delta R$ measured between the two muons within the di-muon pair. The lower right plot shows the plateau values (at asymptotically large $\Delta R$ ) of $c_{\mu^{+} \mu^{-}}$as a function of the absolute rapidity of the di-muon system. Figures taken from Ref. [87]. The $\pm 1 \sigma$ systematic bands are used to estimate the systematic uncertainty on the measured $\chi_{c J}$ cross sections due to the parameterisation of the $c_{\mu^{+} \mu^{-}}$correction (this is discussed in detail in Section 7.8). The $y$ axis of the upper and lower left plots is identical to $c_{\mu^{+} \mu^{-}}$while the $y$ axis of the lower right plot represents the value that $c_{\mu^{+} \mu^{-}}$approaches asymptotically at large $\Delta R$. 


\subsection{3 $J / \psi \rightarrow \mu^{+} \mu^{-}$Reconstruction Efficiency}

The total efficiency with which $J / \psi \rightarrow \mu^{+} \mu^{-}$decays are reconstructed is factorised into several components,

$$
\epsilon_{\mathrm{di}-\mathrm{muon}}=\epsilon_{\mu}\left(p_{T 1}^{\mu}, q_{1} \cdot \eta_{1}^{\mu}\right) \cdot \epsilon_{\mu}\left(p_{T 2}^{\mu}, q_{2} \cdot \eta_{2}^{\mu}\right) \cdot \epsilon_{\mathrm{track}}^{2} \cdot \epsilon_{\mathrm{mass}}
$$

where $\epsilon_{\mu}$ is the muon identification efficiency, $\epsilon_{\text {track }}$ is the track reconstruction efficiency for muons, $\epsilon_{\text {mass }}$ is the efficiency of the $J / \psi \rightarrow \mu^{+} \mu^{-}$di-muon invariant mass selection $\left(2.95<m\left(\mu^{+} \mu^{-}\right)<3.25 \mathrm{GeV}\right)$. The quantities $p_{T}^{\mu}$ and $q \cdot \eta^{\mu}$ are the transverse momentum and charge-signed pseudorapidity of each muon. The muon track reconstruction efficiency, determined from simulation, reaches a plateau of

$\epsilon_{\text {track }}=0.99 \pm 0.01$ for genuine muons with $p_{T}^{\mu}>4 \mathrm{GeV}$ and $\left|\eta^{\mu}\right|<2.3$. This value has no significant dependence on the kinematics of the muons within this fiducial region [87]. The efficiency of the di-muon invariant mass selection $\epsilon_{\text {mass }}$ is determined to be $\epsilon_{\text {mass }}=0.990 \pm 0.005$ from a fit performed to the $J / \psi$ invariant mass peak, which accounts for the low mass tail of the di-muon resolution function caused by final state radiation (FSR). The muon identification efficiency, $\epsilon_{\mu}$, is measured using the tag-and-probe method from a sample of $J / \psi \rightarrow \mu^{+} \mu^{-}$decays in data. This measurement was prepared for use in the ATLAS study of $\Upsilon(n S)$ production, which was performed with the same di-muon selection and a subset of the data sample used in this analysis [87].

The muon identification efficiency is parametrised as an efficiency map binned in both muon transverse momentum $p_{T}^{\mu}$ and charge-signed pseudorapidity $q \cdot \eta^{\mu}$ as shown in Figure 7.9. As in the di-muon trigger efficiency correction, the muon identification efficiency is binned in charge-signed pseudorapidity to account for a charge asymmetry in the muon identification efficiency caused by the magnetic field produced by the ATLAS toroid magnets. In general, the efficiency approaches a plateau of around $98 \%$ for muons with transverse momentum in excess of $8 \mathrm{GeV}$. 
The efficiency map exhibits several localised regions of lower efficiency. The regions $q \cdot \eta^{\mu} \approx 0$ and $1.1<\left|q \cdot \eta^{\mu}\right|<1.3$ both represent regions of limited muon chamber coverage due to the join between the two halves of the barrel section of the MS and the transition region between the barrel and endcap regions of the MS, respectively.

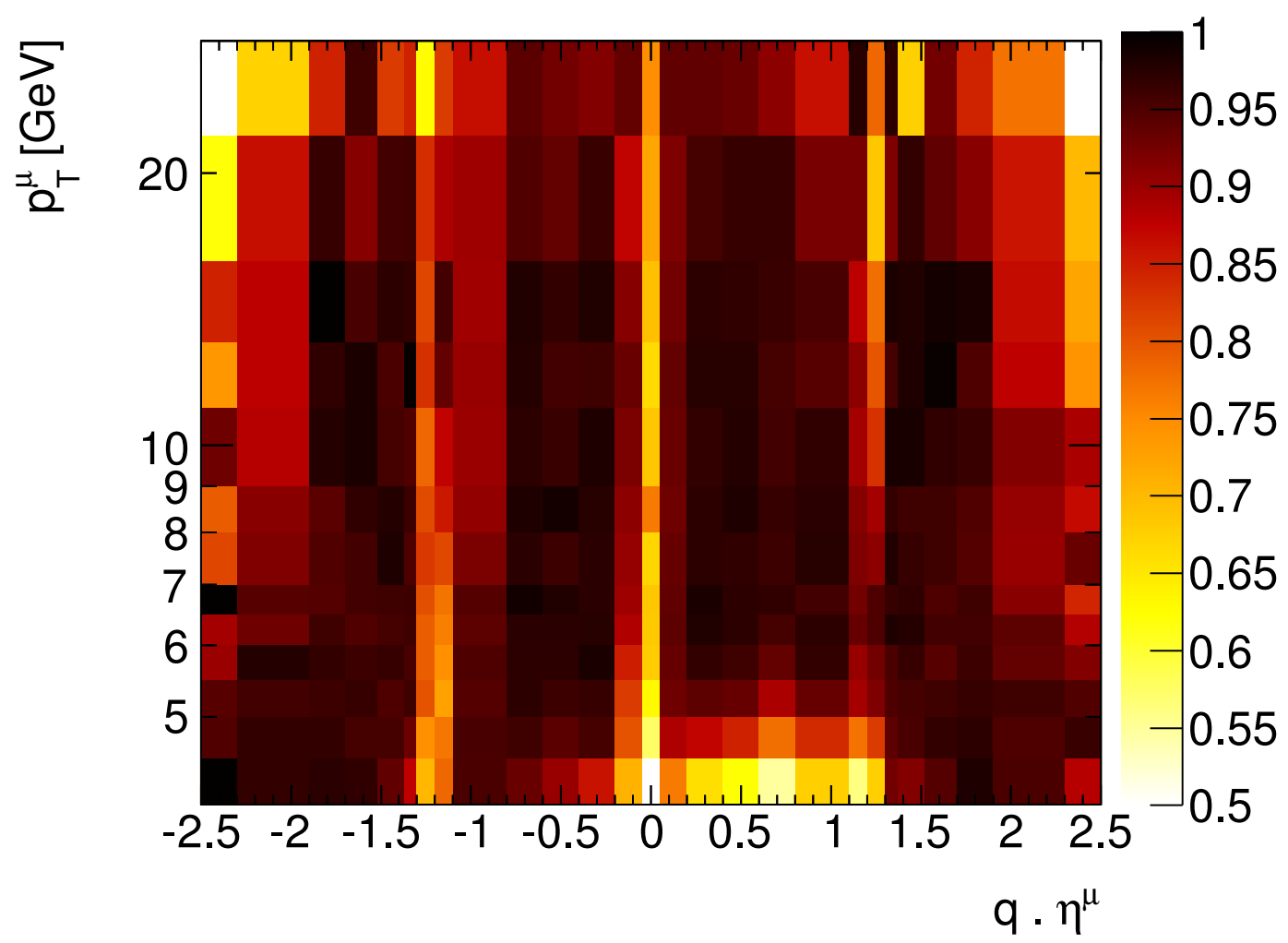

Figure 7.9: The muon identification efficiency as a function of reconstructed muon transverse momentum $p_{T}^{\mu}$ and charge-signed pseudorapidity $q \cdot \eta^{\mu}$. The efficiency map is determined using the tag-and-probe method from a sample of $J / \psi \rightarrow \mu^{+} \mu^{-}$ decays in data [87]. 


\subsubsection{Converted Photon Reconstruction Efficiency}

The converted photon reconstruction efficiency is estimated from the MC simulation samples described in Section 7.3. The total efficiency to reconstruct a photon as a conversion, $\epsilon_{\gamma}$, is factorised into two components,

$$
\epsilon_{\gamma}=P_{\text {conv }}\left(\eta^{\gamma}\right) \cdot \epsilon_{\text {conv }}\left(\left|\eta^{\gamma}\right|, p_{T}^{\gamma}\right)
$$

where $P_{\text {conv }}$ is the conversion probability and $\epsilon_{\text {conv }}$ is the conversion reconstruction efficiency. The conversion probability, $P_{\text {conv }}$, is essentially a function of the material distribution in the ID. The material distribution varies significantly as a function of $\eta$ (see Figures 5.8 and 5.9). To account for these variations, the conversion probability is calculated in bins of $\eta^{\gamma}$ from the ratio of the number of photons that convert within the region $40<r<150 \mathrm{~mm}$ to the total number of generated photons within the fiducial region $\left(p_{T}^{\gamma}>1.5 \mathrm{GeV}\right)$. The conversion probability is shown in Figure 7.10. No significant energy or transverse momentum dependence is observed for photons within the fiducial region, as expected from Figure 5.6 which shows that the photon conversion cross section saturates for photons with energies above $1 \mathrm{GeV}$.

The conversion reconstruction efficiency is calculated in bins of $p_{T}^{\gamma}$ and $\left|\eta^{\gamma}\right|$ from the ratio

$$
\epsilon_{\mathrm{conv}}=\frac{N_{\mathrm{reco}}^{\gamma}}{N_{\mathrm{gen}}^{\gamma}}
$$

where $N_{\text {reco }}^{\gamma}$ is the number of reconstructed converted photons and $N_{\text {gen }}^{\gamma}$ is the number of generated converted photons. To ensure that the simulated $\chi_{c}$ events are representative of those observed in the data, the distributions in the simulated events of the average number of $p p$ collisions in each bunch crossing and of the position in $z$ of the primary $p p$ interaction in the simulated events are re-weighted to follow the distributions observed in data.

When this efficiency is calculated for each reconstructed $\chi_{c J}$ candidate, only the 


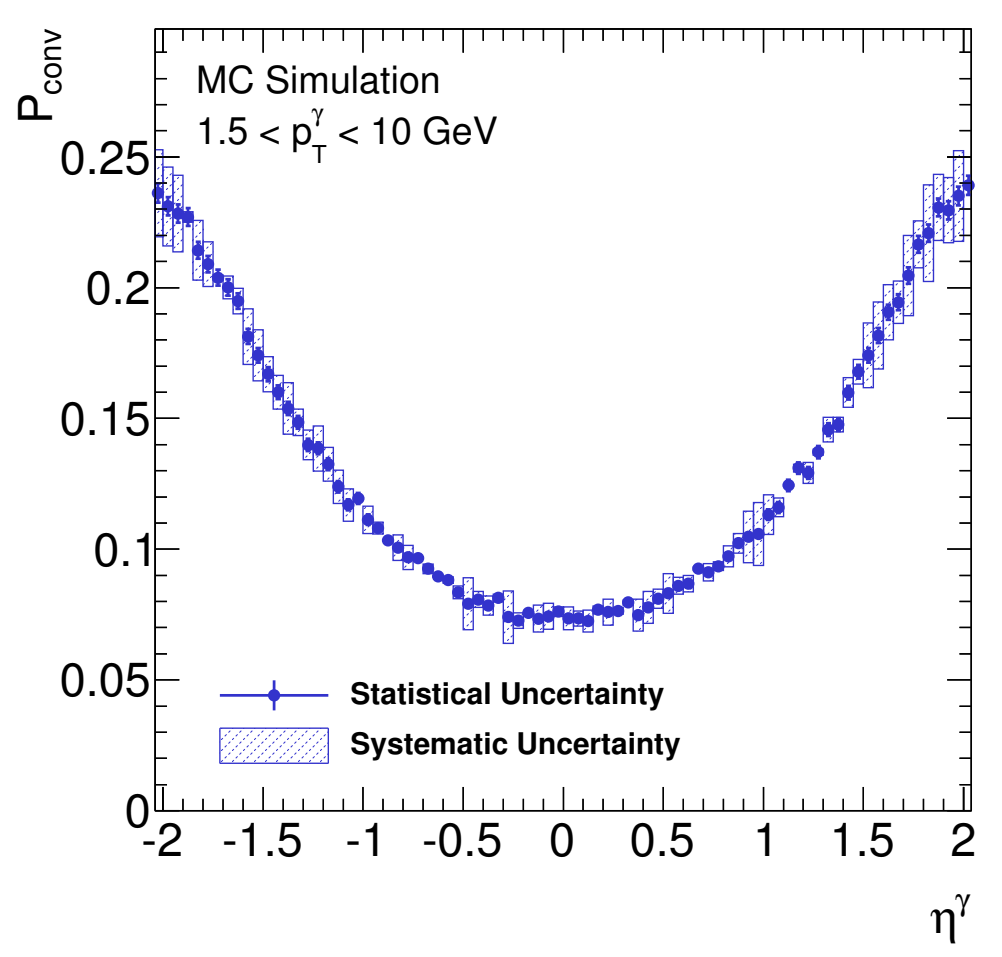

Figure 7.10: The conversion probability, derived from MC simulation, for photons with $p_{T}^{\gamma}>1.5 \mathrm{GeV}$ as a function of $\eta^{\gamma}$. The method used to estimate of the systematic uncertainty is discussed in Section 7.8.

reconstructed values of $p_{T}^{\gamma}$ and $\eta^{\gamma}$ are available. The experimental resolution in $\eta^{\gamma}$ is symmetric and very narrow and its use in calculating the per-candidate conversion probability and conversion reconstruction efficiency presents no problem. However, as shown in Figure 7.11, the experimental resolution in $p_{T}^{\gamma}$ is asymmetric and has a tail caused by electron energy losses in the ID. This presents a problem since, on average, the measured value of $p_{T}^{\gamma}$ will be less than the true value. This correction is often treated by building the ratio in Equation 7.8, by binning $N_{\text {reco }}^{\gamma}$ in the measured value of $p_{T}^{\gamma}$, and binning $N_{\mathrm{gen}}^{\gamma}$ in the true value of $p_{T}^{\gamma}$. This method is valid in the case that the generated $p_{T}^{\gamma}$ distribution does not vary significantly between the kinematic bins used to measure the cross section (i.e. $p_{T}^{J / \psi}$ and $p_{T}^{\chi_{c}}$ in this case), such that bin migrations in $p_{T}^{\gamma}$ are accurately taken into account. However, in the case of $\chi_{c J} \rightarrow J / \psi \gamma$ decays, the $p_{T}^{\gamma}$ distribution is strongly correlated to both $p_{T}^{J / \psi}$ and $p_{T}^{\chi_{c}}$ and varies significantly between bins. In this case, if one were to use the method 
described above, an individual efficiency would have to be calculated for each $p_{T}^{J / \psi}$ and $p_{T}^{\chi_{c}}$ bin to ensure bin migrations are accurately described. However, given the limited number of events within the MC samples, this is impractical and leads to large statistical uncertainties.

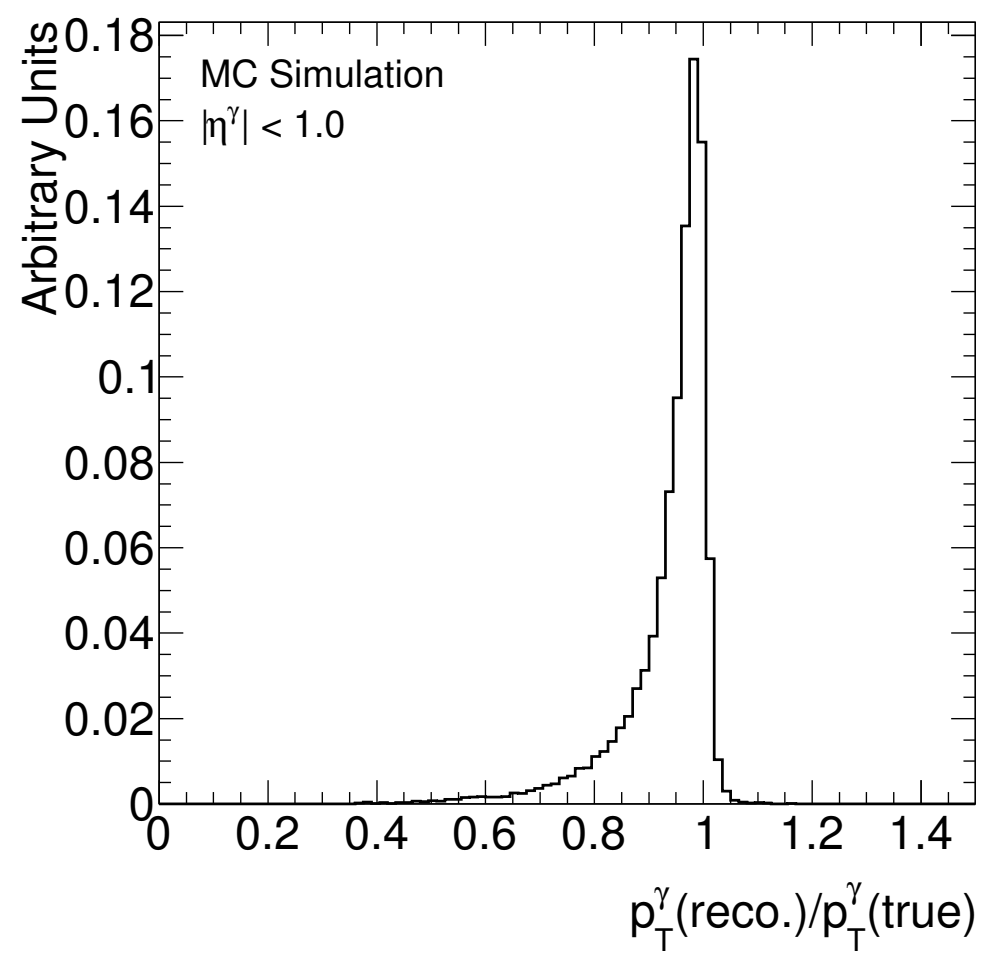

Figure 7.11: The normalised ratio of reconstructed $p_{T}^{\gamma}$ to true $p_{T}^{\gamma}$, determined from MC simulation.

An alternative approach can be used to form a single efficiency map that can be applied to calculate the per-candidate efficiency for $\chi_{c J}$ candidates in all of the measured $p_{T}^{\chi_{c}}$ and $p_{T}^{J / \psi}$ bins. In this approach, the ratio in Equation 7.8 is formed by binning $N_{\text {reco }}^{\gamma}$ in the measured value of $p_{T}^{\gamma}$, and binning $N_{\text {gen }}^{\gamma}$ in the true value of $p_{T}^{\gamma}$, but with a transformation applied. This ratio is calculated from converted photons generated within the fiducial region $p_{T}^{\gamma}>1.5 \mathrm{GeV}$, defined in terms of the true values of $p_{T}^{\gamma}$. Binning the numerator in the measured values of $p_{T}^{\gamma}$ and the denominator in the true values of $p_{T}^{\gamma}$ will lead to an efficiency map that can only be used to correct a data sample with a $p_{T}^{\gamma}$ distribution identical to that used to build the efficiency. To form a more "universal" efficiency, the true value of $p_{T}^{\gamma}$ is 
scaled by a multiplicative constant that is randomly sampled from an effective $p_{T}^{\gamma}$ resolution function. This resolution function represents the distribution of measured $p_{T}^{\gamma}$ as a function of true $p_{T}^{\gamma}$, with the fiducial requirement $p_{T}^{\gamma}>1.5 \mathrm{GeV}$ applied to the measured value of $p_{T}^{\gamma}$. The effective resolution function is shown in Figure 7.12. The scaled value of the true $p_{T}^{\gamma}$ is then used to bin the denominator of Equation 7.8.

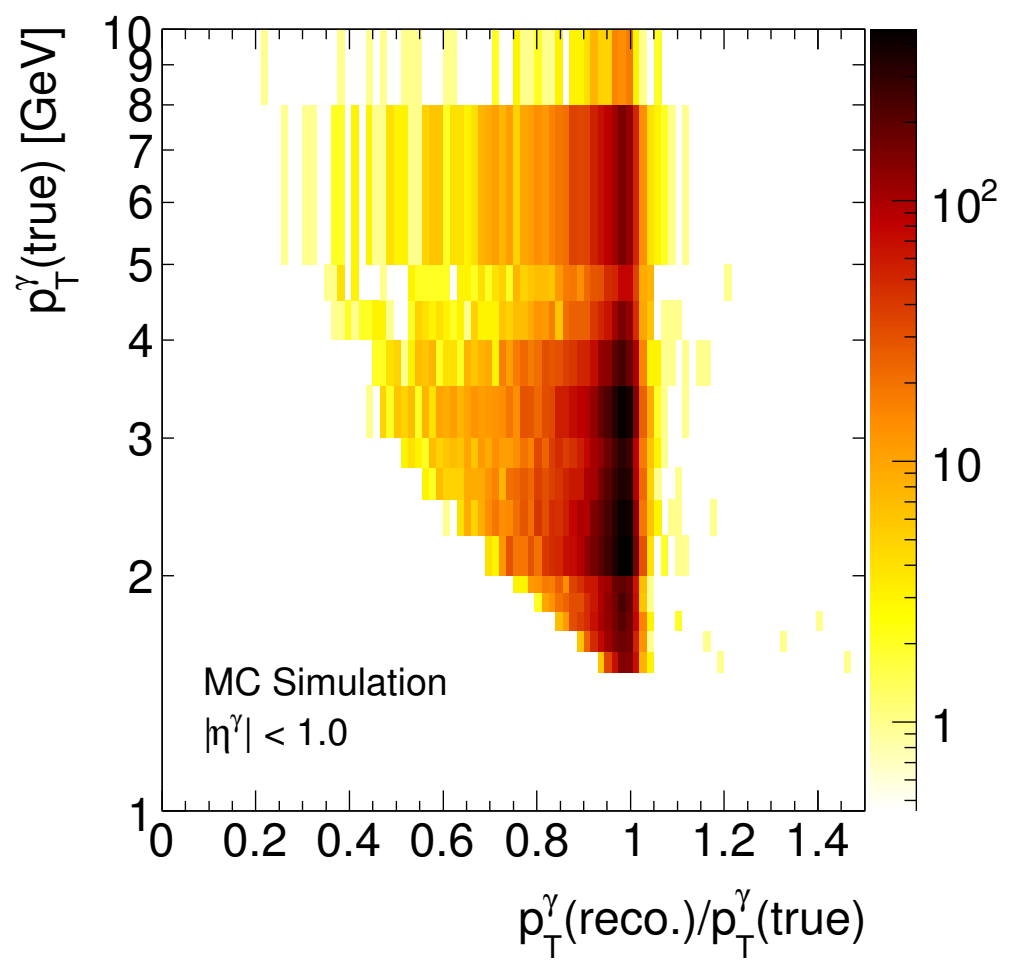

Figure 7.12: The effective experimental resolution in $p_{T}^{\gamma}$ (parametrised as the ratio of reconstructed $p_{T}^{\gamma}$ to true $p_{T}^{\gamma}$ ) as a function of the true value of $p_{T}^{\gamma}$. The fiducial cut $p_{T}^{\gamma}>1.5 \mathrm{GeV}$ is applied to the reconstructed value of $p_{T}^{\gamma}$. 
This method forms an efficiency map that can be used to correct $\chi_{c J}$ candidates within the fiducial region for the conversion reconstruction efficiency. The method described above is validated with toy simulations and is found to be accurate to within $\pm 5 \%$, within the fiducial region studied. This uncertainty is significantly lower than the statistical uncertainty that would be present in an ensemble of efficiency maps derived using the conventional method described earlier. Further validation of the method is performed with MC simulation samples, as is described in Section 7.5.5. The conversion reconstruction efficiency derived with this method is shown in Figures 7.13 and 7.14.

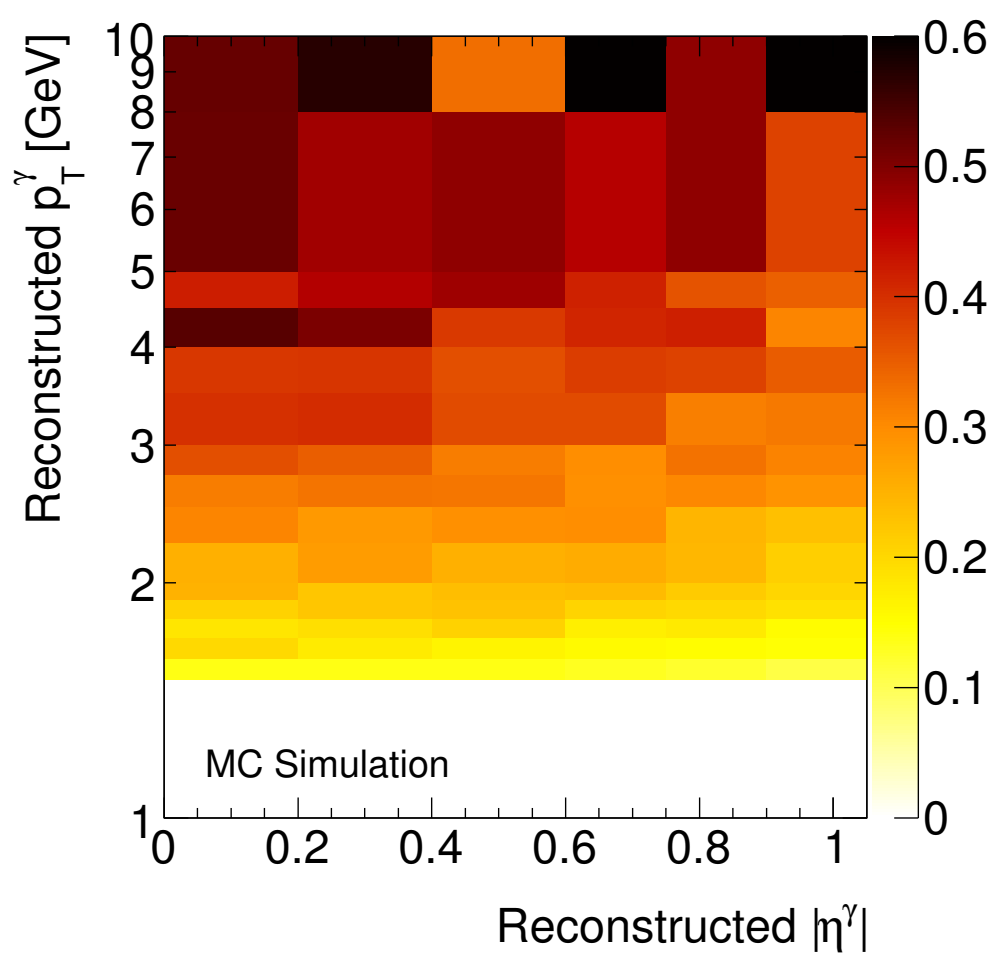

Figure 7.13: The conversion reconstruction efficiency calculated as a function of the measured values of $p_{T}^{\gamma}$ and $\left|\eta^{\gamma}\right|$. 

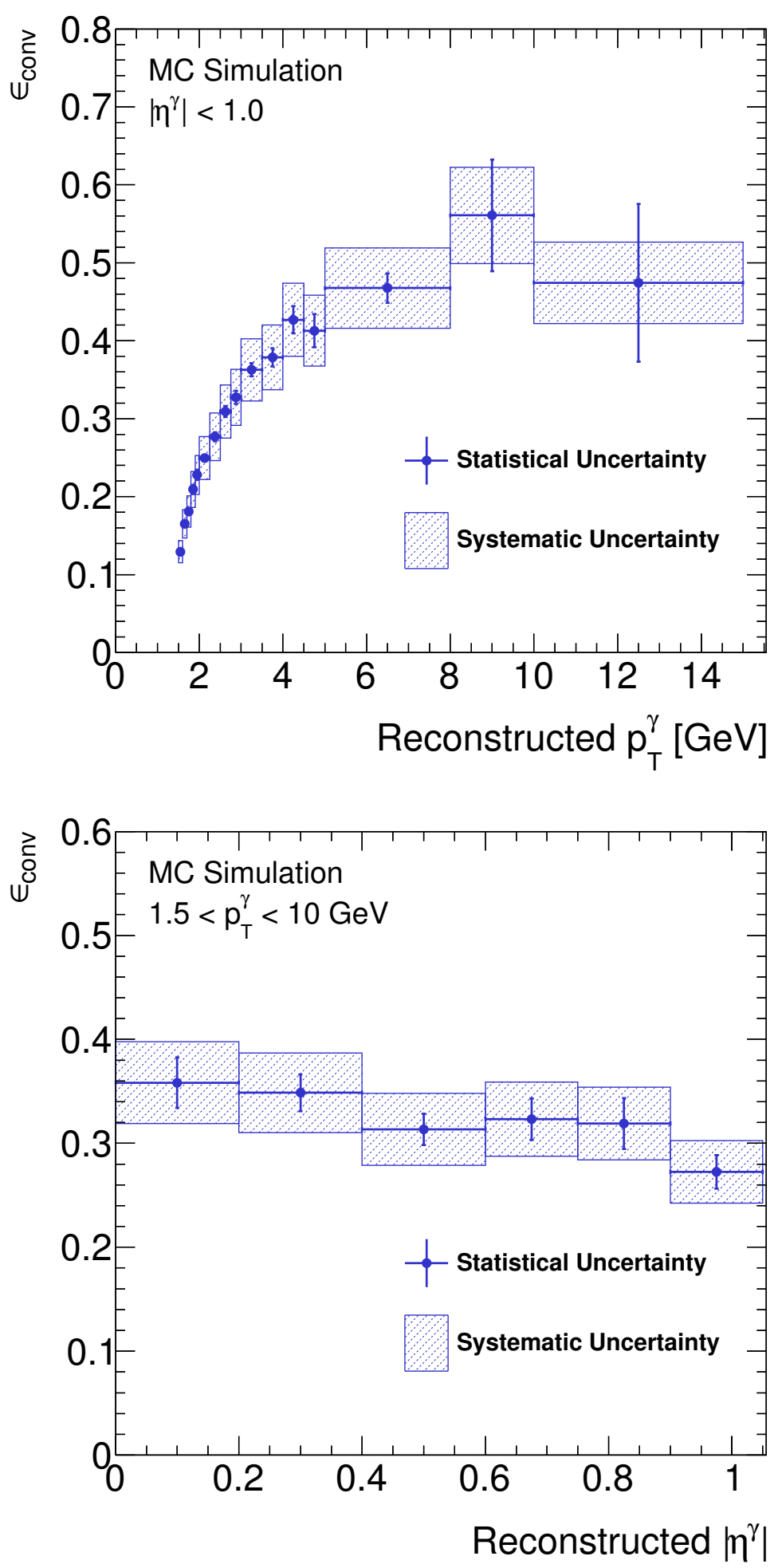

Figure 7.14: The conversion reconstruction efficiency calculated as a function of measured $p_{T}^{\gamma}$, for $\left|\eta^{\gamma}\right|<1.0$ (top) and as a function of measured $\left|\eta^{\gamma}\right|$, for $1.5<p_{T}^{\gamma} \mid<$ $10 \mathrm{GeV}$ (bottom). The estimate of the systematic uncertainty shown in both figures is discussed in Section 7.8. 


\subsubsection{Validation of Conversion Reconstruction Efficiency}

The accuracy of the procedure described in Section 7.5.4 is verified with a number of tests using the simulated $\chi_{c}$ event samples described in Section 7.3. $\chi_{c J} \rightarrow J / \psi \gamma$ decays generated in the fiducial region are reconstructed and weighted to account for conversion reconstruction efficiency. The efficiency-corrected yields of reconstructed $\chi_{c J} \rightarrow J / \psi \gamma$ decays are then compared to the true number of generated decays. To quantify the accuracy of the parametrisation of the conversion reconstruction efficiency, this test is performed in the same bins of $p_{T}^{\chi_{c}}$ and $p_{T}^{J / \psi}$ as used in the analysis. Figures 7.15 and 7.16 show the efficiency-corrected reconstructed yields divided by the true yields in bins of $p_{T}^{\chi_{c}}$ and $p_{T}^{J / \psi}$, respectively. This ratio is consistent

with unity across all $p_{T}^{\chi_{c}}$ and $p_{T}^{J / \psi}$ bins, suggesting that the parametrisation of the conversion reconstruction efficiency is accurate to within the statistical uncertainty. The systematic uncertainty on the method $( \pm 5 \%)$ derived from toy simulations is also shown. 

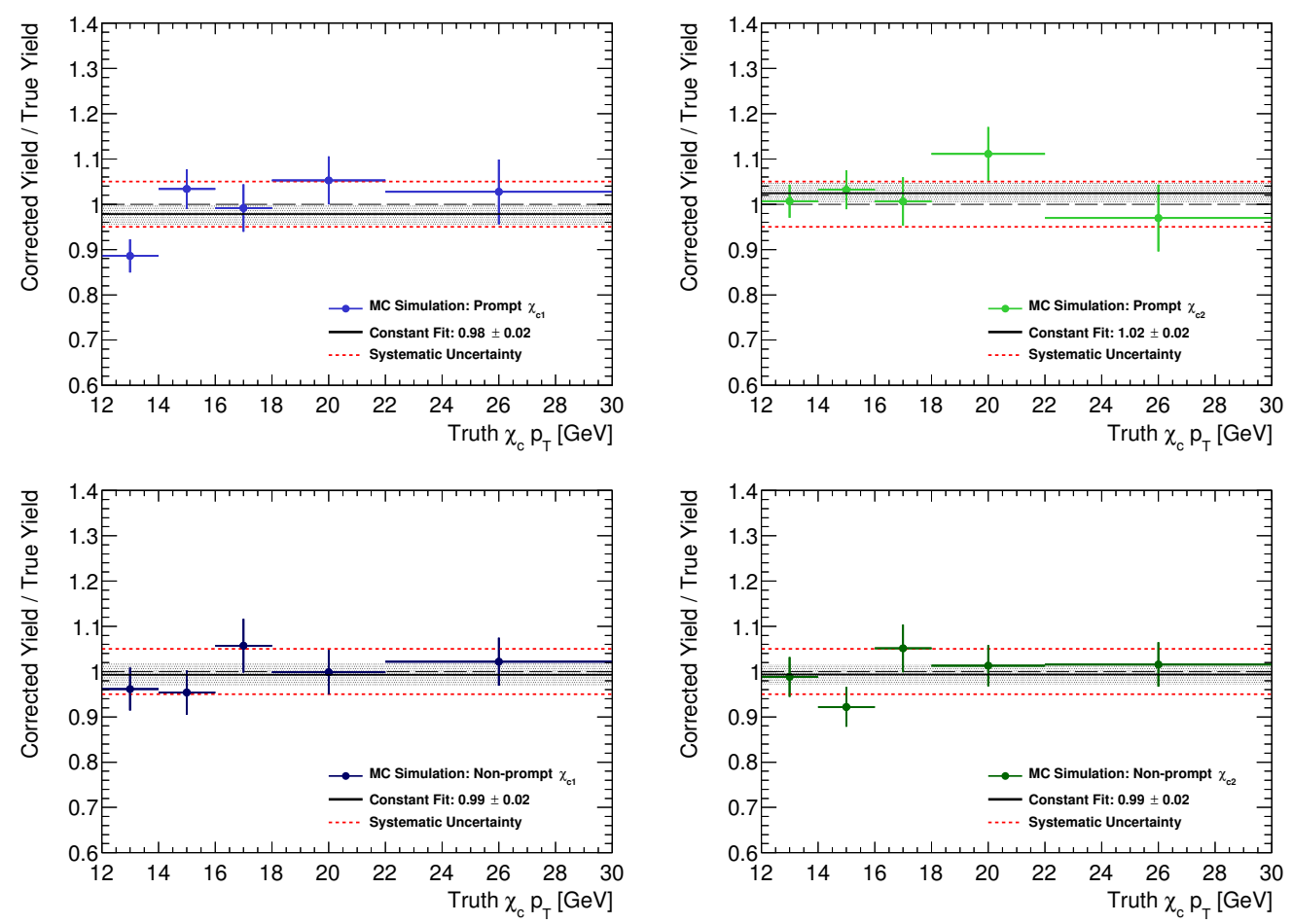

Figure 7.15: The efficiency corrected number of reconstructed simulated $\chi_{c J} \rightarrow$ $J / \psi \gamma$ decays divided by the true number of $\chi_{c J} \rightarrow J / \psi \gamma$ decays, measured in bins of $p_{T}^{\chi_{c}}$. The systematic uncertainty on the method $( \pm 5 \%)$ derived from toy simulations is also shown as the dashed red line. 

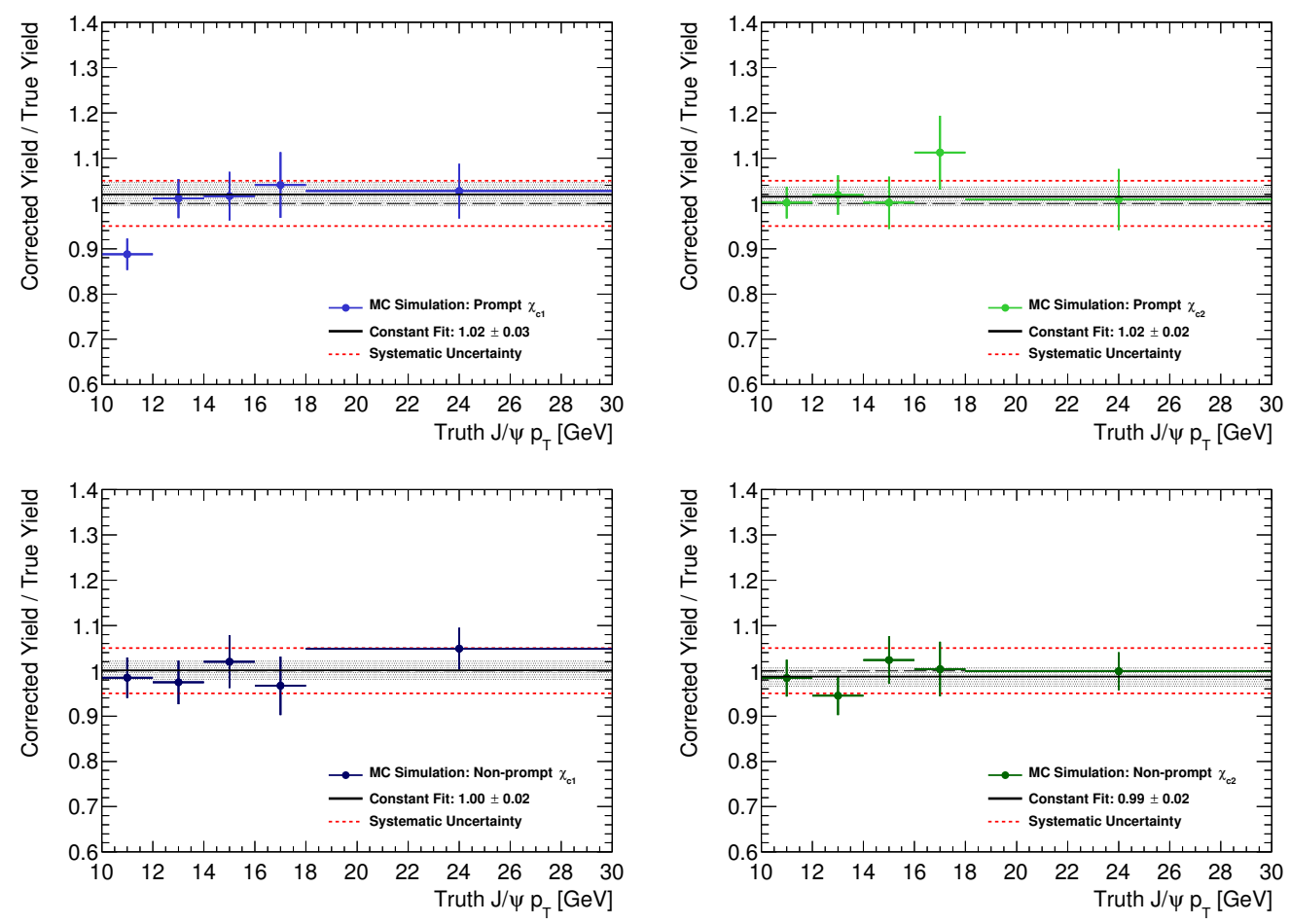

Figure 7.16: The efficiency corrected number of reconstructed simulated $\chi_{c J} \rightarrow$ $J / \psi \gamma$ decays divided by the true number of $\chi_{c J} \rightarrow J / \psi \gamma$ decays, measured in bins of $p_{T}^{J / \psi}$. The systematic uncertainty on the method $( \pm 5 \%)$ derived from toy simulations is also shown as the dashed red line. 


\subsection{Measurement of corrected $\chi_{c J}$ yields}

The efficiency and acceptance-corrected prompt and non-prompt $\chi_{c}$ yields are extracted with a weighted, simultaneous, unbinned maximum likelihood fit to the mass difference, $\Delta m=m\left(\mu^{+} \mu^{-} \gamma\right)-m\left(\mu^{+} \mu^{-}\right)$, and pseudo-proper decay time, $\tau$,

distributions. Fits are performed in bins of both $p_{T}^{\chi_{c}}$ and $p_{T}^{J / \psi}$. A simultaneous unbinned fit to the $\Delta m$ and $\tau$ distributions allows the corrected prompt and nonprompt $\chi_{c 1}$ and $\chi_{c 2}$ yields to be extracted directly in a single fit, as described in Section 5.6.2. This method avoids the potential biases associated with fitting the two distributions separately and has been used in many charmonium production measurements made at the LHC [76-79]. Fits are performed within the restricted region $0.2<\Delta m<0.7 \mathrm{GeV}$, corresponding to the $\chi_{c}$ signal region (including a sideband region for background normalisation), while no restriction on $\tau$ is applied.

The full probability density function (PDF) used to perform the fit takes the form,

$$
P(\Delta m, \tau, \delta \tau)=f_{\mathrm{sig}} \cdot P_{\mathrm{sig}}(\Delta m, \tau, \delta \tau)+\left(1-f_{\mathrm{sig}}\right) \cdot P_{\mathrm{bkgd}}(\Delta m, \tau, \delta \tau),
$$

where $f_{\text {sig }}$ is the fraction of $\chi_{c}$ signal events, $P_{\text {sig }}$ is the $\chi_{c}$ signal PDF and $P_{\mathrm{bkgd}}$ is the background PDF. The quantity $\delta \tau$ is the per-candidate uncertainty on the pseudo-proper decay time $\tau$ and is determined from the covariance matrix of the di-muon track vertex fit. The distributions of the pseudo-proper decay time uncertainty $\delta \tau$ within the signal-enriched region, $0.30<\Delta m<0.48 \mathrm{GeV}$, and within the background-dominated regions, $\Delta m<0.30 \mathrm{GeV}$ and $\Delta m>0.50 \mathrm{GeV}$, are observed to be consistent. As a consequence of this, the so-called "Punzi" terms [150] in the full PDF associated with the $\delta \tau$ distribution can be factorised. These factorised terms simply contribute a constant offset in the likelihood function and are removed 
from Equation 7.9 for simplicity. The $\chi_{c}$ signal and background PDFs, $P_{\text {sig }}$ and $P_{\mathrm{bkgd}}$, are each described by products of a PDF describing the $\Delta m$ projection of the fit (all denoted by $M$ ) and a PDF describing the $\tau$ projection of the fit (all denoted by $T)$.

\subsubsection{Modelling of the $\chi_{c J}$ signals}

The total $\chi_{c}$ signal PDF is given by,

$$
\begin{aligned}
P_{\text {sig }}(\Delta m, \tau, \delta \tau)= & f_{\text {sig }}^{\mathrm{P}} \cdot\left[f_{0}^{\mathrm{P}} \cdot M_{0}(\Delta m)+\left(1-f_{0}^{\mathrm{P}}\right) \cdot\left(f_{1}^{\mathrm{P}} \cdot M_{1}(\Delta m)\right.\right. \\
& \left.\left.+\left(1-f_{1}^{\mathrm{P}}\right) \cdot M_{2}(\Delta m)\right)\right] \cdot T_{\text {sig }}^{\mathrm{P}}(\tau, \delta \tau) \\
& +\left(1-f_{\mathrm{sig}}^{\mathrm{P}}\right) \cdot\left[f_{0}^{\mathrm{NP}} \cdot M_{0}(\Delta m)+\left(1-f_{0}^{\mathrm{NP}}\right) \cdot\left(f_{1}^{\mathrm{NP}} \cdot M_{1}(\Delta m)\right.\right. \\
& \left.\left.+\left(1-f_{1}^{\mathrm{NP}}\right) \cdot M_{2}(\Delta m)\right)\right] \cdot T_{\text {sig }}^{\mathrm{NP}}(\tau, \delta \tau),
\end{aligned}
$$

where $f_{\text {sig }}^{\mathrm{P}}$ is the fraction of signal candidates that are prompt $\chi_{c}$ signal candidates, $f_{0}^{(\mathrm{N}) \mathrm{P}}$ is the fraction of (non-)prompt signal candidates identified as $\chi_{c 0}$ and $f_{1}^{(\mathrm{N}) \mathrm{P}}$ is the fraction of (non-)prompt signal candidates identified as $\chi_{c 1}$ (excluding $\chi_{c 0}$ contributions). The PDFs $M_{J}(\Delta m)$ model the $\chi_{c J}$ signal peaks within the $\Delta m$ distribution and $T_{\mathrm{sig}}^{(\mathrm{N}) \mathrm{P}}(\tau, \delta \tau)$ are PDFs which describe the shape of the (non-)prompt $\chi_{c}$ signal contributions to the $\tau$ distribution.

The PDFs $M_{J}(\Delta m)$, describing the $\chi_{c 0}, \chi_{c 1}$ and $\chi_{c 2}$ signals in the $\Delta m$ distribution, are each modelled by Crystal Ball (CB) functions [151,152]. The CB PDF is characterised by a Gaussian core with a mean $\bar{m}_{J}$ and width $\sigma_{J}$, a low mass powerlaw tail described by a parameter $n$, and a final parameter, $\alpha$, which describes the transition between the core and the tail components of the PDF. The resolution in $\Delta m$ for $\chi_{c J}$ decays is studied with the simulated $\chi_{c}$ samples described in Section 7.3. Reconstructed $\chi_{c J} \rightarrow J / \psi \gamma$ decays satisfying the event selection described in Section 7.4 are matched to true decays by requiring that the angles between the 
reconstructed and true momenta of all three final state decay products $\left(\mu^{+}, \mu^{-}\right.$, $\gamma)$ each satisfy $\Delta R<0.1$. The reconstructed $\Delta m$ distributions for these simulated decays are then fitted with a single CB function, as shown in Figure 7.17. The prompt $\chi_{c J}$ simulation samples were produced with no natural decay width, while the non-prompt $\chi_{c J}$ simulation samples all include a natural width (with values consistent with the current world averages). The fits to these simulated samples are used to inform the modelling of the $\chi_{c}$ signal PDFs, as discussed below. No significant dependence of the CB parameters on $p_{T}^{J / \psi}$ or $p_{T}^{\chi_{c}}$ is observed in the simulated samples.

- $\bar{m}_{1,2}$ : The mean values for the $\chi_{c 1}$ and $\chi_{c 2}$ signals, $\bar{m}_{1,2}$ are fixed by the worldaverage values for the mass differences between these two states and the $J / \psi$ mass, $\Delta m_{J}^{\mathrm{PDG}}[3]$. These values are multiplied by a common multiplicative scale factor, $\lambda$, present to account for a small downward shift in the mean values of the observed peaks due to electron energy losses in the ID: $\bar{m}_{J}=\lambda \cdot \Delta m_{J}^{\mathrm{PDG}}$. The parameter $\lambda$ is freely determined by the fit procedure and typically takes values of around 0.98 .

- $\alpha$ and $n$ : The parameters $\alpha$ and $n$ are found to be consistent for both prompt and non-prompt $\chi_{c 1}$ and $\chi_{c 2}$, as shown in Figure 7.17. Motivated by these observations, the values of these parameters are fixed, when fitting to data, to the values $\alpha=0.6$ and $n=2.9$. Only the non-prompt samples are used to determine these values, as they include a simulation of the natural width of the $\chi_{c}$ states. This pair of parameters was chosen by first fixing the parameter $\alpha=0.6$ and repeating the fit with $n$ free. A value of $n=2.9$ (determined from the average of the two values determined from fits to the non-prompt $\chi_{c 1}$ and $\chi_{c 2}$ samples, once the parameter $\alpha=0.6$ has been fixed) was found to describe both the $\chi_{c 1}$ and $\chi_{c 2}$ signal shapes well.

- $\sigma_{1,2}$ : The natural widths of the $\chi_{c 1}$ and $\chi_{c 2}$ states $\left(\Gamma_{1}=0.86 \pm 0.05 \mathrm{MeV}\right.$ and 
$\left.\Gamma_{2}=1.97 \pm 0.11 \mathrm{MeV}[3]\right)$ are sufficiently lower than the experimental resolution $(\sigma \approx 10 \mathrm{MeV})$ that their effects on the $\chi_{c 1}$ and $\chi_{c 2}$ signals are not fitted explicitly (though their effect is included in the MC simulation from which the $\mathrm{CB}$ parameters are determined). The parameter $\sigma_{1}$ is freely determined by the fit, while the constraint $\sigma_{2}=k \cdot \sigma_{1}$, where $k$ is a constant, is imposed to reduce the overall number of free parameters. A value of $k=1.07$ is determined from fits to the MC simulation samples performed with the $\mathrm{CB}$ parameters $\alpha$ and $n$ fixed to the values $\alpha=0.6$ and $n=2.9$.

- Modelling of the $\chi_{c 0}$ signal: The PDF describing the $\chi_{c 0}$ signal is modelled independently of the $\chi_{c 1}$ and $\chi_{c 2}$ signals. The much larger natural width $\left(\Gamma_{1}=10.3 \pm 0.6 \mathrm{MeV}[3]\right)$ of the $J=0$ state requires that an alternative PDF be used to model this signal. An alternative PDF composed of the sum of a CB function and a Gaussian (with an independent width but with a mean identical to that of the CB function) is chosen, as shown in the top-right plot in Figure 7.17. All parameters of the CB function, the relative normalisation of the Gaussian component and its width are all fixed to the values shown in Figure 7.17.

The PDF describing the prompt $\chi_{c J}$ signal contribution to the $\tau$ distribution, $T_{\text {sig }}^{\mathrm{P}}(\tau, \delta \tau)$, is modelled with a Dirac delta function, $\delta(\tau)$, while the $\operatorname{PDF} T_{\text {sig }}^{\mathrm{NP}}(\tau, \delta \tau)$, describing the non-prompt $\chi_{c J}$ signal contribution to the $\tau$ distribution, is modelled by an exponential function $\exp \left(-\tau / \tau_{\text {sig }}\right)$. The parameter $\tau_{\text {sig }}$ is freely determined in the fit procedure. Both of these PDFs are convolved with the function $R\left(\tau^{\prime}-\tau, \delta \tau\right)$ describing the experimental resolution in $\tau$. The function $R$ is represented by a Gaussian function with a mean of zero and a width given by $S \cdot \delta \tau$, where $S$ is a scale factor and $\delta \tau$ is the per-candidate uncertainty on the pseudo-proper decay time $\tau$. The parameter $S$ is freely determined by the fit procedure and typically takes values of approximately 1.02 . 

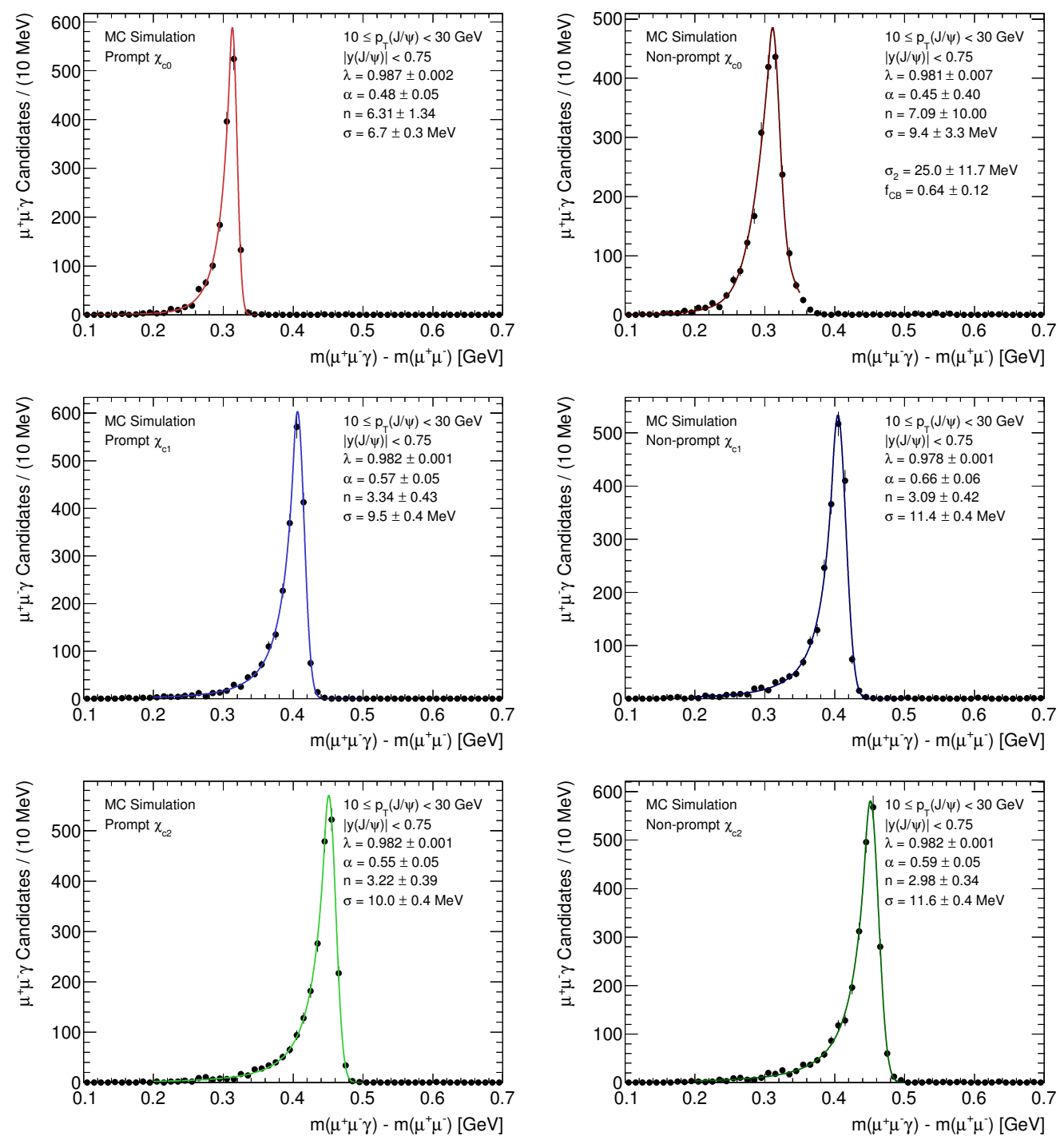

Figure 7.17: $\Delta m$ distributions for simulated prompt and non-prompt $\chi_{c J}$ events, each fitted with a Crystal Ball function. 


\subsubsection{Modelling of the background}

The background PDF, $P_{\mathrm{bkgd}}$, is given by,

$$
\begin{aligned}
P_{\mathrm{bkgd}}(\Delta m, \tau, \delta \tau)= & f_{\mathrm{bkgd}}^{\mathrm{P}} \cdot M_{\mathrm{bkgd}}^{\mathrm{P}}(\Delta m) \cdot T_{\mathrm{bkgd}}^{\mathrm{P}}(\tau, \delta \tau) \\
& +\left(1-f_{\mathrm{bkgd}}^{\mathrm{P}}\right) \cdot M_{\mathrm{bkgd}}^{\mathrm{NP}}(\Delta m) \cdot T_{\mathrm{bkgd}}^{\mathrm{NP}}(\tau, \delta \tau),
\end{aligned}
$$

where $f_{\mathrm{bkgd}}^{\mathrm{P}}$ is the fraction of background candidates that are promptly produced and the PDFs $M_{\mathrm{bkgd}}^{(\mathrm{N}) \mathrm{P}}$ and $T_{\mathrm{bkgd}}^{(\mathrm{N}) \mathrm{P}}$ describe the (non-)prompt background contributions to the $\Delta m$ and $\tau$ distributions, respectively. The PDF describing both the prompt and non-prompt background contributions to the $\Delta m$ distribution takes the form,

$$
M_{\mathrm{bkgd}}(\Delta m)=\operatorname{erf}\left(A \cdot\left(\Delta m-m_{0}\right)\right) \cdot \exp \left(B \cdot\left(\Delta m-m_{0}\right)\right)+C \cdot\left(\Delta m-m_{0}\right)^{2}
$$

where erf denotes the error function. All four parameters describing the shape of the distribution $\left(A, B, C\right.$ and $\left.m_{0}\right)$ are freely determined by the fit procedure. The analytical form of the $M_{\mathrm{bkgd}} \mathrm{PDF}$ is motivated by studies with the MC simulation and the shape of the $\Delta m$ distribution in data for $\mu^{+} \mu^{-} \gamma$ candidates with an invariant mass outside the $J / \psi$ peak. The prompt and non-prompt background PDFs, $M_{\mathrm{bkgd}}^{P}$ and $M_{\mathrm{bkgd}}^{N P}$, are each modelled with an independent set of the parameters $A, B$ and $C\left(m_{0}\right.$ is common) since the shape of the background in $\Delta m$ is observed to differ as a function of $\tau$ (i.e. significant additional contributions from $b \rightarrow J / \psi X$ decays contribute to the non-prompt $\Delta m$ background distribution).

The PDF describing the prompt background contribution to the $\tau$ distribution, $T_{\text {bkgd }}^{\mathrm{P}}$, is modelled with a delta function convolved with the resolution function $R$, as for the prompt $\chi_{c}$ signal PDF, $T_{\text {sig }}^{\mathrm{P}}$. The non-prompt background contribution to the $\tau$ distribution, $T_{\mathrm{bkgd}}^{\mathrm{NP}}$, is modelled by a sum of a single and a double-sided 
exponential function,

$$
\begin{aligned}
T_{\mathrm{bkgd}}^{\mathrm{NP}}(\tau, \delta \tau)= & {\left[\frac{g_{\mathrm{bkgd}}}{\tau_{\mathrm{bkgd}}} \cdot \exp \left(-\tau^{\prime} / \tau_{\mathrm{bkgd}}\right)\right.} \\
& \left.+\frac{\left(1-g_{\mathrm{bkgd}}\right)}{2 \tau_{\mathrm{sym}}} \cdot \exp \left(-\left|\tau^{\prime}\right| / \tau_{\mathrm{sym}}\right)\right] \otimes R\left(\tau^{\prime}-\tau, \delta \tau\right),
\end{aligned}
$$

where the parameter $g_{\mathrm{bkgd}}$ represents the relative mixture of the single and doublesided exponential functions and $\tau_{\mathrm{bkgd}}$ and $\tau_{\mathrm{sym}}$ are slope parameters for the single and a double-sided exponential functions, respectively. The parameters $\tau_{\text {bkgd }}$ and $g_{\mathrm{bkgd}}$ are freely determined by the fit procedure. No significant variation in $\tau_{\text {sym }}$ is observed as a function of $p_{T}^{\chi_{c}}$ or $p_{T}^{J / \psi}$ and its value is fixed to a value determined in a 1-dimensional fit to the $\tau$ distribution of the inclusive data sample, $\tau_{\mathrm{sym}}=0.25$.

The validation of the full fit procedure is performed with fits to toy MC simulated data and is described in detail in Appendix B. Since the acceptance corrections for prompt and non-prompt $\chi_{c 1}$ and $\chi_{c 2}$ differ, the fit procedure is performed four times for each $p_{T}^{J / \psi}$ and $p_{T}^{\chi_{c}}$ bin, once for each different acceptance correction (i.e. prompt $\chi_{c 1}$, non-prompt $\chi_{c 1}$, prompt $\chi_{c 2}$ and non-prompt $\left.\chi_{c 2}\right)$. In each fit, only the $\chi_{c J}$ yield corresponding to the relevant acceptance correction (e.g. the prompt $\chi_{c 1}$ yield in a fit to data weighted with the prompt $\chi_{c 1}$ acceptance correction) is saved while the other three yields are discarded.

The fit result for the entire data sample, $10 \leq p_{T}^{J / \psi}<30 \mathrm{GeV}$, is shown in Figure 7.18. Examples of fit results for individual $p_{T}^{J / \psi}$ and $p_{T}^{\chi_{c}}$ bins are shown in Figures 7.19 and 7.20, respectively. 

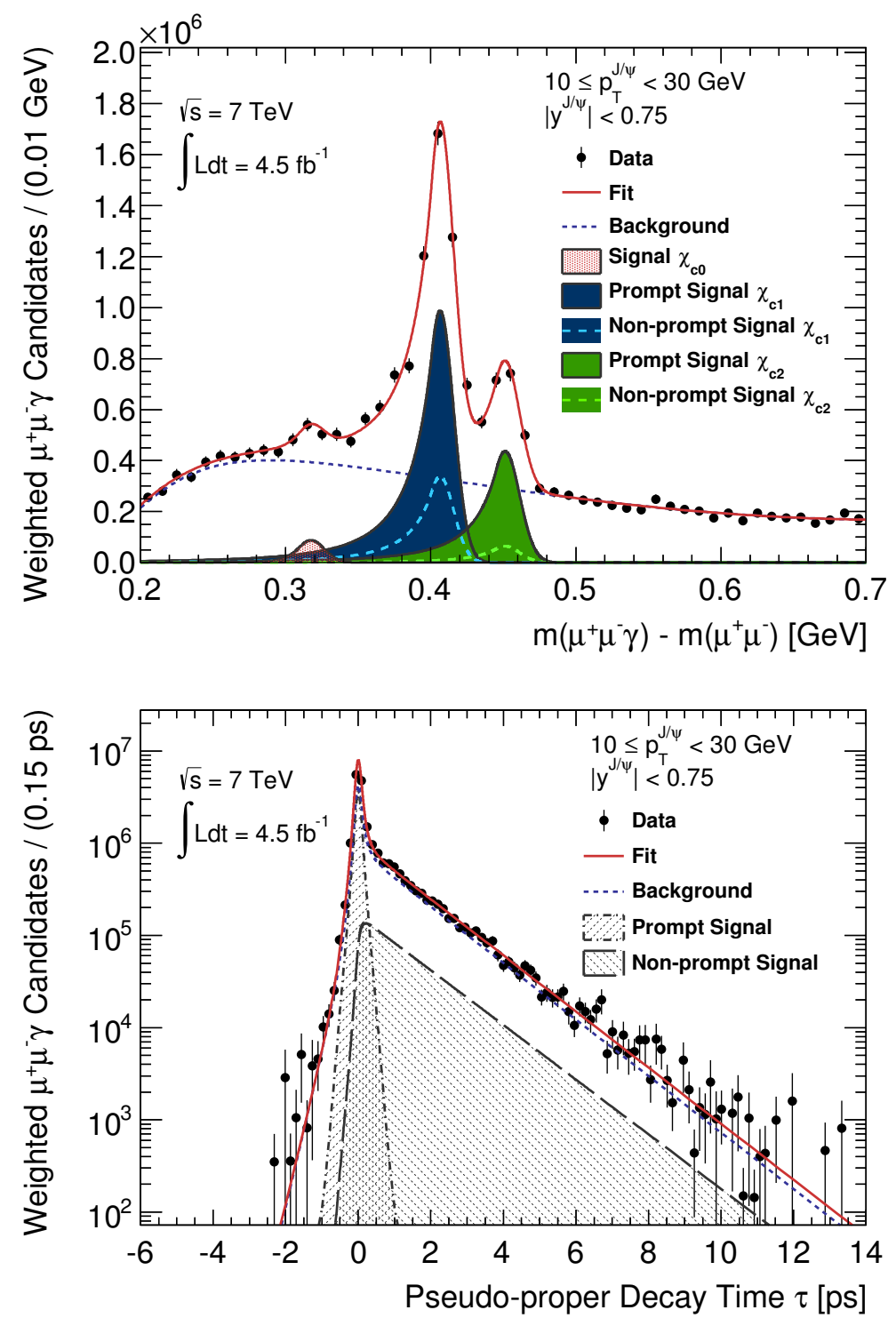

Figure 7.18: The $\Delta m$ and $\tau$ projections of a simultaneous unbinned maximum likelihood fit performed to the inclusive sample of reconstructed $\chi_{c J}$ decays $(10 \leq$ $\left.p_{T}^{J / \psi}<30 \mathrm{GeV}\right)$, weighted with the prompt $\chi_{c 1}$ acceptance correction. 

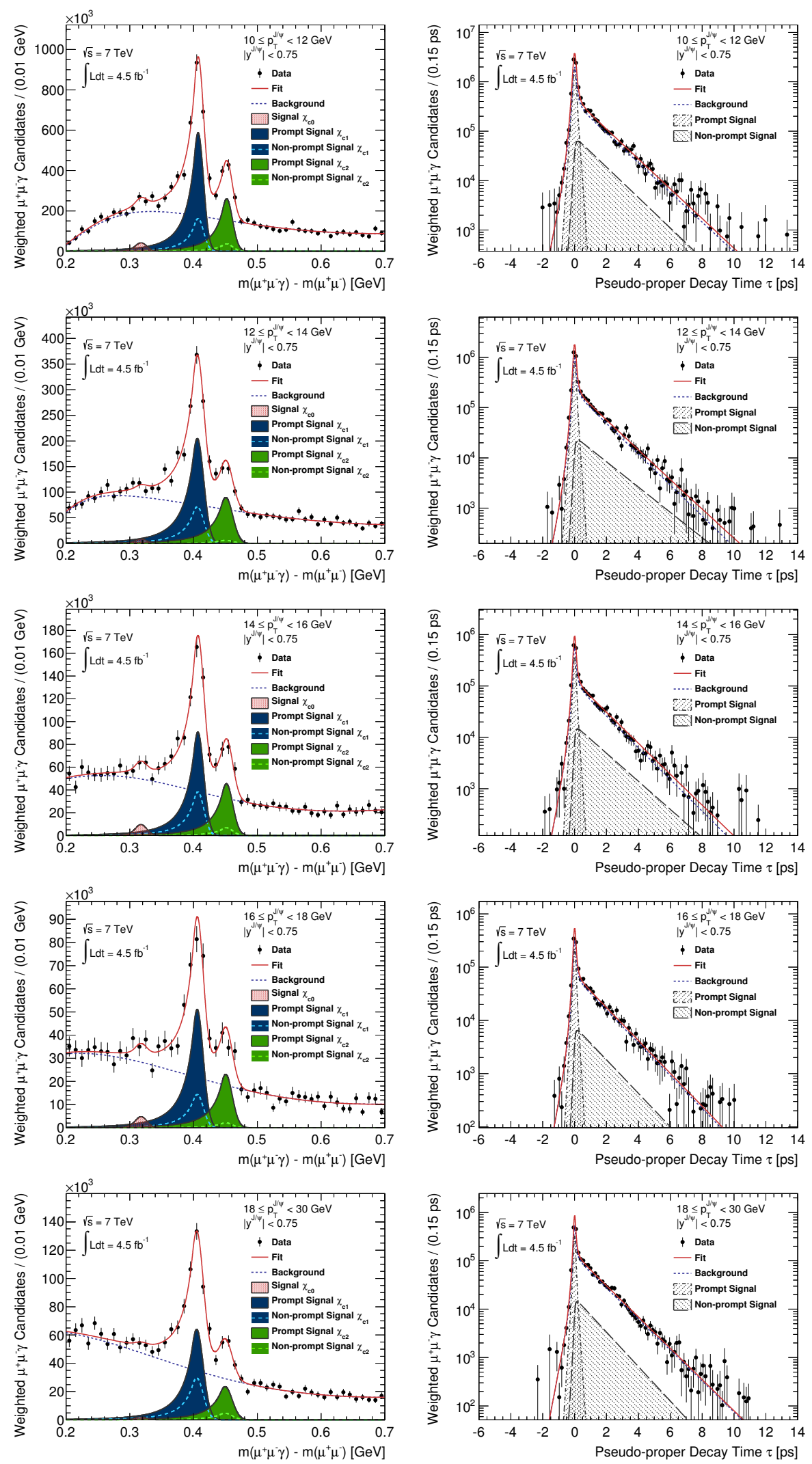

Figure 7.19: The $\Delta m$ and $\tau$ projections of a simultaneous unbinned maximum likelihood fit performed to reconstructed $\chi_{c J}$ decays in bins of $p_{T}^{J / \psi}$. The data are weighted with the prompt $\chi_{c 1}$ acceptance correction. 

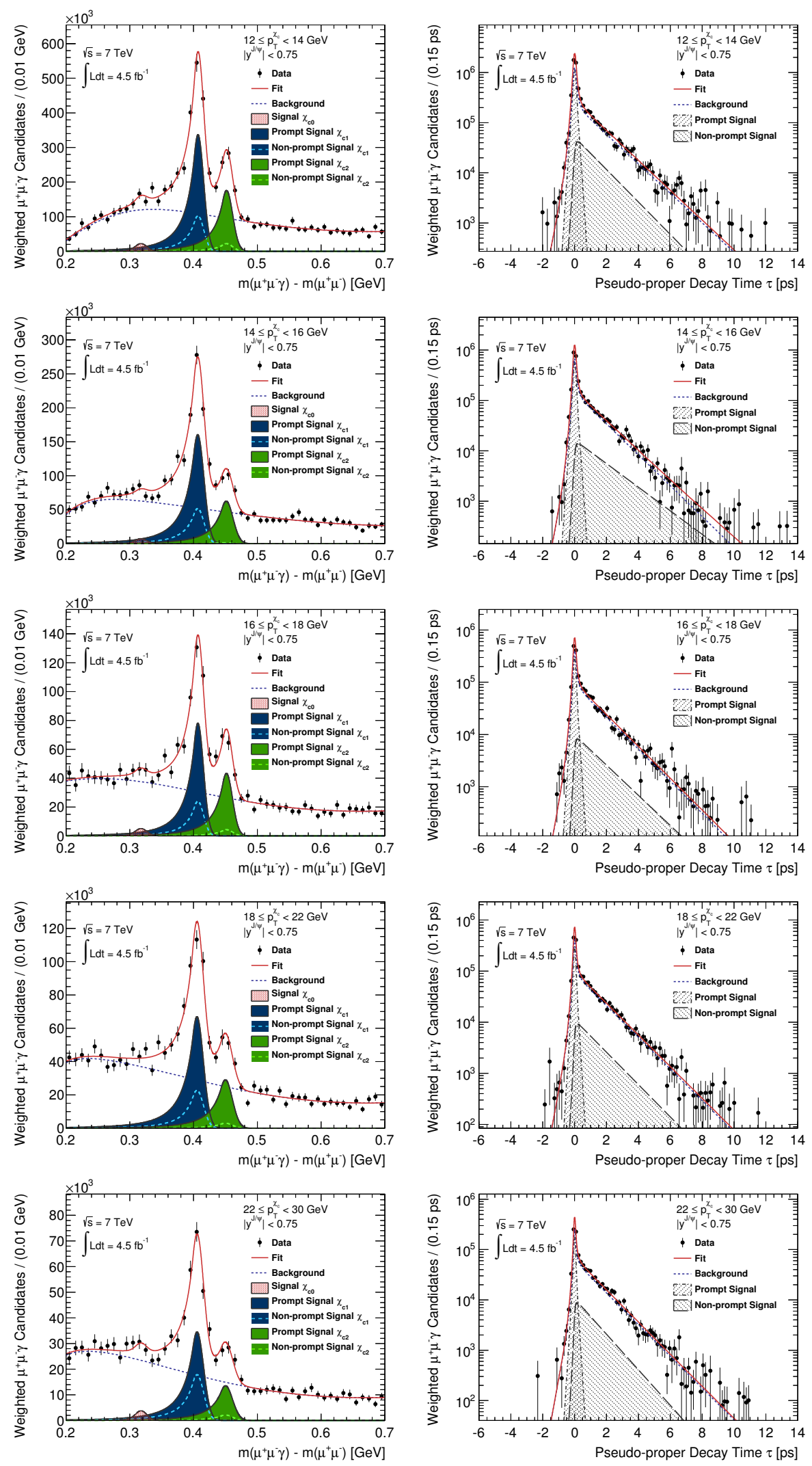

Figure 7.20: The $\Delta m$ and $\tau$ projections of a simultaneous unbinned maximum likelihood fit performed to reconstructed $\chi_{c J}$ decays in bins of $p_{T}^{\chi_{c}}$. The data are weighted with the prompt $\chi_{c 1}$ acceptance correction. 


\subsection{Bin Migration Corrections}

The final stage in obtaining $\chi_{c}$ cross sections is a correction for the effects of experimental resolution in $p_{T}^{\chi_{c}}$ and $p_{T}^{J / \psi}$ on the measured differential cross sections. The finite resolution in both $p_{T}^{\chi_{c}}$ and $p_{T}^{J / \psi}$ causes a net migration of events from their "true" $p_{T}$ bin to adjacent "measured" bins. This effect can arise for even a symmetric resolution function due to the rapidly varying shape of the $p_{T}$ distributions. Bin migrations in $p_{T}^{J / \psi}$ are a small effect since the resolution in $p_{T}^{J / \psi}$ is symmetric and very narrow relative to the $p_{T}^{J / \psi}$ bin sizes (between $2 \mathrm{GeV}$ and $12 \mathrm{GeV}$ ). However, bin migration effects are significantly larger in the case of the cross sections measured as a function of $p_{T}^{\chi_{c}}$, since the $p_{T}^{\chi_{c}}$ resolution function is asymmetric due to the effects of electron energy loss.

The experimental resolutions in $p_{T}^{\chi_{c}}$ and $p_{T}^{J / \psi}$ determined from MC simulation for the fiducial region used in this analysis are shown in Figure 7.21 and are both fitted with $\mathrm{CB}$ functions. The distribution of the ratio of reconstructed $p_{T}$ to true $p_{T}, p_{T}^{\text {reco }} / p_{T}^{\text {truth }}$, should peak at unity with an infinitesimally small width for a perfect detector. The mean of the resolution function for $p_{T}^{J / \psi}$ is consistent with unity while the width is around $1 \%$. The resolution function for $p_{T}^{\chi_{c}}$ also has a width of around $1 \%$ but the mean value of the CB function (0.994) is significantly less than unity and the resolution exhibits a clear tail for $p_{T}^{\text {reco }} / p_{T}^{\text {truth }}<1$, due to electron energy losses in reconstructed photons conversions.

The bin migration correction is determined with a semi-analytical technique designed to unfold smoothly varying distributions [77]. The method involves fitting the acceptance and efficiency corrected $p_{T}^{\chi_{c}}$ and $p_{T}^{J / \psi}$ distributions with an analytic function that is convolved with the resolution functions (parametrised as CB functions) shown in Figure 7.21. The underlying $p_{T}$ distributions, free from resolution effects, are described by the empirically motivated analytical function (as used in Ref. [87]) 


$$
f(x)=\frac{A \cdot x \cdot\left(1+B \cdot x^{E}\right)}{\left(x^{2}+C^{2}\right)^{D}},
$$

where all five parameters $(A-E)$ are freely determined in the fit. Once the measured $p_{T}^{\chi_{c}}$ and $p_{T}^{J / \psi}$ distributions are fitted with the "smeared" function (Eqn. 7.14 numerically convolved with the corresponding CB function shown in Figure 7.21), the underlying "un-smeared" distribution (Eqn. 7.14 as shown, without any convolution) can be accessed directly. The ratio of the fitted "un-smeared" function to the "smeared" function represents a scale factor that can be applied to each measured $p_{T}^{\chi_{c}}$ and $p_{T}^{J / \psi}$ bin to correct for bin migrations caused by experimental resolution. Figures 7.22 and 7.23 show the fitted $p_{T}^{\chi_{c}}$ and $p_{T}^{J / \psi}$ distributions, respectively, in addition to the derived bin migration corrections. The correction factors for the cross sections binned in $p_{T}^{J / \psi}$ are small, at around 0.995 , while the correction factors for the cross sections binned in $p_{T}^{\chi_{c}}$ are larger, at around 1.04, due to the asymmetric resolution in $p_{T}^{\chi_{c}}$. 

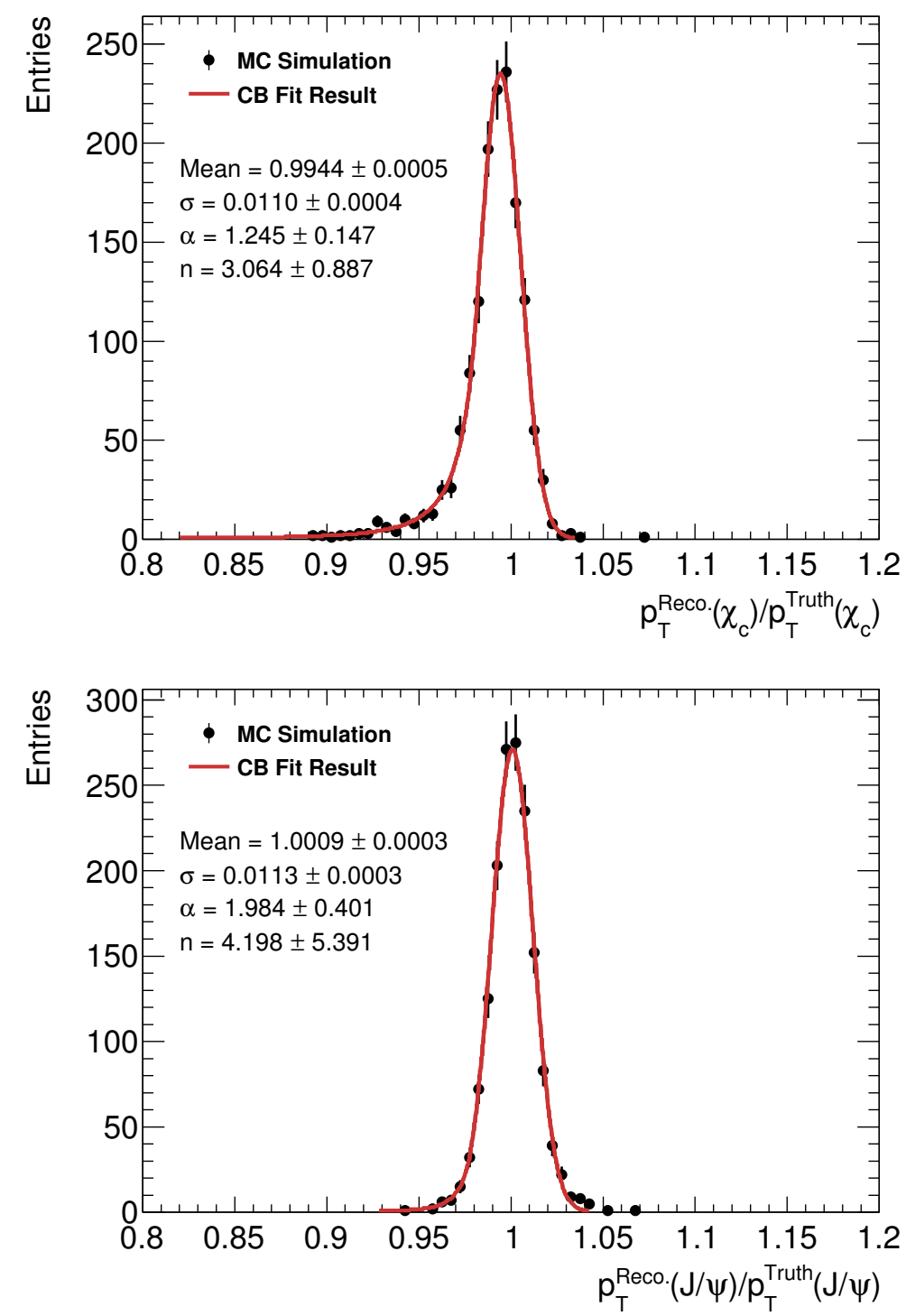

Figure 7.21: The experimental resolution in $p_{T}^{\chi_{c}}$ (top) and $p_{T}^{J / \psi}$ (bottom) for the fiducial region $10<p_{T}^{J / \psi}<30 \mathrm{GeV}$ and $\left|y^{J / \psi}\right|<0.75$, derived from MC simulation. Both distributions are fitted with a CB function. 

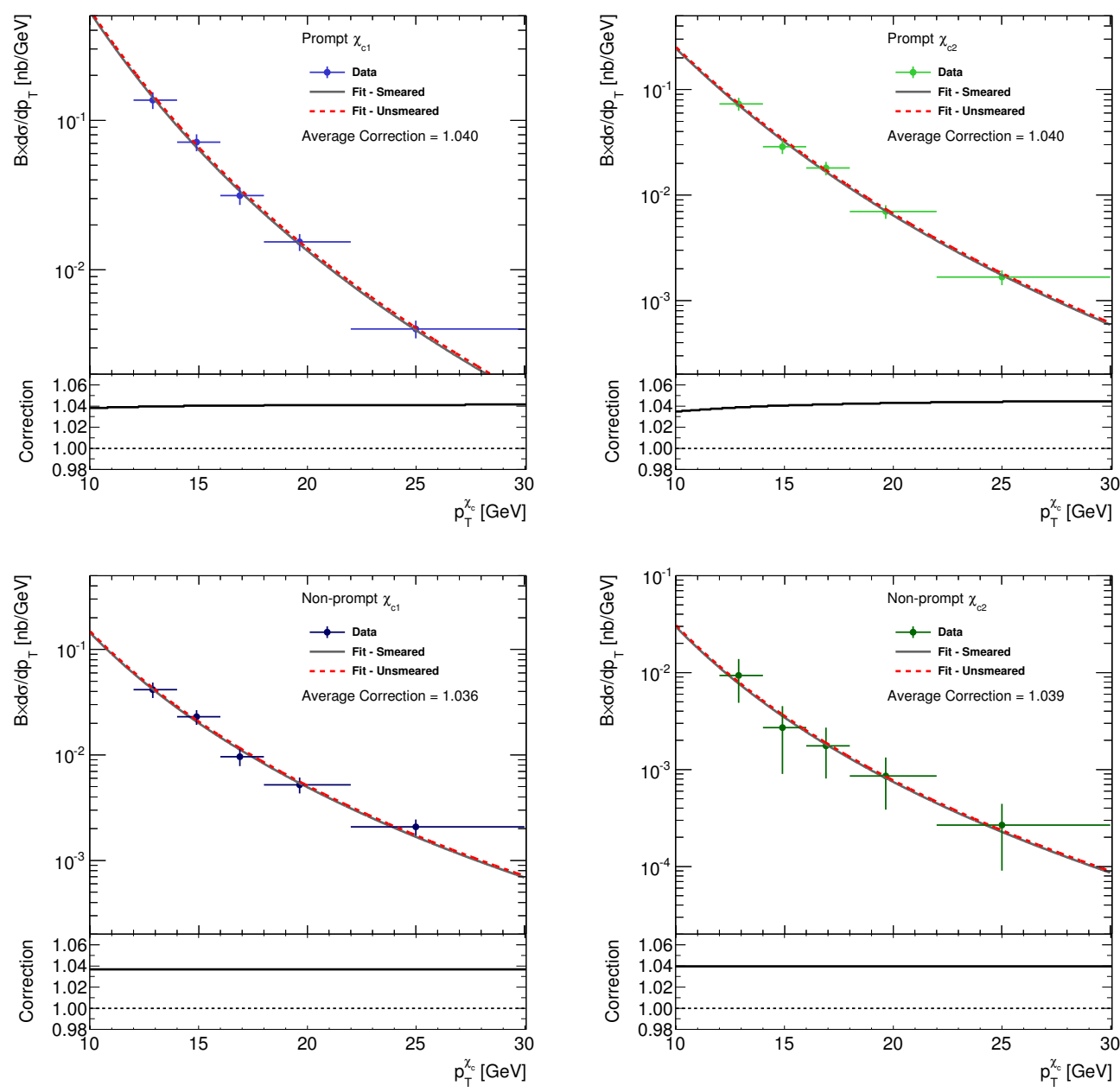

Figure 7.22: Measured $p_{T}^{\chi_{c}}$ distributions for prompt and non-prompt $\chi_{c 1}$ and $\chi_{c 2}$, fitted with an analytical function convolved with a resolution function (solid grey line). The de-convolved function is shown as the red dashed line. The bin migration corrections are shown in the panels below the fitted $p_{T}^{\chi_{c}}$ distributions. 

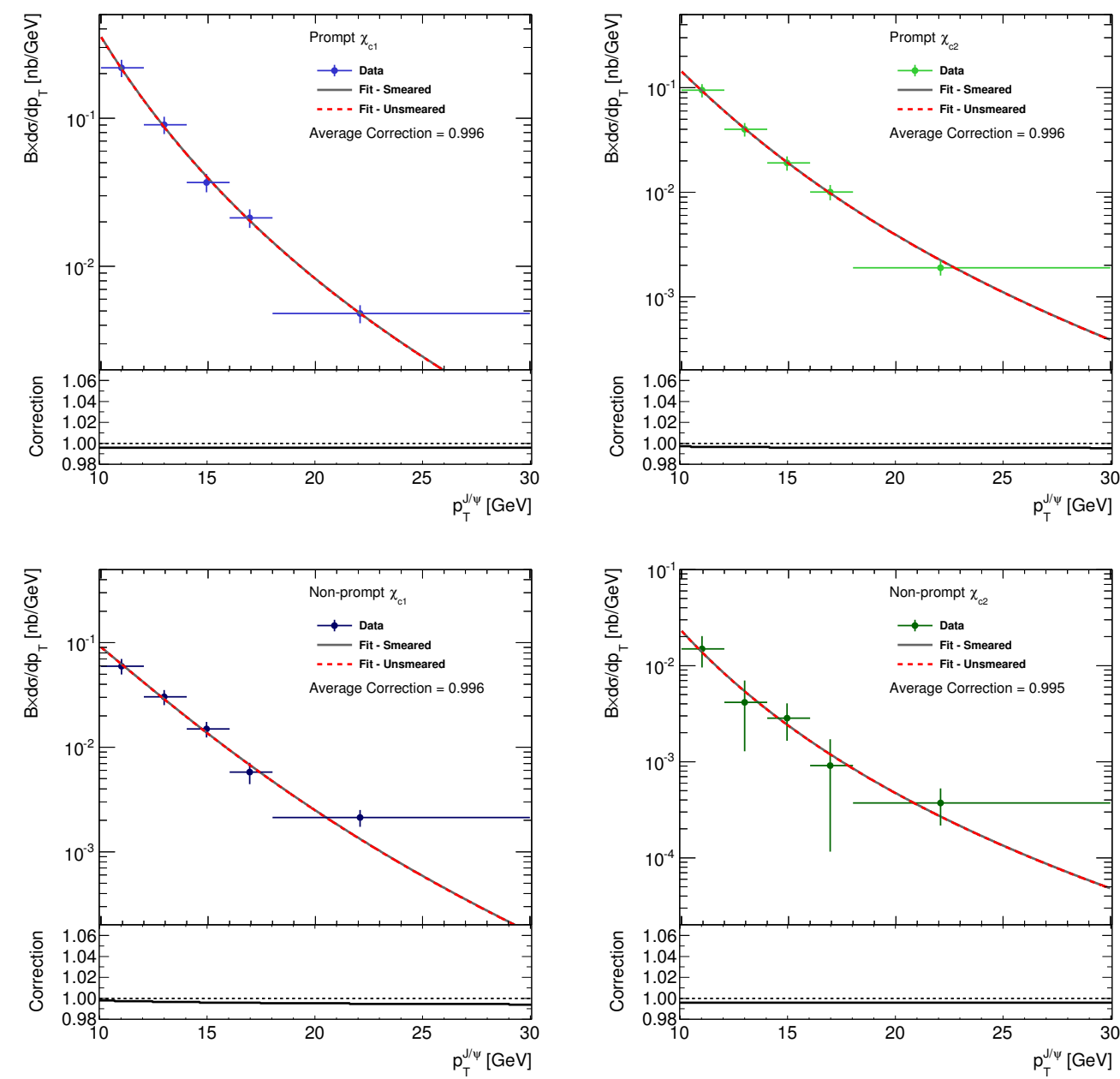

Figure 7.23: Measured $p_{T}^{J / \psi}$ distributions for prompt and non-prompt $\chi_{c 1}$ and $\chi_{c 2}$, fitted with an analytical function convolved with a resolution function (solid grey line). The de-convolved function is shown as the red dashed line. The bin migration corrections are shown in the panels below the fitted $p_{T}^{J / \psi}$ distributions. 


\subsection{Systematic Uncertainties}

Several sources of systematic uncertainty on the measurements of the prompt and non-prompt $\chi_{c 1}$ and $\chi_{c 2}$ cross sections are considered. These uncertainties and the methods used to quantify them are discussed below.

The finite size of each of the data or simulation samples used to derive the efficiency corrections discussed in Section 7.5 gives rise to a statistical uncertainty in each efficiency. A common approach is adopted to quantify the effect of these statistical uncertainties on the measured cross sections. An ensemble of 500 independent alternative efficiency maps are generated by taking the nominal efficiency map and perturbing each bin by a random amount sampled from a Gaussian distribution, with a mean value of zero and a width equal to the statistical uncertainty in that bin. The RMS of the distribution of the average efficiency weight in each $p_{T}^{\chi_{c}}$ and $p_{T}^{J / \psi}$ bin, calculated from the ensemble of alternative efficiency maps, is used as an estimate of the systematic uncertainty.

Acceptance: The method used to correct for the effects of experimental resolution in the calculation of the per-candidate acceptance binned in $p_{T}^{\chi_{c}}$ has an associated uncertainty of $\pm 2 \%$, derived from the envelope of the residual bias from simulation studies. The acceptance maps binned in $p_{T}^{J / \psi}$ are dependent on the $p_{T}^{\chi_{c}}$ distribution used to seed the simulation. To estimate the sensitivity of these acceptance corrections to the $p_{T}^{\chi_{c}}$ distribution used as an input, the measured $p_{T}^{\chi_{c}}$ distributions are refitted with an alternative analytical function. These alternative fitted parametrisations lead to changes in the acceptance binned in $p_{T}^{J / \psi}$ of between $4-8 \%$ of the nominal values. This range is adopted as an estimate of the systematic uncertainty on the acceptance corrections binned in $p_{T}^{J / \psi}$ due to the $p_{T}^{\chi_{c}}$ distribution used as input. The statistical uncertainty in the acceptance corrections, in comparison to the effects mentioned above, is negligible. 
Muon Identification Efficiency: The systematic uncertainty on the muon identification efficiency is composed of two components. The first component is a statistical uncertainty and is quantified as described earlier. The second component is an uncertainty associated with the fitting procedure used to measure $J / \psi \rightarrow \mu^{+} \mu^{-}$ yields in the tag-and-probe analysis used to derive the efficiencies. The sum in quadrature of these two uncertainties is around $\pm 1 \%$.

Muon Track Reconstruction Efficiency: The systematic uncertainty on the track reconstruction efficiency for muons is estimated to be $\pm 1 \%$ from studies performed in Ref. [87].

Trigger Efficiency: The systematic uncertainty on the trigger efficiency is composed of three components. The first two components are identical in nature to those discussed for the muon identification efficiency; a statistical uncertainty and a systematic uncertainty on the fitting procedure used to measure the efficiency with a $J / \psi \rightarrow \mu^{+} \mu^{-}$tag-and-probe analysis in data. The third component of the uncertainty is related to the fitted parametrisation of the $\Delta R$ dependence of the $c_{\mu^{+} \mu^{-}}$correction. This uncertainty is shown as the $\pm 1 \sigma$ variations of $c_{\mu^{+} \mu^{-}}(\Delta R)$ in Figure 7.8. The changes in the average weight calculated with the $\pm 1 \sigma$ variations of $c_{\mu^{+} \mu^{-}}(\Delta R)$ are used to estimate this component of the systematic uncertainty. The sum of these three components in quadrature represents a total systematic uncertainty on the trigger efficiency correction of around $\pm 4 \%$.

Conversion Probability: The photon conversion probability estimated from MC simulation is sensitive to the total material distribution in the ID in the ATLAS detector simulation. The systematic uncertainty on the conversion probability due to the simulated material distribution is estimated with an alternative set of $\chi_{c} \mathrm{MC}$ simulation samples, which are processed with an alternative detector material distribution that contains around $20 \%$ more inactive material (typically services such as cooling) between the pixel layers of the detector (an increase based upon a conservative assessment of the known material distribution). The systematic uncertainty 
in the photon conversion probability estimated from these alternative simulation samples is $\pm 4 \%$.

Conversion Reconstruction Efficiency: Several effects contribute to the systematic uncertainty on the estimate of the conversion reconstruction efficiency from MC simulation. The dominant source is the uncertainty in the behaviour of the ATLAS conversion finding algorithm in the MC simulation, compared to data. Several sensitive distributions of conversion observables (vertex position, fit $\chi^{2} /[$ d.o.f.], number of track hits etc.) are compared for an inclusive (i.e not necessarily from $\chi_{c J}$ decays) sample of photon conversions in data and MC simulation. The features of these distributions in data are well reproduced by the $\mathrm{MC}$ simulation and any residual discrepancies are bounded by a conservative $\pm 10 \%$ envelope. Since any discrepancies in these distributions could also be due to the material model, the estimate of the systematic uncertainty due to these residual discrepancies is reduced to $\pm 9 \%$, based on the uncertainty in the detector material distribution discussed above. The other large contribution to the systematic uncertainty on the estimate of the conversion reconstruction efficiency comes from the uncertainty in the method described in Section 7.5.4 to avoid biases due to the experimental resolution in $p_{T}^{\gamma}$. This uncertainty is estimated to be $\pm 5 \%$ from simulation studies, including the self-consistency tests shown in Section 7.5.4. The dependence of the efficiency on the kinematic distributions of the $\chi_{c J}$ decays in the simulated $\chi_{c J}$ event samples is assessed through re-weighting the $p_{T}^{\gamma}$ distributions and results in changes of around $\pm 1 \%$. The statistical uncertainty in the efficiency corrections, assessed as described above, also contributes around $\pm 2 \%$ to the total uncertainty. The sum in quadrature of these individual components, around $\pm 11 \%$, is taken as an estimate of the total systematic uncertainty on the estimate of the conversion reconstruction efficiency. Fitting Procedure: The systematic uncertainty associated with the fitting procedure is estimated with a toy $\mathrm{MC}$ simulation technique. The nominal fit results in each $p_{T}^{\chi_{c}}$ and $p_{T}^{J / \psi}$ bin are used to generate 200 unique simulated data samples 
for each bin. These simulated data samples are then fitted with several modified fitting procedures to estimate the sensitivity of the fitted yields to variations in the fit model. The following modifications to the fitting procedure are studied:

- The nominal background PDF is replaced by a modified Novosibirsk function [122] with four free parameters.

- Motivated by the fact that the $\chi_{c 0}$ yield is insignificant in most individual $p_{T}^{\chi_{c}}$ and $p_{T}^{J / \psi}$ bins, the fit is repeated with the $\chi_{c 0}$ signal component removed.

- The constraints on the CB parameters $\alpha$ and $n$ of the $\chi_{c 1}$ and $\chi_{c 2}$ signal PDFs are individually released to be determined in the fit.

- The constraint on the scaling between the CB parameter $\sigma$ of the $\chi_{c 1}$ and $\chi_{c 2}$ signal PDFs is released and both $\sigma_{1}$ and $\sigma_{2}$ are determined in the fit.

The systematic uncertainty is then estimated from the mean of the distribution of the relative changes in fitted yield between the nominal and alternative models. The total systematic uncertainty on the fit model is estimated to be in the range $3 \%$ to $9 \%$. This procedure is also performed for fit results for the cross section ratios and non-prompt fractions, to ensure statistical correlations are accounted for in the estimation of the systematic uncertainty.

Integrated Luminosity: The systematic uncertainty in the measurement of the integrated luminosity associated with the data sample is estimated to be $\pm 1.8 \%$. The measurements used to estimate this uncertainty are described in detail in Ref. [110]. This uncertainty cancels directly in all of the cross section ratios measured within the same data sample (i.e. excluding $R_{\chi_{c}}$ alone).

$\chi_{c J}$ Polarisation: The systematic uncertainty on the measurements due to the unknown $\chi_{c J}$ polarisation is estimated from the envelope of the acceptance variations calculated with the polarisation scenarios discussed in Section 7.5.1. The envelope is around ${ }_{-10}^{+30} \%$ for $\chi_{c 1}$ and around ${ }_{-20}^{+30} \%$ for $\chi_{c 2}$. In the case of the cross section 
ratios, the combination of the $\chi_{c 1}$ and $\chi_{c 2}$ polarisation scenarios that gives rise to the maximum variation in the cross section ratios is chosen; $J=1$ helicity \pm 1 combined with $J=2$ helicity \pm 2 gives rise to the upper limit, while $J=1$ helicity 0 combined with $J=2$ helicity 0 gives rise to the lower limit. The envelope for the measurement of $R_{\chi_{c}}$ is calculated assuming overall unpolarised production for prompt $J / \psi$, motivated by the measurements of ALICE, CMS and LHCb [83-85]. These uncertainties are separated from the other systematic uncertainties as they are potentially reducible with a future measurement of the $\chi_{c J}$ polarisation. Scale factors are included in Table A.9 in Appendix A that can be used to modify the measurements to any of the polarisation scenarios discussed in Section 7.5.1.

The individual sources of systematic uncertainty on the measurements of the prompt and non-prompt $\chi_{c 1}$ and $\chi_{c 2}$ cross sections, averaged over $p_{T}$ bins, are shown in Tables 7.3 and 7.4 for the $p_{T}^{\chi_{c}}$ and $p_{T}^{J / \psi}$ binned measurements, respectively. The same information is also shown graphically in Figure 7.24 for both the $p_{T}^{\chi_{c}}$ and $p_{T}^{J / \psi}$ binned measurements.

\begin{tabular}{|l|c|c|c|c|}
\hline \multirow{2}{*}{ Binning: $p_{T}^{\chi_{c}}$} & \multicolumn{4}{|c|}{ Fractional Uncertainty [\%] } \\
\cline { 2 - 5 } & \multicolumn{2}{|c|}{ Prompt } & \multicolumn{2}{c|}{ Non-prompt } \\
\cline { 2 - 5 } & $\chi_{c 1}$ & $\chi_{c 2}$ & $\chi_{c 1}$ & $\chi_{c 2}$ \\
\hline Muon reco. efficiency & 1 & 1 & 1 & 1 \\
Trigger efficiency & 3 & 4 & 4 & 4 \\
Converted-photon reco. efficiency & 11 & 11 & 11 & 11 \\
Conversion probability & 4 & 4 & 4 & 4 \\
Acceptance & 2 & 2 & 2 & 2 \\
Fit model & 2 & 3 & 3 & 8 \\
\hline Total systematic & 12 & 12 & 12 & 14 \\
\hline Polarisation envelope (upper) & 29 & 31 & 29 & 31 \\
Polarisation envelope (lower) & 11 & 20 & 11 & 20 \\
\hline
\end{tabular}

Table 7.3: The individual contributions to the systematic uncertainty on the crosssection measurements binned in $p_{T}^{\chi_{c}}$, averaged across all $p_{T}^{\chi_{c}}$ bins. The common contributions of integrated luminosity (1.8\%) and track reconstruction (1\%) are not shown. The average variation in the cross sections due to the envelope of all possible polarisation scenarios is also shown. 


\begin{tabular}{|l|c|c|c|c|}
\hline \multirow{2}{*}{ Binning: $p_{T}^{J / \psi}$} & \multicolumn{3}{|c|}{ Fractional Uncertainty [\%] } \\
\cline { 2 - 5 } & \multicolumn{2}{|c|}{ Prompt } & \multicolumn{2}{c|}{ Non-prompt } \\
\cline { 2 - 5 } & $\chi_{c 1}$ & $\chi_{c 2}$ & $\chi_{c 1}$ & $\chi_{c 2}$ \\
\hline Muon reco. efficiency & 1 & 1 & 1 & 1 \\
Trigger efficiency & 4 & 4 & 4 & 4 \\
Converted-photon reco. efficiency & 11 & 11 & 11 & 11 \\
Conversion probability & 4 & 4 & 4 & 4 \\
Acceptance & 4 & 4 & 5 & 8 \\
Fit model & 2 & 3 & 3 & 9 \\
\hline Total systematic & 13 & 13 & 13 & 17 \\
\hline Polarisation envelope (upper) & 34 & 36 & 32 & 36 \\
Polarisation envelope (lower) & 13 & 23 & 13 & 23 \\
\hline
\end{tabular}

Table 7.4: The individual contributions to the systematic uncertainty on the crosssection measurements binned in $p_{T}^{J / \psi}$, averaged across all $p_{T}^{J / \psi}$ bins. The common contributions of integrated luminosity (1.8\%) and track reconstruction (1\%) are not shown. The average variation in the cross sections due to the envelope of all possible polarisation scenarios is also shown. 

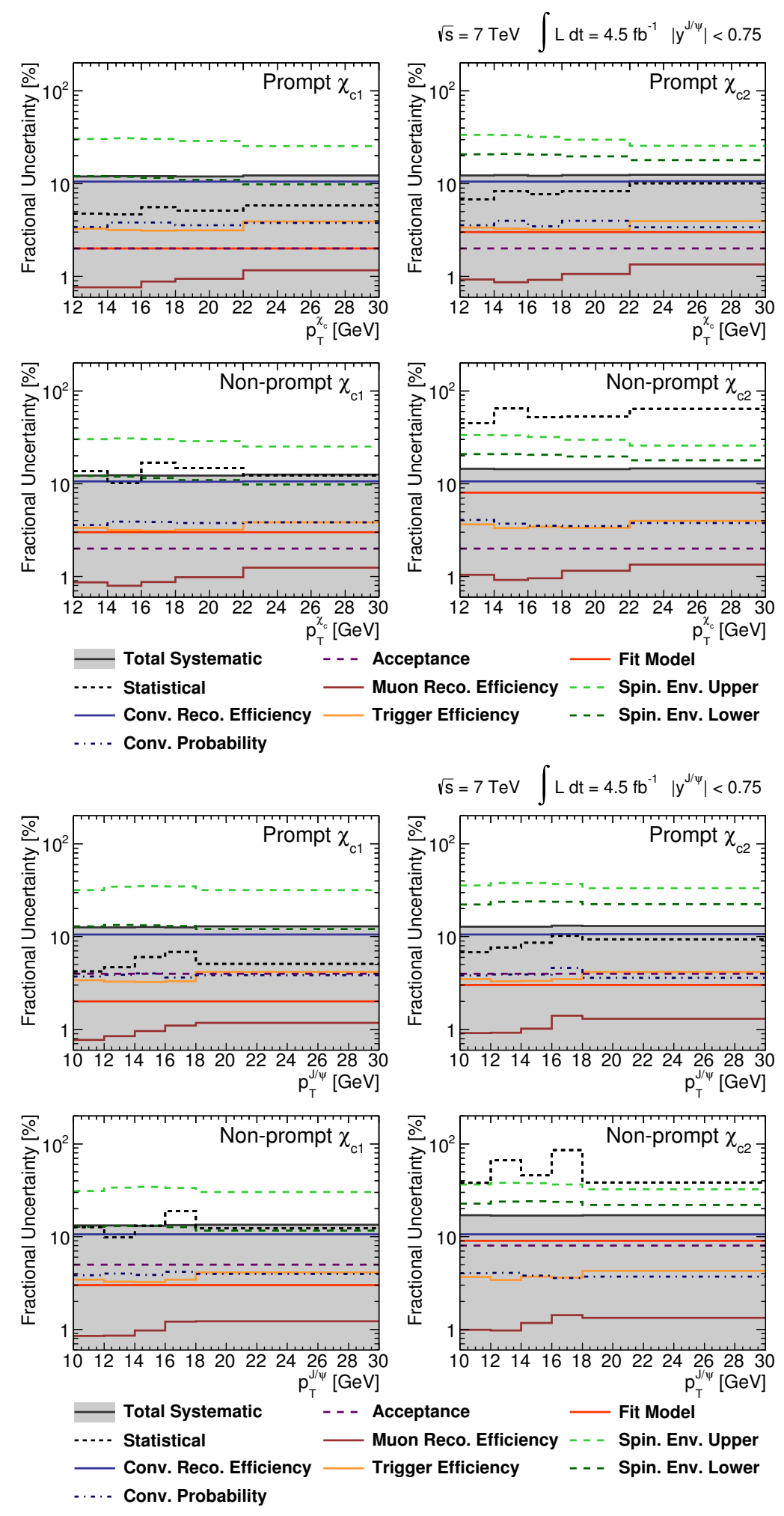

Figure 7.24: The individual contributions to the systematic uncertainty on the crosssection measurements binned in $p_{T}^{\chi_{c}}$ (top) and $p_{T}^{J / \psi}$ (bottom). The common contributions of integrated luminosity $(1.8 \%)$ and track reconstruction (1\%) are not shown. The lines denoted Spin. Env. represent the upper and lower bounds of the polarisation envelope (not included in the total systematic uncertainty). 


\subsection{Results and Discussion}

The differential cross sections for prompt and non-prompt $\chi_{c 1}$ and $\chi_{c 2}$ measured as a function of both $p_{T}^{\chi_{c}}$ and $p_{T}^{J / \psi}$, in addition to several cross section ratios and associated quantities are shown in Figures 7.25 to 7.32 . These measurements are compared to existing measurements and to theoretical predictions where appropriate. Tabulated results are also included in Appendix A.

\subsubsection{Theoretical Predictions}

The measurements of $\chi_{c 1}$ and $\chi_{c 2}$ production are compared to several theoretical predictions based on QCD. Each of these theoretical predictions is described below. All of the theoretical predictions are scaled by the world average values of the branching fractions $\mathcal{B}\left(\chi_{c J} \rightarrow J / \psi \gamma\right)$ and $\mathcal{B}\left(J / \psi \rightarrow \mu^{+} \mu^{-}\right)$(see Table 7.1) [3].

\section{NRQCD}

The direct $\chi_{c J}$ production cross sections have been calculated at next-to-leading order (NLO) within the NRQCD factorisation approach [153,154], which is described in detail in Section 3.2.5. The calculation in $[153,154]$ uses a set of LDMEs extracted from fits to charmonium production data from the Tevatron; the details of this procedure are described in [155]. The shaded uncertainty bands of the NRQCD predictions, shown in Figures 7.25 and 7.26, are derived from factorisation and renormalisation scale uncertainties, in addition to a contribution from the extraction of NRQCD long distance matrix elements from data.

\section{The Colour Singlet Model}

The CHIGEN MC event generator is used to produce a prediction for the direct $\chi_{c J}$ production cross sections calculated within the CSM [156], which is described in detail in Section 3.2.3. The partonic sub-processes $g g \rightarrow \chi_{c J} g$ represent the 
dominant contributions to the production cross sections, within the fiducial region studied. The process $g g \rightarrow \chi_{c 2}$ also provides a small contribution to $\chi_{c 2}$ production at lower $p_{T}$ (this process is forbidden for $\chi_{c 1}$ ). The shaded uncertainty bands of the CSM predictions, shown in Figures 7.25 and 7.26, are derived from factorisation and renormalisation scale uncertainties.

\section{The $k_{\mathrm{T}}$ factorisation approach}

The $k_{\mathrm{T}}$ factorisation approach combines the CSM prediction for the direct production of $\chi_{c J}$ with PDFs that contain an explicit $p_{T}$ dependence to deduce an independent prediction $[157,158]$, as described in Section 3.2.4.

\section{Fixed order next-to-leading logarithm $b$-hadron production}

A theoretical prediction for $\chi_{c J}$ production in $b$-hadron decays is formed from a prediction for for $B^{ \pm, 0}$ meson production within the fixed-order next-to-leading logarithm (FONLL) approach [56]. Due to the limited data available for inclusive $b$-hadron decays to $\chi_{c J}$ states, this prediction implicitly assumes that the decay behaviour (inclusive branching fractions and decay product momentum distributions) of the mixture of $b$-hadrons produced at the LHC can be approximated to that of $B^{ \pm, 0}$ mesons (i.e. that all $b$-quarks fragment into $B^{ \pm, 0}$ mesons alone). The inclusive decay of the $B^{ \pm, 0}$ mesons to final states including $\chi_{c 1}$ and $\chi_{c 2}$ states is modelled with the $p^{*}$ (the momentum of the $\chi_{c J}$ in the $B$ rest frame) distributions measured by BaBar [60]. The predictions are scaled with the current world-average values for the branching fractions $\mathcal{B}\left(B^{ \pm / 0} \rightarrow \chi_{c 1} X\right)=(3.86 \pm 0.27) \times 10^{-3}$ and $\mathcal{B}\left(B^{ \pm / 0} \rightarrow \chi_{c 2} X\right)=(1.3 \pm 0.4) \times 10^{-3}[3]$. The shaded uncertainty band on the FONLL predictions, shown in Figures 7.27 and 7.28, represents the theoretical uncertainty due to factorisation and renormalisation scales, quark masses and parton distribution functions, combined with the uncertainty on the branching fractions used to scale the predictions. 

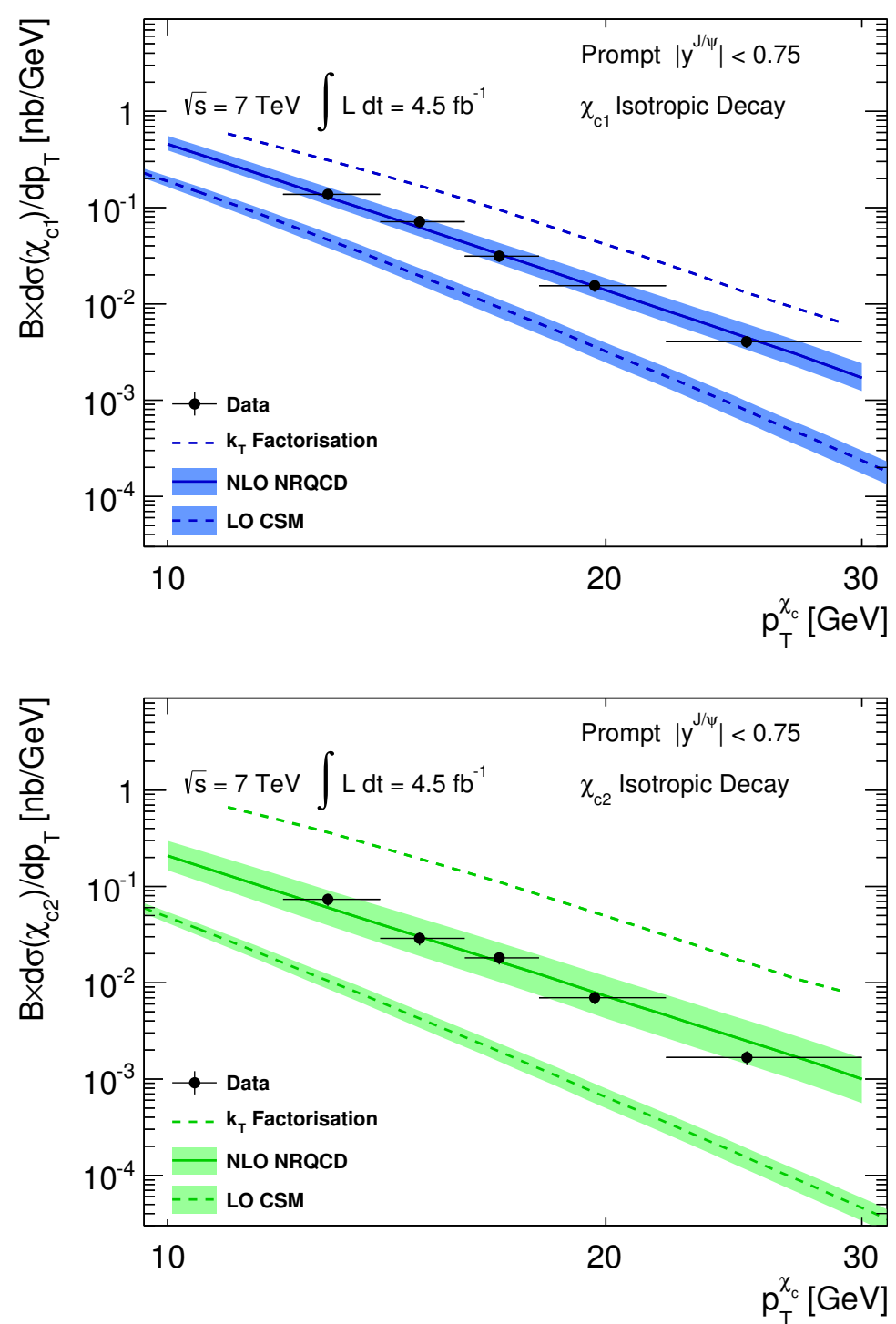

Figure 7.25: Differential cross sections for prompt $\chi_{c 1}$ (top) and $\chi_{c 2}$ (bottom) measured as a function of $p_{T}^{\chi_{c}}$. The measurements are compared to the predictions of NLO NRQCD $[153,154]$, the CSM [156] and the $k_{\mathrm{T}}$ factorisation approach $[157,158]$. The positions of the data points within each bin reflect the average $p_{T}^{\chi_{c}}$ of the $\chi_{c}$ candidates within the bin. The error bars represent the total uncertainty on the measurement (statistical and systematic), assuming isotropic decay angular distributions (in some cases, the error bar is smaller than the data point). 

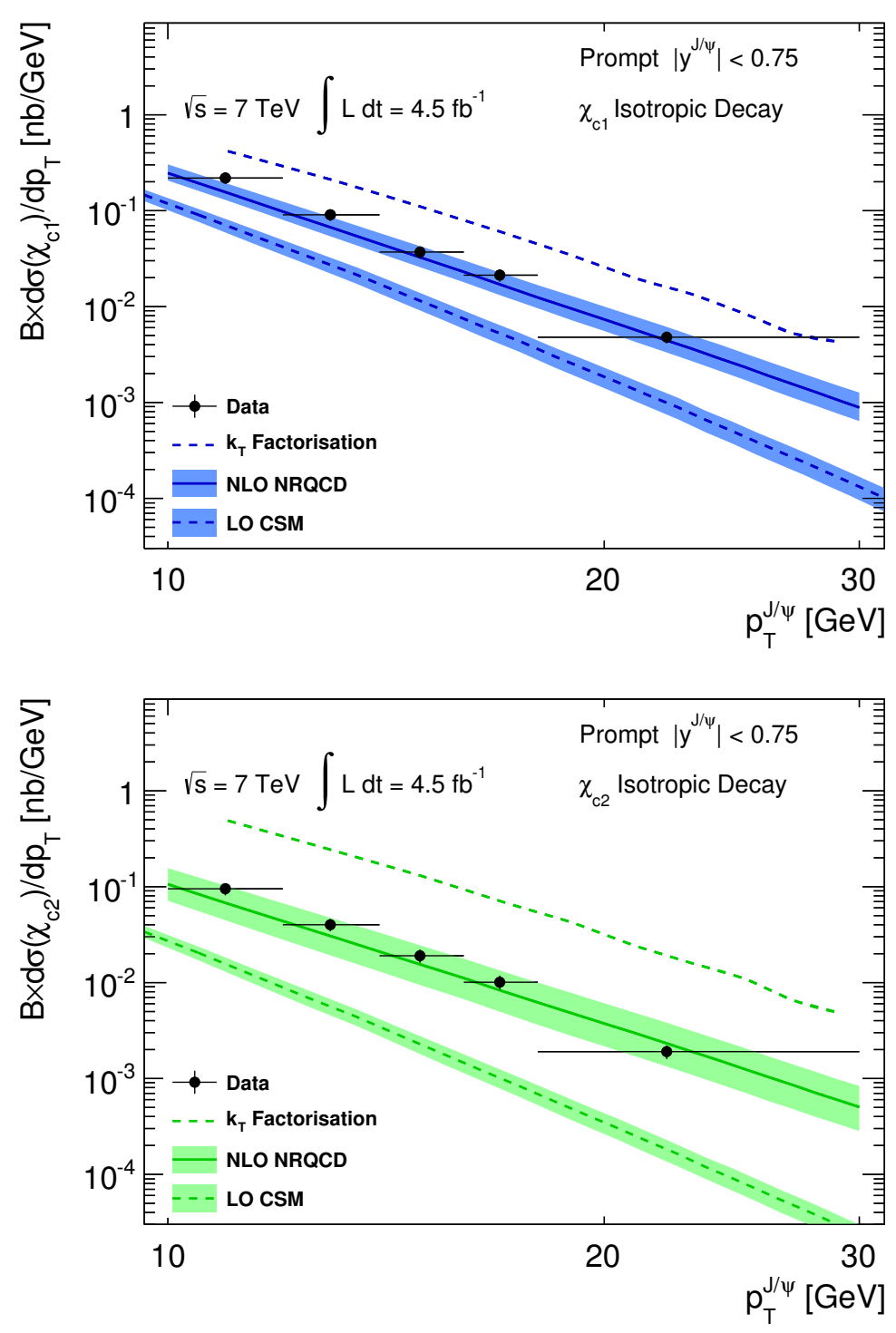

Figure 7.26: Differential cross sections for prompt $\chi_{c 1}$ (top) and $\chi_{c 2}$ (bottom) measured as a function of $p_{T}^{J / \psi}$. The measurements are compared to the predictions of NLO NRQCD [153,154], the CSM [156] and the $k_{\mathrm{T}}$ factorisation approach $[157,158]$. The positions of the data points within each bin reflect the average $p_{T}^{J / \psi}$ of the $\chi_{c}$ candidates within the bin. The error bars represent the total uncertainty on the measurement (statistical and systematic), assuming isotropic decay angular distributions (in some cases, the error bar is smaller than the data point). 


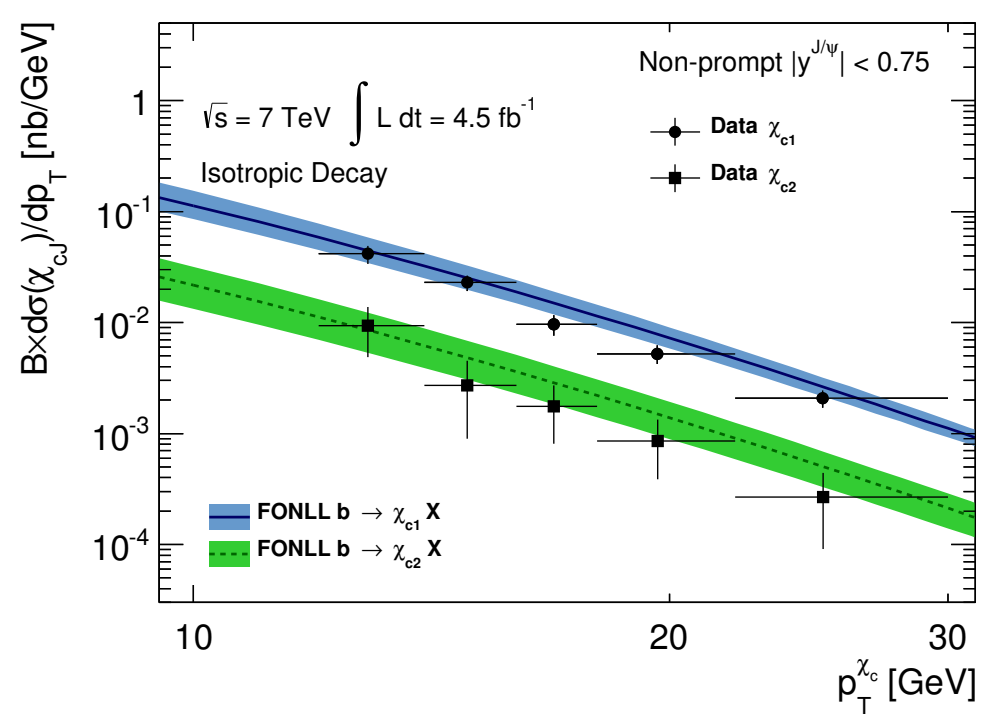

Figure 7.27: Differential cross sections for non-prompt $\chi_{c 1}$ (blue) and $\chi_{c 2}$ (green) measured as a function of $p_{T}^{\chi_{c}}$. The measurements are compared to the predictions of a theoretical prediction based on the FONLL model of $b$ hadron production [56]. The positions of the data points within each bin reflect the average $p_{T}^{\chi_{c}}$ of the $\chi_{c}$ candidates within the bin. The error bars represent the total uncertainty on the measurement (statistical and systematic), assuming isotropic decay angular distributions.

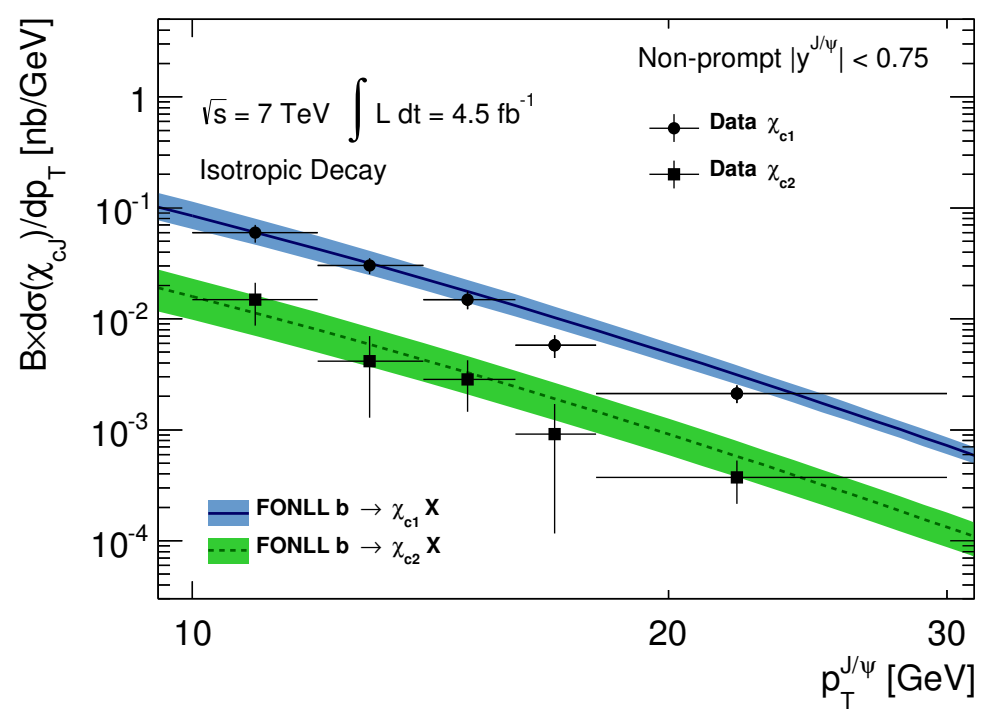

Figure 7.28: Differential cross sections for non-prompt $\chi_{c 1}$ (blue) and $\chi_{c 2}$ (green) measured as a function of $p_{T}^{J / \psi}$. The measurements are compared to the predictions of a theoretical prediction based on the FONLL model of $b$ hadron production [56]. The positions of the data points within each bin reflect the average $p_{T}^{J / \psi}$ of the $\chi_{c}$ candidates within the bin. The error bars represent the total uncertainty on the measurement (statistical and systematic), assuming isotropic decay angular distributions. 


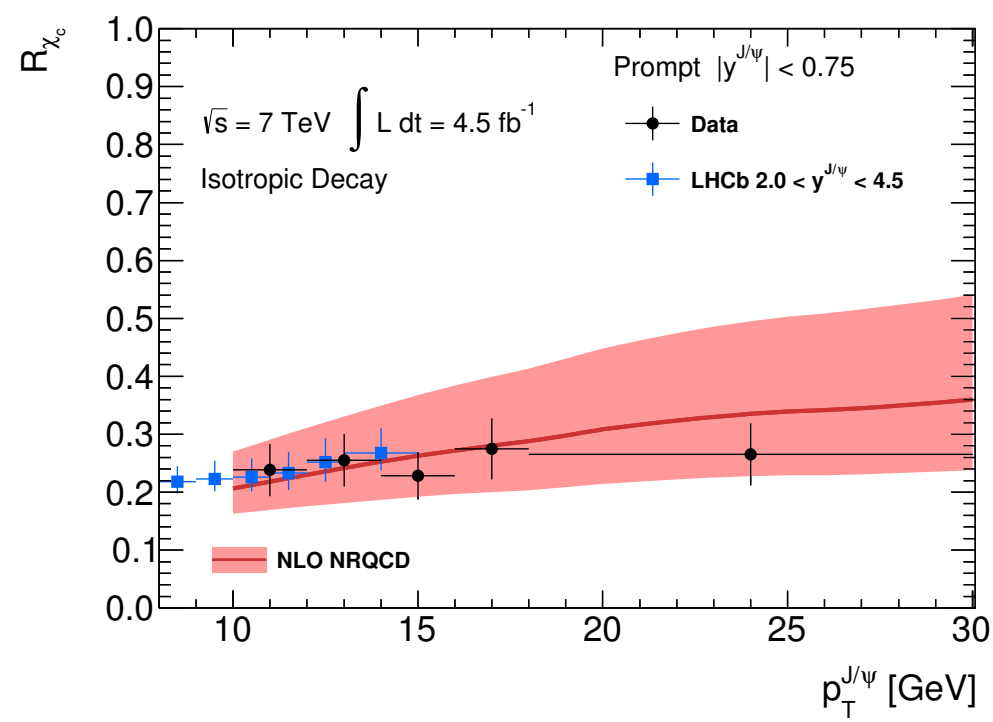

Figure 7.29: The fraction of prompt $J / \psi$ produced in feed-down from radiative $\chi_{c J}$ decays, measured as a function of $p_{T}^{J / \psi}$. The measurements are compared to the predictions of NLO NRQCD [153,154] and the measurement of LHCb [82]. The error bars represent the total uncertainty on the measurement (statistical and systematic), assuming isotropic decay angular distributions.

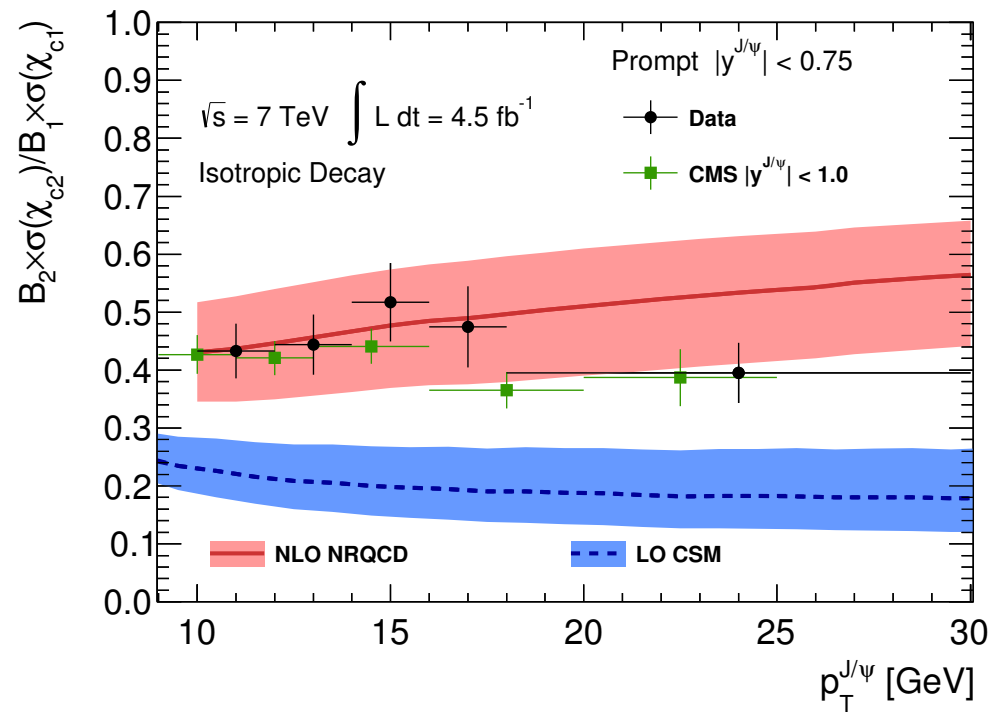

Figure 7.30: The cross section of prompt $\chi_{c 2}$ relative to prompt $\chi_{c 1}$, measured as a function of $p_{T}^{J / \psi}$. The measurements are compared to the predictions of NLO NRQCD [153,154] and the CSM [156] and the measurement of CMS [40]. The error bars represent the total uncertainty on the measurement (statistical and systematic), assuming isotropic decay angular distributions. 


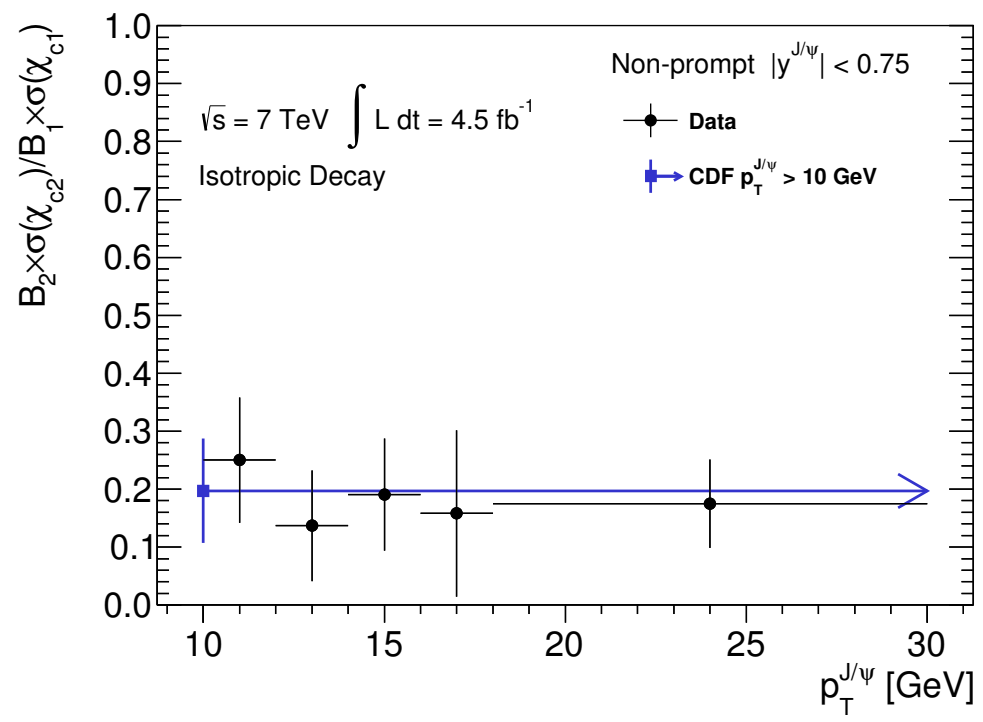

Figure 7.31: The cross section of non-prompt $\chi_{c 2}$ relative to non-prompt $\chi_{c 1}$, measured as a function of $p_{T}^{J / \psi}$. The measurements are compared to that of CDF in $p \bar{p}$ collisions at $\sqrt{s}=1.96 \mathrm{TeV}$ [38]. The error bars represent the total uncertainty on the measurement (statistical and systematic), assuming isotropic decay angular distributions.

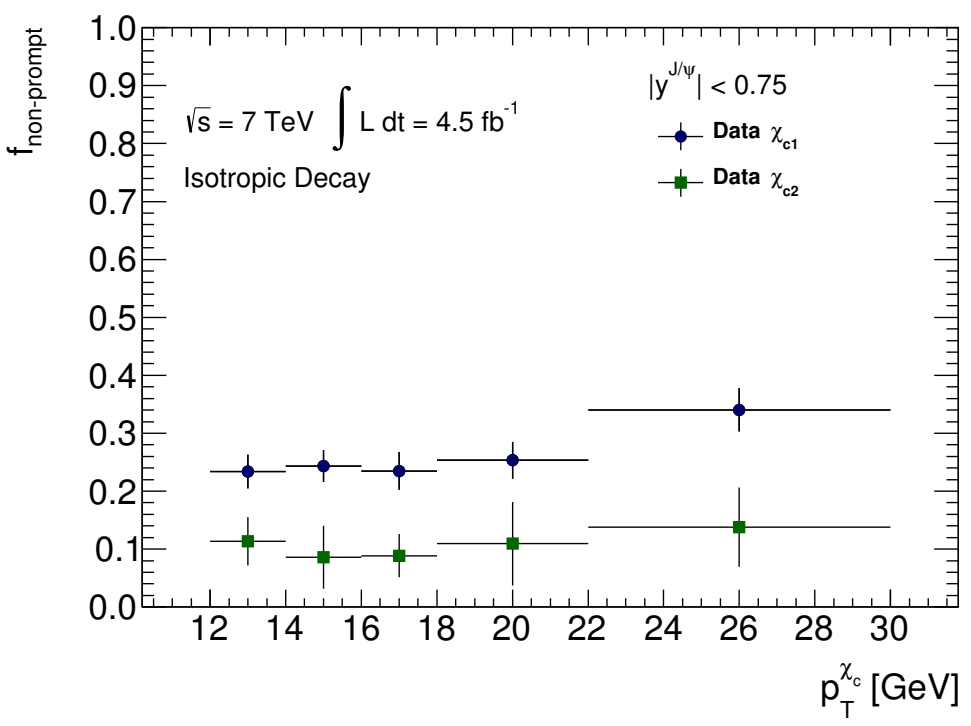

Figure 7.32: The fraction of $\chi_{c 1}$ and $\chi_{c 2}$ produced in the decays of $b$-hadrons measured as a function of $p_{T}^{\chi_{c}}$. The error bars represent the total uncertainty on the measurement (statistical and systematic), assuming isotropic decay angular distributions. 


\subsubsection{Prompt differential cross sections}

The prompt production cross sections of $\chi_{c 1}$ and $\chi_{c 2}$ states, measured as functions of $p_{T}^{\chi_{c}}$ and $p_{T}^{J / \psi}$, are shown in Figures 7.25 and 7.26, respectively. The predictions of NLO NRQCD are in good agreement with the measurements across the $p_{T}^{\chi_{c}}$ and $p_{T}^{J / \psi}$ ranges studied. The NLO NRQCD predictions have also been shown to describe the measured $\chi_{c J}$ production cross sections at the Tevatron with a similar level of agreement [153]. The predictions of the $k_{T}$ factorisation approach for the prompt $\chi_{c 1}$ and $\chi_{c 2}$ cross sections are significantly in excess of the measurements. The $k_{T}$ factorisation approach has been shown to reproduce the overall prompt $J / \psi$ cross section (the model includes $\chi_{c}$ and $\psi(2 S)$ feed-down) [157]. However, recent measurements of $\psi(2 S)$ production show that the same prediction significantly underestimates prompt $\psi(2 S)$ production [159]. While the $k_{T}$ factorisation approach may reproduce the total prompt $J / \psi$ cross section, it does not seem to predict accurately the measured composition (i.e. the relative amounts of direct $J / \psi$ and feed-down from $\chi_{c}$ and $\psi(2 S)$ decays). The prediction of the CSM is significantly below the measured cross sections, which may suggest that colour octet contributions or higher order perturbative corrections, missing in this prediction, are necessary to describe the measured cross sections.

\subsubsection{Fraction of prompt $J / \psi$ produced in $\chi_{c J}$ feed-down de- cays: $R \chi_{c}$}

The differential cross sections for prompt $\chi_{c 1}$ and $\chi_{c 2}$, measured as functions of $p_{T}^{J / \psi}$, are summed and presented as a fraction of the prompt $J / \psi$ differential cross section measured by ATLAS [77]. This quantity, known as $R_{\chi_{c}}$, represents the fraction of prompt $J / \psi$ produced in feed-down from radiative $\chi_{c}$ decays, neglecting the very small contribution from radiative $\chi_{c 0}$ decays. If one assumes an equal production rate for $\chi_{c 0}$ and $\chi_{c 2}$, (motivated by the LHCb measurement $\sigma_{0} / \sigma_{2}=$ 
$1.19 \pm 0.40[149])$, then radiative $\chi_{c 0}$ decays represent around $2 \%$ of the combined $\chi_{c J}$ contribution to prompt $J / \psi$ production. Figure 7.29 shows that $24-28 \%$ of prompt $J / \psi$ are produced in radiative $\chi_{c J}$ decays for $10<p_{T}^{J / \psi}<30 \mathrm{GeV}$. This fraction does not show any strong $p_{T}^{J / \psi}$ dependence within the region measured. The measurements are in good agreement with the predictions of NLO NRQCD, but lie slightly below the prediction at high $p_{T}^{J / \psi}\left(p_{T}^{J / \psi}>18 \mathrm{GeV}\right)$. The measurements are also in good agreement with the $\mathrm{LHCb}$ data, measured in the forward region $\left(2.0<y^{J / \psi}<4.5\right)$, where the measurements overlap [82]. Figure 7.33 shows a comparison of measurements of $R_{\chi_{c}}$ made at the LHC, at the Tevatron and at the HERA-B $p A$ fixed target experiment. In general, all of the hadron collider measurements are in good agreement, though the low $p_{T}^{J / \psi}$ data of LHCb and CDF suggest different trends.

\subsubsection{Prompt cross section ratio: $\sigma\left(\chi_{c 2}\right) / \sigma\left(\chi_{c 1}\right)$}

The measurements of the prompt production cross-section of the $\chi_{c 2}$ state, relative to the $\chi_{c 1}$ state, shown in Figure 7.30 , show that the $\chi_{c 1}$ state is more readily produced than the $\chi_{c 2}$ state within the region studied (accounting for their relative branching fractions, a factor of around 1.8). The cross section ratio, measured as a function of $p_{T}^{J / \psi}$, is generally well described by the predictions of NLO NRQCD, though the data suggest an alternative $p_{T}^{J / \psi}$ dependence for $p_{T}^{J / \psi}>18 \mathrm{GeV}$. The prediction of the CSM significantly underestimates the measurements by more than a factor of 2. The measurements are in good agreement with those of CMS, which are performed in a very similar kinematic region [40].

\subsubsection{Non-prompt differential cross sections}

The non-prompt $\chi_{c 1}$ and $\chi_{c 2}$ production cross sections are in general agreement with the theoretical predictions of a model based upon the FONLL approach to b-hadron production, as shown in Figures 7.27 and 7.28, though the measurements 


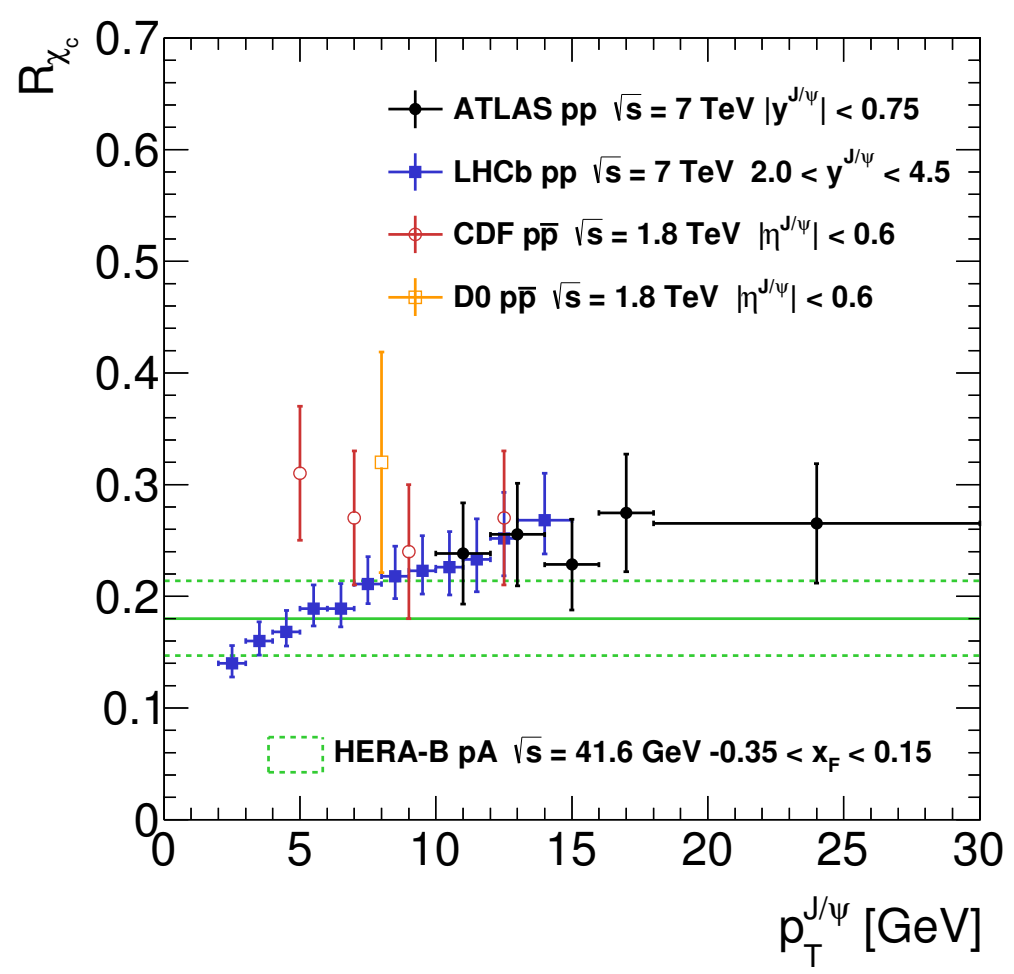

Figure 7.33: A comparison of $R_{\chi_{c}}$ measurements from hadron collider and fixed target experiments. The results presented here are denoted ATLAS and are compared to the measurements of LHCb [82], CDF [54], D0 [70] and HERA-B [160]. $x_{F}$ denotes the Feynman longitudinal momentum fraction.

tend to lie below the predictions at high $p_{T}$. The FONLL based model describes the total non-prompt cross section in terms of $B$-meson production alone, neglecting potential differences in the kinematics and inclusive branching fractions of the contributions from $B_{s}^{0}$ and $b$-baryon decays. The slight overestimation of the nonprompt cross sections by this model may suggest that the inclusive branching fraction $\mathcal{B}\left(H_{b} \rightarrow \chi_{c 1,2} X\right)$ (where $H_{b}$ denotes the relevant mixture of $b$-hadrons produced at the LHC) may not be accurately approximated by the same inclusive branching fraction for the $B$ meson admixture produced in $\Upsilon(4 S)$ decays. However, discrepancies between the predictions of the FONLL approach and experimental data have also been observed in several measurements of high $p_{T}(>20 \mathrm{GeV})$ non-prompt $J / \psi$ and $\psi(2 S)$ production at the LHC [77-79,81]. In the case of the $J / \psi$ and $\psi(2 S)$, the appropriate inclusive branching fractions are available (i.e. the $B^{ \pm, 0}$ meson only ap- 
proximation is not necessary), suggesting that another aspect of the model may be responsible for these discrepancies. One potential source of this discrepancy is the $b$ quark fragmentation functions. The fragmentation functions used in the FONLL approach are fitted from $Z \rightarrow b \bar{b}$ decay data from LEP [56]. However, as discussed in Section 3.2.6, the softer $b$ quark $p_{T}$ spectrum produced at the LHC may not be accurately described by fragmentation functions fitted to the higher $b$-quark energy LEP data.

\subsubsection{Non-prompt cross section ratio: $\sigma\left(\chi_{c 2}\right) / \sigma\left(\chi_{c 1}\right)$}

The measurements of the non-prompt production cross-section of the $\chi_{c 2}$ state, relative to the $\chi_{c 1}$ state, shown in Figure 7.31 as a function of $p_{T}^{J / \psi}$, suggest that the $\chi_{c 1}$ state is produced more readily in $b$-hadron decays than the $\chi_{c 2}$ state (by around a factor of 3 ). The measurements do not exhibit any significant dependence on $p_{T}^{J / \psi}$ in the high $p_{T}^{J / \psi}$ region measured. This is expected given the common production mechanism and the very small mass difference between the two states, relative to the average $p_{T}^{J / \psi}$ being measured. These measurements are in agreement with a single measurement from CDF for $p_{T}^{J / \psi}>10 \mathrm{GeV}$ [38]. The non-prompt production asymmetry for $\chi_{c 1}$ and $\chi_{c 2}$ also provides a measurement of the ratio of branching fractions,

$$
\frac{\mathcal{B}\left(\chi_{c 2} \rightarrow J / \psi \gamma\right)}{\mathcal{B}\left(\chi_{c 1} \rightarrow J / \psi \gamma\right)} \cdot \frac{\mathcal{B}\left(b \rightarrow \chi_{c 2} X\right)}{\mathcal{B}\left(b \rightarrow \chi_{c 1} X\right)}=0.19 \pm 0.04
$$

where $b$ denotes the mixture of $b$-hadron species produced at the LHC. This measurement is obtained by fitting the data in Figure 7.31 with the ratio of the $\chi_{c 2}$ and $\chi_{c 1}$ curves for the FONLL based predictions shown in Figure 7.28 (the theoretically

predicted ratio has a constant value of 0.97 across the range of $p_{T}^{J / \psi}$ studied). The corresponding value calculated from the world average values for the inclusive $B$ meson branching fractions $\mathcal{B}\left(B \rightarrow \chi_{c 1,2} X\right)$ (used due to an absence of data for the 
b-hadron mixture relevant to the LHC) is $0.19 \pm 0.06$ [3], while LHCb report a value of $0.184 \pm 0.025$ (stat.) \pm 0.015 (syst.) [149], both consistent with this measurement.

\subsubsection{Non-prompt fractions}

The fractions of $\chi_{c 1}$ and $\chi_{c 2}$ produced in b-hadron decays, shown in Figure 7.32, tend to increase slightly as a function of $p_{T}^{\chi_{c}}$. This behaviour is also observed for inclusive $J / \psi$ and $\psi(2 S)$ production $[77,78]$. However, contrary to the situation for $J / \psi$ and $\psi(2 S)$, the inclusive production of $\chi_{c 1}$ and $\chi_{c 2}$ is dominated by prompt production for $p_{T}>20 \mathrm{GeV}$. This small non-prompt fraction is also observed in $\sqrt{s}=1.96 \mathrm{TeV} p \bar{p}$ collisions, from which CDF measured around $(15 \pm 1) \%$ of $\chi_{c 1}$ and $(6 \pm 1) \%$ of $\chi_{c 2}$ to be produced in $b$-hadron decays for $p_{T}^{J / \psi}>4 \mathrm{GeV}$ (assuming an equal total efficiency for prompt and non-prompt $\left.\chi_{c J}\right)$ [38]. An earlier measurement from CDF quotes a combined $\chi_{c 1}$ and $\chi_{c 2}$ non-prompt fraction of $(10.8 \pm 3.0) \%$ for $p_{T}^{J / \psi}>4 \mathrm{GeV}[54]$.

\subsubsection{Summary}

In general, the predictions of NLO NRQCD consistently provide a good description of the prompt $\chi_{c 1}$ and $\chi_{c 2}$ measurements. The same predictions also provide a similarly good description of the prompt production of $J / \psi$ and $\psi(2 S)[78,79,81]$. The production of the $\chi_{c 1}$ and $\chi_{c 2}$ states in $b$-hadron decays is generally well described by the predictions of the FONLL approach. However, the large experimental uncertainties and approximations used to described the relevant inclusive branching fractions make it difficult to draw any firm conclusions on the reliability of the FONLL approach from these measurements. 


\subsection{Supporting Measurements}

This section is devoted to demonstrating the validity and accuracy of the various efficiency corrections used to perform the main $\chi_{c}$ analysis through two supporting measurements. The first measurement, a measurement of $J / \psi$ production cross sections, is designed to validate the efficiency corrections associated with the $J / \psi \rightarrow$ $\mu^{+} \mu^{-}$decay while the second measurement, a measurement of the branching fraction $\mathcal{B}\left(B^{ \pm} \rightarrow \chi_{c 1} K^{ \pm}\right)$, is designed validate the efficiency corrections associated with the $\chi_{c J} \rightarrow J / \psi \gamma$ decay, specifically the conversion reconstruction efficiency.

\subsubsection{Measurements of $J / \psi$ production}

A measurement of the cross sections for the inclusive, prompt and non-prompt production of the $J / \psi$ charmonium state in $\sqrt{s}=7 \mathrm{TeV} p p$ collisions is performed with a subset of the data sample described in Section 7.3. The measurement makes use of the same muon reconstruction and di-muon trigger efficiency corrections used in the measurements of $\chi_{c 1}$ and $\chi_{c 2}$ production in addition to an identical $J / \psi \rightarrow \mu^{+} \mu^{-}$ selection and a very similar data-fitting procedure (described in Section 7.6). The aim of this study is to validate these elements of the $\chi_{c}$ analysis by comparing these measurements of the $J / \psi$ production cross sections with published ATLAS measurements performed using independent data samples, efficiency corrections and event selections [77]. The measurements are performed in a kinematic region, $\left|y^{J / \psi}\right|<0.75$

and $p_{T}^{J / \psi}>8 \mathrm{GeV}$, which includes the region used in the $\chi_{c}$ analysis $\left(\left|y^{J / \psi}\right|<0.75\right.$ and $\left.10<p_{T}^{J / \psi}<30 \mathrm{GeV}\right)$. 


\subsubsection{Data Sample and Event Selection}

The measurement is performed with a sample of $\sqrt{s}=7 \mathrm{TeV} p p$ collision data, representing an integrated luminosity of $157 \mathrm{pb}^{-1}$, collected towards the beginning of the 2011 LHC run. The data sample was collected with the EF_2mu4_Jpsimumu di-muon trigger described in Section 7.3. $J / \psi \rightarrow \mu^{+} \mu^{-}$decays are selected with the criteria described in Section 7.4.1 (the di-muon invariant mass cut is not applied).

\subsubsection{Cross Section Measurement Procedure}

The double differential cross section for $J / \psi$ production is given by,

$$
\frac{d^{2} \sigma(J / \psi)}{d p_{T} d y} \times \mathcal{B}\left(J / \psi \rightarrow \mu^{+} \mu^{-}\right)=\frac{N_{J / \psi}}{\mathcal{L} \times \Delta p_{T} \times \Delta y}
$$

where $N_{J / \psi}$ is the $J / \psi$ yield in a bin of $J / \psi$ transverse momentum and rapidity, corrected for experimental losses (efficiency and acceptance), $\mathcal{L}$ is the integrated luminosity of the data sample and $\Delta p_{T}$ and $\Delta y$ are bin widths in $J / \psi$ transverse momentum and rapidity, respectively. As in the $\chi_{c}$ analysis, each reconstructed $J / \psi \rightarrow \mu^{+} \mu^{-}$candidate is given a weight to account for experimental losses due to finite acceptance and reconstruction and trigger efficiencies. The per-candidate weight, $w$, is given by,

$$
w^{-1}=\mathcal{A} \cdot \epsilon_{\text {trig }} \cdot \epsilon_{\text {di-muon }}
$$

where $\mathcal{A}$ is the per-candidate acceptance and $\epsilon_{\text {trig }}$ and $\epsilon_{\text {di-muon }}$ are the di-muon trigger and reconstruction efficiencies, respectively. The trigger efficiency, $\epsilon_{\text {trig }}$, is identical that used in the $\chi_{c}$ analysis and is described in Section 7.5.2. The $J / \psi \rightarrow \mu^{+} \mu^{-}$ reconstruction efficiency, $\epsilon_{\mathrm{di}-\mathrm{muon}}$, is also identical to that used in the $\chi_{c}$ analysis and is described in Section 7.5.3 but with the term $\epsilon_{\text {mass }}$ (the correction for the di-muon invariant mass cut) removed. The acceptance correction, $\mathcal{A}$, is calculated using the 
same MC approach used in the $\chi_{c}$ analysis, described in Section 7.5.1, to correct for the fiducial cuts $p_{T}^{\mu}>4 \mathrm{GeV}$ and $\left|\eta^{\mu}\right|<2.3$. The acceptance simulation assumes isotropic angular distributions for the $J / \psi \rightarrow \mu^{+} \mu^{-}$decays, the same assumption made in the ATLAS measurement, which is used as a reference [77]. The acceptance correction is parametrised as a map binned in $p_{T}^{J / \psi}$ and $\left|y^{J / \psi}\right|$, as shown in Figure 7.34.

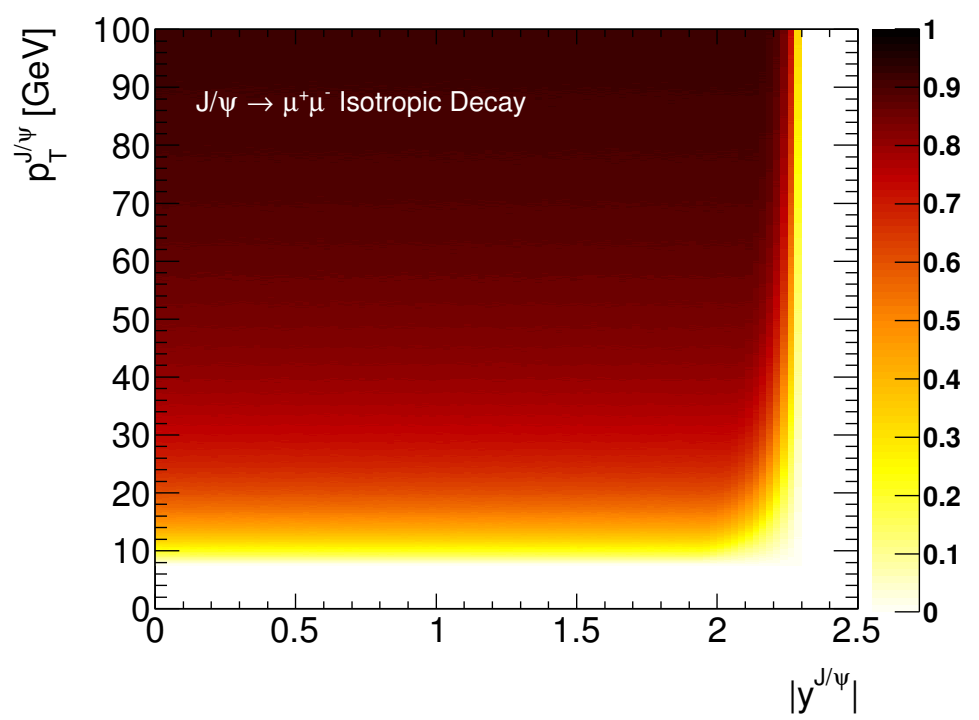

Figure 7.34: Acceptance map for $J / \psi \rightarrow \mu^{+} \mu^{-}$decays binned in $p_{T}^{J / \psi}$ and $\left|y^{J / \psi}\right|$, to correct for the fiducial cuts $p_{T}^{\mu}>4 \mathrm{GeV}$ and $\left|\eta^{\mu}\right|<2.3$.

Corrected yields, $N_{J / \psi}$, are measured in bins of $p_{T}^{J / \psi}$ with an unbinned maximum likelihood fit to the weighted di-muon invariant mass, $m_{\mu^{+} \mu^{-}}$, and pseudoproper decay time, $\tau$, distributions of the selected $J / \psi \rightarrow \mu^{+} \mu^{-}$candidates. The 2-dimensional fit is conceptually similar to that described in Section 5.6.2, and allows the prompt and non-prompt contributions to $J / \psi$ production to be separated. The technical implementation of the fitting procedure is very similar to that used in the fit performed in the $\chi_{c}$ analysis, described in Section 7.6. The fits are performed within the region $2.75<m_{\mu^{+} \mu^{-}}<3.45 \mathrm{GeV}$, which includes the full $J / \psi$ peak but excludes the $\psi(2 S)$ region of the di-muon invariant mass spectrum. The PDF used to perform the fit takes the form, 


$$
F\left(m_{\mu^{+} \mu^{-}}, \tau, \delta \tau\right)=f_{\text {sig }} \cdot F_{\text {sig }}\left(m_{\mu^{+} \mu^{-}}, \tau, \delta \tau\right)+\left(1-f_{\text {sig }}\right) \cdot F_{\text {bkgd }}\left(m_{\mu^{+} \mu^{-}}, \tau, \delta \tau\right),
$$

where $f_{\text {sig }}$ is the fraction of $J / \psi$ signal events, $F_{\text {sig }}$ is the $J / \psi$ signal PDF, and $F_{\text {bkgd }}$ is the background PDF and $\delta \tau$ is the per-candidate uncertainty on the pseudo-proper decay time $\tau$. Both $F_{\text {sig }}$ and $F_{\text {bkgd }}$ are represented by a sum of products between PDFs describing the prompt (P) and non-prompt (NP) contributions to the $m_{\mu^{+} \mu^{-}}$ and $\tau$ projections of the 2-dimensional $m_{\mu^{+} \mu^{-}} \tau$ distributions,

$$
\begin{aligned}
F_{\mathrm{sig}(\mathrm{bkgd})} & =\left(1-f_{\mathrm{sig}(\mathrm{bkgd})}^{\mathrm{NP}}\right) \cdot M_{\mathrm{sig}(\mathrm{bkgd})}^{\mathrm{P}}\left(m_{\mu^{+} \mu^{-}}\right) \cdot T_{\mathrm{sig}(\mathrm{bkgd})}^{\mathrm{P}}(\tau, \delta \tau) \\
& +f_{\mathrm{sig}(\mathrm{bkgd})}^{\mathrm{NP}} \cdot M_{\mathrm{sig}(\mathrm{bkgd})}^{\mathrm{NP}}\left(m_{\mu^{+} \mu^{-}}\right) \cdot T_{\mathrm{sig}(\mathrm{bkgd})}^{\mathrm{NP}}(\tau, \delta \tau) .
\end{aligned}
$$

The fraction $f_{\mathrm{sig}(\mathrm{kkgd})}^{\mathrm{NP}}$ denotes the fraction of non-prompt signal (background) candidates. Both the prompt and non-prompt $m_{\mu^{+} \mu^{-}}$projections of the $J / \psi$ signal PDF, $M_{\text {sig }}^{(\mathrm{N}) \mathrm{P}}$, are described by a Novosibirsk function [122]. The Novosibirsk function has four free parameters, which describe the mean, the width and the extents of the left and right tails. Both the mean and width parameters are freely determined by the fit. No significant variations in the $J / \psi \rightarrow \mu^{+} \mu^{-}$line-shape are observed in different $p_{T}^{J / \psi}$ bins and the two parameters describing the shapes of the tails are fixed to values determined in a fit to the $m_{\mu^{+} \mu^{-}}$distribution of the full data sample, $p_{T}^{J / \psi}>8 \mathrm{GeV}$. The prompt and non-prompt $J / \psi$ signal components share common mean and width parameters. The prompt and non-prompt $m_{\mu^{+} \mu^{-}}$projections of the $J / \psi$ background PDF, $M_{\mathrm{bkgd}}^{(\mathrm{N}) \mathrm{P}}$, are described by exponential functions. The prompt and non-prompt background components each have an independent free parameter describing the slope of the background distribution. The $\tau$ projections of the prompt and non-prompt components of the signal and background PDFs, $T_{\mathrm{sig}(\mathrm{bkgd})}^{(\mathrm{N}) \mathrm{P}}$, are identical to those used in the $\chi_{c}$ analysis, described in Section 7.6. In summary, 
the prompt (signal and background) components are described by delta functions, while the non-prompt components are described by exponential functions. Each component of the $\tau$ projections of the PDF is convolved with a resolution function determined from the per-candidate uncertainty, $\delta \tau$, on the pseudo-proper decay time $\tau$, as described in Section 7.6. Figures 7.35, 7.36 and 7.37 show the full set of fits performed in bins of $p_{T}^{J / \psi}$.

\subsubsection{Systematic Uncertainties}

Given that the aim of this study is to validate the efficiency corrections and analysis procedures of the $\chi_{c}$ measurement, a detailed study into potential systematic uncertainties is not performed. However, a representative systematic uncertainty associated with several aspects of this analysis can be estimated from the in-depth studies performed in the $\chi_{c}$ analysis (described in Section 7.8). In particular, $p_{T}^{J / \psi}$ independent systematic uncertainties of $\pm 1 \%, \pm 1 \%$ and $\pm 4 \%$ are estimated for the ID track, muon identification and trigger efficiency corrections respectively, based on the studies discussed in Section 7.8. The uncertainty in the measurement of the integrated luminosity is estimated to be $\pm 1.8 \%$ [110]. From these estimates, a $p_{T}^{J / \psi}$ independent total systematic uncertainty on these measurements of $4.6 \%$ is found. The statistical uncertainties on the measurements range from $2 \%$ to $4 \%$, depending on the $p_{T}^{J / \psi}$ bin. 

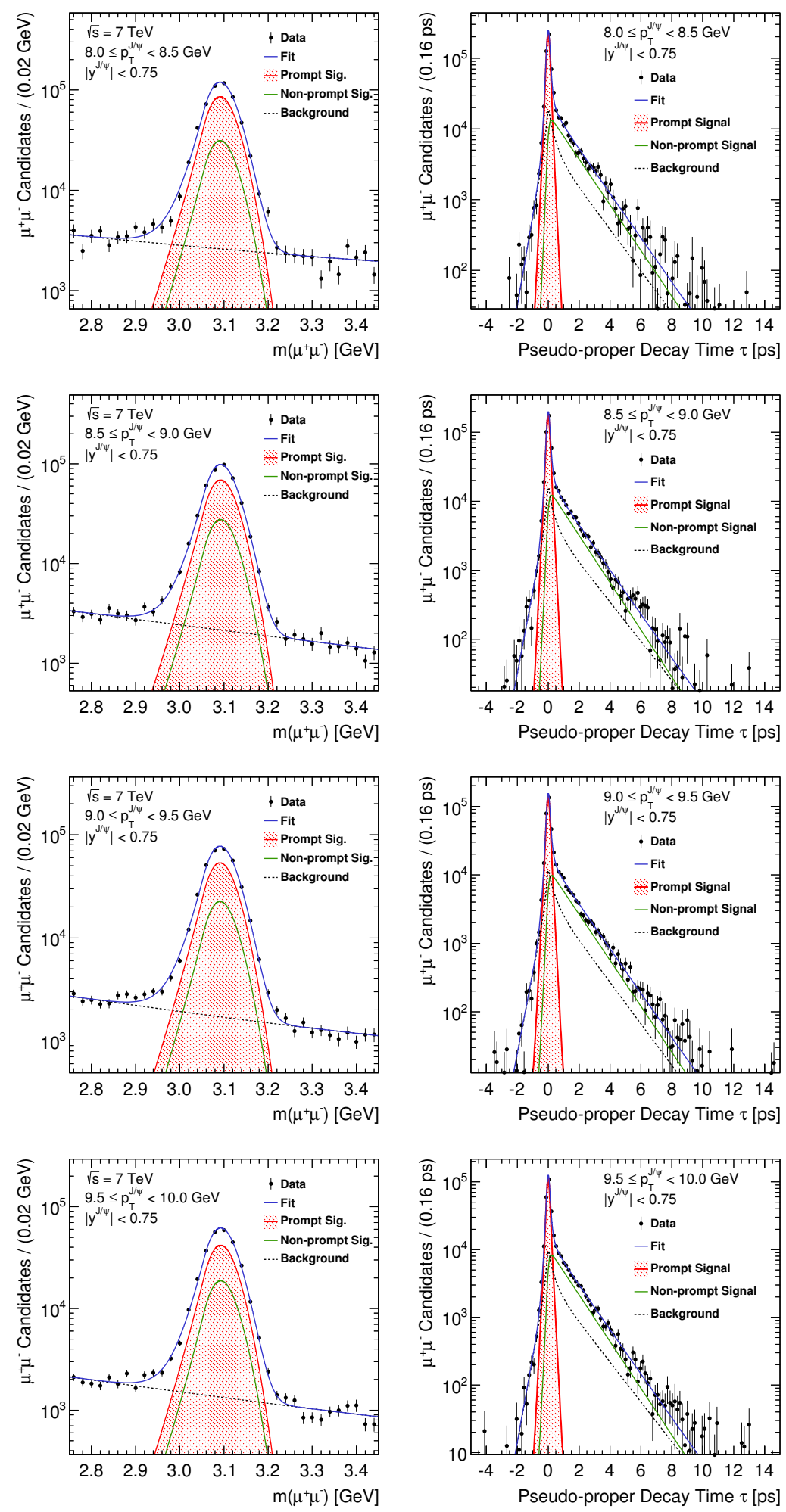

Figure 7.35: Simultaneous fits to the di-muon invariant mass, $m\left(\mu^{+} \mu^{-}\right)$, and pseudoproper decay time, $\tau$, distributions of $J / \psi \rightarrow \mu^{+} \mu^{-}$candidates in four bins of $p_{T}^{J / \psi}$ within $8<p_{T}^{J / \psi}<10 \mathrm{GeV}$. 

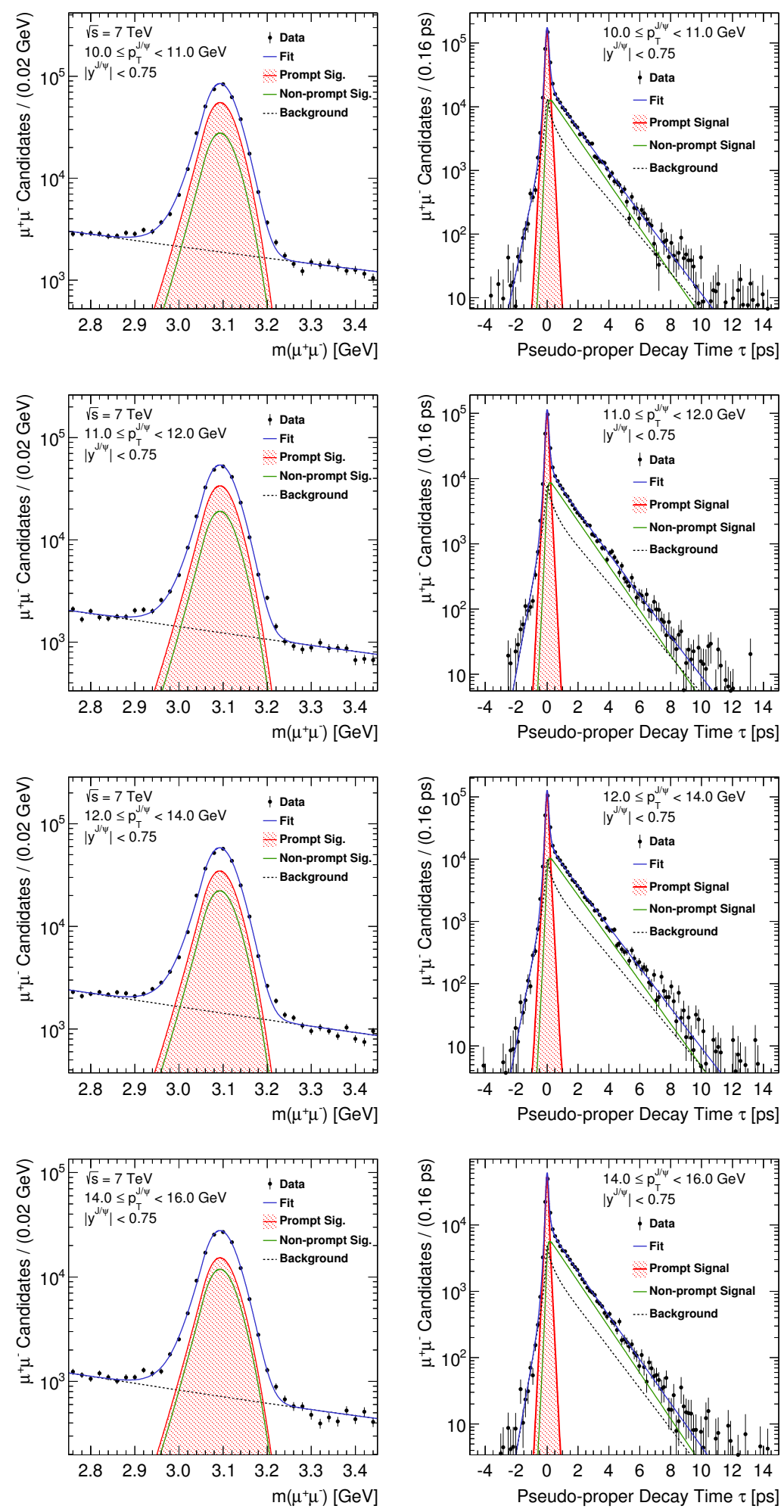

Figure 7.36: Simultaneous fits to the di-muon invariant mass, $m\left(\mu^{+} \mu^{-}\right)$, and pseudoproper decay time, $\tau$, distributions of $J / \psi \rightarrow \mu^{+} \mu^{-}$candidates in four bins of $p_{T}^{J / \psi}$ within $10<p_{T}^{J / \psi}<16 \mathrm{GeV}$. 

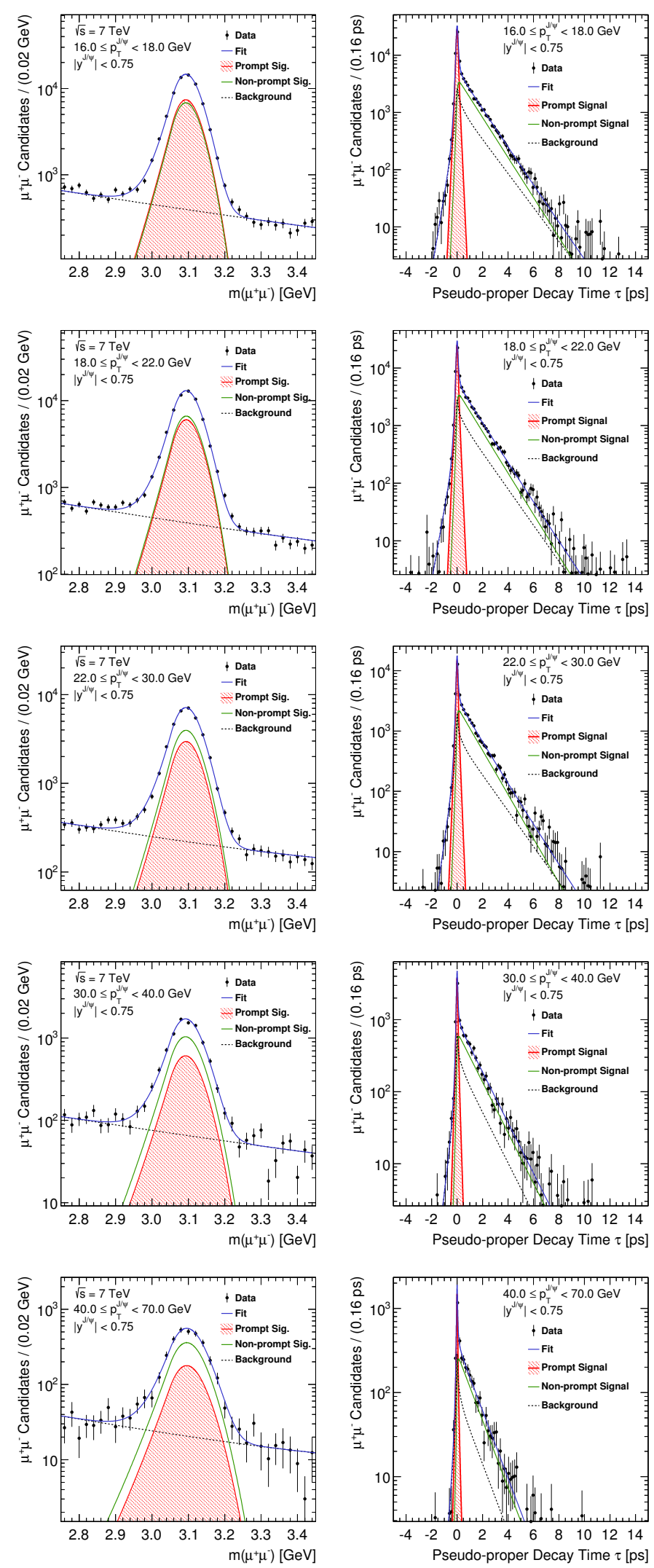

Figure 7.37: Simultaneous fits to the di-muon invariant mass, $m\left(\mu^{+} \mu^{-}\right)$, and pseudoproper decay time, $\tau$, distributions of $J / \psi \rightarrow \mu^{+} \mu^{-}$candidates in five bins of $p_{T}^{J / \psi}$ within $16<p_{T}^{J / \psi}<70 \mathrm{GeV}$. 


\subsubsection{Results and Conclusion}

The measured differential cross section for inclusive $J / \psi$ production is shown in Figure 7.38; the differential cross sections for prompt and non-prompt $J / \psi$ production are shown in Figure 7.39 and the non-prompt fraction of inclusive $J / \psi$ production is shown in Figure 7.40, all measured as functions of $p_{T}^{J / \psi}$. All of these measurements are compared to the published ATLAS measurements, which are used as a reference [77]. The measurements from this analysis are in good agreement with the reference measurements. This suggests that the combined analysis procedure, used to select, fit and correct $J / \psi \rightarrow \mu^{+} \mu^{-}$candidates, is accurate to at least the level of the total uncertainty on the reference measurements, which is around $10 \%$. 

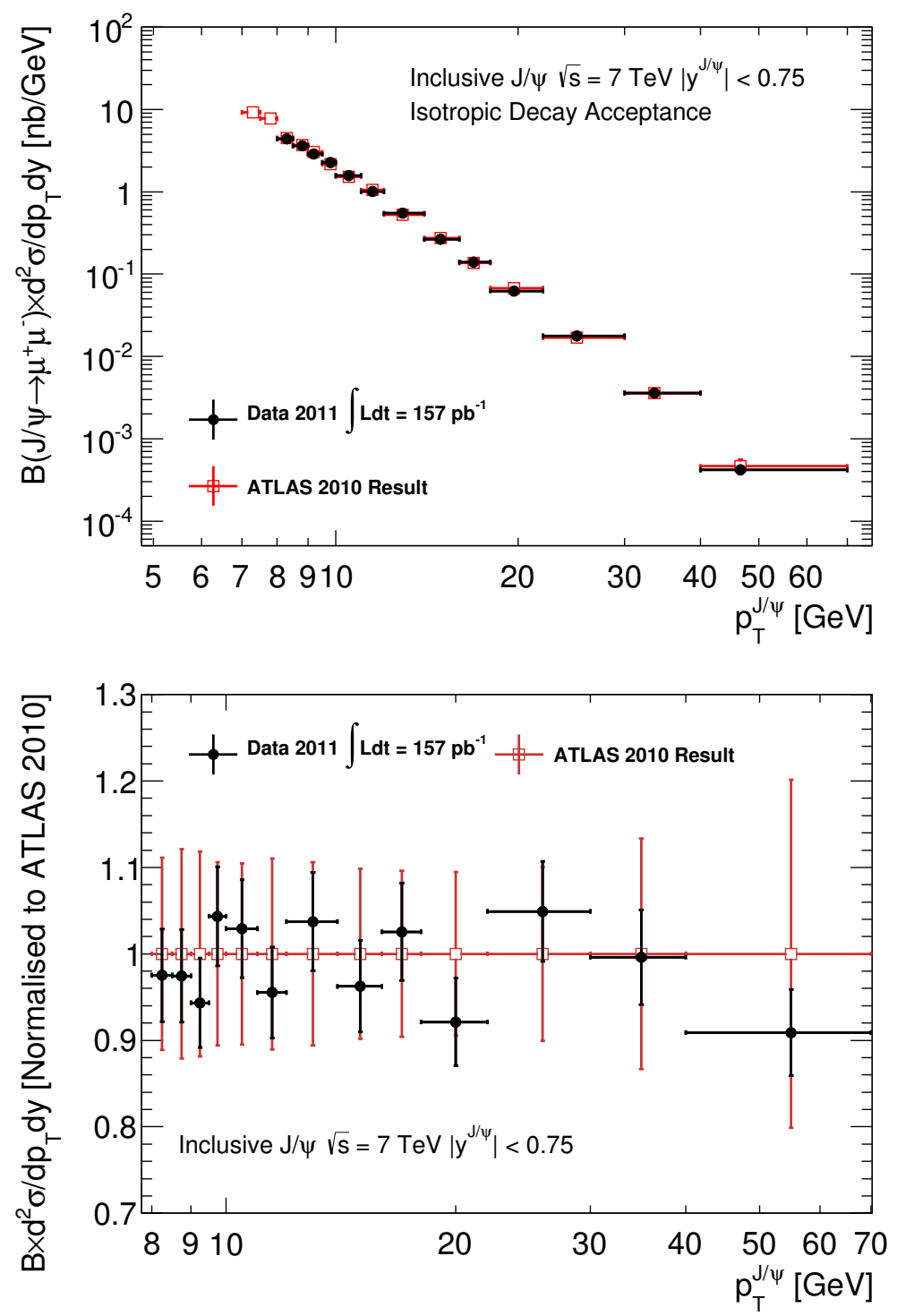

Figure 7.38: The differential cross section for inclusive $J / \psi$ production in $p p$ collisions at $\sqrt{s}=7 \mathrm{TeV}$. The black points shown the measurements of this analysis, while the red points show the reference measurements from [77]. The lower figure shows the ratio of the measurements with respect to the published ATLAS results, $B=\left(J / \psi \rightarrow \mu^{+} \mu^{-}\right)$. 

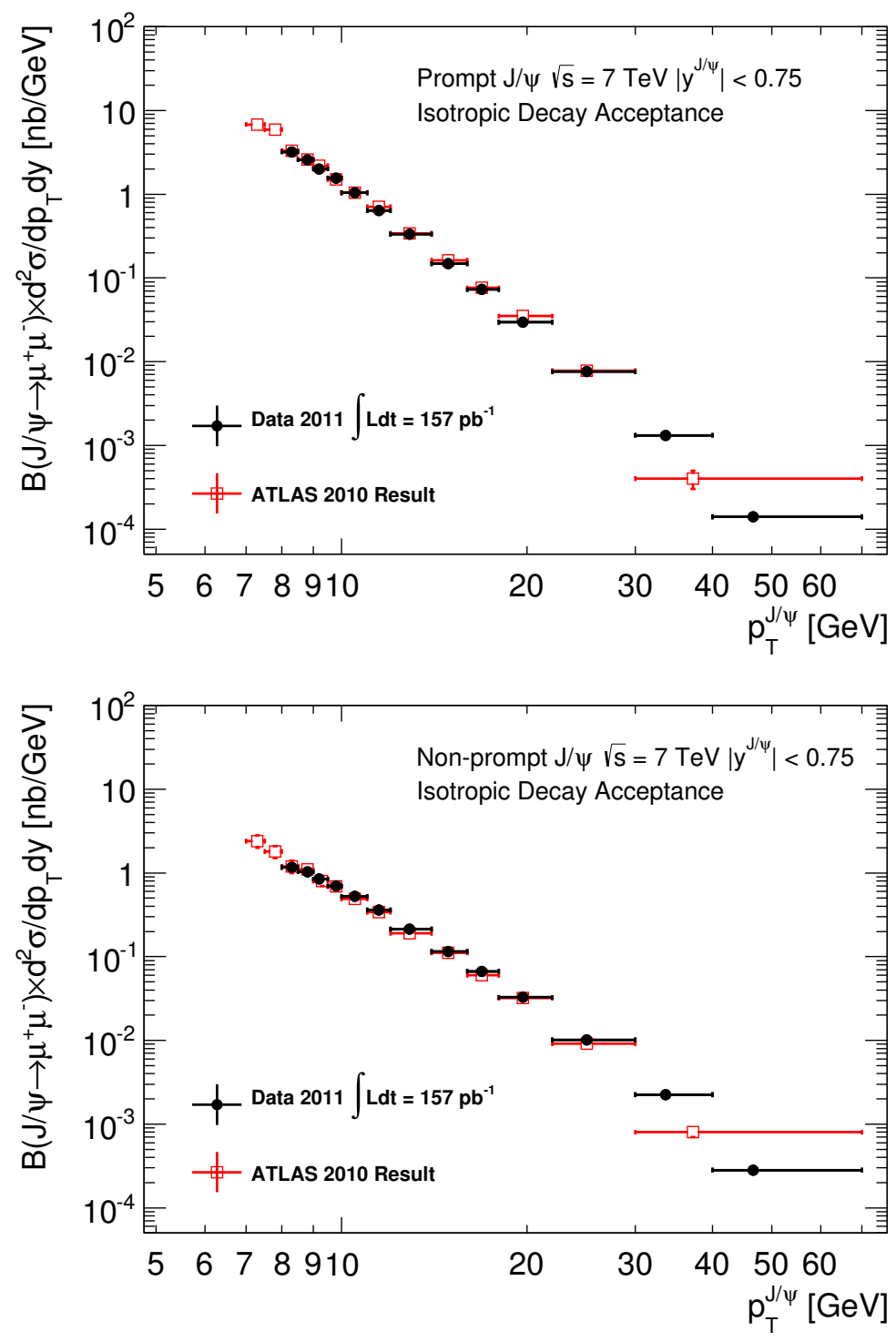

Figure 7.39: The differential cross section for prompt (top) and non-prompt (bottom) $J / \psi$ production in $p p$ collisions at $\sqrt{s}=7 \mathrm{TeV}$. The black points show the measurements of this analysis, while the red points show the reference measurements from [77]. 


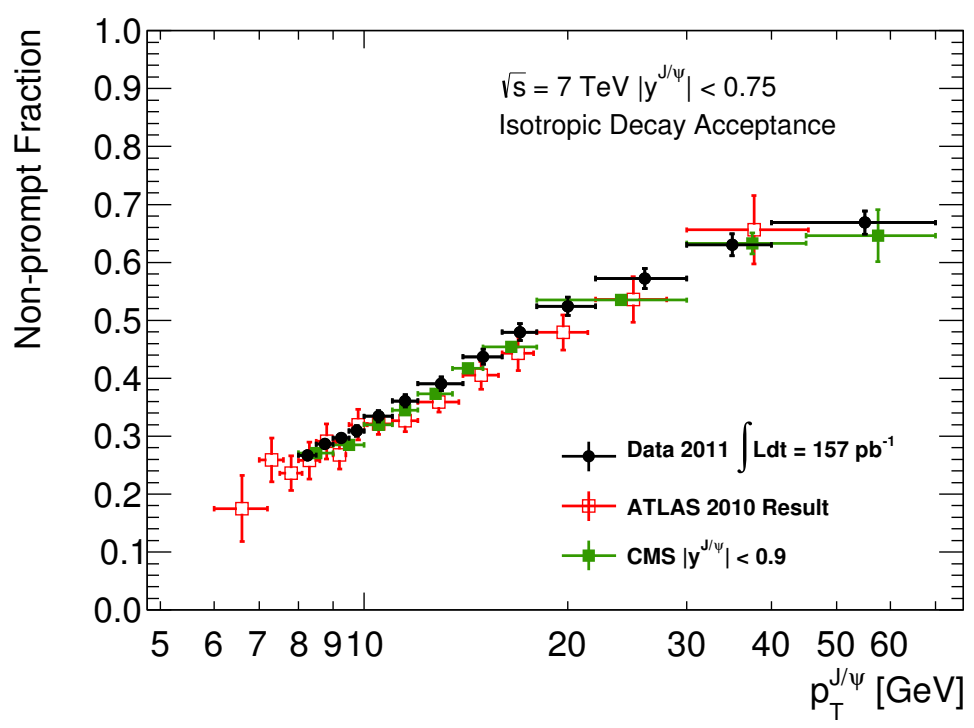

Figure 7.40: The fraction of $J / \psi$ produced in the decay of $b$-hadrons, $f_{\text {non-prompt }}$, measured as a function of $p_{T}^{J / \psi}$, in $p p$ collisions at $\sqrt{s}=7 \mathrm{TeV}$. The black points show the measurements of this analysis, while the red points show the reference measurements from [77]. The measurements of CMS, within the region $\left|y^{J / \psi}\right|<0.9$, are also shown by the green data points [78]. 


\subsubsection{Measurement of $\mathcal{B}\left(B^{ \pm} \rightarrow \chi_{c 1} K^{ \pm}\right)$}

The branching fraction for the decay $B^{ \pm} \rightarrow \chi_{c 1} K^{ \pm}$is measured using the same dataset, event selection and reconstruction efficiencies used in the main $\chi_{c}$ analysis described in Chapter 7. The aim of this measurement is to validate the conversion reconstruction efficiency, derived from MC simulation as described in Section 7.5.4, through a comparison of the measured value of this branching fraction with the current world average measurement. Several measurements of $\mathcal{B}\left(B^{ \pm} \rightarrow \chi_{c 1} K^{ \pm}\right)$ have been made at various $e^{+} e^{-}$and hadron collider experiments. The current world average value, $\mathcal{B}\left(B^{ \pm} \rightarrow \chi_{c 1} K^{ \pm}\right)=(4.79 \pm 0.26) \times 10^{-4}[3]$, is dominated by the measurements of Belle [161] and BaBar [162].

The relative production rates of the decays $B^{ \pm} \rightarrow \chi_{c 1} K^{ \pm}$and $B^{ \pm} \rightarrow J / \psi K^{ \pm}$ (with $\chi_{c 1} \rightarrow J / \psi \gamma$ and $J / \psi \rightarrow \mu^{+} \mu^{-}$in both decays) can be used to measure $\mathcal{B}\left(B^{ \pm} \rightarrow \chi_{c 1} K^{ \pm}\right)$, with knowledge of the branching fractions $\mathcal{B}\left(B^{ \pm} \rightarrow J / \psi K^{ \pm}\right)$and $\mathcal{B}\left(\chi_{c 1} \rightarrow J / \psi \gamma\right)$. The use of $B^{ \pm} \rightarrow J / \psi K^{ \pm}$as a reference channel is motivated by the fact that it has a similar final state to the $B^{ \pm} \rightarrow \chi_{c 1} K^{ \pm} \rightarrow J / \psi \gamma K^{ \pm}$decay (with similar kinematics) and the branching fraction $\mathcal{B}\left(B^{ \pm} \rightarrow J / \psi K^{ \pm}\right)$has been precisely measured, $\mathcal{B}\left(B^{ \pm} \rightarrow J / \psi K^{ \pm}\right)=(1.016 \pm 0.033) \times 10^{-3}$ [3]. The branching fraction $\mathcal{B}\left(\chi_{c 1} \rightarrow J / \psi \gamma\right)=0.344 \pm 0.015$ is also known to a good precision [3]. Assuming equal production rates for the $B^{+}$and $B^{-}$states in $p p$ collisions (an assumption likely to be accurately fulfilled, given that $b \bar{b}$ production represents the dominant $B^{ \pm}$production mechanism in $p p$ collisions), the number of reconstructed $B^{ \pm} \rightarrow \chi_{c 1} K^{ \pm}$and $B^{ \pm} \rightarrow J / \psi K^{ \pm}$decays (within the same data sample) can be related to $\mathcal{B}\left(B^{ \pm} \rightarrow \chi_{c 1} K^{ \pm}\right)$as,

$$
\mathcal{B}\left(B^{ \pm} \rightarrow \chi_{c 1} K^{ \pm}\right)=\mathcal{A}_{B} \cdot \frac{N_{\chi_{c 1}}^{B}}{N_{J / \psi}^{B}} \cdot \frac{\mathcal{B}\left(B^{ \pm} \rightarrow J / \psi K^{ \pm}\right)}{\mathcal{B}\left(\chi_{c 1} \rightarrow J / \psi \gamma\right)}
$$

where $N_{\chi_{c 1}}^{B}$ and $N_{J / \psi}^{B}$ are the efficiency-corrected reconstructed yields of $B^{ \pm} \rightarrow$ $\chi_{c 1} K^{ \pm}$and $B^{ \pm} \rightarrow J / \psi K^{ \pm}$decays, respectively. $\mathcal{A}_{B}$ is a correction factor to account 
for the differing acceptances of the two decays.

$B^{ \pm} \rightarrow \chi_{c 1} K^{ \pm}$and $B^{ \pm} \rightarrow J / \psi K^{ \pm}$decays are reconstructed within the same data sample, representing an integrated luminosity of $4.5 \mathrm{fb}^{-1}$ of $\sqrt{s}=7 \mathrm{TeV} p p$ collision data, used to perform the $\chi_{c}$ analysis (see Section 7.3). The $J / \psi$ fiducial region $\left(10<p_{T}^{J / \psi}<30 \mathrm{GeV}\right.$ and $\left.\left|y^{J / \psi}\right|<0.75\right)$ and $\chi_{c}$ selection are kept identical to those used in the main $\chi_{c}$ analysis to maximise the validity of the cross-check.

\subsubsection{Selection of candidate $B^{ \pm} \rightarrow J / \psi K^{ \pm}$and $B^{ \pm} \rightarrow \chi_{c 1} K^{ \pm}$ decays}

The selection of $B^{ \pm} \rightarrow J / \psi K^{ \pm}$and $B^{ \pm} \rightarrow \chi_{c 1} K^{ \pm}$decays is seeded by the selection of $J / \psi$ and $\chi_{c}$ candidates within events firing the EF_2mu4(T)_Jpsimumu triggers with the same procedure described as in Section 7.4. Tracks are selected within these events which satisfy the following criteria:

- Each track must contain at least one hit in the pixel layers.

- Each track must contain at least six hits in the SCT layers.

- The transverse momentum of the track must satisfy $p_{T}^{K}>3.0 \mathrm{GeV}$.

- The pseudorapidity of the track must be within $\left|\eta^{K}\right|<2.5$.

Tracks which satisfy these requirements are assigned the charged kaon mass and are considered as candidate charged kaons. The ATLAS detector has no reliable particle identification capabilities for tracks with $p_{T}>3.0 \mathrm{GeV}$. These tracks are grouped with the tracks associated with the $J / \psi \rightarrow \mu^{+} \mu^{-}$candidate and a three-track vertex fit is performed to determine the $\mu^{+} \mu^{-} K^{ \pm}$vertex. These $J / \psi K^{ \pm}$ candidates are retained for further analysis if the fit quality of this vertex satisfies $\chi^{2} /[$ d.o.f. $]<6$. An additional requirement of $L_{x y}>0.3 \mathrm{~mm}$ (defined in Equation 5.3) is imposed to reject promptly produced $J / \psi K^{ \pm}$combinations. Candidate $B^{ \pm} \rightarrow \chi_{c 1} K^{ \pm}$decays are identified from $J / \psi K^{ \pm}$candidates associated with 
a converted photon (i.e. passing the $\chi_{c}$ selection described in Section 7.4). These combinations are required to satisfy $4.65<m\left(\mu^{+} \mu^{-} K^{ \pm}\right)-m\left(\mu^{+} \mu^{-}\right)+m_{J / \psi}<$ $5.2 \mathrm{GeV}$ (where $m_{J / \psi}$ is the world average $J / \psi$ mass [3]) to reject backgrounds from $B^{ \pm} \rightarrow J / \psi K^{ \pm}$decays. The $m\left(\mu^{+} \mu^{-} \gamma\right)-m\left(\mu^{+} \mu^{-}\right)$of selected $B^{ \pm} \rightarrow \chi_{c 1} K^{ \pm}$candidates is shown in Figure 7.41 , the $B^{ \pm} \rightarrow \chi_{c 1} K^{ \pm}$candidates are required to satisfy $0.32<m\left(\mu^{+} \mu^{-} \gamma\right)-m\left(\mu^{+} \mu^{-}\right)<0.43 \mathrm{GeV}$ to select $\chi_{c 1}$ decays.

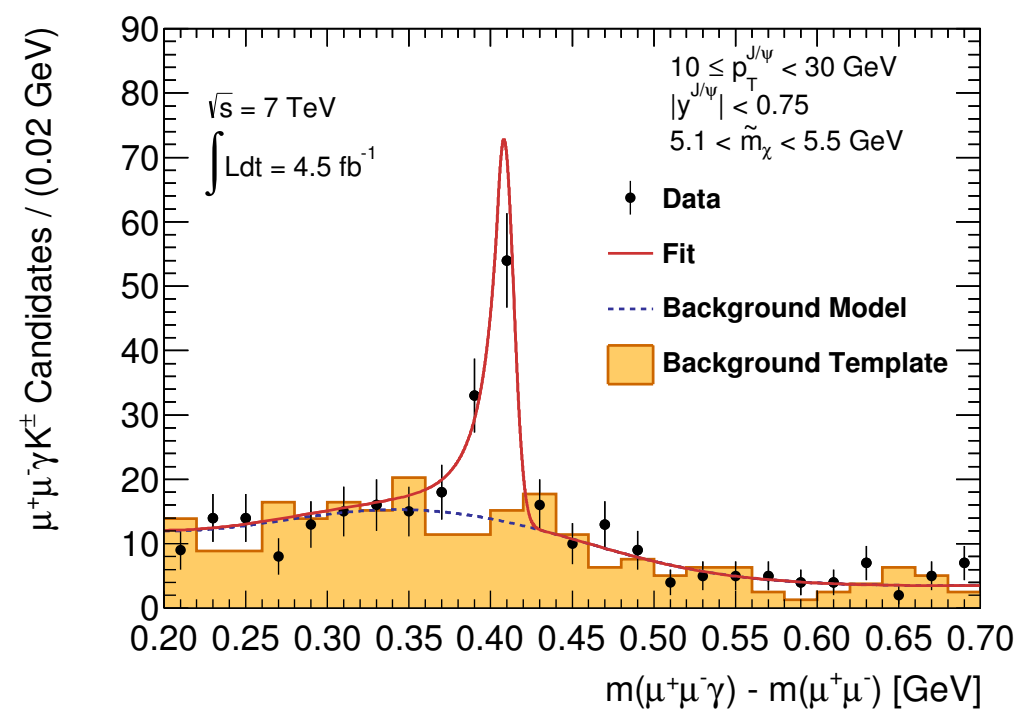

Figure 7.41: The $m\left(\mu^{+} \mu^{-} \gamma\right)-m\left(\mu^{+} \mu^{-}\right)$distribution of selected $B^{ \pm} \rightarrow \chi_{c 1} K^{ \pm}$candidates within $5.1<\tilde{m} \chi_{c 1}<5.5 \mathrm{GeV}$, the region around the $B^{ \pm}$peak $\left(\tilde{m} \chi_{c 1}=\right.$ $\left.m\left(\mu^{+} \mu^{-} \gamma K^{ \pm}\right)-m\left(\mu^{+} \mu^{-} \gamma\right)+m \chi_{c 1}\right)$.

\subsubsection{Calculation of the acceptance correction $\mathcal{A}_{B}$}

The acceptance correction $\mathcal{A}_{B}$ accounts for the difference between the yields of $B^{ \pm} \rightarrow \chi_{c 1} K^{ \pm}$and $B^{ \pm} \rightarrow J / \psi K^{ \pm}$decays due to the differing kinematic acceptances of the two decays. The primary source of this difference is the presence of the photon in the $B^{ \pm} \rightarrow \chi_{c 1} K^{ \pm}$final state, though the differing kinematics of the $\mu^{+} \mu^{-} K^{ \pm}$also contribute to a lesser extent. The correction is calculated with a generator level MC simulation that uses the $B^{ \pm}$production cross section measured by ATLAS in $\sqrt{s}=7 \mathrm{TeV} p p$ collisions as an input [163]. The $B^{ \pm}$production cross sections, measured within three rapidity intervals, are fitted with an analytical function, as 
shown in Figure 7.42. Each set of data points is fitted with the function defined in Equation 7.14 (Section 7.7), containing five free parameters.

The simulation generates $B^{ \pm}$mesons according to the fitted differential cross sections and simulates decays to both $B^{ \pm} \rightarrow \chi_{c 1} K^{ \pm}$and $B^{ \pm} \rightarrow J / \psi K^{ \pm}$. To calculate $\mathcal{A}_{B}$, the simulation counts the number of $B^{ \pm} \rightarrow \chi_{c 1} K^{ \pm}$decays, relative to the number of $B^{ \pm} \rightarrow J / \psi K^{ \pm}$decays, that fall within the fiducial region, defined for both decays as:

- $10<p_{T}^{J / \psi}<30 \mathrm{GeV}$ and $\left|y^{J / \psi}\right|<0.75$,

- $p_{T}^{\mu}>4 \mathrm{GeV}$ and $\left|\eta^{\mu}\right|<2.3$,

- $p_{T}^{K}>3 \mathrm{GeV}$ and $\left|\eta^{K}\right|<2.5$,

- $p_{T}^{\gamma}>1.5 \mathrm{GeV}$ and $\left|\eta^{\gamma}\right|<2.0$ (for $B^{ \pm} \rightarrow \chi_{c 1} K^{ \pm}$only),

- $L_{x y}>0.3 \mathrm{~mm}$.

The charmonium states in $B^{ \pm} \rightarrow \chi_{c 1} K^{ \pm}$and $B^{ \pm} \rightarrow J / \psi K^{ \pm}$decays are produced in a $J_{z}=0$ state (where $z$ is the direction of the $K^{ \pm}$in the $B^{ \pm}$rest frame) to conserve angular momentum (since both the $B^{ \pm}$and $K^{ \pm}$mesons have total angular momentum $J=0$ ). Each simulated decay is weighted such that the angular distributions of the $J / \psi \rightarrow \mu^{+} \mu^{-}$and $\chi_{c 1} \rightarrow J / \psi \gamma \rightarrow \mu^{+} \mu^{-} \gamma$ decays follow the distributions expected for $J / \psi$ and $\chi_{c 1}$ states produced in a $J_{z}=0$ state (longitudinally polarised). The $J / \psi \rightarrow \mu^{+} \mu^{-}$angular distribution for the $B^{ \pm} \rightarrow J / \psi K^{ \pm}$ decay is weighted to follow,

$$
W\left(\theta_{\mu}\right)=1-\cos ^{2}\left(\theta_{\mu}\right),
$$

where $\theta_{\mu}$ is the angle between the $\mu^{+}$and the direction of the $K^{ \pm}$in the $J / \psi$ rest frame [164]. The three dimensional angular distribution for the full $\chi_{c 1} \rightarrow$ $J / \psi \gamma \rightarrow \mu^{+} \mu^{-} \gamma$ cascade is significantly more complicated and is taken from [160]. 
The simulation provides a value of $\mathcal{A}_{B}=2.30 \pm 0.08$, where the uncertainty is due to the fitted parametrisation of the measured $B^{ \pm}$cross section and is estimated by varying the analytical function used to perform the fit.

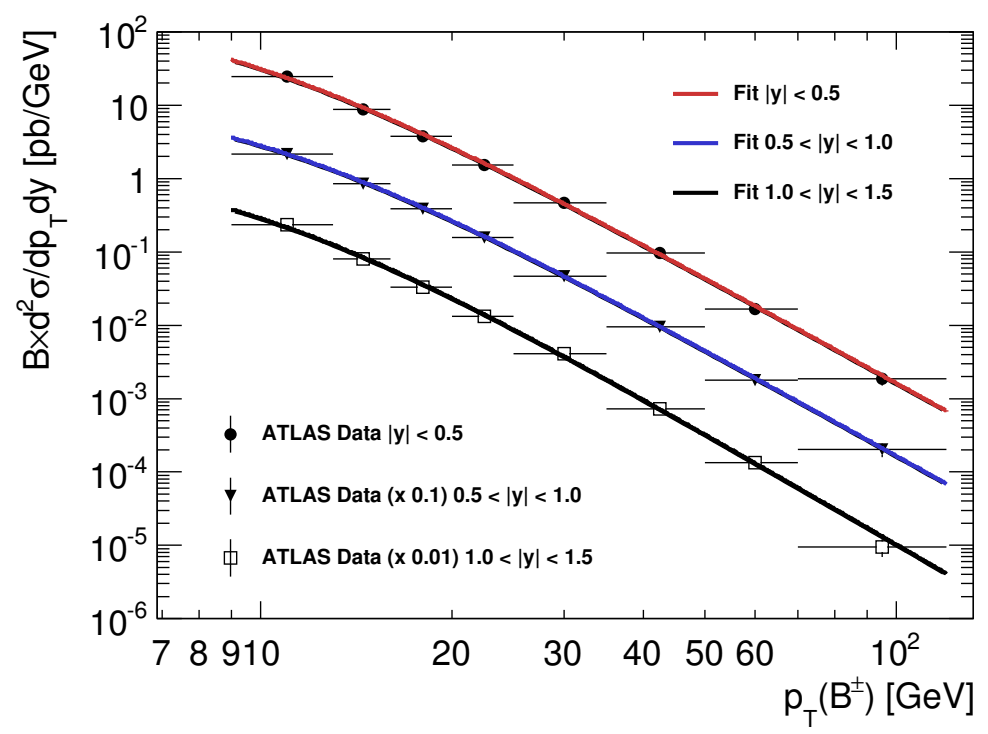

Figure 7.42: Fitted $B^{ \pm}$production differential cross sections, in three $B^{ \pm}$rapidity intervals. Data points from Ref. [163] and $B=\mathcal{B}\left(B^{ \pm} \rightarrow J / \psi K^{ \pm}\right) \cdot \mathcal{B}\left(J / \psi \rightarrow \mu^{+} \mu^{-}\right)$.

\subsubsection{Measurement of $N_{\chi_{c 1}}^{B}$ and $N_{J / \psi}^{B}$}

The measurement of $\mathcal{B}\left(B^{ \pm} \rightarrow \chi_{c 1} K^{ \pm}\right)$relies only on an accurate measurement of the ratio $N_{\chi_{c 1}}^{B} / N_{J / \psi}^{B}$, rather than the absolute yields themselves. The measurement of this ratio offers the advantage that some of the efficiencies and systematic uncertainties that contribute to the two individual yields cancel to a good approximation in the ratio. However, the kinematic differences between the two channels can give rise to efficiency ratios that are inconsistent with unity. In particular, the muon identification and trigger efficiencies do not reach their plateau values until around $p_{T}^{\mu}>8 \mathrm{GeV}$. While these two efficiencies do cancel to a good first approximation in the ratio, the differing kinematics of the $J / \psi \rightarrow \mu^{+} \mu^{-}$decays in each channel lead to a small residual imbalance that can be corrected for. The kinematics of the charged kaons in the two decay channels also differ slightly; however, the reconstruction effi- 
ciency for charged tracks reaches a plateau far below $p_{T}^{K}=3 \mathrm{GeV}$ (see Figure 5.3). The ratio of reconstruction efficiencies for the $K^{ \pm}$tracks within the fiducial region is verified to be consistent with unity to within $1 \%$ from simulation studies. The $\mu^{+} \mu^{-} K^{ \pm}$vertex quality cut has a weak dependence on the transverse momentum of the $\mu^{+} \mu^{-} K^{ \pm}$system, but is also verified to be consistent with unity to within $\pm 1 \%$, based on the studies performed in [163].

Each reconstructed $B^{ \pm} \rightarrow \chi_{c 1} K^{ \pm}$and $B^{ \pm} \rightarrow J / \psi K^{ \pm}$candidate is assigned a weight to correct for the residual effects of the muon identification and trigger efficiencies on the ratio $N_{\chi_{c 1}}^{B} / N_{J / \psi}^{B}$. The weight for $B^{ \pm} \rightarrow \chi_{c 1} K^{ \pm}$decays also includes a correction for converted photon reconstruction efficiency and the mass difference cuts described in Section 7.10.7. The reconstruction and trigger efficiency corrections used in this measurement are identical to those used in the main $\chi_{c}$ analysis and are described in detail in Section 7.5.

\subsubsection{Fitting Procedure}

The corrected $B^{ \pm} \rightarrow \chi_{c 1} K^{ \pm}$and $B^{ \pm} \rightarrow J / \psi K^{ \pm}$yields, $N_{\chi_{c 1}}^{B}$ and $N_{J / \psi}^{B}$, respectively, are measured with two independent weighted unbinned maximum likelihood fits to the mass difference distributions $\tilde{m}_{\chi_{c 1}}=m\left(\mu^{+} \mu^{-} \gamma K^{ \pm}\right)-m\left(\mu^{+} \mu^{-} \gamma\right)+m_{\chi_{c 1}}$ and $\tilde{m}_{J / \psi}=m\left(\mu^{+} \mu^{-} K^{ \pm}\right)-m\left(\mu^{+} \mu^{-}\right)+m_{J / \psi}$ (where $m_{\chi_{c 1}}$ and $m_{J / \psi}$ are the world average values of the $\chi_{c 1}$ and $J / \psi$ masses [3]) of reconstructed $B^{ \pm} \rightarrow \chi_{c 1} K^{ \pm}$and $B^{ \pm} \rightarrow J / \psi K^{ \pm}$candidates, respectively.

The mass difference distribution, $\tilde{m}_{\chi_{c 1}}$, is modelled by a PDF containing a component for the $B^{ \pm} \rightarrow \chi_{c 1} K^{ \pm}$signal and a background component. The signal component is described by a Gaussian PDF with a mean value and width that are both freely determined by the fit. The form of the background component is derived from a sample of simulated $p p \rightarrow b \bar{b} X \rightarrow J / \psi X^{\prime}$ events (with $B^{ \pm} \rightarrow \chi_{c 1} K^{ \pm}$ decays removed) processed with the selection cuts described in Section 7.10.7. The Gaussian kernel estimation procedure [123] is performed on the $\tilde{m} \chi_{c 1}$ distribution 
of these events to form a semi-analytical PDF background template used to model the background contribution to the $\tilde{m}_{\chi_{c 1}}$ distribution in data.

The mass difference distribution $\tilde{m}_{J / \psi}$ is modelled by a PDF containing a component for the $B^{ \pm} \rightarrow J / \psi K^{ \pm}$signal and a background component. The signal component is described by a double Gaussian PDF with a free mean value (common to both Gaussian PDFs). The relative normalisation of the two Gaussians and their independent width parameters are all free parameters. The background contribution to the $\tilde{m}_{J / \psi}$ distribution is modelled by three components. Partially reconstructed $B \rightarrow J / \psi K^{\star}\left(K^{ \pm} \pi^{\mp}\right)$ and $B \rightarrow \chi_{c 1,2} K$ decays form a structure at values of $\tilde{m}_{J / \psi}<5.2 \mathrm{GeV}$ that is modelled by the sum of a Gaussian (modelling the tail of the $B \rightarrow \chi_{c 1,2} K$ contribution) and a complementary error function (modelling the bulk of the $B \rightarrow J / \psi K^{\star}\left(K^{ \pm} \pi^{\mp}\right)$ contribution), as described in [163]. $B^{ \pm} \rightarrow J / \psi \pi^{ \pm}$ decays wrongly identified as $B^{ \pm} \rightarrow J / \psi K^{ \pm}$decays (i.e. the charged pion is assigned the charged kaon mass) lead to a peaking structure around $\tilde{m}_{J / \psi} \approx 5.38 \mathrm{GeV}$ that is modelled with a CB function, as described in [163]. The non-resonant combinatoric background is modelled with an exponential function.

The fit results to the $\tilde{m}_{\chi_{c 1}}$ and $\tilde{m}_{J / \psi}$ distributions are shown in Figure 7.43. 

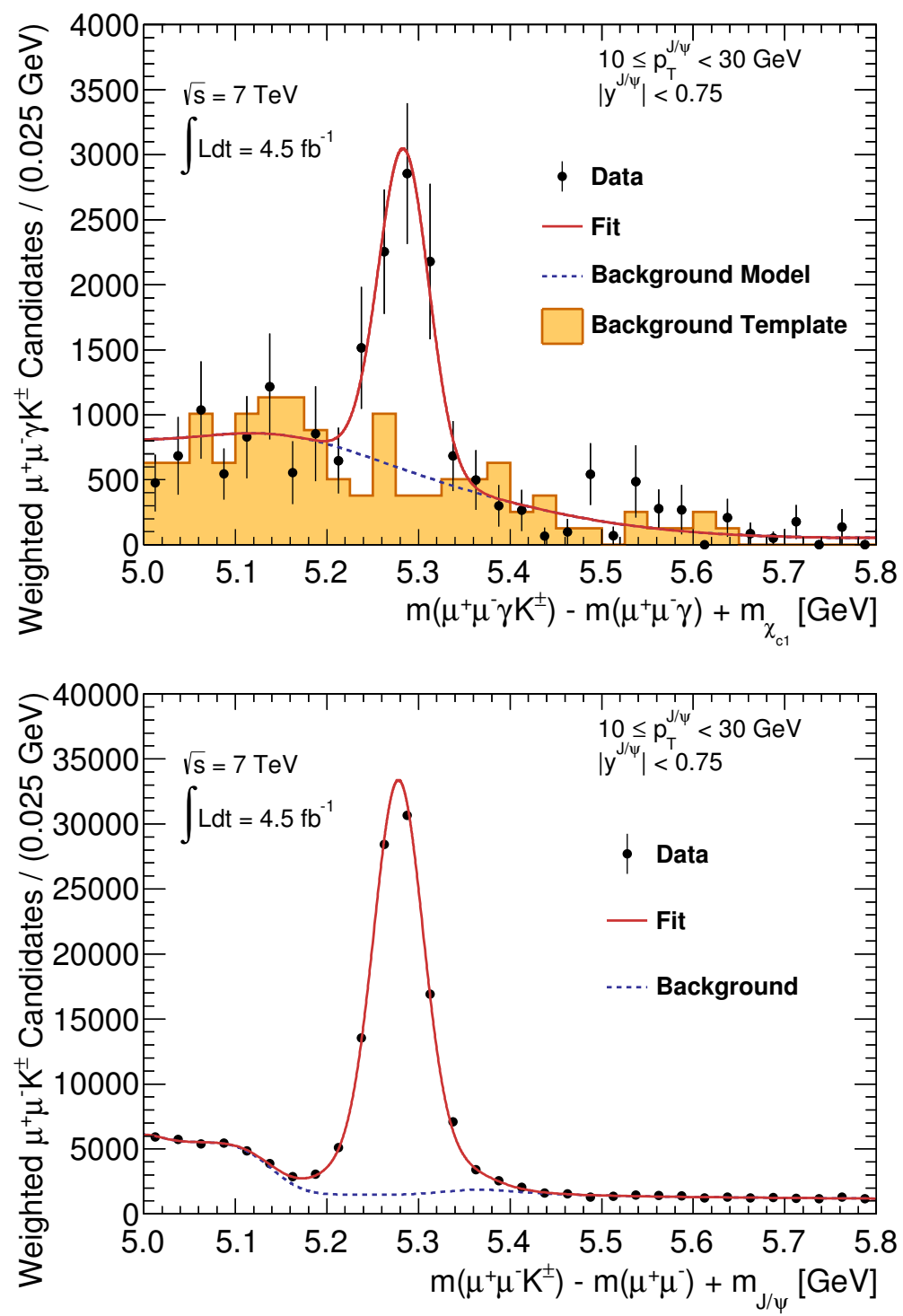

Figure 7.43: The results of weighted unbinned maximum likelihood fits to the $\tilde{m} \chi_{c 1}$ (top) and $\tilde{m}_{J / \psi}$ (bottom) distributions of reconstructed $B^{ \pm} \rightarrow \chi_{c 1} K^{ \pm}$and $B^{ \pm} \rightarrow$ $J / \psi K^{ \pm}$decays, respectively. 


\subsubsection{Systematic Uncertainties}

Several sources of systematic uncertainty on the measurement of the ratio $N_{\chi_{c 1}}^{B} / N_{J / \psi}^{B}$ are considered. The systematic uncertainties on the muon identification, trigger and conversion reconstruction efficiency corrections are estimated as described in Section 7.8 for the main $\chi_{c}$ analysis. The systematic uncertainty on the fitting procedure used to measure $N_{\chi_{c 1}}^{B} / N_{J / \psi}^{B}$ is estimated by repeating the fit with several modifications. These modifications include using alternative signal PDFs (double Gaussian for $B^{ \pm} \rightarrow \chi_{c 1} K^{ \pm}$and Gaussian convolved with a Breit-Winger distribution for $\left.B^{ \pm} \rightarrow J / \psi K^{ \pm}\right)$and alternative background parametrisations. In the case of the $B^{ \pm} \rightarrow \chi_{c 1} K^{ \pm}$fit, the parameter $\rho$, controlling the structure retention of the Gaussian kernel density estimation background PDF is varied [123]. The $B^{ \pm} \rightarrow J / \psi K^{ \pm}$ background model is modified by varying the shape of the $B \rightarrow J / \psi K^{\star}\left(K^{ \pm} \pi^{\mp}\right)$ contribution and removing the $B^{ \pm} \rightarrow J / \psi \pi^{ \pm}$component. The fits to both distributions are also performed with different fit ranges in $\tilde{m}_{\chi_{c 1}}$ and $\tilde{m}_{J / \psi}$. The average of the absolute deviations in the alternative fit results from the nominal result for the variations studied is used to estimate the systematic uncertainty on the fit procedure. The systematic uncertainty on the acceptance correction $\mathcal{A}_{B}$ is described in Section 7.10.8. The systematic uncertainties on the external branching fractions in Equation 7.20 are neglected as they are small in comparison to the total experimental uncertainty. Table 7.5 shows the individual contributions to the total systematic uncertainty on the measurement of $\mathcal{B}\left(B^{ \pm} \rightarrow \chi_{c 1} K^{ \pm}\right)$.

\subsubsection{Result and Conclusion}

The measured value of the branching fraction for the decay $B^{ \pm} \rightarrow \chi_{c 1} K^{ \pm}$is $\mathcal{B}\left(B^{ \pm} \rightarrow \chi_{c 1} K^{ \pm}\right)=(4.9 \pm 0.9$ (stat.) \pm 0.6 (syst.) $) \times 10^{-4}$. This value is in good agreement with the world average value of $(4.79 \pm 0.26) \times 10^{-4}[3]$. This agreement suggests that the analysis procedure and conversion reconstruction efficiency, 


\begin{tabular}{|l|c|}
\hline & Fractional Uncertainty [\%] \\
\hline Converted-photon reconstruction efficiency & 10 \\
Conversion probability & 4 \\
Muon reconstruction efficiency & 1 \\
Trigger efficiency & 1 \\
Acceptance & 3 \\
Fit model & 6 \\
\hline Statistical & 18 \\
Systematic & 13 \\
\hline Total & 22 \\
\hline
\end{tabular}

Table 7.5: A breakdown of the sources of systematic uncertainty on the measurement of $\mathcal{B}\left(B^{ \pm} \rightarrow \chi_{c 1} K^{ \pm}\right)$.

derived from simulation, are accurate at the level of around $\pm 20 \%$ (the total uncertainty on the measurement, after subtracting in quadrature the contribution from the conversion reconstruction efficiency). The total uncertainty on the measurement is dominated by the statistical uncertainty. While the precision of this measurement is far poorer than the measurements of Belle [161] and BaBar [162], it represents the most precise measurement of this branching fraction to be performed at a hadron collider, with the only previous measurement being from CDF: $(15.5 \pm 5.4$ (stat.) \pm 2.0 (syst.) $) \times 10^{-4}[165]$. The result is compared to the worldaverage and the measurements of Belle, BaBar and CDF in Figure 7.44. 


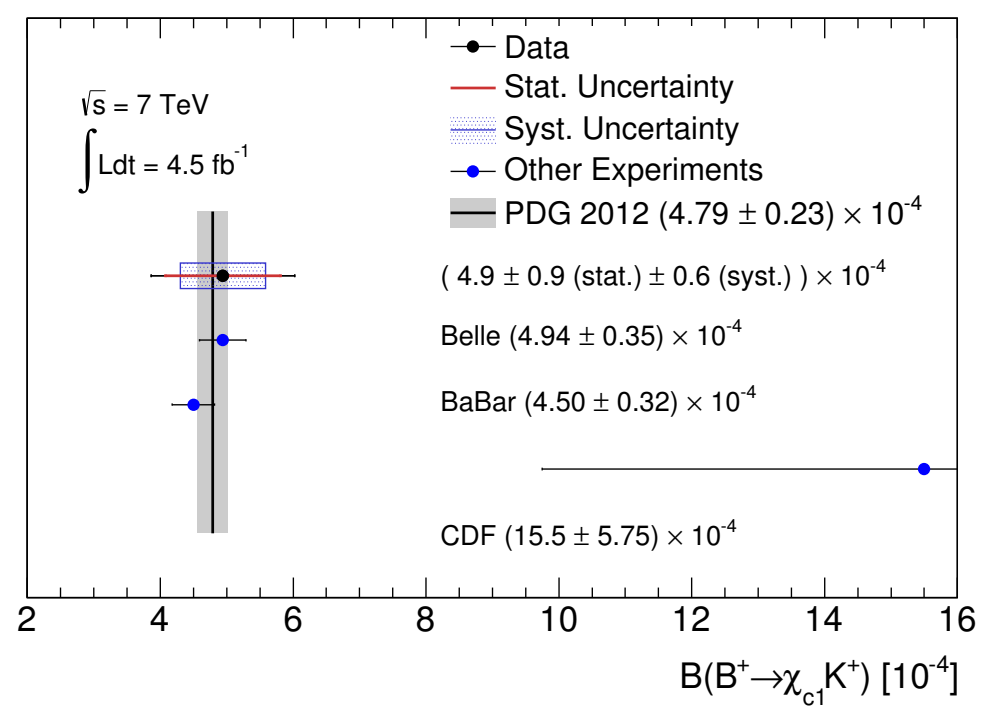

Figure 7.44: The measured value of $\mathcal{B}\left(B^{ \pm} \rightarrow \chi_{c 1} K^{ \pm}\right)$compared to the world average and the measurements of Belle, BaBar and CDF [3,161,162,165]. 


\subsection{Conclusion}

The $\chi_{c J}(1 P)$ charmonium states are reconstructed from a sample of $\sqrt{s}=7 \mathrm{TeV}$ $p p$ collision data recorded by the ATLAS experiment, representing an integrated luminosity of $4.5 \mathrm{fb}^{-1}$. The differential cross sections for the prompt and non-prompt production of the $\chi_{c 1}$ and $\chi_{c 2}$ states are measured as functions of both $p_{T}^{\chi_{c}}$ and $p_{T}^{J / \psi}$ within the region $\left|y^{J / \psi}\right|<0.75$. These measurements are combined with the ATLAS measurement of prompt $J / \psi$ production in $\sqrt{s}=7 \mathrm{TeV} p p$ collisions to estimate the fraction of prompt $J / \psi$ produced in feed-down from radiative $\chi_{c J}$ decays, measured as a function of $p_{T}^{J / \psi}$. Between $24-28 \%$ of prompt $J / \psi$ are measured to be produced in radiative $\chi_{c J}$ decays for $10<p_{T}^{J / \psi}<30 \mathrm{GeV}$. The production cross section of the $\chi_{c 2}$ state, relative to the $\chi_{c 1}$ state, is measured for both prompt and non-prompt production as a function of $p_{T}^{J / \psi}$. These measurements of prompt $\chi_{c 1}$ and $\chi_{c 2}$ production are compared to various theoretical models of prompt charmonium production and to existing measurements. The predictions of NLO NRQCD consistently provide the best description of the prompt $\chi_{c}$ production measurements. The fractions of $\chi_{c 1}$ and $\chi_{c 2}$ produced in the decays of $b$-hadrons are also measured. The non-prompt $\chi_{c 1}$ and $\chi_{c 2}$ production cross sections are in general agreement with the theoretical predictions of a model based upon the FONLL approach to b-hadron production, though the measurements tend to lie below the predictions at high $p_{T}$. This could suggest that the contributions from $B_{s}^{0}$ and $b$-baryon decays to final states including the $\chi_{c 1}$ and $\chi_{c 2}$ states, not described by the model, may be important. 


\section{Chapter 8 \\ CONCLUSION}

Our theoretical understanding of the production of quarkonium states in hadronic interactions is still not entirely satisfactory, and no single theoretical approach can claim to fully describe the abundance of measurements that now exists. The arrival of the Large Hadron Collider, capable of delivering unprecedentedly high energy collisions and high luminosities to its cutting edge experiments, provides a unique opportunity to significantly advance our understanding of quarkonium production. The aim of this thesis is to contribute to this effort by making two measurements of the $\chi_{b}$ and $\chi_{c}$ quarkonium states in $p p$ collisions at the ATLAS experiment.

The production of the $\chi_{b}(1 P)$ and $\chi_{b}(2 P)$ bottomonium states is observed in a sample of $\sqrt{s}=7 \mathrm{TeV} p p$ collision data, representing an integrated luminosity of $4.4 \mathrm{fb}^{-1}$. The $\chi_{b}$ states are reconstructed through the radiative decay $\chi_{b} \rightarrow \Upsilon(1 S) \gamma$ (with $\Upsilon(1 S) \rightarrow \mu^{+} \mu^{-}$), where photons are reconstructed from energy deposits in the ATLAS electromagnetic calorimeter. Within the same data sample, a candidate for a new bottomonium state is observed, consistent with theoretical expectations for the $\chi_{b}(3 P)$ states. The production-averaged mass barycentre for the $\chi_{b}(3 P)$ candidate is measured to be $10541 \pm 11$ (stat.) \pm 30 (syst.) $\mathrm{MeV}$. An independent observation of this new state, based upon an alternative analysis of the same data sample, is also discussed. The observation of a new bottomonium state decaying to $\Upsilon(1 S) \gamma$, consistent with that described here, has subsequently been confirmed by both the D0 and LHCb experiments. The consequences of this discovery for our 
understanding of bottomonium production phenomenology in hadron collisions is reviewed.

The production of the $\chi_{c 1}$ and $\chi_{c 2}$ charmonium states has been measured in $\sqrt{s}=7 \mathrm{TeV} p p$ collisions with the ATLAS experiment using a data sample representing an integrated luminosity of $4.5 \mathrm{fb}^{-1}$. The prompt and non-prompt production cross sections for the $\chi_{c 1}$ and $\chi_{c 2}$ states are measured within the region $\left|y^{J / \psi}\right|<0.75$. These measurements suggest that $24-28 \%$ of prompt $J / \psi$ are produced in feed-down from radiative $\chi_{c 1}$ and $\chi_{c 2}$ decays. The production of the $\chi_{c 2}$ state, relative to the $\chi_{c 1}$ state, is measured for both prompt and non-prompt production processes. These measurements are also used to determine the fraction of $\chi_{c 1}$ and $\chi_{c 2}$ produced in the decays of $b$-hadrons. This collection of measurements is compared to a number of theoretical predictions for $\chi_{c J}$ production at the LHC. The predictions of NLO NRQCD are found to consistently provide a good description of the measurements, while alternative approaches based on the CSM are found to be in significant disagreement with the data. The non-prompt $\chi_{c 1}$ and $\chi_{c 2}$ production cross sections are in general agreement with the theoretical predictions of a model based upon the FONLL approach to $b$-hadron production, though the measurements tend to lie below the predictions at high $p_{T}$. This could suggest that the contributions from $B_{s}^{0}$ and $b$-baryon decays to final states including the $\chi_{c 1}$ and $\chi_{c 2}$ states, not described by the model, may be important.

The measurements presented here are performed with a small fraction (less than $20 \%$ ) of the total ATLAS Run I dataset. The full Run I dataset offers many further opportunities to study various aspects of $\chi_{c}$ and $\chi_{b}$ production, in addition to many other aspects of quarkonium production. Further studies of the $\chi_{b}(3 P)$ candidate are necessary to determine its nature. In particular, a full angular analysis of $\chi_{b} \rightarrow$ $\Upsilon(1 S) \gamma$ could potentially be used to determine the unknown quantum number of the state(s) to confirm or exclude the $\chi_{b}(3 P)$ hypothesis. A larger data sample may also allow any fine structure to be resolved; this could determine whether the 
structure observed represents a triplet of states (which would support the $\chi_{b J}(3 P)$ hypothesis) or a single state. The larger data sample could allow the polarisation of the $\chi_{c J}$ states to be measured. This would significantly reduce the systematic uncertainty on the measurements presented here and would represent a significant step forwards in the understanding of quarkonium polarisation at the LHC. 


\section{References}

[1] E598 Collaboration, J. J. Aubert et al., Experimental Observation of a Heavy Particle J, Phys. Rev. Lett. 33 (1974) 1404-1406.

[2] SLAC-SP-017 Collaboration, J. E. Augustin et al., Discovery of a Narrow Resonance in $e^{+} e^{-}$Annihilation, Phys. Rev. Lett. 33 (1974) 1406-1408.

[3] Particle Data Group, J. Beringer et al., Review of Particle Physics (RPP), Phys. Rev. D86 (2012) 010001.

[4] ATLAS Collaboration, G. Aad et al., Observation of a new particle in the search for the Standard Model Higgs boson with the ATLAS detector at the LHC, Phys. Lett. B716 (2012) 1-29, [arXiv:1207.7214].

[5] CMS Collaboration, S. Chatrchyan et al., Observation of a new boson at a mass of $125 \mathrm{GeV}$ with the CMS experiment at the LHC, Phys. Lett. B716 (2012) 30-61, [arXiv:1207.7235].

[6] LHC Higgs Cross Section Working Group, S. Heinemeyer et al., Handbook of LHC Higgs Cross Sections: 3. Higgs Properties, arXiv: 1307.1347.

[7] M. E. Peskin and D. V. Schroeder, An Introduction to quantum field theory. Westview Press, 1995.

[8] R. K. Ellis, W. J. Stirling, and B. R. Webber, QCD and Collider Physics. Cambridge University Press, 1996.

[9] S. Narison, QCD as a theory of hadrons: From partons to confinement. Cambridge University Press, 2002.

[10] S. W. Herb et al., Observation of a dimuon resonance at $9.5 \mathrm{GeV}$ in $400 \mathrm{GeV}$ proton - nucleus collisions, Phys. Rev. Lett. 39 (1977) 252-255.

[11] BaBar Collaboration, B. Aubert et al., Observation of the bottomonium ground state in the decay $\Upsilon(3 S) \rightarrow \gamma \eta_{b}$, Phys. Rev. Lett. 101 (2008) 071801, [arXiv:0807.1086].

[12] Belle Collaboration, R. Mizuk et al., Evidence for the $\eta_{b}(2 S)$ and observation of $h_{b}(1 P) \rightarrow \eta_{b}(1 S) \gamma$ and $h_{b}(2 P) \rightarrow \eta_{b}(1 S) \gamma$, Phys. Rev. Lett. 109 (2012) 232002, [arXiv:1205.6351]. 
[13] C. Davies, K. Hornbostel, G. Lepage, A. Lidsey, J. Shigemitsu, et al., Precision charmonium spectroscopy from lattice QCD, Phys. Rev. D52 (1995) 6519-6529, [hep-lat/9506026].

[14] HPQCD Collaboration, R. Dowdall et al., The Upsilon spectrum and the determination of the lattice spacing from lattice QCD including charm quarks in the sea, Phys. Rev. D85 (2012) 054509, [arXiv:1110.6887].

[15] HPQCD Collaboration, J. Daldrop, C. Davies, and R. Dowdall, Prediction of the bottomonium D-wave spectrum from full lattice QCD, Phys. Rev. Lett. 108 (2012) 102003, [arXiv:1112.2590].

[16] R. Eisberg and R. Resnick, Quantum Physics. Wiley, 2nd ed., 1985.

[17] Quarkonium Working Group, N. Brambilla et al., Heavy quarkonium physics, hep-ph/0412158.

[18] E. Eichten, K. Gottfried, T. Kinoshita, K. Lane, and T.-M. Yan, Charmonium: The Model, Phys. Rev. D17 (1978) 3090.

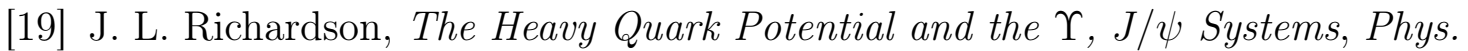
Lett. B82 (1979) 272.

[20] D. Ebert, R. Faustov, and V. Galkin, Properties of heavy quarkonia and $B_{c}$ mesons in the relativistic quark model, Phys. Rev. D67 (2003) 014027, [hep-ph/0210381].

[21] S. Godfrey and N. Isgur, Mesons in a Relativized Quark Model with Chromodynamics, Phys. Rev. D32 (1985) 189-231.

[22] Belle Collaboration, S. Choi et al., Observation of a narrow charmonium-like state in exclusive $B^{ \pm} \rightarrow K^{ \pm} \pi^{+} \pi^{-} J / \psi$ decays, Phys. Rev. Lett. 91 (2003) 262001, [hep-ex/0309032].

[23] CDF Collaboration, D. Acosta et al., Observation of the narrow state $X(3872) \rightarrow J / \psi \pi^{+} \pi^{-}$in $\bar{p} p$ collisions at $\sqrt{s}=1.96 \mathrm{TeV}$, Phys. Rev. Lett. 93 (2004) 072001, [hep-ex/0312021].

[24] Do Collaboration, V. Abazov et al., Observation and properties of the $X(3872)$ decaying to $\mathrm{J} / \psi \pi^{+} \pi^{-}$in $p \bar{p}$ collisions at $\sqrt{s}=1.96 \mathrm{TeV}$, Phys. Rev. Lett. 93 (2004) 162002, [hep-ex/0405004].

[25] N. Brambilla, S. Eidelman, B. Heltsley, R. Vogt, G. Bodwin, et al., Heavy quarkonium: progress, puzzles, and opportunities, Eur. Phys. J. C71 (2011) 1534, [arXiv:1010.5827].

[26] LHCb Collaboration, R. Aaij et al., Determination of the X(3872) meson quantum numbers, Phys. Rev. Lett. 110 (2013) 222001, [arXiv:1302.6269].

[27] CMS Collaboration, S. Chatrchyan et al., Measurement of the X(3872) production cross section via decays to $J / \psi \pi^{+} \pi^{-}$in pp collisions at $\sqrt{s}=7 \mathrm{TeV}$, JHEP 1304 (2013) 154, [arXiv:1302.3968]. 
[28] V. Gribov and L. Lipatov, Deep inelastic ep scattering in perturbation theory, Sov. J. Nucl. Phys. 15 (1972) 438-450.

[29] G. Altarelli and G. Parisi, Asymptotic Freedom in Parton Language, Nucl. Phys. B126 (1977) 298.

[30] Y. L. Dokshitzer, Calculation of the Structure Functions for Deep Inelastic Scattering and $e^{+} e^{-}$Annihilation by Perturbation Theory in Quantum Chromodynamics., Sov. Phys. JETP 46 (1977) 641-653.

[31] A. Martin, W. Stirling, R. Thorne, and G. Watt, Parton distributions for the LHC, Eur. Phys. J. C63 (2009) 189-285, [arXiv:0901.0002].

[32] MSTW Parton Distribution Function figures, http://mstwpdf .hepforge.org/. Accessed: 22/01/2014.

[33] H. Fritzsch, Producing Heavy Quark Flavors in Hadronic Collisions: A Test of Quantum Chromodynamics, Phys. Lett. B67 (1977) 217.

[34] F. Halzen, CVC for Gluons and Hadroproduction of Quark Flavors, Phys. Lett. B69 (1977) 105.

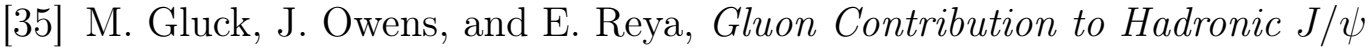
Production, Phys. Rev. D17 (1978) 2324.

[36] S. Digal, P. Petreczky, and H. Satz, Quarkonium feed down and sequential suppression, Phys. Rev. D64 (2001) 094015, [hep-ph/0106017].

[37] J. Amundson, O. J. Eboli, E. Gregores, and F. Halzen, Quantitative tests of color evaporation: Charmonium production, Phys. Lett. B390 (1997) 323-328, [hep-ph/9605295].

[38] CDF Collaboration, A. Abulencia et al., Measurement of $\sigma_{\chi_{c 2}} \mathcal{B}\left(\chi_{c 2} \rightarrow J / \psi \gamma\right) / \sigma_{\chi_{c 1}} \mathcal{B}\left(\chi_{c 1} \rightarrow J / \psi \gamma\right)$ in $p \bar{p}$ collisions at $\sqrt{s}=1.96 \mathrm{TeV}$, Phys. Rev. Lett. 98 (2007) 232001, [hep-ex/0703028].

[39] LHCb Collaboration, R. Aaij et al., Measurement of the cross-section ratio $\sigma\left(\chi_{c 2}\right) / \sigma\left(\chi_{c 1}\right)$ for prompt $\chi_{c}$ production at $\sqrt{s}=7 \mathrm{TeV}$, Phys. Lett. B714 (2012) 215-223, [arXiv:1202.1080].

[40] CMS Collaboration, S. Chatrchyan et al., Measurement of the relative prompt production rate of $\chi_{c 2}$ and $\chi_{c 1}$ in pp collisions at $\sqrt{s}=7 \mathrm{TeV}$, Eur. Phys. J. C72 (2012) 2251, [arXiv:1210.0875].

[41] G. T. Bodwin, E. Braaten, and J. Lee, Comparison of the color-evaporation model and the NRQCD factorization approach in charmonium production, Phys. Rev. D72 (2005) 014004, [hep-ph/0504014].

[42] R. Baier and R. Ruckl, Hadronic Production of $J / \psi$ and $\Upsilon$ : Transverse Momentum Distributions, Phys. Lett. B102 (1981) 364. 
[43] R. Baier and R. Ruckl, Hadronic Collisions: A Quarkonium Factory, Z. Phys. C19 (1983) 251.

[44] E. L. Berger and D. L. Jones, Inelastic Photoproduction of $J / \psi$ and $\Upsilon$ by Gluons, Phys. Rev. D23 (1981) 1521-1530.

[45] C.-H. Chang, Hadronic production of $J / \psi$ associated with a gluon, Nucl. Phys. B172 (1980) 425-434.

[46] G. A. Schuler, Quarkonium production and decays, Phys. Rept. (1994) [hep-ph/9403387].

[47] CDF Collaboration, F. Abe et al., $J / \psi$ and $\psi(2 S)$ production in $p \bar{p}$ collisions at $\sqrt{s}=1.8 \mathrm{TeV}$, Phys. Rev. Lett. 79 (1997) 572-577.

[48] J. Lansberg, $J / \psi$ production at $\sqrt{s}=1.96$ and $7 \mathrm{TeV}$ : Color-Singlet Model, NNLO* and polarisation, J. Phys. G38 (2011) 124110, [arXiv:1107.0292].

[49] P. Hagler, R. Kirschner, A. Schafer, L. Szymanowski, and O. Teryaev, Towards a solution of the charmonium production controversy: $k^{-}$ perpendicular factorization versus color octet mechanism, Phys. Rev. Lett. 86 (2001) 1446-1449, [hep-ph/0004263].

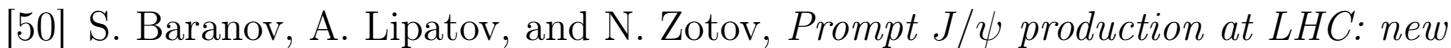
evidence for the $k_{t}$-factorization, Phys. Rev. D85 (2012) 014034, [arXiv:1108.2856].

[51] S. Baranov, On the $\sigma\left(\chi_{c 2}\right) / \sigma\left(\chi_{c 1}\right)$ ratio in the $k_{T}$-factorization approach, Phys. Rev. D83 (2011) 034035.

[52] G. T. Bodwin, E. Braaten, and G. P. Lepage, Rigorous QCD analysis of inclusive annihilation and production of heavy quarkonium, Phys. Rev. D 51 (Feb, 1995) 1125-1171.

[53] W. Caswell and G. Lepage, Effective Lagrangians for Bound State Problems in QED, QCD, and Other Field Theories, Phys. Lett. B167 (1986) 437.

[54] CDF Collaboration, F. Abe et al., Production of $J / \psi$ mesons from $\chi_{c}$ meson decays in p $\bar{p}$ collisions at $\sqrt{s}=1.8 \mathrm{TeV}$, Phys. Rev. Lett. 79 (1997) $578-583$.

[55] M. Kramer, Quarkonium production at high-energy colliders, Prog. Part. Nucl. Phys. 47 (2001) 141-201, [hep-ph/0106120].

[56] M. Cacciari, S. Frixione, N. Houdeau, M. L. Mangano, P. Nason, et al., Theoretical predictions for charm and bottom production at the LHC, JHEP 1210 (2012) 137, [arXiv: 1205.6344].

[57] V. Kartvelishvili, A. Likhoded, and V. Petrov, On the Fragmentation Functions of Heavy Quarks Into Hadrons, Phys. Lett. B78 (1978) 615. 
[58] C. Peterson, D. Schlatter, I. Schmitt, and P. M. Zerwas, Scaling Violations in Inclusive $e^{+} e^{-}$Annihilation Spectra, Phys. Rev. D27 (1983) 105.

[59] OPAL Collaboration, G. Abbiendi et al., Inclusive analysis of the $b$ quark fragmentation function in $Z$ decays at LEP, Eur. Phys. J. C29 (2003) 463-478, [hep-ex/0210031].

[60] BaBar Collaboration, Study of inclusive production of charmonium mesons in b decays, Phys. Rev. D 67 (Feb, 2003) 032002.

[61] B. A. Kniehl and G. Kramer, Inclusive $J / \psi$ and $\psi(2 S)$ production from $B$

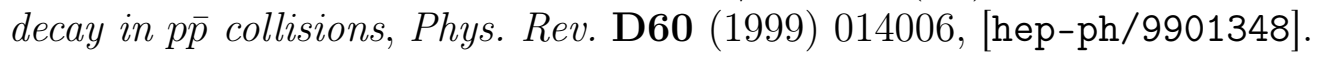

[62] P. Faccioli, C. Lourenco, J. Seixas, and H. K. Wohri, Determination of $\chi_{c}$ and $\chi_{b}$ polarizations from dilepton angular distributions in radiative decays, Phys. Rev. D83 (2011) 096001, [arXiv:1103.4882].

[63] P. Faccioli, C. Lourenco, J. Seixas, and H. K. Wohri, Towards the experimental clarification of quarkonium polarization, Eur. Phys. J. C69 (2010) 657-673, [arXiv: 1006.2738].

[64] P. Faccioli, C. Lourenco, J. Seixas, and H. Woehri, Study of $\psi^{\prime}$ and $\chi_{c}$ decays as feed-down sources of $J / \psi$ hadro-production, JHEP 0810 (2008) 004, [arXiv:0809.2153].

[65] CDF Collaboration, T. Affolder et al., Production of $\Upsilon(1 S)$ mesons from $\chi_{b}$ decays in $p \bar{p}$ collisions at $\sqrt{s}=1.8 \mathrm{TeV}$, Phys. Rev. Lett. 84 (2000) 2094-2099, [hep-ex/9910025].

[66] ATLAS Collaboration, G. Aad et al., Observation of a new $\chi_{b}$ state in radiative transitions to $\Upsilon(1 S)$ and $\Upsilon(2 S)$ at ATLAS, Phys. Rev. Lett. 108 (2012) 152001, [arXiv:1112.5154].

[67] S. Baranov, Prompt $\Upsilon(n S)$ production at the LHC in view of the $k_{t}$-factorization approach, Phys. Rev. D86 (2012) 054015.

[68] CDF Collaboration, D. Acosta et al., Measurement of the $J / \psi$ meson and b-hadron production cross sections in pp collisions at $\sqrt{s}=1960 \mathrm{GeV}$, Phys. Rev. D71 (2005) 032001, [hep-ex/0412071].

[69] CDF Collaboration, T. Aaltonen et al., Production of $\psi(2 S)$ Mesons in $p \bar{p}$ Collisions at $\sqrt{s}=1.96 \mathrm{TeV}$, Phys. Rev. D80 (2009) 031103, [arXiv:0905.1982].

[70] Do Collaboration, S. Abachi et al., $J / \psi$ production in $p \bar{p}$ collisions at $\sqrt{s}=1.8 \mathrm{TeV}$, Phys. Lett. B370 (1996) 239-248.

[71] CDF Collaboration, A. Abulencia et al., Polarization of $J / \psi$ and $\psi(2 S)$ mesons produced in $p \bar{p}$ collisions at $\sqrt{s}=1.96 \mathrm{TeV}$, Phys. Rev. Lett. 99 (2007) 132001, [arXiv:0704.0638]. 
[72] Do Collaboration, V. Abazov et al., Measurement of inclusive differential cross sections for $\Upsilon(1 S)$ production in pp collisions at $\sqrt{s}=1.96 \mathrm{TeV}$, Phys. Rev. Lett. 94 (2005) 232001, [hep-ex/0502030].

[73] CDF Collaboration, D. Acosta et al., $\Upsilon$ production and polarization in $p \bar{p}$ collisions at $\sqrt{s}=1.8 \mathrm{TeV}$, Phys. Rev. Lett. 88 (2002) 161802.

[74] D0 Collaboration, V. Abazov et al., Measurement of the polarization of the $\Upsilon(1 S)$ and $\Upsilon(2 S)$ states in $p \bar{p}$ collisions at $\sqrt{s}=1.96 \mathrm{TeV}$, Phys. Rev. Lett. 101 (2008) 182004, [arXiv:0804.2799].

[75] CDF Collaboration, T. Aaltonen et al., Measurements of Angular Distributions of Muons From $\Upsilon$ Meson Decays in $p \bar{p}$ Collisions at $\sqrt{s}=1.96 \mathrm{TeV}$, Phys. Rev. Lett. 108 (2012) 151802, [arXiv:1112.1591].

[76] ALICE Collaboration, K. Aamodt et al., Rapidity and transverse momentum dependence of inclusive $J / \psi$ production in pp collisions at $\sqrt{s}=7 \mathrm{TeV}$, Phys. Lett. B704 (2011) 442-455, [arXiv:1105.0380].

[77] ATLAS Collaboration, G. Aad et al., Measurement of the differential cross-sections of inclusive, prompt and non-prompt $J / \psi$ production in proton-proton collisions at $\sqrt{s}=7 \mathrm{TeV}$, Nucl. Phys. B850 (2011) 387-444, [arXiv: 1104.3038].

[78] CMS Collaboration, S. Chatrchyan et al., $J / \psi$ and $\psi(2 S)$ production in pp collisions at $\sqrt{s}=7 \mathrm{TeV}$, JHEP 1202 (2012) 011, [arXiv:1111.1557].

[79] LHCb Collaboration, R. Aaij et al., Measurement of $J / \psi$ production in $p p$ collisions at $\sqrt{s}=7 \mathrm{TeV}$, Eur. Phys. J. C71 (2011) 1645,

[arXiv: 1103.0423].

[80] LHCb Collaboration, R. Aaij et al., Production of $J / \psi$ and $\Upsilon$ mesons in pp collisions at $\sqrt{s}=8 \mathrm{TeV}$, JHEP 1306 (2013) 064, [arXiv:1304.6977].

[81] LHCb Collaboration, R. Aaij et al., Measurement of $\psi(2 S)$ meson production in pp collisions at $\sqrt{s}=7 \mathrm{TeV}$, Eur. Phys. J. C72 (2012) 2100, [arXiv: 1204.1258].

[82] LHCb Collaboration, R. Aaij et al., Measurement of the ratio of prompt $\chi_{c}$ to $\mathrm{J} / \psi$ production in pp collisions at $\sqrt{s}=7 \mathrm{TeV}$, Phys. Lett. B718 (2012) 431-440, [arXiv: 1204.1462].

[83] CMS Collaboration, S. Chatrchyan et al., Measurement of the prompt $J / \psi$ and $\psi(2 S)$ polarizations in pp collisions at $\sqrt{s}=7 \mathrm{TeV}$, Phys. Lett. B727 (2013) 381-402, [arXiv: 1307.6070].

[84] LHCb Collaboration, R. Aaij et al., Measurement of $J / \psi$ polarization in pp collisions at $\sqrt{s}=7 \mathrm{TeV}$, Eur. Phys. J. C73 (2013) 2631, [arXiv: 1307.6379]. 
[85] ALICE Collaboration, B. Abelev et al., $J / \psi$ polarization in pp collisions at $\sqrt{s}=7 \mathrm{TeV}$, Phys. Rev. Lett. 108 (2012) 082001, [arXiv:1111.1630].

[86] LHCb collaboration, R. Aaij et al., Updated measurements of exclusive $J / \psi$ and $\psi(2 S)$ production cross-sections in pp collisions at $\sqrt{s}=7 \mathrm{TeV}$, arXiv: 1401.3288.

[87] ATLAS Collaboration, G. Aad et al., Measurement of $\Upsilon$ production in $\sqrt{s}=7 \mathrm{TeV}$ pp collisions at ATLAS, Phys. Rev. D87 (2013), no. 5 052004, [arXiv:1211.7255].

[88] CMS Collaboration, S. Chatrchyan et al., Measurement of the $\Upsilon(1 S)$, $\Upsilon(2 S)$, and $\Upsilon(3 S)$ cross sections in pp collisions at $\sqrt{s}=7 \mathrm{TeV}$, Phys. Lett. B727 (2013) 101-125, [arXiv:1303.5900].

[89] LHCb Collaboration, R. Aaij et al., Measurement of $\Upsilon$ production in $p p$ collisions at $\sqrt{s}=7 \mathrm{TeV}$, Eur. Phys. J. C72 (2012) 2025, [arXiv: 1202.6579].

[90] LHCb Collaboration, R. Aaij et al., Measurement of the fraction of $\Upsilon(1 S)$ originating from $\chi_{b}(1 P)$ decays in pp collisions at $\sqrt{s}=7 \mathrm{TeV}$, JHEP 1211 (2012) 031, [arXiv: 1209.0282].

[91] CMS Collaboration, S. Chatrchyan et al., Measurement of the $\Upsilon(1 S)$, $\Upsilon(2 S)$ and $\Upsilon(3 S)$ polarizations in pp collisions at $\sqrt{s}=7 \mathrm{TeV}$, Phys. Rev. Lett. 110 (2013) 081802, [arXiv:1209.2922].

[92] L. Evans and P. Bryant, LHC Machine, JINST 3 (2008) S08001.

[93] ATLAS Collaboration, G. Aad et al., The ATLAS Experiment at the CERN Large Hadron Collider, JINST 3 (2008) S08003.

[94] ATLAS Collaboration, G. Aad et al., The ATLAS Inner Detector commissioning and calibration, Eur. Phys. J. C70 (2010) 787-821, [arXiv: 1004.5293].

[95] ATLAS Collaboration, G. Aad et al., Expected Performance of the ATLAS Experiment - Detector, Trigger and Physics, SLAC-R-980, CERN-OPEN-2008-020.

[96] ATLAS Collaboration, G. Aad et al., Readiness of the ATLAS Liquid Argon Calorimeter for LHC Collisions, Eur. Phys. J. C70 (2010) 723-753, [arXiv:0912.2642].

[97] ATLAS Collaboration, G. Aad et al., Readiness of the ATLAS Tile Calorimeter for LHC collisions, Eur. Phys. J. C70 (2010) 1193-1236, [arXiv: 1007.5423].

[98] ATLAS Collaboration, G. Aad et al., Commissioning of the ATLAS Muon Spectrometer with Cosmic Rays, Eur. Phys. J. C70 (2010) 875-916, [arXiv: 1006 . 4384]. 
[99] T. Sjostrand, S. Mrenna, and P. Z. Skands, PYTHIA 6.4 Physics and Manual, JHEP 0605 (2006) 026, [hep-ph/0603175].

[100] M. Bahr, S. Gieseke, M. Gigg, D. Grellscheid, K. Hamilton, et al., Herwig ++ Physics and Manual, Eur. Phys. J. C58 (2008) 639-707, [arXiv:0803.0883].

[101] ATLAS Collaboration, G. Aad et al., The ATLAS Simulation Infrastructure, Eur. Phys. J. C70 (2010) 823-874, [arXiv:1005.4568].

[102] GEANT4 Collaboration, S. Agostinelli et al., GEANT4: A Simulation toolkit, Nucl. Instrum. Meth. A506 (2003) 250-303.

[103] ATLAS Public Results, http://https://twiki.cern.ch/twiki/bin/view/AtlasPublic. Accessed: $22 / 01 / 2014$.

[104] R. E. Kalman, A new approach to linear filtering and prediction problems, Transactions of the ASME-Journal of Basic Engineering 82 (1960), no. Series D 35-45.

[105] ATLAS Collaboration, G. Aad et al., Charged-particle multiplicities in pp interactions measured with the ATLAS detector at the LHC, New J. Phys. 13 (2011) 053033, [arXiv:1012.5104].

[106] ATLAS Collaboration, A measurement of the muon reconstruction efficiency in 2010 ATLAS data using $J / \psi$ decays,

[107] ATLAS Collaboration, Preliminary results on the muon reconstruction efficiency, momentum resolution, and momentum scale in ATLAS $2012 \mathrm{pp}$ collision data, ATLAS-CONF-2013-088.

[108] Y.-S. Tsai, Pair Production and Bremsstrahlung of Charged Leptons, Rev. Mod. Phys. 46 (1974) 815.

[109] ATLAS Collaboration, G. Aad et al., Measurement of the inclusive isolated prompt photon cross section in pp collisions at $\sqrt{s}=7 \mathrm{TeV}$ with the ATLAS detector, Phys. Rev. D83 (2011) 052005, [arXiv: 1012.4389].

[110] ATLAS Collaboration, G. Aad et al., Improved luminosity determination in pp collisions at $\sqrt{s}=7 \mathrm{TeV}$ using the ATLAS detector at the LHC, Eur. Phys. J. C73 (2013) 2518, [arXiv:1302.4393].

[111] S. van der Meer, Calibration of the Effective Beam Height in the ISR, CERN-ISR-PO-68-31.

[112] K. Han et al., Observation of P-wave b $\bar{b}$ bound states, Phys. Rev. Lett. 49 (Nov, 1982) 1612-1616.

[113] G. Eigen et al., Evidence for $\chi_{b}^{\prime}$ production in the exclusive reaction $\Upsilon^{\prime \prime} \rightarrow \chi_{b}^{\prime} \gamma \rightarrow \Upsilon^{\prime} \gamma \gamma$ or $\Upsilon \gamma \gamma$, Phys. Rev. Lett. 49 (Nov, 1982) 1616-1619. 
[114] C. Klopfenstein et al., Observation of the lowest $P$-wave $b \bar{b}$ bound states, Phys. Rev. Lett. 51 (Jul, 1983) 160-163.

[115] F. Daghighian and D. Silverman, Relativistic formulation of the radiative transitions of charmonium and b-quarkonium, Phys. Rev. D 36 (Dec, 1987) 3401-3416.

[116] W. Kwong and J. L. Rosner, D-wave quarkonium levels of the $\Upsilon$ family, Phys. Rev. D 38 (Jul, 1988) 279-297.

[117] L. Motyka and K. Zalewski, Mass spectra and leptonic decay widths of heavy quarkonia, Eur. Phys. J. C4 (1998) 107-114, [hep-ph/9709254].

[118] L. Landau, On the angular momentum of a two-photon system, Dokl. Akad. Nauk Ser. Fiz. 60 (1948) 207-209.

[119] C.-N. Yang, Selection Rules for the Dematerialization of a Particle Into Two Photons, Phys. Rev. 77 (1950) 242-245.

[120] ATLAS Collaboration, Tracking Results and Comparison to Monte Carlo simulation at $\sqrt{s}=900 \mathrm{GeV}$, ATLAS-CONF-2010-011.

[121] ATLAS Collaboration, G. Aad et al., Measurements of Higgs boson production and couplings in diboson final states with the ATLAS detector at the LHC, Phys. Lett. B726 (2013) 88-119, [arXiv:1307.1427].

[122] Belle Collaboration, H. Ikeda et al., A detailed test of the $\mathrm{CsI}(\mathrm{Tl})$ calorimeter for Belle with photon beams of energy between $20 \mathrm{MeV}$ and $5.4 \mathrm{GeV}$, Nucl. Instrum. Meth. A441 (2000) 401-426.

[123] K. S. Cranmer, Kernel estimation in high-energy physics, Comput. Phys. Commun. 136 (2001) 198-207, [hep-ex/0011057].

[124] ATLAS Collaboration, G. Aad et al., Electron performance measurements with the ATLAS detector using the 2010 LHC proton-proton collision data, Eur. Phys. J. C72 (2012) 1909, [arXiv:1110.3174].

[125] CMS Collaboration, Measurement of the $\chi_{b 2}(1 P) / \chi_{b 1}(1 P)$ production cross section ratio in pp collisions at $\sqrt{s}=8 \mathrm{TeV}$, CMS-PAS-BPH-13-005.

[126] G. Cowan, K. Cranmer, E. Gross, and O. Vitells, Asymptotic formulae for likelihood-based tests of new physics, Eur. Phys. J. C71 (2011) 1554, [arXiv: 1007.1727].

[127] Do Collaboration, V. M. Abazov et al., Observation of a narrow mass state decaying into $\Upsilon(1 S)+\gamma$ in pp collisions at $\sqrt{s}=1.96 \mathrm{TeV}$, Phys. Rev. D86 (2012) 031103, [arXiv:1203.6034].

[128] LHCb Collaboration, Observation of the $\chi_{b}(3 P)$ state at LHCb in $p p$ collisions at $\sqrt{s}=7 \mathrm{TeV}$, LHCb-CONF-2012-020. 
[129] CLEO Collaboration, G. Bonvicini et al., First observation of a $\Upsilon(1 D)$ state, Phys. Rev. D70 (2004) 032001, [hep-ex/0404021].

[130] W.-S. Hou, Searching for the bottom counterparts of X(3872) and Y(4260) via $\pi^{+} \pi^{-} \Upsilon$, Phys. Rev. D74 (2006) 017504, [hep-ph/0606016].

[131] D. Ebert, R. Faustov, and V. Galkin, Relativistic model of hidden bottom tetraquarks, Mod. Phys. Lett. A24 (2009) 567-573, [arXiv:0812.3477].

[132] M. T. AlFiky, F. Gabbiani, and A. A. Petrov, X(3872): Hadronic molecules in effective field theory, Phys. Lett. B640 (2006) 238-245, [hep-ph/0506141].

[133] CMS Collaboration, S. Chatrchyan et al., Search for a new bottomonium state decaying to $\Upsilon(1 S) \pi^{+} \pi^{-}$in pp collisions at $\sqrt{s}=8 \mathrm{TeV}$, Phys. Lett. B727 (2013) 57-76, [arXiv: 1309.0250].

[134] S. F. Radford and W. W. Repko, Potential model results for the newly discovered $\chi_{b}(3 P)$ states, arXiv:1201.4096.

[135] C. O. Dib and N. Neill, $\chi_{b}(3 P)$ splitting predictions in potential models, Phys. Rev. D86 (2012) 094011, [arXiv:1208.2186].

[136] W. W. Repko, M. D. Santia, and S. F. Radford, Two-loop Static QCD Potential in Heavy Quarkonia, arXiv:1211.6373.

[137] A. Badalian and B. Bakker, The vector coupling $\alpha_{\mathrm{V}}(r)$ and the scales $r_{0}, r_{1}$ from the bottomonium spectrum, arXiv:1303.2815.

[138] J. Ferretti and E. Santopinto, Higher bottomonia, arXiv:1306.2874.

[139] T. Wei-Zhao, C. Lu, Y. You-Chang, and C. Hong, Bottomonium states versus recent experimental observations in the QCD-inspired potential model, Chin. Phys. C37 (2013) 083101, [arXiv:1308.0960].

[140] ATLAS Collaboration, Measurement of $\chi_{c 1}$ and $\chi_{c 2}$ production with $\sqrt{s}=7 \mathrm{TeV} p p$ collisions at ATLAS, ATLAS-CONF-2013-095.

[141] ATLAS Collaboration, G. Aad et al., Measurement of $\chi_{c 1}$ and $\chi_{c 1}$ production with $\sqrt{s}=7 \mathrm{TeV}$ pp collisions at ATLAS, arXiv:1404.7035.

[142] Belle Collaboration, K. Abe et al., Observation of a near-threshold $\omega J / \psi$ mass enhancement in exclusive $B \rightarrow K \omega J / \psi$ decays, Phys. Rev. Lett. 94 (2005) 182002, [hep-ex/0408126].

[143] BaBar Collaboration, B. Aubert et al., Observation of $Y(3940) \rightarrow J / \psi \omega$ in $B \rightarrow J / \psi \omega K$ at BaBaR, Phys. Rev. Lett. 101 (2008) 082001, [arXiv:0711.2047].

[144] Belle Collaboration, S. Uehara et al., Observation of a $\chi_{c 2}^{\prime}$ candidate in $\gamma \gamma \rightarrow D \bar{D}$ production at Belle, Phys. Rev. Lett. 96 (2006) 082003, [hep-ex/0512035]. 
[145] BaBar Collaboration, B. Aubert et al., Observation of the $\chi_{c 2}(2 P)$ meson in the reaction $\gamma \gamma \rightarrow D \bar{D}$ at BaBaR, Phys. Rev. D81 (2010) 092003, [arXiv: 1002.0281].

[146] E. J. Eichten, K. Lane, and C. Quigg, Charmonium levels near threshold and the narrow state $X(3872) \rightarrow \pi^{+} \pi^{-} J / \psi$, Phys. Rev. D69 (2004) 094019, [hep-ph/0401210].

[147] C. Meng and K.-T. Chao, Decays of the $X(3872)$ and $\chi_{c 1}(2 P)$ charmonium, Phys. Rev. D75 (2007) 114002, [hep-ph/0703205].

[148] CDF Collaboration, T. Affolder et al., Production of $\chi_{c 1}$ and $\chi_{c 2}$ in $p \bar{p}$ collisions at $\sqrt{s}=1.8 \mathrm{TeV}$, Phys.Rev.Lett. 86 (2001) 3963-3968.

[149] LHCb Collaboration, R. Aaij et al., Measurement of the relative rate of prompt $\chi_{c 0}, \chi_{c 1}$ and $\chi_{c 2}$ production at $\sqrt{s}=7 \mathrm{TeV}$, JHEP 1310 (2013) 115, [arXiv:1307.4285].

[150] G. Punzi, Comments on likelihood fits with variable resolution, eConf C030908 (2003) WELT002, [physics/0401045].

[151] M. Oreglia, A Study of the Reactions $\psi^{\prime} \rightarrow \gamma \gamma \psi$. PhD thesis, 1980.

[152] J. Gaiser, Charmonium Spectroscopy From Radiative Decays of the $J / \psi$ and $\psi^{\prime}$. PhD thesis, 1982.

[153] Y.-Q. Ma, K. Wang, and K.-T. Chao, QCD radiative corrections to $\chi_{c j}$ production at hadron colliders, Phys. Rev. D 83 (Jun, 2011) 111503.

[154] H.-S. Shao, HELAC-Onia: an automatic matrix element generator for heavy quarkonium physics, arXiv:1212.5293.

[155] Y.-Q. Ma, K. Wang, and K.-T. Chao, $J / \psi\left(\psi^{\prime}\right)$ production at the Tevatron and $L H C$ at $\mathcal{O}\left(\alpha_{s}^{4} v^{4}\right)$ in nonrelativistic QCD, Phys. Rev. Lett. 106 (2011) 042002, [arXiv: 1009.3655].

[156] L. Harland-Lang and W. A. Stirling, CHIGEN: Monte Carlo Event Generator for inclusive $\chi_{c J}$ production, http://superchic.hepforge.org/chigen.html.

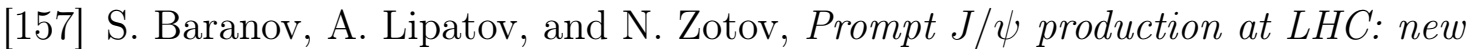
evidence for the $k_{T}$-factorization, Phys. Rev. D85 (2012) 014034, [arXiv: 1108.2856].

[158] S. Baranov, On the $\sigma\left(\chi_{c 2}\right) / \sigma\left(\chi_{c 1}\right)$ ratio in the $k_{T}$-factorization approach, Phys. Rev. D83 (2011) 034035.

[159] ATLAS Collaboration, Cross-Section Measurement of $\psi(2 \mathrm{~S}) \rightarrow J / \psi\left(\rightarrow \mu^{+} \mu^{-}\right) \pi^{+} \pi^{-}$in $7 \mathrm{TeV}$ pp collisions at ATLAS, ATLAS-CONF-2013-094. 
[160] HERA-B Collaboration, I. Abt et al., Production of the Charmonium States $\chi_{c 1}$ and $\chi_{c 2}$ in Proton Nucleus Interactions at $\sqrt{s}=41.6 \mathrm{GeV}$, Phys. Rev. D79 (2009) 012001, [arXiv:0807.2167].

[161] Belle Collaboration, V. Bhardwaj et al., Observation of $X(3872) \rightarrow J / \psi \gamma$ and search for $X(3872) \rightarrow \psi^{\prime} \gamma$ in $B$ decays, Phys. Rev. Lett. 107 (2011) 091803, [arXiv: 1105.0177].

[162] BaBar Collaboration, B. Aubert et al., Evidence for $X(3872) \rightarrow \psi(2 S) \gamma$ in $B^{ \pm} \rightarrow X(3872) K^{ \pm}$decays, and a study of $B \rightarrow c \bar{c} \gamma K$, Phys. Rev. Lett. 102 (2009) 132001, [arXiv:0809.0042].

[163] ATLAS Collaboration, G. Aad et al., Measurement of the differential cross-section of $B^{+}$meson production in pp collisions at $\sqrt{s}=7 \mathrm{TeV}$ at ATLAS, JHEP 1310 (2013) 042, [arXiv:1307.0126].

[164] BaBar Collaboration, B. Aubert et al., Measurement of branching fractions for exclusive $B$ decays to charmonium final states, Phys. Rev. D65 (2002) 032001, [hep-ex/0107025].

[165] CDF Collaboration, D. Acosta et al., Branching ratio measurements of exclusive $B^{+}$decays to charmonium with the Collider Detector at Fermilab, Phys. Rev. D66 (2002) 052005. 
Appendix 


\section{Appendix A}

\section{TABULATED RESULTS}

The following tables show the numerical results presented graphically in Section 7.9.

Statistical and systematic uncertainties are shown for each measurement along with the uncertainty envelope associated with the unknown $\chi_{c}$ polarisation.

\begin{tabular}{|c|c|c|c|c|c|c|}
\hline \hline \multicolumn{2}{|c|}{} & \multicolumn{3}{c|}{$\mathcal{B}\left(\chi_{c J} \rightarrow J / \psi \gamma\right) \cdot \mathcal{B}\left(J / \psi \rightarrow \mu^{+} \mu^{-}\right) \cdot \frac{\mathrm{d} \sigma_{J}^{\mathrm{P}}}{\mathrm{d} p_{T}}[\mathrm{pb} / \mathrm{GeV}]$} \\
\hline$p_{T}^{J / \psi}[\mathrm{GeV}]$ & $\left\langle p_{T}^{J / \psi}\right\rangle[\mathrm{GeV}]$ & $J$ & Value & (stat. $)$ & (syst.) & Polarisation envelope \\
\hline \multirow{2}{*}{$10.0-12.0$} & 11.0 & 1 & 218 & \pm 9 & \pm 28 & $+69-28$ \\
& 11.0 & 2 & 95 & \pm 6 & \pm 12 & $+34-21$ \\
\hline \multirow{2}{*}{$12.0-14.0$} & 12.9 & 1 & 90 & \pm 4 & \pm 11 & $+31-12$ \\
& 12.9 & 2 & 40 & \pm 3 & \pm 5 & $+15-10$ \\
\hline \multirow{2}{*}{$14.0-16.0$} & 14.9 & 1 & 37 & \pm 2 & \pm 5 & $+13-5$ \\
& 14.9 & 2 & 19 & \pm 2 & \pm 2 & $+7-5$ \\
\hline \multirow{2}{*}{$16.0-18.0$} & 16.9 & 1 & 21 & \pm 1 & \pm 3 & $+7-3$ \\
& 16.9 & 2 & 10 & \pm 1 & \pm 1 & $+4-2$ \\
\hline \multirow{2}{*}{$18.0-30.0$} & 22.1 & 1 & 4.8 & \pm 0.2 & \pm 0.6 & $+1.5-0.6$ \\
& 22.1 & 2 & 1.9 & \pm 0.2 & \pm 0.2 & $+0.6-0.4$ \\
\hline
\end{tabular}

Table A.1: Differential cross section for prompt $\chi_{c 1}$ and $\chi_{c 2}$ production, measured in bins of $p_{T}^{J / \psi}$. 


\begin{tabular}{|c|c|c|c|c|c|c|}
\hline \hline \multicolumn{2}{|c|}{} & \multicolumn{5}{|c|}{$\mathcal{B}\left(\chi_{c J} \rightarrow J / \psi \gamma\right) \cdot \mathcal{B}\left(J / \psi \rightarrow \mu^{+} \mu^{-}\right) \cdot \frac{\mathrm{d} \sigma_{J}^{\mathrm{NP}}}{\mathrm{d} p_{T}}[\mathrm{pb} / \mathrm{GeV}]$} \\
\hline$p_{T}^{J / \psi}[\mathrm{GeV}]$ & $\left\langle p_{T}^{J / \psi}\right\rangle[\mathrm{GeV}]$ & $J$ & Value & (stat. $)$ & $($ syst. $)$ & Polarisation envelope \\
\hline \multirow{2}{*}{$10.0-12.0$} & 11.0 & 1 & 60 & \pm 6 & \pm 8 & $+19-8$ \\
& 11.0 & 2 & 15 & \pm 5 & \pm 3 & $+5-3$ \\
\hline \multirow{2}{*}{$12.0-14.0$} & 12.9 & 1 & 30 & \pm 3 & \pm 4 & $+10-4$ \\
& 12.9 & 2 & 4.1 & \pm 2.8 & \pm 0.7 & $+1.6-1.0$ \\
\hline \multirow{2}{*}{$14.0-16.0$} & 14.9 & 1 & 15 & \pm 2 & \pm 2 & $+5-2$ \\
& 14.9 & 2 & 2.9 & \pm 1.1 & \pm 0.5 & $+1.1-0.7$ \\
\hline \multirow{2}{*}{$16.0-18.0$} & 16.9 & 1 & 5.8 & \pm 1.1 & \pm 0.8 & $+1.9-0.7$ \\
& 16.9 & 2 & 0.9 & \pm 0.8 & \pm 0.2 & $+0.3-0.2$ \\
\hline \multirow{2}{*}{$18.0-30.0$} & 22.1 & 1 & 2.1 & \pm 0.3 & \pm 0.3 & $+0.6-0.2$ \\
& 22.1 & 2 & 0.4 & \pm 0.1 & \pm 0.1 & $+0.1-0.1$ \\
\hline \hline
\end{tabular}

Table A.2: Differential cross section for non-prompt $\chi_{c 1}$ and $\chi_{c 2}$ production, measured in bins of $p_{T}^{J / \psi}$.

\begin{tabular}{|c|c|c|c|c|c|c|}
\hline \hline \multicolumn{2}{|c|}{} & \multicolumn{5}{|c|}{$\mathcal{B}\left(\chi_{c J} \rightarrow J / \psi \gamma\right) \cdot \mathcal{B}\left(J / \psi \rightarrow \mu^{+} \mu^{-}\right) \cdot \frac{\mathrm{d} \sigma_{J}^{\mathrm{P}}}{\mathrm{d} p_{T}}[\mathrm{pb} / \mathrm{GeV}]$} \\
\hline$p_{T}^{\chi_{c}}[\mathrm{GeV}]$ & $\left\langle p_{T}^{\chi_{c}}\right\rangle[\mathrm{GeV}]$ & $J$ & Value & $($ stat. $)$ & $($ syst. $)$ & Polarisation envelope \\
\hline \multirow{2}{*}{$12.0-14.0$} & 12.9 & 1 & 136 & \pm 7 & \pm 16 & $+41-17$ \\
& 12.9 & 2 & 73 & \pm 5 & \pm 9 & $+25-15$ \\
\hline \multirow{2}{*}{$14.0-16.0$} & 14.9 & 1 & 71 & \pm 3 & \pm 9 & $+22-8$ \\
& 14.9 & 2 & 29 & \pm 2 & \pm 4 & $+10-6$ \\
\hline \multirow{2}{*}{$16.0-18.0$} & 16.9 & 1 & 31 & \pm 2 & \pm 4 & $+10-4$ \\
& 16.9 & 2 & 18 & \pm 1 & \pm 2 & $+6-4$ \\
\hline \multirow{2}{*}{$18.0-22.0$} & 19.6 & 1 & 15.4 & \pm 0.8 & \pm 1.8 & $+4.4-1.8$ \\
& 19.7 & 2 & 7.0 & \pm 0.6 & \pm 0.9 & $+2.1-1.4$ \\
\hline \multirow{2}{*}{$22.0-30.0$} & 25.0 & 1 & 4.0 & \pm 0.2 & \pm 0.5 & $+1.0-0.4$ \\
& 25.0 & 2 & 1.7 & \pm 0.2 & \pm 0.2 & $+0.4-0.3$ \\
\hline \hline
\end{tabular}

Table A.3: Differential cross section for prompt $\chi_{c 1}$ and $\chi_{c 2}$ production, measured in bins of $p_{T}^{\chi_{c}}$. 


\begin{tabular}{|c|c|c|c|c|c|c|}
\hline \hline \multicolumn{2}{|c|}{} & \multicolumn{5}{|c|}{$\mathcal{B}\left(\chi_{c J} \rightarrow J / \psi \gamma\right) \cdot \mathcal{B}\left(J / \psi \rightarrow \mu^{+} \mu^{-}\right) \cdot \frac{\mathrm{d} \sigma_{J}^{\mathrm{NP}}}{\mathrm{d} p_{T}}[\mathrm{pb} / \mathrm{GeV}]$} \\
\hline$p_{T}^{\chi_{c}}[\mathrm{GeV}]$ & $\left\langle p_{T}^{\chi_{c}}\right\rangle[\mathrm{GeV}]$ & $J$ & Value & $($ stat. $)$ & $($ syst. $)$ & Polarisation envelope \\
\hline \multirow{2}{*}{$12.0-14.0$} & 12.9 & 1 & 42 & \pm 5 & \pm 5 & $+13-5$ \\
& 12.9 & 2 & 9 & \pm 4 & \pm 1 & $+3-2$ \\
\hline \multirow{2}{*}{$14.0-16.0$} & 14.9 & 1 & 23 & \pm 2 & \pm 3 & $+7-3$ \\
& 14.9 & 2 & 2.7 & \pm 1.8 & \pm 0.4 & $+0.9-0.6$ \\
\hline \multirow{2}{*}{$16.0-18.0$} & 16.9 & 1 & 10 & \pm 1 & \pm 1 & $+3-1$ \\
& 16.9 & 2 & 1.8 & \pm 0.8 & \pm 0.3 & $+0.6-0.4$ \\
\hline \multirow{2}{*}{$18.0-22.0$} & 19.6 & 1 & 5.2 & \pm 0.6 & \pm 0.6 & $+1.5-0.6$ \\
& 19.7 & 2 & 0.7 & \pm 0.3 & \pm 0.1 & $+0.2-0.1$ \\
\hline \multirow{2}{*}{$22.0-30.0$} & 25.0 & 1 & 2.1 & \pm 0.3 & \pm 0.3 & $+0.5-0.2$ \\
& 25.0 & 2 & 0.27 & \pm 0.17 & \pm 0.04 & $+0.07-0.05$ \\
\hline \hline
\end{tabular}

Table A.4: Differential cross section for non-prompt $\chi_{c 1}$ and $\chi_{c 2}$ production, measured in bins of $p_{T}^{\chi_{c}}$.

\begin{tabular}{|c|c|c|c|c|}
\hline \hline & \multicolumn{4}{|c|}{ Prompt $R_{\chi_{c}}$} \\
\hline$p_{T}^{J / \psi}[\mathrm{GeV}]$ & Value & (stat.) & (syst.) & Polarisation envelope \\
\hline $10.0-12.0$ & 0.24 & \pm 0.02 & \pm 0.04 & $+0.08-0.04$ \\
\hline $12.0-14.0$ & 0.26 & \pm 0.01 & \pm 0.04 & $+0.09-0.04$ \\
\hline $14.0-16.0$ & 0.23 & \pm 0.02 & \pm 0.04 & $+0.08-0.04$ \\
\hline $16.0-18.0$ & 0.28 & \pm 0.03 & \pm 0.05 & $+0.10-0.05$ \\
\hline $18.0-30.0$ & 0.27 & \pm 0.03 & \pm 0.05 & $+0.09-0.04$ \\
\hline \hline
\end{tabular}

Table A.5: Fraction of prompt $J / \psi$ produced in feed-down from $\chi_{c}$ decays as a function of $p_{T}^{J / \psi}$. The polarisation envelope assumes that prompt $J / \psi$ are produced unpolarised and represents the maximum uncertainty in the result due to the unknown $\chi_{c}$ polarisation.

\begin{tabular}{|c|c|c|c|c|}
\hline & \multicolumn{4}{|c|}{ Prompt $\frac{\sigma\left(\chi_{c 2}\right) \cdot \mathcal{B}\left(\chi_{c 2 \rightarrow J / \psi \gamma}\right)}{\sigma\left(\chi_{c 1}\right) \cdot \mathcal{B}\left(\chi_{c 1 \rightarrow J / \psi \gamma}\right)}$} \\
\hline$p_{T}^{J / \psi}[\mathrm{GeV}]$ & Value & (stat.) & (syst.) & Polarisation envelope \\
\hline $10.0-12.0$ & 0.43 & \pm 0.04 & \pm 0.03 & $+0.24-0.18$ \\
\hline $12.0-14.0$ & 0.44 & \pm 0.04 & \pm 0.03 & $+0.26-0.19$ \\
\hline $14.0-16.0$ & 0.52 & \pm 0.06 & \pm 0.04 & $+0.30-0.23$ \\
\hline $16.0-18.0$ & 0.48 & \pm 0.06 & \pm 0.03 & $+0.27-0.21$ \\
\hline $18.0-30.0$ & 0.40 & \pm 0.04 & \pm 0.03 & $+0.20-0.16$ \\
\hline
\end{tabular}

Table A.6: Production rate of prompt $\chi_{c 2}$ relative to prompt $\chi_{c 1}$, measured in bins of $p_{T}^{J / \psi}$. 


\begin{tabular}{|c|c|c|c|c|}
\hline \hline & \multicolumn{4}{|c|}{ Non-prompt $\left.\frac{\sigma\left(\chi_{c 2}\right) \cdot \mathcal{B}\left(\chi_{c 2} \rightarrow J / \psi \gamma\right.}{\sigma\left(\chi_{c 1}\right) \cdot \mathcal{B}\left(\chi_{c 1} \rightarrow J / \psi \gamma\right.}\right)$} \\
\hline$p_{T}^{J / \psi}[\mathrm{GeV}]$ & Value & (stat.) & (syst.) & Polarisation envelope \\
\hline $10.0-12.0$ & 0.25 & \pm 0.09 & \pm 0.03 & $+0.14-0.10$ \\
\hline $12.0-14.0$ & 0.14 & \pm 0.09 & \pm 0.02 & $+0.08-0.06$ \\
\hline $14.0-16.0$ & 0.19 & \pm 0.08 & \pm 0.02 & $+0.10-0.08$ \\
\hline $16.0-18.0$ & 0.16 & \pm 0.14 & \pm 0.02 & $+0.09-0.07$ \\
\hline $18.0-30.0$ & 0.18 & \pm 0.07 & \pm 0.02 & $+0.09-0.07$ \\
\hline \hline
\end{tabular}

Table A.7: Production rate of non-prompt $\chi_{c 2}$ relative to non-prompt $\chi_{c 1}$, measured in bins of $p_{T}^{J / \psi}$.

\begin{tabular}{|c|c|c|c|c|c|}
\hline \hline & \multicolumn{5}{|c|}{$f_{\text {non-prompt }}$} \\
\hline$p_{T}^{\chi c}[\mathrm{GeV}]$ & $J$ & Value & (stat.) & (syst.) & Polarisation envelope \\
\hline \multirow{2}{*}{$12.0-14.0$} & 1 & 0.23 & \pm 0.02 & \pm 0.02 & $+0.08-0.07$ \\
& 2 & 0.11 & \pm 0.04 & \pm 0.01 & $+0.07-0.04$ \\
\hline \multirow{2}{*}{$14.0-16.0$} & 1 & 0.24 & \pm 0.02 & \pm 0.02 & $+0.08-0.07$ \\
& 2 & 0.09 & \pm 0.05 & \pm 0.01 & $+0.05-0.03$ \\
\hline \multirow{2}{*}{$16.0-18.0$} & 1 & 0.24 & \pm 0.03 & \pm 0.02 & $+0.08-0.06$ \\
& 2 & 0.09 & \pm 0.04 & \pm 0.01 & $+0.05-0.03$ \\
\hline \multirow{2}{*}{$18.0-22.0$} & 1 & 0.25 & \pm 0.03 & \pm 0.02 & $+0.08-0.07$ \\
& 2 & 0.09 & \pm 0.04 & \pm 0.01 & $+0.05-0.03$ \\
\hline \multirow{2}{*}{$22.0-30.0$} & 1 & 0.34 & \pm 0.03 & \pm 0.03 & $+0.08-0.07$ \\
& 2 & 0.14 & \pm 0.07 & \pm 0.02 & $+0.06-0.04$ \\
\hline \hline
\end{tabular}

Table A.8: Fraction of $\chi_{c 1}$ and $\chi_{c 2}$ produced in $b$-hadron decays as a function of $p_{T}^{\chi_{c}}$. 


\begin{tabular}{|c|c|c|c|c|c|c|}
\hline Bin & Yield & Helicity 0 & Helicity \pm 1 & Helicity \pm 2 & $\mathrm{AZ}+$ & AZ- \\
\hline \multirow{4}{*}{$10 \leq p_{T}^{J / \psi}<12 \mathrm{GeV}$} & P1 & 1.32 & 0.89 & - & 0.91 & 0.87 \\
\hline & $\mathrm{P} 2$ & 0.78 & 0.88 & 1.35 & 1.10 & 1.04 \\
\hline & NP1 & 1.31 & 0.89 & - & 0.91 & 0.87 \\
\hline & NP2 & 0.77 & 0.87 & 1.37 & 1.11 & 1.04 \\
\hline \multirow{4}{*}{$12<p_{T}^{J / \psi}<14 \mathrm{GeV}$} & P1 & 1.34 & 0.88 & - & 0.89 & 0.87 \\
\hline & $\mathrm{P} 2$ & 0.76 & 0.87 & 1.38 & 1.10 & 1.06 \\
\hline & NP1 & 1.33 & 0.88 & - & 0.89 & 0.87 \\
\hline & NP2 & 0.76 & 0.87 & 1.38 & 1.10 & 1.06 \\
\hline \multirow{4}{*}{$14<p_{T}^{J / \psi}<16 \mathrm{GeV}$} & $\mathrm{P} 1$ & 1.35 & 0.88 & - & 0.88 & 0.87 \\
\hline & $\mathrm{P} 2$ & 0.76 & 0.87 & 1.38 & 1.09 & 1.07 \\
\hline & NP1 & 1.34 & 0.88 & - & 0.89 & 0.87 \\
\hline & NP2 & 0.76 & 0.87 & 1.38 & 1.09 & 1.07 \\
\hline \multirow{4}{*}{$16<p_{T}^{J / \psi}<18 \mathrm{GeV}$} & $\mathrm{P} 1$ & 1.35 & 0.88 & - & 0.88 & 0.87 \\
\hline & P2 & 0.76 & 0.87 & 1.37 & 1.09 & 1.07 \\
\hline & NP1 & 1.33 & 0.88 & - & 0.88 & 0.87 \\
\hline & NP2 & 0.76 & 0.87 & 1.37 & 1.09 & 1.07 \\
\hline \multirow{4}{*}{$18<p_{T}^{J / \psi}<30 \mathrm{GeV}$} & P1 & 1.32 & 0.88 & - & 0.89 & 0.88 \\
\hline & P2 & 0.78 & 0.88 & 1.33 & 1.08 & 1.07 \\
\hline & NP1 & 1.30 & 0.89 & - & 0.89 & 0.88 \\
\hline & NP2 & 0.78 & 0.88 & 1.33 & 1.07 & 1.06 \\
\hline \multirow{4}{*}{$12 \leq p_{T}^{\chi_{c}}<14 \mathrm{GeV}$} & $\mathrm{P} 1$ & 1.31 & 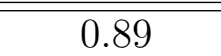 & 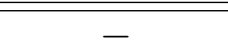 & 0.91 & 0.87 \\
\hline & P2 & 0.78 & 0.88 & 1.35 & 1.10 & 1.04 \\
\hline & NP1 & 1.31 & 0.89 & - & 0.91 & 0.87 \\
\hline & NP2 & 0.78 & 0.88 & 1.35 & 1.10 & 1.04 \\
\hline \multirow{4}{*}{$14<p_{T}^{\chi_{c}}<16 \mathrm{GeV}$} & $\mathrm{P} 1$ & 1.32 & 0.89 & - & 0.90 & 0.88 \\
\hline & $\mathrm{P} 2$ & 0.78 & 0.88 & 1.35 & 1.09 & 1.05 \\
\hline & NP1 & 1.32 & 0.89 & - & 0.90 & 0.88 \\
\hline & NP2 & 0.78 & 0.88 & 1.35 & 1.09 & 1.05 \\
\hline \multirow{4}{*}{$16<p_{T}^{\chi_{c}}<18 \mathrm{GeV}$} & $\mathrm{P} 1$ & 1.32 & 0.89 & - & 0.89 & 0.88 \\
\hline & $\mathrm{P} 2$ & 0.79 & 0.88 & 1.33 & 1.08 & 1.06 \\
\hline & NP1 & 1.32 & 0.89 & - & 0.89 & 0.88 \\
\hline & NP2 & 0.79 & 0.88 & 1.33 & 1.08 & 1.06 \\
\hline \multirow{4}{*}{$18<p_{T}^{\chi_{c}}<22 \mathrm{GeV}$} & P1 & 1.30 & 0.89 & - & 0.90 & 0.89 \\
\hline & P2 & 0.79 & 0.89 & 1.31 & 1.07 & 1.06 \\
\hline & NP1 & 1.30 & 0.89 & - & 0.90 & 0.89 \\
\hline & NP2 & 0.79 & 0.89 & 1.31 & 1.07 & 1.06 \\
\hline \multirow{4}{*}{$22<p_{T}^{\chi_{c}}<30 \mathrm{GeV}$} & $\mathrm{P} 1$ & 1.26 & 0.90 & - & 0.90 & 0.90 \\
\hline & $\mathrm{P} 2$ & 0.81 & 0.90 & 1.27 & 1.06 & 1.05 \\
\hline & NP1 & 1.26 & 0.90 & - & 0.90 & 0.90 \\
\hline & NP2 & 0.81 & 0.90 & 1.27 & 1.06 & 1.05 \\
\hline
\end{tabular}

Table A.9: Scale factors that modify the central cross-section values, evaluated assuming isotropic decay angular distributions, to a given polarisation scenario. The different polarisation scenarios are defined in table 7.2. The labels (N)P1 and (N)P2 correspond to (non-)prompt $\chi_{c 1}$ and (non-)prompt $\chi_{c 2}$ respectively. 


\section{Appendix B}

\section{VALIDATION OF $\chi_{c}$ FITTING PROCEDURE}

The fitting procedure used to extract corrected prompt and non-prompt $\chi_{c 1}$ and $\chi_{c 2}$ yields, described in Section 7.6, is validated using a toy MC simulation technique to ensure the fit results and statistical uncertainties are not strongly biased. Three ensembles of pseudo-data distributions, each containing 500 datasets, are generated from the nominal fit model described in Section 7.6. Each of these three ensembles is designed to imitate the shapes of the $m\left(\mu^{+} \mu^{-} \gamma\right)-m\left(\mu^{+} \mu^{-}\right)$and $\tau$ distributions observed in data for three characteristic transverse momentum bins. The bin denoted "Low" imitates the $\chi_{c J}$ candidates within $10<p_{T}^{J / \psi}<14 \mathrm{GeV}\left(12<p_{T}^{\chi_{c}}<16 \mathrm{GeV}\right)$, "Medium" imitates $14<p_{T}^{J / \psi}<18 \mathrm{GeV}\left(16<p_{T}^{\chi_{c}}<18 \mathrm{GeV}\right)$ and "High" imitates $18<p_{T}^{J / \psi}<30 \mathrm{GeV}\left(18<p_{T}^{\chi_{c}}<30 \mathrm{GeV}\right)$. The pseudo-datasets for each bin are generated with a different background shape, $\tau$ resolution, mixture of prompt and non-prompt $\chi_{c 1}$ and $\chi_{c 2}$ signal and total number of events. All of these characteristics are modelled on the parameters observed in data. Each pseudo-data point is weighted by a single correction weight similar in magnitude to that used in the main analysis (i.e. every candidate is weighted by the same amount). The three ensembles of 500 pseudo-datasets are fitted with the nominal fit model, described in Section 7.6. Example fits to a single pseudo-dataset, for each characteristic $p_{T}$ bin, are shown in Figure B.1.

The pull for each fit to a pseudo-dataset can defined as $\left(N_{\text {truth }}-N_{\text {fit }}\right) / \sigma_{\text {fit }}$, where $N_{\text {truth }}$ is the true number of $\chi_{c}$ candidates generated in the pseudo-dataset, $N_{\text {fit }}$ is 
the number of $\chi_{c}$ candidates determined by the fit and $\sigma_{\text {fit }}$ is the statistical uncertainty on the measured number of candidates. The distribution of pulls for many fits to independent pseudo-datasets can be used to check for biases in the fitting procedure. The distribution of pulls for a perfectly unbiased fit should follow a Gaussian distribution with unit width, centred upon zero. Shifts in the mean of the pull distribution can suggest a bias in the fitting procedure, while deviations from a unit width can suggest that the statistical uncertainty returned by the fit is not reliable. The pull distributions for the fits to the 500 pseudo-datasets generated for each characteristic $p_{T}$ bin are shown in Figure B.2. In general, no consistent systematic bias is observed in the fit results, suggesting that the fitting procedure used to extract the corrected $\chi_{c}$ yields is not strongly biased. The widths of the pull distributions are generally close to unity. With the exception of the non-prompt $\chi_{c 2}$ yields, there is no evidence for a consistent underestimate or overestimate in the statistical uncertainties returned by the fitting procedure. In the case of the nonprompt $\chi_{c 2}$ yields, while no bias in the fit results is observed, the widths of the pull distributions are consistency greater than unity (1.17 on average), suggesting that the statistical uncertainty returned by the fit is slightly underestimated. To correct for this underestimate, the statistical uncertainty in each measured non-prompt $\chi_{c 2}$ yield, when fitting to data, is scaled by 1.17. 

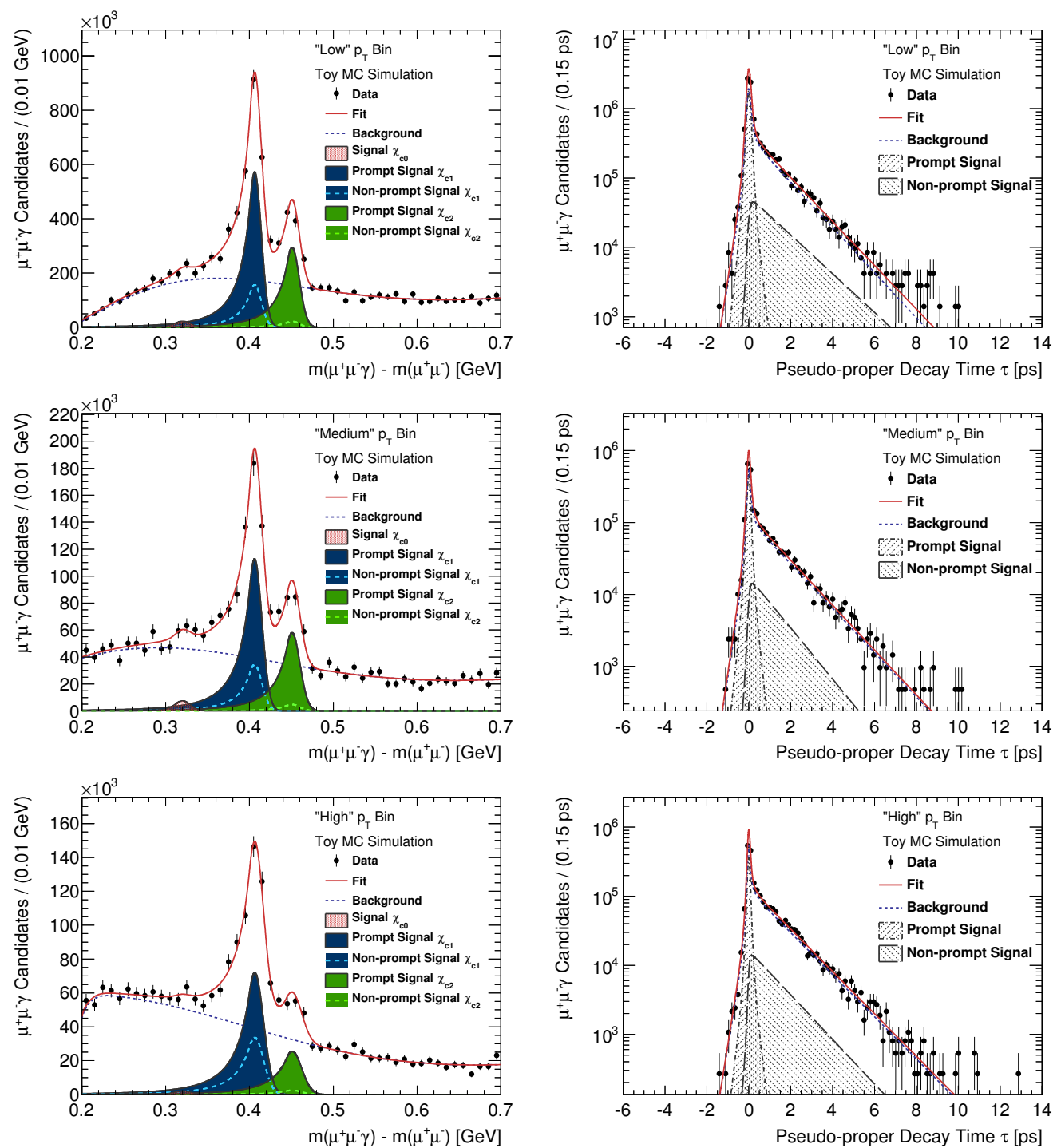

Figure B.1: Simultaneous fits to $m\left(\mu^{+} \mu^{-} \gamma\right)-m\left(\mu^{+} \mu^{-}\right)$and $\tau$ distributions generated using the toy MC method. Pseudo-data are generated in three characteristic $p_{T}$ bins; "Low" $p_{T}$ (top), "Medium" $p_{T}$ (middle) and "High" $p_{T}$ (bottom). 

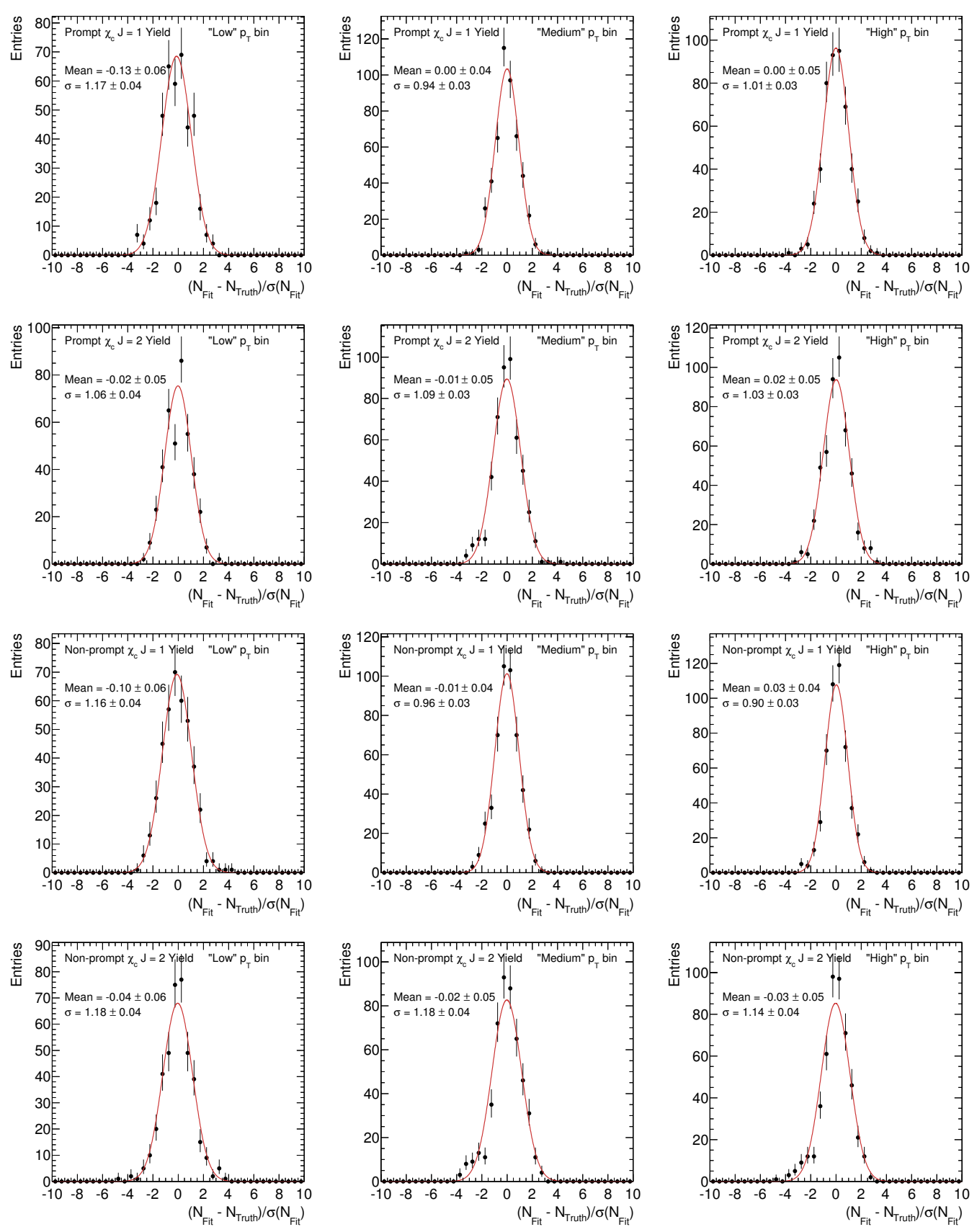

Figure B.2: Pull distributions for prompt and non-prompt $\chi_{c 1}$ and $\chi_{c 2}$ yields in three characteristic $p_{T}$ bins. 